\title{
Molecular imaging of biologic characteristics and drug uptake : towards personalized medicine using dose painting
}

Citation for published version (APA):

Aerts, H. J. W. L. (2010). Molecular imaging of biologic characteristics and drug uptake : towards personalized medicine using dose painting. [Doctoral Thesis, Maastricht University]. Datawyse / Universitaire Pers Maastricht. https://doi.org/10.26481/dis.20101216ha

Document status and date:

Published: 01/01/2010

DOI:

10.26481/dis.20101216ha

Document Version:

Publisher's PDF, also known as Version of record

Please check the document version of this publication:

- A submitted manuscript is the version of the article upon submission and before peer-review. There can be important differences between the submitted version and the official published version of record.

People interested in the research are advised to contact the author for the final version of the publication, or visit the DOI to the publisher's website.

- The final author version and the galley proof are versions of the publication after peer review.

- The final published version features the final layout of the paper including the volume, issue and page numbers.

Link to publication

\footnotetext{
General rights rights.

- You may freely distribute the URL identifying the publication in the public portal. please follow below link for the End User Agreement:

www.umlib.nl/taverne-license

Take down policy

If you believe that this document breaches copyright please contact us at:

repository@maastrichtuniversity.nl

providing details and we will investigate your claim.
}

Copyright and moral rights for the publications made accessible in the public portal are retained by the authors and/or other copyright owners and it is a condition of accessing publications that users recognise and abide by the legal requirements associated with these

- Users may download and print one copy of any publication from the public portal for the purpose of private study or research.

- You may not further distribute the material or use it for any profit-making activity or commercial gain

If the publication is distributed under the terms of Article 25fa of the Dutch Copyright Act, indicated by the "Taverne" license above, 


\section{Molecular imaging of biologic characteristics and drug uptake}

Towards personalized medicine using dose painting

Hugo Aerts 
Cover:

Biography in an abstract form

designed by Hugo Aerts and John Aerts

Druk: Datawyse | Universitaire Pers Maastricht

(C) Copyright H.J.W.L. Aerts, Maastricht 2010

ISBN 9789461590220 


\section{Molecular imaging of biologic characteristics and drug uptake}

Towards personalized medicine using dose painting

\section{PROEFSCHRIFT}

ter verkrijging van de graad van doctor aan de Universiteit Maastricht, op gezag van de Rector Magnificus Prof. mr. G.P.M.F. Mols, volgens het besluit van het College van Decanen, in het openbaar te verdedigen, op donderdag 16 december 2010 om 14.00 uur

door

Hugo Johannes Wilhelmus Louis Aerts

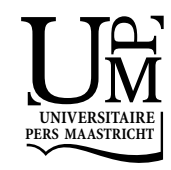




\section{Promotores}

Prof. dr. Ph. Lambin

Prof. dr. D.K.M. De Ruysscher

\section{Copromotor}

Dr. ir. A.L.A.J. Dekker

\section{Beoordelingscommissie}

Prof. dr. J. Wildberger

(Maastricht University, voorzitter)

Prof. dr. R. Gillies

(University of South Florida)

Prof. dr. ir. B. ter Haar Romeny

(Technische Universiteit Eindhoven)

Prof. dr. ir. F. Verhaegen

(Maastricht University)

The work presented in this thesis is made possible by the financial support of: Dutch Cancer Society (KWF), ZonMW, Siemens and the Euroxy 6th framework Grant. 


\section{CONTENTS}

\section{Introduction}

Chapter 1 General introduction and outline of the thesis

Part 1 Identification of radioresistance: Rational for dose painting

Chapter 2 Identification of radioresistance: Identification of residual meta- 23 bolic-active areas using a pre-radiotherapy FDG-PET-CT scan

Chapter 3 Identification of radioresistance: Validation of location of residual $\quad 41$ metabolic-active areas in a Toronto dataset

Chapter 4 Identification of radioresistance: Prospective validation of loca55 tion of residual metabolic-active areas in a Nijmegen dataset

Chapter 5 Stability of FDG uptake locations during treatment

Part 2 Non invasive imaging of Drug uptake: From the bench to the clinic

Chapter 6 Imaging of drug uptake: In vitro imaging of Cetuximab

Chapter 7 Imaging of drug uptake: Pre-clinical in vivo imaging of Cetuximab

Chapter 8 Imaging of drug uptake: Clinical in vivo imaging of Cetuximab

Summary

Samenvatting

Dankwoord

Curriculum Vitae 



\section{PART 1}

Identification of radioresistance:

Rational for Dose Painting 



\section{CHAPTER}

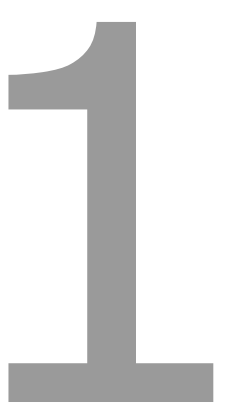

Introduction and outline of the thesis 



\section{INTRODUCTION}

Over the last century, cancer has increasingly become one of the most important causes of death and due to the aging patient population in the developed countries, cancer will remain one of the major health problems of the future ${ }^{1}$. The signing of the National Cancer Act of 1971 by then U.S. President Richard Nixon is in general considered the start of a worldwide "War on Cancer", to eradicate the disease as a major cause of death ${ }^{2,3}$. Although it was not described as a "war" in the legislation itself, the aim of the "War on Cancer" refers to a joint effort to find a cure for cancer by a huge increase of scientific research worldwide, to improve the understanding of cancer biology, and the development of more effective cancer treatments ${ }^{3}$. Hitherto, it was thought that the finding of a cure for cancer was only years away. However, despite enormous amounts of money and time spend in scientific research the last decades, nearly 40 years after this war began, cancer still remains one of the leading causes of death in Europe, the United States and other developed countries. In 2007 cancer accounted for 7.9 million deaths worldwide and around 1.7 million deaths in Europe alone ${ }^{1}$. Figure 1 shows the progression of leading causes of death over time. In contrast to heart disease, stroke, and unintentional injuries, it is apparent that there is little to no reduction in the number of cancer related deaths over the last decades. For example, the prognosis of lung cancer, which causes the most cancer related deaths in men and is the third cause of cancer deaths in women, remains disappointingly poor. Lung cancer can be divided in two subtypes, small cell lung cancer and non-small cell lung cancer (NSCLC), which accounts for the vast majority of all lung cancer cases $( \pm 80 \%)$. Currently, patients with NSCLC have an average one year survival of about $40 \%$ and a 5 -year survival of $15 \%{ }^{1}$. Surgery is often chosen for the treatment of early stage NSCLC patients. Unfortunately, NSCLC is not frequently detected at early stages and typically patients have an advanced stage at the time of diagnosis and are considered inoperable. For these patients, a combination of chemotherapy and radiotherapy is often elected as the treatment modality ${ }^{4}$. Besides distant metastasis, one of the main reasons of poor patient prognosis is that local tumour control commonly fails (e.g. the tumour of $70 \%$ of all NSCLC cancer patients cannot be controlled by cancer treatments) ${ }^{5-7}$. Therefore, new investigations to improve local tumour control are warranted to improve patient outcomes.

\section{Personalized medicine}

Despite little progress in the treatment of cancer over the past century, there has been a tremendous increase in our basic understanding of cancer at the molecular level that has revealed an enormously complex disease. It has become increasingly clear that due to patient specific gene mutations and other specific tumour characteristics, in principle every patient has his own 'disease', and therefore should receive a specific treat- 


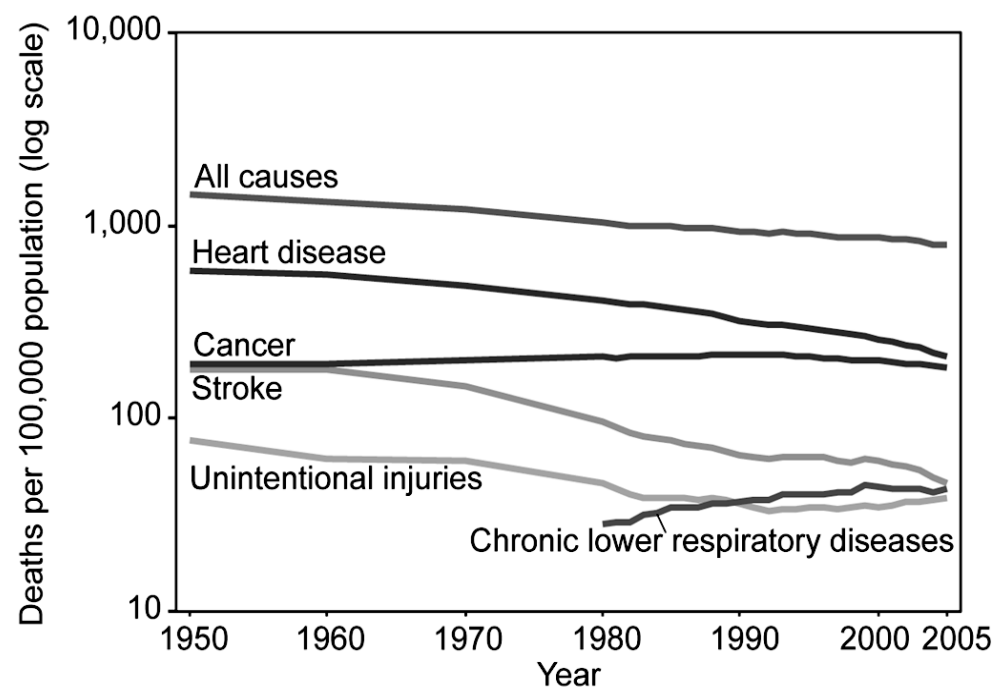

\section{Figure 1}

Leading causes of death for all ages. All death rates are age adjusted.

(Report: Health, United States, 2008National Center for Health Statistics 8)

ment. Besides the standard cancer treatment modalities, like surgery, chemotherapy, and radiotherapy, more and more targeted agents become available to which often only a small subpopulation responds. New medical diagnostic technology has resulted in an ever increasing amount of patient specific information for example, from non-invasive imaging, gene-arrays, pathology, and blood biomarkers. Therefore, it is a challenge to select from all the therapeutic options the optimal treatment based on all the available diagnostic data. The concept of using advanced diagnostic capabilities to tailor treatment is called "personalized medicine". The goal of personalized medicine is to individualize towards the specific characteristics of a patient, tumour genotype and the patient's wishes, instead of the currently common "one size fits all" treatment modalities. This concept is motivated by the fact that patients with similar disease stage often show a large variation in response to systemic therapy and radiotherapy, for both efficacy and toxicity (Figure 2).

\section{The concept of dose painting of radiation}

Radiotherapy is the medical use of high energy photon beams to deliver and localize amounts of ionizing radiation (radiation dose) to regions of malignant cells in thetreatment of cancer. As radiotherapy is the only treatment modality that can exploit its therapeutic effect in the three dimensions of space, dose painting refers to a methodology to individualize the treatment by guiding the radiotherapy dose towards 


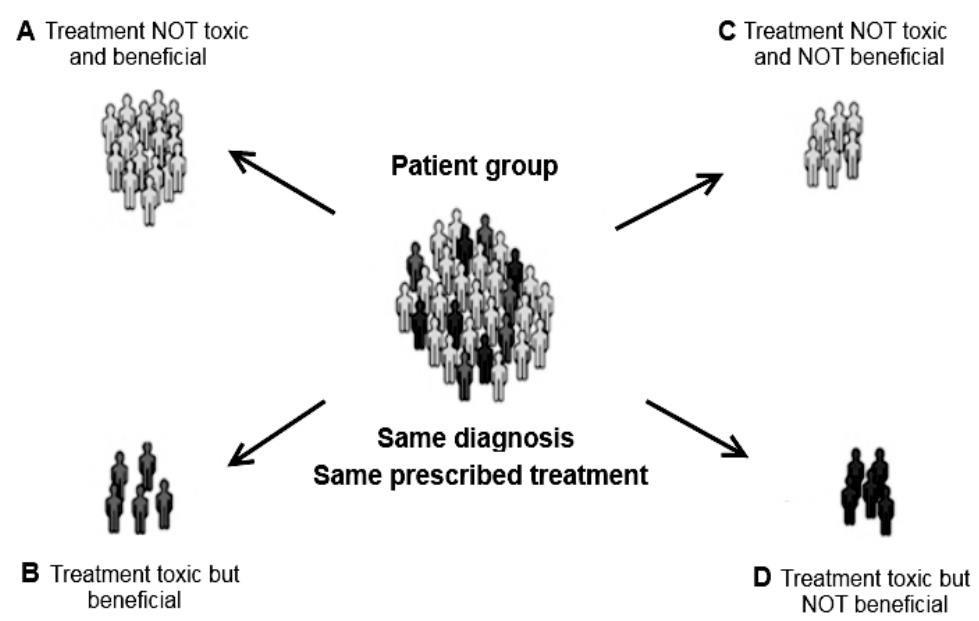

\section{Figure 2}

In a group of patients with the same diagnosis and the same prescribed treatment, there are subpopulations that differ in the benefit and the toxicity of the selected treatment. Note, that only group $A$ has an optimal treatment, group $B$ experiences toxicity, and in group $C$ and $D$ the treatment is even not beneficial.

intra tumour regions of biologic treatment-resistance ${ }^{9-13}$. Indeed, in recent years it has become clear that tumours are not homogeneous,guiding the radiotherapy dose towards intra tumour regions of biologic treatment-resistance ${ }^{9-13}$. Indeed, in recent years it has become clear that tumours are not homogeneous, but heterogeneous for various biologic characteristics, like tumour cell density, normal tissue involvement, vasculature, hypoxia, proliferation, gene expression and drug uptake ${ }^{11,14,15}$. Tumour heterogeneity is exhibited both at the microscopic and macroscopic level. Therefore, it is likely that there exist large spatial differences in the probability to eradicate distinguishable tumour sub-volumes by radiotherapy, chemotherapy, targeted agents, and/or their combinations. Therefore, to strive for a homogeneous radiation dose distribution, as now is current practice, seems illogical as some areas receive a dose that is not enough for eradication, and in other, more sensitive regions, dose is wasted. Also, an overall increase of the dose in the tumour is not possible due to the increasing dose in the normal tissues, resulting in more toxicity after therapy. Therefore, a heterogeneous dose distribution, matching the profile of intra tumour differences of radio-curability, seems to be the rational choice. 


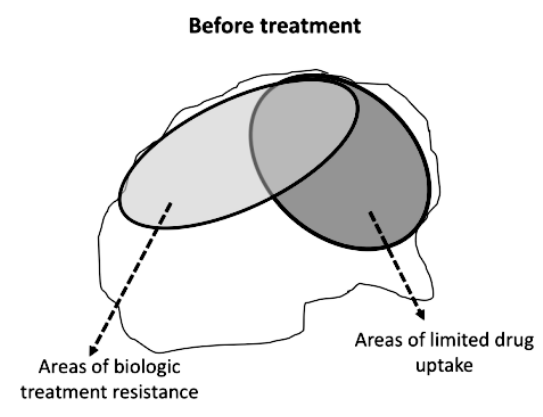

Radiotherapy Dose plan:

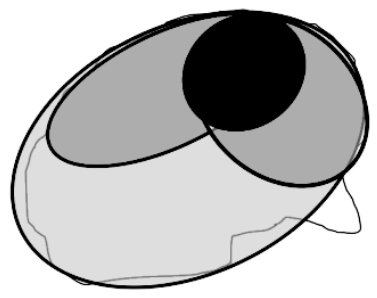

During treatment

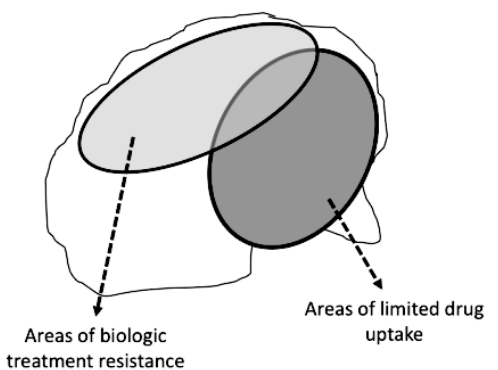

Adapted Radiotherapy Dose plan:

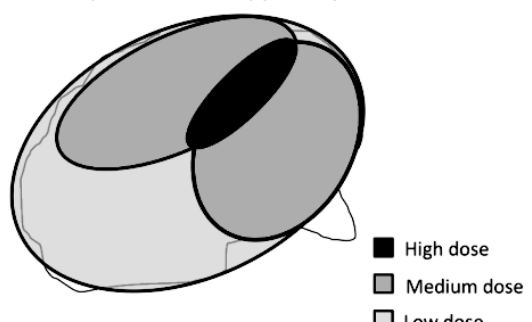

Figure 3

Schematic representation of dose painting exploiting intra tumour heterogeneity of treatment resistance and decreased drug uptake before treatment (left) and during treatment (right). Note that if changes occur during treatment of the intra tumour drug uptake, e.g. due to regions of enhanced perfusion due to RT (top right), the treatment plan has to be adapted (bottom right).

\section{Dose painting in combination with systemic therapies}

For the treatment of the most advanced cancers radiotherapy is often combined with systemic therapies, typically consisting of chemotherapy, immunotherapy or biological response modifiers. These systemic therapies can have large intra tumour differences of the therapeutic effect, due to accessibly issues, e.g. large antibodies have limited reach into the less perfused tumour regions, and due to sensitivity issues of the tumour cells to the drug, e.g. resistance to targeted agents. Therefore, if the areas of failure of systemic therapies can be identified using non-invasive imaging, the dose can be directed towards these 'volumes' of resistance (Figure 3). Here molecular imaging can be used to: I) visualize biologic characteristics known to be related with treatmentresistance, like metabolism and hypoxia, and II) to image the uptake of drugs. Recent advances of PET imaging allow for the visualisation of drug uptake even days after administration with a high sensitivity. As various studies have shown that the absorption of the drugs is often heterogeneous, this indicates that accessibility of tumour tissue by large monoclonal antibodies can be an important factor for intra tumour differences of therapy response and resistance. Therefore, also imaging of drug uptake can be valid to identify tumour sub-volumes for dose painting. 


\section{Identification of biologic characteristics using ${ }^{18} \mathrm{~F}$-deoxyglucose}

Before the concept of dose painting can be tested in clinical settings, it is crucial to identify the regions of resistance of treatment using non-invasive imaging. Here molecular imaging can be used to visualize biologic characteristics known to be related with treatment-resistance, like hypoxia and metabolism. For this purpose novel tracers for positron emission tomography (PET) imaging, like hypoxia markers ${ }^{10,15,16}$, or conventional tracers like ${ }^{18} \mathrm{~F}$-deoxyglucose (FDG) measuring tumour glucose metabolism 17,18 , can be used. FDG is the workhorse in the field of oncology for diagnostic and prognostic purposes that has led to improved clinical decision making in a large quantity of cancer patients ${ }^{19}$. Many investigations have shown that high FDG uptake is a prognostic indicator for poor patient survival both treated with radiotherapy and surgery ${ }^{20-24}$. During the past decades FDG has become widely available and a lot of progress was made in standardisation and reproducibility of FDG-PET imaging ${ }^{25,26}$, further improving the usability in the clinic. The underlying biology for FDG uptake is complex as it measures the uptake of glucose in cells by adenosine triphosphate (ATP) independent glucose transporters. This makes FDG uptake specific to the metabolic pathway although this pathway is known to influence numerous other processes to facilitate cancer ${ }^{27,28}$. Consequently, FDG uptake in the tumour does not reflect a single biologic characteristic, but is correlated to although certainly not specific for, radioresistance, proliferation ${ }^{29}$, cell density ${ }^{30}$, hypoxia ${ }^{24,31-33}$, mitochondrial dysfunction ${ }^{34}$, and lipogenesis ${ }^{35}$. Furthermore, in a pre-clinical investigation, it has been shown that an increase of radiation dose showed a higher local control for tumours with higher FDG uptake compared to tumours with lower FDG uptake ${ }^{36}$, affirming the relation between FDG-uptake and radio-resistance.

A straightforward way to identify recurring regions of treatment resistance is to identify the residual tumour locations following treatment. This approach is referred to as "pattern of relapse studies" in journal publications. One method of investigating the location of the residual tumour masses is assessing metabolic response within the primary tumour with using FDG-PET imaging after treatment ${ }^{17}$. Several studies indicated that patients with metabolically active residual masses after treatment have a poorer prognosis compared to patients without residual metabolic activity ${ }^{17,37}$, underlining its clinical validity.

\section{Non invasive imaging of the drug Cetuximab}

Recent advances in PET imaging allow for the visualisation of drug uptake even days after administration with a high sensitivity. The uptake of drugs can be assessed by labelling the drug itself with radio-nuclides, and follow the tracer uptake over time with PET imaging ${ }^{38}$. An example of such a drug is the monoclonal antibody Cetuximab (IMC-225, Erbitux) specifically targeting the epidermal growth factor receptor (EGFR), a 
molecule at the cell membrane ${ }^{39-41}$. EGFR is a member of the ErbB family and has been shown to play an essential role in the upregulation of tumour cell proliferation, differentiation and survival ${ }^{42,43}$. EGFR expression is commonly found in a significant number of human malignancies and its expression is associated with tumour aggressiveness and overall treatment resistance ${ }^{44,45}$. As a result, EGFR targeting has been explored as a possible anti-tumour strategy. Cetuximab is an example of a monoclonal antibody which blocks the ligand-binding domain of EGFR with high affinity, thereby preventing downstream signalling ${ }^{39,46}$. Consequently, Cetuximab is considered as a promising new targeted agent and is increasingly used in clinical trials, mainly in combination with chemo- or radiotherapy ${ }^{40,47}$. Despite extensive use of Cetuximab in clinical settings, much is unknown about the patient-specific tumour uptake and overall pharmacokinetics of Cetuximab ${ }^{40,44,45}$. Moreover, only a small sub-population of patients benefits from Cetuximab treatment, were often discrepancies between Cetuximab uptake and EGFR expression levels have been reported ${ }^{48-51}$. Also, due to the toxicity related with Cetuximab treatment, there is a strong incentive to identify patients who are less likely to benefit from Cetuximab treatment and spare them undue toxicity. Therefore, the non-invasive quantification of in vivo Cetuximab uptake in tumours and healthy tissue could provide crucial prognostic indicators, usable for selecting patients suitable for Cetuximab treatment and optimizing dosage, but also identifying intra tumour areas for dose painting.

Several groups have used Cetuximab itself as an imaging agent because it has several advantages for visualizing its distribution in normal and tumour tissues. The majority of these Cetuximab-based non-invasiveimaging probes are labelled with SPECT and PET isotopes ${ }^{52-58}$. Some studies were limited by the use of relatively short half-life $\left(t_{1 / 2}\right)$ isotopes ( $\left({ }^{99 \mathrm{~m}} \mathrm{Tc}: 6 \mathrm{~h}\right.$ and ${ }^{64} \mathrm{Cu}: 12 \mathrm{~h}$ ) which are incompatible with the long biologic halflife of Cetuximab in the blood pool ${ }^{55}$. Another isotope for PET imaging often suggested for antibody imaging is lodine-124, because its long physical half-life $\left(t_{1 / 2}=100.3\right.$ hours) allows imaging even days after injection. However, an important disadvantage of lodine-124 is the rapid degradation of the radioiodinated antibody upon internalization, leading to PET images of less quality which does not reflect the actual tumour uptake of the antibody ${ }^{53}$. Based on the findings of Verel et al. ${ }^{38,59}$, the positron emitter Zirconium-89 $\left({ }^{89} \mathrm{Zr}\right)$ displays ideal characteristics for usage with antibodies ${ }^{60} .{ }^{89} \mathrm{Zr}$ demonstrates a long physical half-life ( $t_{1 / 2}=78$ hour) and, upon internalization of the antibody, is trapped intracellularly in lysosomes (phenomenon of residualization) ${ }^{41,61}$. Moreover, radio-labelling of Cetuximab with ${ }^{89} \mathrm{Zr}$ does not influence its binding potential to EGFR, resulting in an immunoreactive fraction of about $95 \%{ }^{60}$. For these reasons, ${ }^{89}$ Zris an ideal radionuclide for the labelling of slow kinetic intact antibodies with high quality standards, and is successfully used in pre-clinical ${ }^{62}$ and clinical settings $53,63,64$ 
It is expected that the implementation of dose painting in combination with systemic therapies in clinical settings, will yield major steps in better local control rates, thereby improving survival and the quality of life, urgently needed in cancer treatment. A prerequisite for the concept of dose painting is that the areas of biologic treatment resistance and limited drug uptake can be identified, as these areas can be compensated with a higher radiation dose.

\section{OBJECTIVE OF THE THESIS}

The general hypothesis is that solid tumours are heterogeneous in their sensitivity to treatment by biologic resistance and drug uptake. Therefore, this thesis investigates if molecular imaging of biologic characteristics and drug uptake can be used to identify regions of treatment resistance, thereby enabling personalized medicine by dose painting. There were two specific research aims:

1) The non-invasive identification treatment-resistant areas using a pre-treatment FDG-PET-CT scan for dose painting studies.

II) The development and validation of novel image probes for pre-clinical and clinical imaging of the drug Cetuximab.

\section{Outline of the thesis}

As the work presented in this thesis can be divided in two distinct parts, these are separately presented to the reader. At first, in this Chapter 1, an introduction for the work presented in this thesis is given. Also the role of radiation oncology in personalize medicine is introduced.

\section{Part 1: Identification of radioresistance: Rational for dose painting}

The first part of this thesis describes the identification of radioresistance using FDGPET-CT imaging. For this purpose critical questions have to be answered: I) is it possible to identify treatment-resistant areas within the tumour using a pre-treatment FDGPET-CT scan? and II) do these areas remain stable within the tumour during a course of radiotherapy?

Chapter 2 presents a study that assessed if it is possible to identify the location of radioresistance using a FDG-PET-CT scan before treatment. Specifically, we assessed for the first time if the location of residual disease after treatment was similar to the location of high FDG-uptake within the tumour before treatment. This work was performed in a MAASTRO dataset. 
In Chapter 3 a study is presented if the results of Chapter 4 can be validated in an independent dataset from the Princess Margaret Hospital (PMH) in Toronto, Canada. Validation in external datasets is important for evidence of the results.

Chapter 4 describes a validation study in another dataset from the Radboud University Medical Center in Nijmegen, the Netherlands. This dataset is not only independent, but also prospectively gathered.

Chapter 5 describesa study that assessed if these FDG uptake zones remained stable during a course of fractionated radiotherapy. This critical question has to be answered before selectively boosting parts of the tumour is possible.

\section{Part 2: Non invasive imaging of drug uptake: From the bench to the clinic}

This second part of the thesis is about the development of image tracers for the visualisation of drug uptake. In specific, we labelled the monoclonal antibody Cetuximab targeting the epidermal growth factor receptor (EGFR). We have developed in vitro and in vivo image probes to investigate the distribution of Cetuximab non-invasively.

In Chapter 6 a study with the fluorescent image probe Cetuximab labelled Oregon Green 488 is presented to the reader. Here, the in vitro binding of Cetuximab to EGFR and the mutant EGFRvIII was investigated, as well as the ex vivo distribution of Cetuximab and the EGFR.

Chapter 7 presents an in vivo study with Cetuximab labelled with the positron emitter Zirconium-89 ( $\left({ }^{89} \mathrm{Zr}\right)$ for PET imaging. Here, the uptake of ${ }^{89} \mathrm{Zr}$-Cetuximab was investigated non-invasively in tumours with varying EGFR expression levels up to days after injection.

In Chapter 8 a study is described that investigated the in vivo uptake of ${ }^{89} \mathrm{Zr}$-Cetuximab in cancer patients. This phase I study was performed to assess the toxicity and image quality in a limited number of patients.

\section{General discussion and future perspectives}

Chapter 9 gives a general discussion of the results presented in this thesis and looks at future perspectives. 


\section{REFERENCES}

1. World Health Organization: Cancer - Key Facts 2007. http://www.who.int/cancer.

2. National Cancer Act of $1971 \mathrm{NCl}$, http://www.cancer.gov/aboutnci/national-cancer-act1971/allpages.

3. Wikipedia, http://en.wikipedia.org/wiki/War_on_cancer.

4. Auperin A, Le Pechoux C, Rolland E, et al., Meta-analysis of concomitant versus sequential radiochemotherapy in locally advanced non-small-cell lung cancer, J Clin Oncol 28 (13), 2181-2190.

5. Zatloukal P, Petruzelka L, Zemanova $M$, et al., Concurrent versus sequential chemoradiotherapy with cisplatin and vinorelbine in locally advanced non-small cell lung cancer: a randomized study, Lung Cancer 46 (1), 87-98 (2004).

6. Furuse K, Fukuoka M, Kawahara M, et al., Phase III study of concurrent versus sequential thoracic radiotherapy in combination with mitomycin, vindesine, and cisplatin in unresectable stage III non-small-cell lung cancer, J Clin Oncol 17 (9), 2692-2699 (1999).

7. Fournel $\mathrm{P}$, Robinet $\mathrm{G}$, Thomas $\mathrm{P}$, et al., Randomized phase III trial of sequential chemoradiotherapy compared with concurrent chemoradiotherapy in locally advanced non-small-cell lung cancer: Groupe Lyon-Saint-Etienne d'Oncologie Thoracique-Groupe Francais de Pneumo-Cancerologie NPC 95-01 Study, J Clin Oncol 23 (25), 5910-5917 (2005).

8. Health, United States (2008), National Center for Health Statistics, http://www.ncbi.nlm.nih.gov/bookshelf/br.fcgi?book=healthus08.

9. Tanderup K, Olsen DR and Grau C, Dose painting: art or science?,Radiother Oncol 79 (3), 245-248 (2006).

10. Thorwarth D, Eschmann SM, Paulsen F, et al., Hypoxia dose painting by numbers: a planning study, Int J Radiat Oncol Biol Phys 68 (1), 291-300 (2007).

11. Bentzen SM, Theragnostic imaging for radiation oncology: dose-painting by numbers, Lancet Oncol 6 (2), 112-117 (2005).

12. Bentzen SM, Dose painting and theragnostic imaging: towards the prescription, planning and delivery of biologically targeted dose distributions in external beam radiation oncology, Cancer Treat Res 139, 41-62 (2008).

13. Ling CC, Humm J, Larson S, et al., Towards multidimensional radiotherapy (MD-CRT): biological imaging and biological conformality, Int J Radiat Oncol Biol Phys 47 (3), 551-560 (2000).

14. Vanderstraeten B, Duthoy W, De Gersem W, et al., [18F]fluoro-deoxy-glucose positron emission tomography ([18F]FDG-PET) voxel intensity-based intensity-modulated radiation therapy (IMRT) for head and neck cancer, Radiother Oncol 79 (3), 249-258 (2006).

15. Foo SS, Abbott DF, Lawrentschuk N, et al., Functional imaging of intratumoral hypoxia, Mol Imaging Biol 6 (5), 291-305 (2004).

16. Krohn KA, Link JM and Mason RP, Molecular imaging of hypoxia, J Nucl Med 49 Suppl 2, 129S-148S (2008).

17. Mac Manus MP, Hicks RJ, Matthews JP, et al., Metabolic (FDG-PET) response after radical radiotherapy/chemoradiotherapy for non-small cell lung cancer correlates with patterns of failure, Lung Cancer 49 (1), 95-108 (2005).

18. Phelps ME, Inaugural article: positron emission tomography provides molecular imaging of biological processes, Proc Natl Acad Sci U S A 97 (16), 9226-9233 (2000). 
19. Jadvar H, Alavi A and Gambhir SS, 18F-FDG uptake in lung, breast, and colon cancers: molecular biology correlates and disease characterization, J Nucl Med 50 (11), 1820-1827 (2009).

20. Vansteenkiste JF, Stroobants SG, Dupont PJ, et al., Prognostic importance of the standardized uptake value on (18)F-fluoro-2-deoxy-glucose-positron emission tomography scan in non-small-cell lung cancer: An analysis of 125 cases. Leuven Lung Cancer Group, J Clin Oncol 17 (10), 3201-3206 (1999).

21. Eschmann SM, Friedel G, Paulsen F, et al., Is standardised (18)F-FDG uptake value an outcome predictor in patients with stage III non-small cell lung cancer?, Eur J Nucl Med Mol Imaging 33 (3), 263-269 (2006).

22. Borst GR, Belderbos JS, Boellaard R, et al., Standardised FDG uptake: a prognostic factor for inoperable non-small cell lung cancer, Eur J Cancer 41 (11), 1533-1541 (2005).

23. Downey RJ, Akhurst T, Gonen M, et al., Preoperative F-18 fluorodeoxyglucose-positron emission tomography maximal standardized uptake value predicts survival after lung cancer resection, J Clin Oncol 22 (16), 3255-3260 (2004).

24. van Baardwijk A, Dooms C, van Suylen RJ, et al., The maximum uptake of (18)Fdeoxyglucose on positron emission tomography scan correlates with survival, hypoxia inducible factor-1alpha and GLUT-1 in non-small cell lung cancer, Eur J Cancer 43 (9), 13921398 (2007).

25. Boellaard R, Standards for PET image acquisition and quantitative data analysis, J Nucl Med 50 Suppl 1, 11S-20S (2009).

26. Boellaard R, Oyen WJ, Hoekstra CJ, et al., The Netherlands protocol for standardisation and quantification of FDG whole body PET studies in multi-centre trials, Eur J Nucl Med Mol Imaging 35 (12), 2320-2333 (2008).

27. Kim JW and Dang CV, Cancer's molecular sweet tooth and the Warburg effect, Cancer Res 66 (18), 8927-8930 (2006).

28. Feron 0 , Pyruvate into lactate and back: from the Warburg effect to symbiotic energy fuel exchange in cancer cells, Radiother Oncol 92 (3), 329-333 (2009).

29. Vesselle $H$, Schmidt RA, Pugsley JM, et al., Lung cancer proliferation correlates with [F18]fluorodeoxyglucose uptake by positron emission tomography, Clin Cancer Res 6 (10), 3837-3844 (2000).

30. Dooms C, van Baardwijk A, Verbeken E, et al., Association between 18F-fluoro-2-deoxy-Dglucose uptake values and tumor vitality: prognostic value of positron emission tomography in early-stage non-small cell lung cancer, J Thorac Oncol 4 (7), 822-828 (2009).

31. Airley R, Loncaster J, Davidson S, et al., Glucose transporter glut-1 expression correlates with tumor hypoxia and predicts metastasis-free survival in advanced carcinoma of the cervix, Clin Cancer Res 7 (4), 928-934 (2001).

32. Sattler UG and Mueller-Klieser W, The anti-oxidant capacity of tumour glycolysis, Int J Radiat Biol 85 (11), 963-971 (2009).

33. Christian N, Deheneffe $\mathrm{S}$, Bol A, et al., Is (18)F-FDG a surrogate tracer to measure tumor hypoxia? Comparison with the hypoxic tracer (14)C-EF3 in animal tumor models, Radiother Oncol.

34. Shanmugam M, McBrayer SK and Rosen ST, Targeting the Warburg effect in hematological malignancies: from PET to therapy, Curr Opin Oncol 21 (6), 531-536 (2009). 
35. Menendez JA and Lupu R, Fatty acid synthase and the lipogenic phenotype in cancer pathogenesis, Nat Rev Cancer 7 (10), 763-777 (2007).

36. Schutze C, Bergmann R, Yaromina A, et al., Effect of increase of radiation dose on local control relates to pre-treatment FDG uptake in FaDu tumours in nude mice, Radiother Oncol 83 (3), 311-315 (2007).

37. Hicks RJ, Mac Manus MP, Matthews JP, et al., Early FDG-PET imaging after radical radiotherapy for non-small-cell lung cancer: inflammatory changes in normal tissues correlate with tumor response and do not confound therapeutic response evaluation, Int J Radiat Oncol Biol Phys 60 (2), 412-418 (2004).

38. Verel I, Visser GW, Boellaard R, et al., 89Zr immuno-PET: comprehensive procedures for the production of 89Zr-labeled monoclonal antibodies, J Nucl Med 44 (8), 1271-1281 (2003).

39. Huang SM, Bock JM and Harari PM, Epidermal growth factor receptor blockade with C225 modulates proliferation, apoptosis, and radiosensitivity in squamous cell carcinomas of the head and neck, Cancer Res 59 (8), 1935-1940 (1999).

40. Bonner JA, Harari PM, Giralt J, et al., Radiotherapy plus Cetuximab for Squamous-Cell Carcinoma of the Head and Neck, N Engl J Med 354 (6), 567-578 (2006).

41. Verel I, Visser GW, Boerman OC, et al., Long-lived positron emitters zirconium-89 and iodine-124 for scouting of therapeutic radioimmunoconjugates with PET, Cancer Biother Radiopharm 18 (4), 655-661 (2003).

42. Herbst RS, Review of epidermal growth factor receptor biology, Int J Radiat Oncol Biol Phys 59 (2 Suppl), 21-26 (2004).

43. Mendelsohn J, Targeting the epidermal growth factor receptor for cancer therapy, J Clin Oncol 20 (18 Suppl), 1S-13S (2002).

44. Akimoto $T$, Hunter NR, Buchmiller L, et al., Inverse relationship between epidermal growth factor receptor expression and radiocurability of murine carcinomas, Clin Cancer Res 5 (10), 2884-2890 (1999).

45. Eriksen JG, Steiniche T and Overgaard J, The influence of epidermal growth factor receptor and tumor differentiation on the response to accelerated radiotherapy of squamous cell carcinomas of the head and neck in the randomized DAHANCA 6 and 7 study, Radiother Oncol 74 (2), 93-100 (2005).

46. Huang SM and Harari PM, Modulation of radiation response after epidermal growth factor receptor blockade in squamous cell carcinomas: inhibition of damage repair, cell cycle kinetics, and tumor angiogenesis, Clin Cancer Res 6 (6), 2166-2174 (2000).

47. Lammering $\mathrm{G}$, Molecular predictor and promising target: will EGFR now become a star in radiotherapy?,Radiother Oncol 74 (2), 89-91 (2005).

48. Hebbar M, Wacrenier A, Desauw C, et al., Lack of usefulness of epidermal growth factor receptor expression determination for cetuximab therapy in patients with colorectal cancer, Anticancer Drugs 17 (7), 855-857 (2006).

49. Lenz HJ, Van Cutsem E, Khambata-Ford S, et al., Multicenter phase II and translational study of cetuximab in metastatic colorectal carcinoma refractory to irinotecan, oxaliplatin, and fluoropyrimidines, J Clin Oncol 24 (30), 4914-4921 (2006).

50. Chung KY, Shia J, Kemeny NE, et al., Cetuximab shows activity in colorectal cancer patients with tumors that do not express the epidermal growth factor receptor by immunohistochemistry, J Clin Oncol 23 (9), 1803-1810 (2005). 
51. Aerts HJ, Dubois L, Hackeng TM, et al., Development and evaluation of a cetuximab-based imaging probe to target EGFR and EGFRvIII, Radiother Oncol 83 (3), 326-332 (2007).

52. Divgi $C R$, Welt $S$, Kris $M$, et al., Phase I and imaging trial of indium 111-labeled antiepidermal growth factor receptor monoclonal antibody 225 in patients with squamous cell lung carcinoma, J Natl Cancer Inst 83 (2), 97-104 (1991).

53. van Dongen GA, Visser GW, Lub-de Hooge MN, et al., Immuno-PET: A Navigator in Monoclonal Antibody Development and Applications, Oncologist 12 (12), 1379-1389 (2007).

54. Schechter NR, Yang DJ, Azhdarinia A, et al., Assessment of epidermal growth factor receptor with 99mTc-ethylenedicysteine-C225 monoclonal antibody, Anticancer Drugs 14 (1), 49-56 (2003).

55. Cai W, Chen K, He L, et al., Quantitative PET of EGFR expression in xenograft-bearing mice using (64)Cu-labeled cetuximab, a chimeric anti-EGFR monoclonal antibody, Eur J Nucl Med Mol Imaging (2007).

56. Schechter NR, Wendt RE, 3rd, Yang DJ, et al., Radiation dosimetry of 99mTc-labeled C225 in patients with squamous cell carcinoma of the head and neck, J Nucl Med 45 (10), 16831687 (2004).

57. Ping Li W, Meyer LA, Capretto DA, et al., Receptor-Binding, Biodistribution, and Metabolism Studies of (64)Cu-DOTA-Cetuximab, a PET-Imaging Agent for Epidermal Growth-Factor Receptor-Positive Tumors, Cancer Biother Radiopharm 23 (2), 158-171 (2008).

58. Goldenberg A, Masui H, Divgi $C$, et al., Imaging of human tumor xenografts with an indium111-labeled anti-epidermal growth factor receptor monoclonal antibody, J Natl Cancer Inst 81 (21), 1616-1625 (1989).

59. Verel I, Visser GW, Boellaard R, et al., Quantitative 89Zr immuno-PET for in vivo scouting of 90Y-labeled monoclonal antibodies in xenograft-bearing nude mice, J Nucl Med 44 (10), 1663-1670 (2003).

60. Perk LR, Visser GW, Vosjan MJ, et al., (89)Zr as a PET surrogate radioisotope for scouting biodistribution of the therapeutic radiometals (90)Y and (177) Lu in tumor-bearing nude mice after coupling to the internalizing antibody cetuximab, J Nucl Med 46 (11), 1898-1906 (2005).

61. Verel I, Visser GW, Vosjan MJ, et al., High-quality 124I-labelled monoclonal antibodies for use as PET scouting agents prior to 131।-radioimmunotherapy, Eur J Nucl Med Mol Imaging 31 (12), 1645-1652 (2004).

62. Nagengast WB, de Vries EG, Hospers GA, et al., In vivo VEGF imaging with radiolabeled bevacizumab in a human ovarian tumor xenograft, J Nucl Med 48 (8), 1313-1319 (2007).

63. Borjesson PK, Jauw YW, Boellaard R, et al., Performance of immuno-positron emission tomography with zirconium-89-labeled chimeric monoclonal antibody U36 in the detection of lymph node metastases in head and neck cancer patients, Clin Cancer Res 12 (7 Pt 1), 2133-2140 (2006).

64. Perk LR, Visser OJ, Stigter-van Walsum M, et al., Preparation and evaluation of (89)ZrZevalin for monitoring of (90)Y-Zevalin biodistribution with positron emission tomography, Eur J Nucl Med Mol Imaging 33 (11), 1337-1345 (2006). 


\section{CHAPTER}

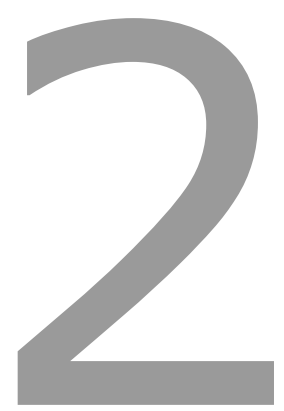

Identification of radioresistance: Validation of location residual metabolic-active areas

Published in: Radiotherapy and Oncology 91 (2009) 386-392

Identification of Residual Metabolic-active Areas within Individual NSCLC Tumours using a PreRadiotherapy ${ }^{18}$ Fluorodeoxyglucose-PET-CT Scan

Hugo J.W.L. Aerts, Angela A.W. van Baardwijk, Steven F. Petit, Claudia Offermann, Judith van Loon, Ruud Houben, Anne-Marie C. Dingemans, Rinus Wanders, Liesbeth Boersma, Jack Borger, Gerard Bootsma, Wiel Geraedts, Cordula Pitz, Jean Simons, Bradly G. Wouters, Michel Oellers, Philippe Lambin, Geert Bosmans, Andre L.A.J. Dekker, Dirk De Ruysscher 


\section{ABSTRACT}

\section{Background and Purpose}

Non-small cell lung cancer (NSCLC) tumours are mostly heterogeneous. We hypothesized that areas within the tumour with a high pre-radiation ${ }^{18} \mathrm{~F}$-deoxyglucose (FDG) uptake, could identify residual metabolic-active areas, ultimately enabling selectiveboosting of tumour sub-volumes.

\section{Material and Methods}

Fifty-five patients with inoperable stage I-III NSCLC treated with chemo-radiation or with radiotherapy alone were included. For each patient one pre-radiotherapy and one post-radiotherapy FDG-PET-CT scans was available. Twenty-two patients showing persistent FDG-uptake in the primary tumour after radiotherapy were analyzed. Overlapfractions (OF) were calculated between standardized uptake value (SUV) thresholdbased auto-delineations on the pre- and post-radiotherapy scan.

\section{Results}

Patients with residual metabolic-active areas within the tumour had a significantly worse survival compared to individuals with a complete metabolic response $(p=0.002)$. The residual metabolic-active areas within the tumour largely corresponded (OF>70\%) with the 50\%SUV high FDG-uptake area of the pre-radiotherapy scan. The hotspot within the residual area (90\%SUV) was completely within the GTV (OF=100\%), and had a high overlap with the pre-radiotherapy $50 \%$ SUV threshold (OF>84\%).

\section{Conclusion}

The location of residual metabolic-active areas within the primary tumour after therapy corresponded with the original high FDG-uptake areas pre-radiotherapy. Therefore, a single pre-treatment FDG-PET-CT scan allows for the identification of residual metabolic-active areas. 


\section{INTRODUCTION}

Lung cancer still remains one of the most frequent and lethal solid tumours ${ }^{1}$. Although the prognosis has improved, in locally advanced non-small cell lung cancer (NSCLC), the 5 -year survival is $20 \%$ at best ${ }^{2-7}$. As the improved long-term survival with concurrent chemo-radiation compared to sequential chemotherapy and radiotherapy is due to improved local tumour control, strategies to increase local tumour control are warranted ${ }^{6-8}$. Local tumour failure is indeed still observed in about $70 \%$ of patients ${ }^{5-7}$.

An important strategy to improve local tumour control is to escalate the radiation dose, because a higher dose has been shown to yield a higher local control rate ${ }^{9-11}$. However, radiation dose increase is limited by the toxicity of radiation to normal tissues such as the lungs and the spinal cord. Although accelerated, high-dose schedules based on normal tissue constraints have shown promising results ${ }^{12-14}$, innovative strategies are needed to be able to deliver doses up to $120 \mathrm{~Gy}$, which are needed to obtain local tumour control rates over $90 \%{ }^{15}$. A possibility to achieve this goal would be to take advantage of intra-tumour heterogeneity. It has become increasingly clear, that a tumour is heterogeneous for varying characteristics, possibly also for radioresistance ${ }^{16,17}$. Indeed, molecular imaging studies showed significant differences in perfusion, hypoxia, cell density and proliferation within the tumour ${ }^{18-22}$. As the tumour is thus probably composed of areas with different radio-resistance, a strategy to deliver a non-uniform dose-distribution seems logical. It appears to be a way to design volumetric maps of radio-resistance using molecular imaging and to redistribute the radiation dose according to this. More resistant areas within the tumour could thus receive higher doses whilst reducing the dose to more susceptible zones with the same normal tissue exposure ${ }^{18}$.

${ }^{18} \mathrm{~F}$-deoxyglucose (FDG) is a commonly used marker in oncology for the assessment of glucose metabolism ${ }^{23}$. FDG uptake in the primary tumour before treatment is prognostic for survival in patients with NSCLC, both treated with surgery and radiotherapy 24-28. The metabolic response of patients after radiotherapy or chemo-radiation is correlated with survival ${ }^{29}$. In a pre-clinical investigation an increase of radiation dose showed a higher local control for tumours with higher FDG uptake compared to tumours with lower FDG uptake ${ }^{17}$. Therefore, we hypothesized that the high FDG uptake locations before treatment could prossibly identify more radioresistant areas within the tumour. If true, an increase survival could be expected from radiation dose redistribution according to FDG uptake pre-radiotherapy. In an earlier study, we demonstrated that for NSCLC, the high-uptake areas of FDG within the tumour remained stable during a course of fractionated radiotherapy for $\mathrm{NSCLC}^{30}$, being a prerequisite for selective radiation boosting of these zones. In the present study, we investigated 
whether the high FDG uptake area within the primary tumour before treatment indeed identifies the location of the residual metabolic-active areas after treatment.

\section{METHODS}

\section{Patient Characteristics}

Fifty-five patients (16 women and 39 men) with inoperable non-small cell lung cancer (NSCLC), UICC stage I-III, treated with radical radiotherapy (RT) alone (11 patients) or with sequential chemo-radiotherapy (44 patients) were studied as part of two phase II trials (NCT00573040, NCT00572325). No concurrent chemo-radiotherapy was given. The sequential chemo-radiotherapy schedule consisted of 3 cycles of carboplatin or cisplatin and gemcitabine before the start of RT. Patients were included from January 2005 until February 2007. Mean age was 65.4 years (range: $44-83$ years). The Medical Ethics Committee according to the Dutch law approved the trial. All patients gave written informed consent before entering the studies. Two FDG-PET-CT scans were available for each patient. The first scan was performed on average 12.8 days (range: 1-56 days) before start of RT while the second scan was performed 86.1 days (range: 49-184 days) after the end of RT.For the patients receiving sequential chemo-radiotherapy was the pre-RT scan performed after the chemotherapy. No treatment was given to any of the patients between the end of RT and the post-RT scan.

\section{Radiotherapy Simulation}

Patients were simulated in radiotherapy position on a dedicated PET-CT-simulator with both arms above the head. For the FDG-PET-CT scans a Siemens Biograph (SOMATOM Sensation-16 with an ECAT ACCEL PET scanner) was used. An intravenous injection of (weight * $4+20$ ) MBq FDG (Tyco Health Care, Amsterdam, The Netherlands) was followed by $10 \mathrm{ml}$ physiologic saline. After a 45 minutes uptake period, during which the patient was encouraged to rest, PET and CT images were acquired. A spiral CT ( $3 \mathrm{~mm}$ slice thickness) with intravenous contrast was performed covering the complete thoracic region.

\section{Radiotherapy Planning}

Radiotherapy planning was performed on a XiO (Computerized Medical Systems, St Louis, Missouri) treatment planning system, based on a convolution algorithm using inhomogeneity corrections. The Gross Tumour Volume (GTV) and the Planning Target Volume (PTV) were defined for all patients, based on PET-CT data ${ }^{13}$. The Clinical Target Volume (CTV) was defined as the GTV with a $5 \mathrm{~mm}$ margin incorporating microscopic disease. Subsequently, this CTV was expanded with $1 \mathrm{~cm}$ to draw the PTV to incorpo- 
rate the internal respiratory motion and setup errors. Contouring of the lungs was carried out automatically by the treatment planning system. The volume of both lungs excluding the GTV was used for the calculation of the mean lung dose (MLD). The spinal cord was drawn throughout the whole CT scan, considered to be at the inner margin of the bony spinal canal. A 3D conformal treatment plan was calculated on the PTV for all patients according to ICRU 50 guidelines ${ }^{31}$. Dosimetric values were calculated on the basis of dose-volume histograms and dose distributions on each axial CT plan. For each patient, the radiation dose was escalated to an individualized maximal total tumour dose, applying an MLD of $19 \mathrm{~Gy}$ while respecting a maximum spinal cord dose of $54 \mathrm{~Gy}^{14}$. The maximal total tumour dose allowed was $79.2 \mathrm{~Gy}$. There were no esophageal dose constraints. Radiotherapy was delivered twice a day, 5 days per week, with a minimum of 8 hours between the two fractions. In all patients, individualized patient dosimetry using electronic portal imaging devices was performed ${ }^{32}$.

Pre-radiotherapy scan:

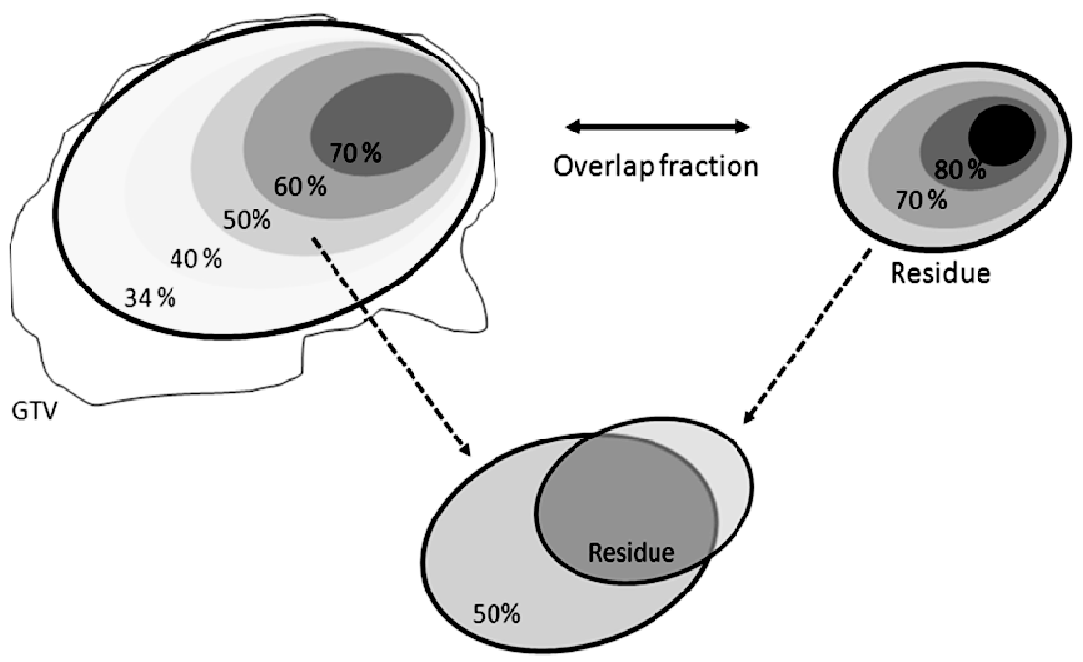

overlap fraction (OF) $=\frac{\text { overlapping area }}{\text { smallest volume }}$

\section{Figure 1}

Schematic representation of the overlap-fractions (OF) quantification of FDG-uptake preradiotherapy with residual metabolic-active areas post-radiotherapy. Within the preradiotherapy GTV (defined with the knowledge of the FDG-PET-CT scan), the FDG-PET uptake areas were quantified (34-70\%SUVmax). Within the residual areas, the high FDG-uptake areas (70-90\%SUVmax) were quantified. As an example, the OF of the 50\%SUVmax FDG high-uptake zone pre-radiotherapy with the residue was illustrated (OF about $70 \%$ ). 


\section{Image Analysis}

The pre- and post-RT scans were analyzed and delineated using the Siemens TrueD system (Version VC-30, Siemens A.G., Darmstad, Germany). Descriptively, a schematic representation of this methodology is shown in Figure 1. The location and volume of the FDG uptake areas pre-RT were quantified within the primary tumour using the threshold $34,40,50,60$ and $70 \%$ of the maximal SUV $\left(S_{\text {max }}\right)$. Residual metabolic areas were defined as FDG uptake higher than in the aortic arch $\left(S U V>S U V_{\text {aorta }}\right)^{29}$. Within the residual FDG-positive areas on the post-RT scan, the high FDG uptake areas were defined using the thresholds 70,80 , and $90 \%$ SUV $_{\max }$. Also the fixed thresholds, SUV 2.5 and SUV 5.0, of the residual disease were delineated on the post-RT scan (not illustrated). Using an automatic rigid registration algorithm based on mutual information of the CT scans, the images of pre-RT scan were fused to the post-RT scan on the Siemens TrueD system. If the automatic registration showed a large deformation between the two CT scans, the images were manually registered on the surrounding anatomy of the tumour, e.g. the bony anatomy or great vessels. The contour delineations on the post-RT scan were then transformed to the pre-RT scan using the derived registration matrices. The contour delineations were exported from TrueD as DICOM-RT structure sets. Using MATLAB 7.1 SP3 (The MathWorks Inc, Natick, MA, USA) the overlap fractions (OF) and volumes of these FDG based delineations were calculated. The overlap fraction was defined as the volume of overlap divided by the smallest volume ${ }^{30}$. See figure 1 for a schematic representation of this calculation. By using this method it is possible to assess which threshold on the pre-RT scan matches the residual disease on the post-RT scan.

\section{Statistical Analysis}

All data are expressed as mean $\pm 95 \%$ confidence intervals $(95 \% \mathrm{Cl})$. Statistical differences between the parameters were evaluated in SPSS (Version 15.0 for Windows, Chicago, IL), using the Mann-Whitney $U$ test. Differences were considered to be significant when the $p$-value was less than 0.05. A power calculation was performed to assess the power of the patient group for the metabolic-active areas located at the FDG hotspot vs. at random ${ }^{33}$.The overall survival of the patients and the $95 \% \mathrm{Cl}$ were calculated using the Kaplan-Meier method. Differences between the groups were assessed using the log-rank test. The Cox regression method was used to estimate the hazard ratio. 


\begin{tabular}{|c|c|c|c|c|c|c|c|c|c|c|c|c|c|}
\hline 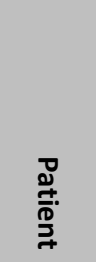 & 觉 & $\begin{array}{l}\text { Q } \\
\frac{D}{2} \\
\frac{0}{D}\end{array}$ & 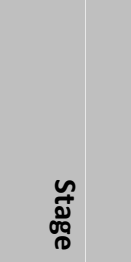 & $\begin{array}{l}\exists \\
\text { ㄱ. } \\
\text { ล }\end{array}$ & $\begin{array}{l}\text { 엌 } \\
\frac{1}{a} \\
\sum_{n=0}^{2}\end{array}$ & 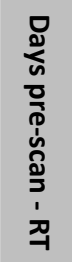 & 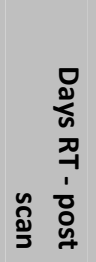 & 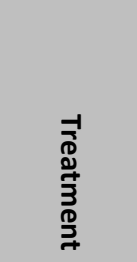 & 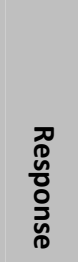 & 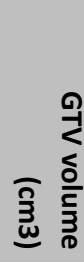 & 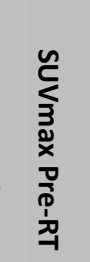 & 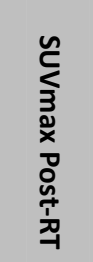 & 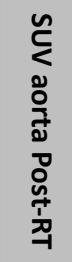 \\
\hline 1 & 54 & $\mathrm{M}$ & T3N2M0 & 56 & 22 & 9 & 96 & seq CT-RT & SMD & 152 & 6.68 & 5.77 & 3.53 \\
\hline 2 & 72 & $M$ & T2N3M0 & 72 & 28 & 7 & 64 & seq CT-RT & PMR & 72 & 8.80 & 3.50 & 2.03 \\
\hline 3 & 54 & $M$ & T2N3M0 & 79 & 30 & 7 & 49 & seq CT-RT & PMR & 77 & 10.31 & 5.35 & 3.25 \\
\hline 4 & 64 & $\mathrm{M}$ & T4NOMO & 79 & 20 & 12 & 76 & seq CT-RT & PMR & 29 & 5.50 & 3.60 & 2.90 \\
\hline 5 & 61 & $\mathrm{~F}$ & $\mathrm{~T} 2 \mathrm{~N} 2 \mathrm{MO}$ & 47 & 17 & 8 & 89 & seq CT-RT & PMR & 272 & 7.70 & 4.10 & 2.54 \\
\hline 6 & 72 & $\mathrm{M}$ & T3N2M1 & 70 & 27 & 28 & 122 & seq CT-RT & PMR & 87 & 15.65 & 5.78 & 2.45 \\
\hline 7 & 83 & $M$ & T2NOMO & 79 & 32 & 8 & 102 & RT & PMR & 122 & 10.61 & 7.39 & 3.26 \\
\hline 8 & 79 & $\mathrm{M}$ & $\mathrm{T} 2 \mathrm{~N} 2 \mathrm{MO}$ & 72 & 28 & 12 & 92 & seq CT-RT & PMR & 55 & 10.40 & 6.70 & 1.84 \\
\hline 9 & 83 & $M$ & T2N1M0 & 59 & 22 & 8 & 106 & RT & SMD & 87 & 9.63 & 11.94 & 2.96 \\
\hline 10 & 58 & $\mathrm{~F}$ & T3N2M0 & 54 & 20 & 14 & 77 & seq CT-RT & SMD & 104 & 18.69 & 17.37 & 3.67 \\
\hline 11 & 63 & $M$ & T2NOMO & 54 & 21 & 56 & 88 & RT & PMR & 26 & 8.41 & 4.14 & 2.78 \\
\hline 12 & 44 & $\mathrm{~F}$ & $\mathrm{~T} 2 \mathrm{~N} 2 \mathrm{M} 0$ & 76 & 30 & 8 & 112 & seq CT-RT & SMD & 20 & 4.50 & 4.20 & 3.40 \\
\hline 13 & 77 & $\mathrm{M}$ & T4NOMO & 54 & 25 & 12 & 99 & seq CT-RT & PMR & 125 & 19.70 & 3.60 & 2.50 \\
\hline 14 & 63 & $\mathrm{~F}$ & $\mathrm{~T} 2 \mathrm{~N} 2 \mathrm{MO}$ & 58 & 21 & 13 & 76 & seq CT-RT & SMD & 131 & 11.80 & 13.90 & 2.65 \\
\hline 15 & 80 & $\mathrm{M}$ & T2N1M0 & 54 & 22 & 41 & 70 & RT & SMD & 139 & 8.54 & 7.43 & 2.85 \\
\hline 16 & 79 & $\mathrm{~F}$ & T4NOMO & 58 & 24 & 5 & 69 & seq CT-RT & PMR & 19 & 8.50 & 5.80 & 2.95 \\
\hline 17 & 69 & $\mathrm{M}$ & $\mathrm{T} 2 \mathrm{~N} 2 \mathrm{M} 0$ & 63 & 25 & 9 & 86 & seq CT-RT & SMD & 39 & 11.91 & 11.91 & 3.65 \\
\hline 18 & 53 & $M$ & $\mathrm{~T} 2 \mathrm{~N} 2 \mathrm{MO}$ & 54 & 22 & 8 & 82 & seq CT-RT & PMR & 128 & 15.90 & 8.90 & 2.88 \\
\hline 19 & 69 & $\mathrm{M}$ & T1N2M0 & 54 & 18 & 10 & 131 & seq CT-RT & PMR & 48 & 16.02 & 7.60 & 3.10 \\
\hline 20 & 56 & $\mathrm{M}$ & T4N2M0 & 79 & 30 & 6 & 83 & seq CT-RT & PMR & 102 & 8.40 & 3.90 & 1.75 \\
\hline 21 & 73 & $\mathrm{M}$ & T4N2M0 & 79 & 32 & 7 & 66 & seq CT-RT & PMR & 148 & 4.30 & 3.30 & 2.90 \\
\hline 22 & 50 & $\mathrm{~F}$ & T4N2MO & 56 & 21 & 13 & 71 & seq CT-RT & PMR & 121 & 9.20 & 6.10 & 3.65 \\
\hline Mean: & 66.2 & & & 64.0 & 24.4 & 13.7 & 86.6 & & & 95.6 & 10.5 & 6.9 & 2.9 \\
\hline Std: & 11.5 & & & 11.0 & 4.6 & 12.4 & 19.9 & & & 58.8 & 4.3 & 3.8 & 0.6 \\
\hline $\min :$ & 44 & & & 46.8 & 17.0 & 5 & 49.0 & & & 19.0 & 4.3 & 3.3 & 1.8 \\
\hline max: & 83 & & & 79.2 & 32.0 & 56 & 131.0 & & & 272.2 & 19.7 & 17.4 & 3.7 \\
\hline
\end{tabular}

RT: radiotherapy; M: male; F: female; TTD: total tumour dose; OTT: overall treatment time of radiotherapy; post scan: FDG-PET-CT scan after the end of radiotherapy; RT: radiotherapy alone; seq CT-RT: sequential chemotherapy and radiotherapy; Response (EORTC criteria): SMD: stable metabolic disease PMR: partial metabolic response; GTV: gross tumour volume 


\section{RESULTS}

\section{Patient Characteristics}

To assess the location of residual metabolic active areas compared with initial FDG uptake in the primary tumour, two ${ }^{18}$ Fluorodeoxyglucose (FDG) PET-CT scans were analyzed for all patients, one before radiotherapy (pre-RT) and one after radiotherapy (post-RT). Of all 55 patients, 28 showed metabolic active areas with residual FDG uptake in the proximity or within the primary tumour on the post-RT scan. The other 27 patients had a complete metabolic response, showing no residual metabolic activity in the primary tumour. Both patients with and without residual areas showed heterogeneous FDG uptake patterns within the macroscopic tumour before treatment, where the high uptake area was not necessarily in the center of the tumour. The maximum FDG uptake on the pre-RT scan for patients with residual areas was significantly higher than for patients with a complete metabolic response $\left(\mathrm{SUV}_{\max }=9.6\right.$ [95\% Cl: 8.0-11.2] and 7.1 [95\% Cl: $5.6-8.7$ ] respectively; $p=0.029$ ). Also the GTV volume was significantly higher for patients showing residual areas (with residual areas: $117.3 \mathrm{~cm}^{3}[95 \% \mathrm{Cl}: 70.3-$ $164.3 \mathrm{~cm}^{3}$ ], without residual areas: $\left.54.7 \mathrm{~cm}^{3}\left[95 \% \mathrm{Cl}: 38.7-70.6 \mathrm{~cm}^{3}\right] ; p=0.003\right)$. The total tumour dose was not different $(p=0.547)$ from the patients without residual disease $(65 \pm 8.6 \mathrm{~Gy})$, compared to the patients with residual disease ( $64.3 \pm 10.9 \mathrm{~Gy})$. Also the overall treatment time was similar for the patients without residual disease (26.5 \pm 5.8 days) compared to the patients with residual disease ( $24.8 \pm 4.7$ days) ( $p=$ 0.326). Concordant with literature ${ }^{29}$, with a median follow-up of 29.9 months, patients with residual areas had a worse survival than individuals with a complete metabolic response (12.2 months vs. median survival not reached; hazard ratio for death: 2.94 [95\%Cl: 1.44-5.99], $p=0.002$; Figure 2).

Twenty-two out of the 28 patients remained for further analysis. Three patients were excluded because the residue was not clearly distinguishable from the surrounding tissue due to FDG avid inflammation, pneumonitis or to the vicinity of the heart. In addition two patients were excluded due to large deformation of anatomical structures between the pre- and post-RT scans, because no reliable registration could be performed (determined by two independent observers). One patient with progressive disease was excluded because the residual disease was larger than the primary tumour, excluding reliable calculation of overlap fractions. The characteristics of these patients are shown in Table 1. 


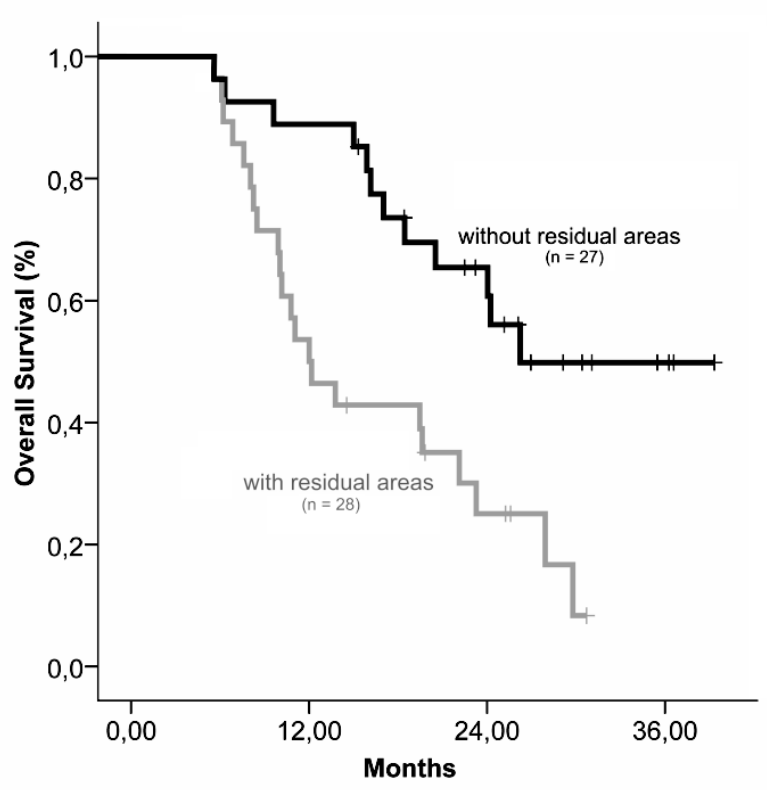

\section{Figure 2}

Kaplan-Meier estimates of overall survival of all 55 patients with residual metabolic active areas $(n=28)$ and without residual areas $(n=27)$ on the post-radiotherapy FDG-PET-CT scan. The hazard ratio for death for patients with residual areas compared to individuals without was 2.94 195\% confidence interval: 1.44 to $5.99 ; p=0.002$ by the log-rank test, two-sided).

\section{Overlap fractions between FDG-PET-CT scans pre- and post-radiotherapy}

In Figure 3, representative images are shown of three typical patients with a large homogeneous tumour (Patient 1), a large heterogeneous tumour (Patient 2) and a small tumour (Patient 3). The location of the residual areas ( $S U V>S U V_{\text {aorta }}$ ) on the post-RT scan and the high FDG uptake areas $\left(50 \%\right.$ SUV $\left._{\max }\right)$ on the pre-RT scan are shown. The residual areas are transposed to the pre-RT scan, to show the overlap with $50 \% \mathrm{SUV}_{\max }$ high uptake area pre-RT. Visual evaluation shows that the location of the residual areas largely corresponds with the high FDG uptake areas pre-RT.

\section{Volumes of the FDG based thresholds}

The volumes of the FDG based thresholds of the tumour pre- and post-RT are shown in Figure 4. The high FDG uptake areas $\left(50-70 \% \mathrm{SUV}_{\max }\right)$ within the tumour on the pre-RT scan were small compared to the GTV volume. The $50 \%$ SUV $_{\max }$ encompassed $39.0 \%$ [95\% Cl: 31.9-46.0\%] of the original GTV, whereas this was $24.9 \%$ [95\% Cl: $20.4-29.4 \%$ ] for the $60 \%$ SUV $_{\max }$, and $13.7 \%$ [95\%Cl: 11.0-16.3\%] for the $70 \% \mathrm{SUV}_{\max }$ threshold (Fig. 

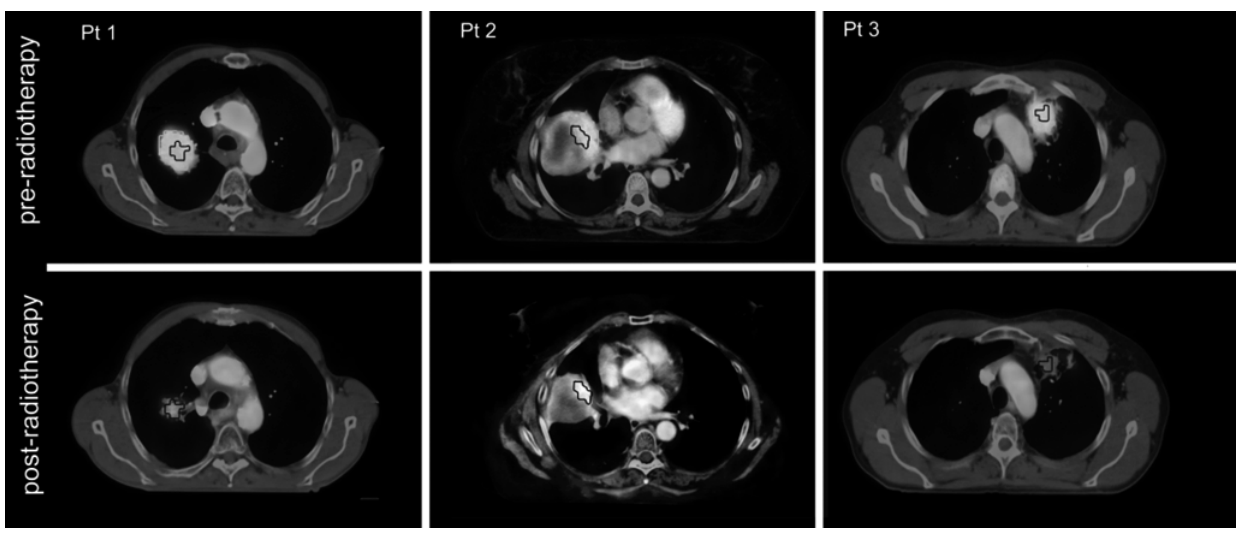

Figure 3

Representative FDG-PET-CT images of 3 patients pre- and post-radiotherapy. The light gray contours indicates the $50 \% S U V_{\max }$ FDG high uptake areas pre-radiotherapy. The drak gray contours indicate the residual metabolic active areas post-radiotherapy, also transposed on the preradiotherapy scan. Visual evaluation shows a large correspondence between the residual areas post-radiotherapy with the high FDG uptake areas pre-radiotherapy.

$4 A)$. The volume of the residual metabolic active areas on the post-RT scan was $21.7 \%$ [95\% Cl: $15.1-28.3 \%$ ] of the pre-RT GTV volume (Fig. 4B). The relative volumes of the high uptake areas within the residual areas were very small: $7.8 \%$ [95\% $\mathrm{Cl}: 4.4-11.2 \%]$ for the $70 \%$ SUV $_{\max }, 2.5 \%$ [95\% Cl: 1.5-3.5\%] for the $80 \% \mathrm{SUV}_{\max }$, and $0.8 \%$ [95\%Cl: 0.4$1.3 \%$ ] for the $90 \% \mathrm{SUV}_{\max }$ threshold. Also the absolute thresholds of the residual areas had a relative small volume: $31.5 \%$ [95\% Cl: $22.4-40.6 \%]$ and $7.0 \%$ [95\% $\mathrm{Cl}: 3.0-11.0 \%]$ for SUV2.5 and SUV5 respectively.

\section{Overlap Fractions}

Figure 5 depicts the overlap fractions of the FDG uptake within the primary tumour pre-RT with the post-RT thresholds. The residual areas were mainly located within the original GTV (OF $=91.8 \%$ [95\%Cl: 87.9-95.6\%]) (Fig. 5A). The 70\% and $80 \%$ SUV $_{\max }$ highuptake areas within the residual areas were also mainly located within the GTV (OF = 94.0\% [95\% $\mathrm{Cl}: 89.2-98.7 \%$ ] and 96.5\% [95\% $\mathrm{Cl}: 93.1-100.0 \%]$ respectively). Whereas, the $90 \%$ SUV $_{\max }$ high-uptake areas were all completely located within the GTV (OF = $100 \%)$. Comparing the pre-RT FDG uptake with the residual areas, the $34 \%$ threshold pre-RT had a large overlap fraction with the residual areas (89.3\% [95\% Cl: 84.8-93.8\%]) and with the high-uptake areas within the residue. Moreover, the same is true for the pre-RT 50\% SUV max $_{\operatorname{migh}}$ FDG uptake area (OF $=70.4 \%$ [95\% $\left.\mathrm{Cl}: 63.0-77.7 \%\right]$ ). This preRT $50 \%$ SUV $_{\text {max }}$ area also largely corresponded with the $70-90 \%$ SUV $_{\max }$ high-uptake areas within the residue. The 22 analyzable patients resulted in a power of over 0.9 (with alpha $=0.01, \mathrm{H}_{0}: 30 \%$ of patients with residue randomly located at FDG. 
A

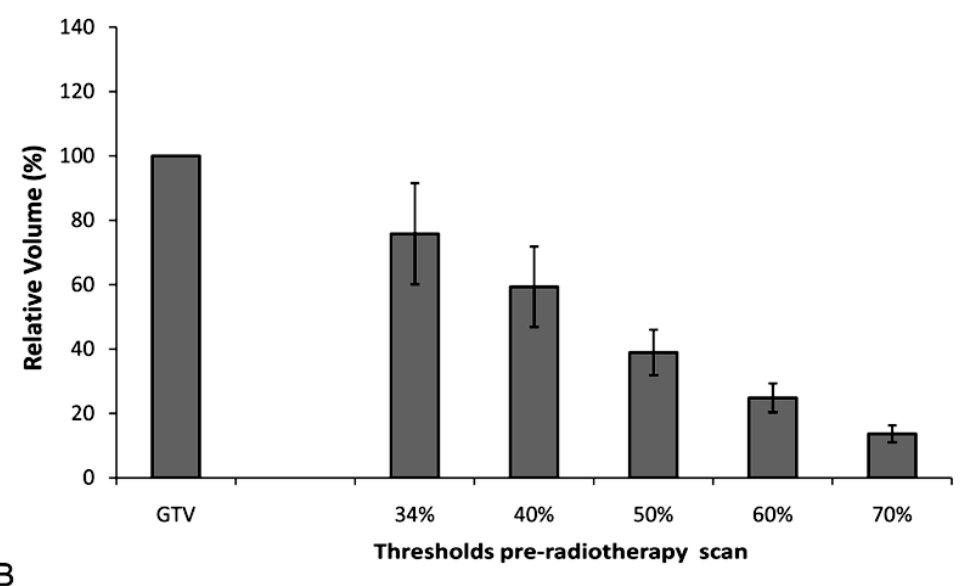

B

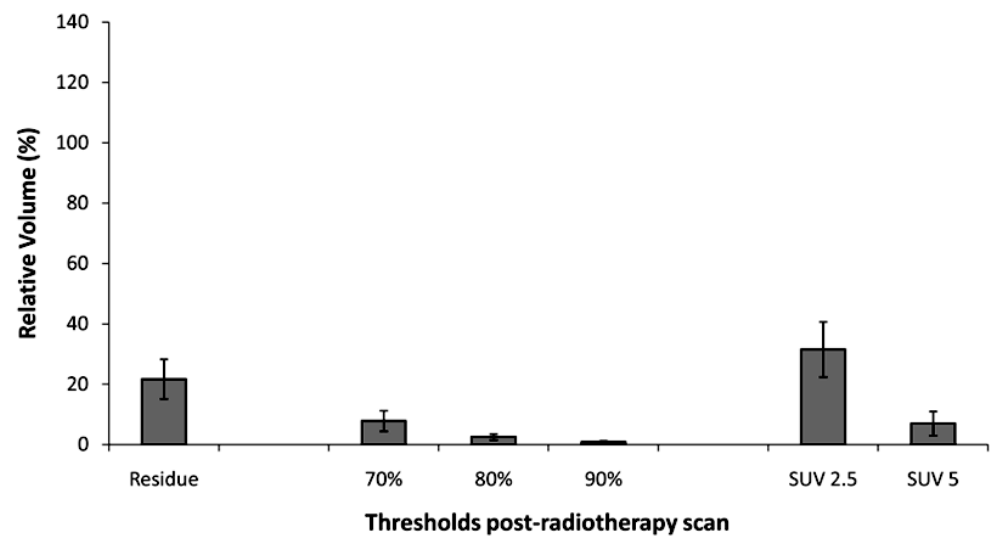

\section{Figure 4}

Volumes of the SUV thresholds of the tumour pre-radiotherapy (A) and post-radiotherapy (B). All volumes $(A$ and $B)$ are relative to the pre-radiotherapy gross tumour volume (GTV). The data are expressed as mean $\pm 95 \%$ confidence intervals (error-bars). Note that the volume of residual metabolic active areas was on average $22 \%$ of the GTV volume.

hotspot vs $\mathrm{H} 1: 70 \%$ in case of non-random location of residue).The extent of the GTV corresponded largely with the absolute SUV2.5 (OF $=85.9 \%$ [95\% $\mathrm{Cl}: 79.2-92.5 \%]$ ) and the SUV5 (OF $=96.0 \%$ [95\% Cl: 92.9-99.1\%]) threshold on the post-RT scan (Fig. 5B). Also the 34\% threshold pre-RT largely corresponded with the SUV2.5 and SUV5 thresholds post-RT (OF $=82.0 \%$ [95\%Cl: 75.9-88.1\%] and 95.4\% [95\%Cl: 91.2-97.9\%] respectively). The $50 \%$ pre-RT threshold had an OF of $67.8 \%$ [ $95 \% \mathrm{Cl}: 59.9-75.6 \%$ ] with the SUV2.5 threshold and an OF of $76.4 \%$ [95\%Cl: $65.6-87.3 \%$ ] with the SUV5 threshold. 
A

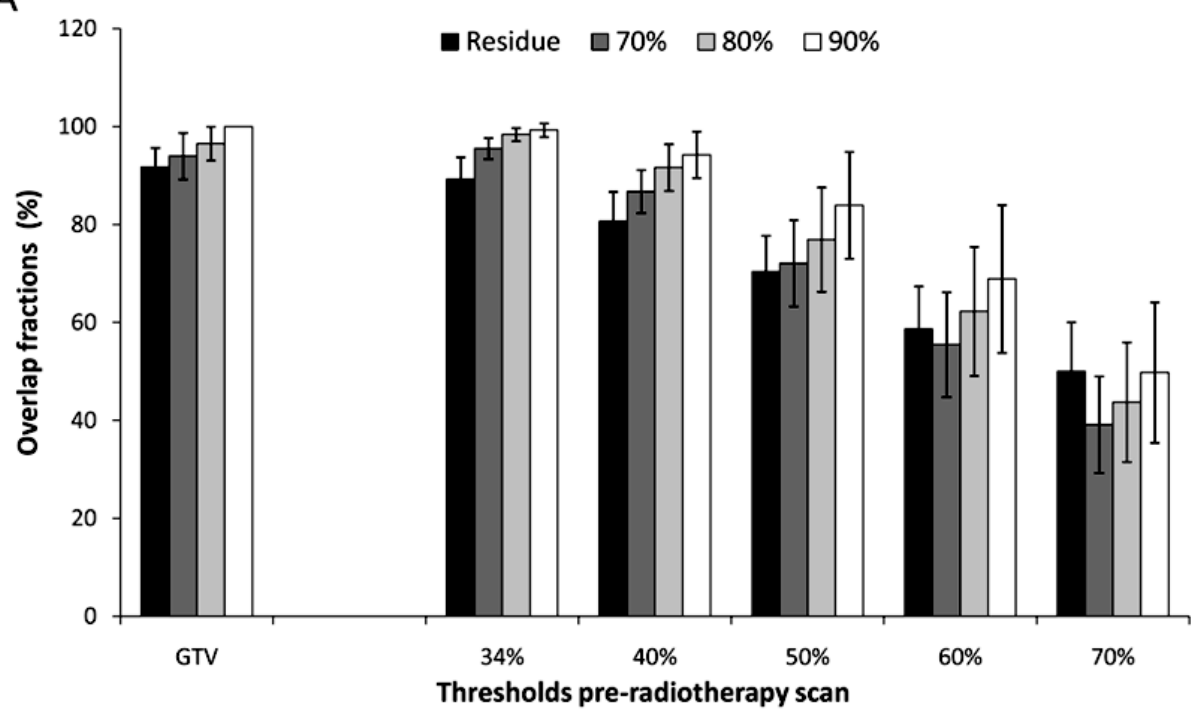

B

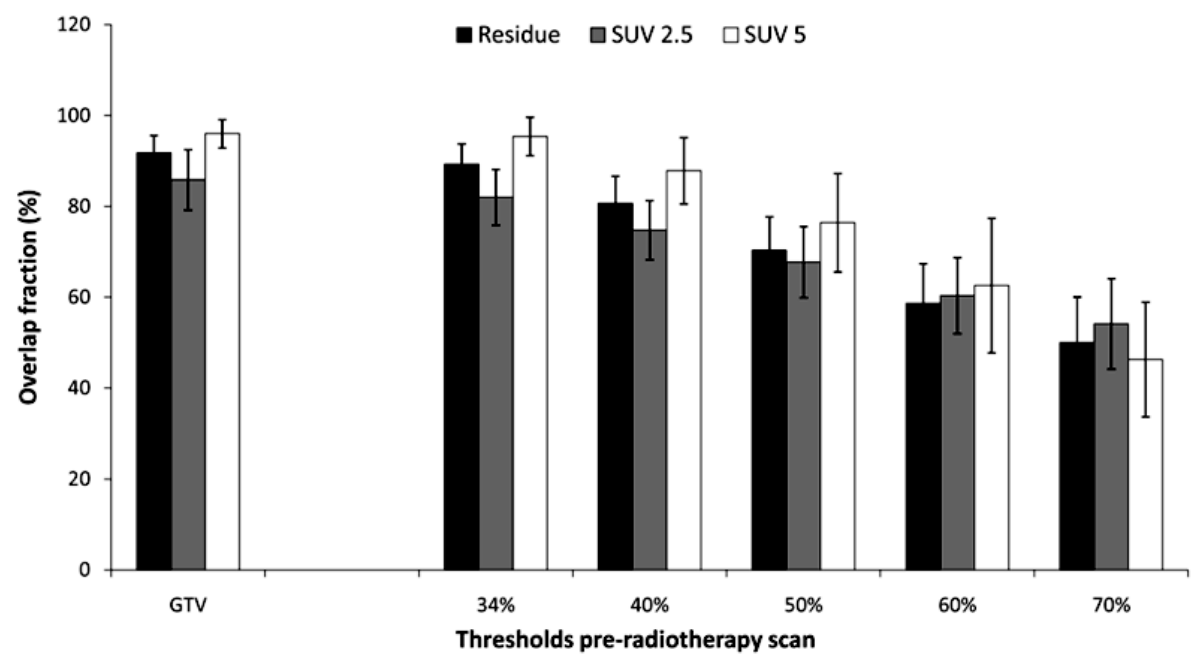

Figure 5

Overlap-fractions (OF) of the pre-radiotherapy with the post-radiotherapy relative (A) and abso-

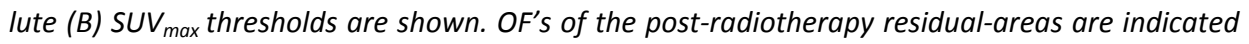
with the black-bars. The other bars indicate the OF with the high FDG-uptake areas within the residue $(A)$ and absolute thresholds (B). The data are expressed as mean $\pm 95 \%$ confidenceintervals. 


\section{DISCUSSION}

There is a growing interest in radiation oncology to selectively target radio-resistant areas with a high probability of persisting tumour cells after treatment within the tumour ${ }^{18,34,35}$. Indeed, by selectively boosting radio-resistant areas whilst decreasing the dose to more susceptible zones, local tumour control rates could increase without increased side effects. The availability of molecular imaging techniques that enable visualization and quantification of areas with different characteristics within the tumour makes this a feasible strategy. FDG as a PET tracer is of particular interest in this respect because first, its maximal uptake in the tumour is prognostic for survival of patients with NSCLC, both treated with surgery or radiotherapy ${ }^{24-28}$. Second, our group has previously shown that the FDG uptake patterns within the tumour remain stable throughout a radiotherapy course ${ }^{30}$, and third FDG is widely available.

We hypothesized that areas of high FDG uptake within the tumour before treatment would allow identification of residual metabolic-areas after therapy. Moreover, in most tumours, a heterogeneous FDG uptake pattern before treatment is observed, where the high FDG uptake areas were often not in the centre of the tumour. This opens the potential prospect for clinically relevant radiation dose redistribution within the tumour. However, at first it has to be established whether resistant areas within the tumour can be defined on the basis of pre-treatment imaging. As a first step, we investigated the patterns of residual metabolic areas (based on FDG uptake) within the primary tumour after high-dose radiotherapy, mostly preceded by chemotherapy. In 28 out of 55 patients, FDG persisted in the areas with a high uptake before radiotherapy. The residual areas were located almost completely (OF > 91\%) within the GTV. The residual FDG positive areas largely corresponded (OF > 70\%) with the 50\% SUV high uptake area of the pre-RT scan. The average volume of this $50 \%$ SUV threshold was $39 \%$ of the original GTV volume. The volume of the residual FDG-positive areas was on average $22 \%$ of the GTV volume. The hotspot within the residual area (90\%SUV) was completely within the GTV (OF=100\%), and had a high overlap with the pre-radiotherapy $50 \%$ SUV threshold (OF>84\%). On basis of these findings, we conclude that residual metabolic active areas within the tumour can be identified using a single pre-RT FDG-PET-CT scan. A quantitative voxel-based analysis between the metabolic state of a tumour voxel after treatment and pre-treatment parameters, such as dose and GTV volume, was analyzed by Petit et al. $^{36}$.

Some limitations to our study should be addressed. First, of the 55 included patients, 28 had residual FDG uptake, of which $6(21 \%)$ had to be excluded because of progressive disease ( 1 patient), large tumour deformation ( 2 patients) or because FDG-uptake in the surrounding tissues made accurate delineation of the tumour area impossible ( 3 patients). Improvements in deformation analysis and better delineation methods are 
needed to include these patients in the analysis. Second, the $50 \%$ of SUV $\max$ threshold value was found to be the most suitable amongst several other threshold levels. This threshold was not only chosen because it yielded good results, but also because it is a simple and reproducible method and the delineation software is available in clinical settings. A lower threshold would result in boosting the entire tumour and not only the most resistant areas, whereas a too high threshold would lead to only a few voxels to be treated, which is difficult to radiate with the equipment used currently. Third, our surrogate endpoint, persistent FDG uptake in the tumour, should not be regarded as equivalent to tumour persistence, nor does metabolic complete response equals cure. However, in agreement with literature ${ }^{29}$, the present data show that survival of patients with persistent FDG uptake is significantly worse than those without, underlining its clinical validity. Fourth, for the image registration between the pre and post-scans a regid registration was performed, not incoorperating deformable tissue changes, possibly induced by the delivered therapy. This could be improved by using deformable registration techniques. However, these are difficult to validate and the reproducibility, especially in different institutes, is limited.

We show in clinical data that high FDG areas within the tumour before radiotherapy can identify areas with residual metabolic-activity, which are probably more radioresistant. Due to the spatial resolution of the FDG-PET imaging, this could only be performed on a macroscopic scale, i.e. voxel level. However, carefully designed future trails should provide the basis to test the assumption if FDG uptake reflects "radioresistance", by boosting high FDG uptake areas. It obviously may be argued that the FDG uptake in the tumour does not reflect a single biological characteristic of the tumour, but is influenced by many pathways that are related to therapy-resistance ${ }^{27}$. Indeed, more specific tracers, such as ${ }^{18} \mathrm{~F}$-misonidazol, may be useful for dose-painting within the tumour as well ${ }^{37}$.

In conclusion, our results show that the residual metabolic-active areas within the tumour after radiotherapy of chemo-radiation, is located in the high FDG uptake areas before therapy and can be delineated. This will be the basis for new clinical studies with dose redistributions according to pre-radiotherapy FDG uptake. 


\section{REFERENCES}

1. Jemal A, Siegel R, Ward E, et al., Cancer statistics, 2008, CA Cancer J Clin 58 (2), 71-96 (2008).

2. Betticher DC, Hsu Schmitz SF, Totsch M, et al., Prognostic factors affecting long-term outcomes in patients with resected stage IIIA pN2 non-small-cell lung cancer: 5-year follow-up of a phase II study, Br J Cancer 94 (8), 1099-1106 (2006).

3. van Meerbeeck JP, Kramer GW, Van Schil PE, et al., Randomized controlled trial of resection versus radiotherapy after induction chemotherapy in stage IIIA-N2 non-small-cell lung cancer, J Natl Cancer Inst 99 (6), 442-450 (2007).

4. Garrido $P$, Gonzalez-Larriba JL, Insa A, et al., Long-term survival associated with complete resection after induction chemotherapy in stage IIIA (N2) and IIIB (T4NO-1) non small-cell lung cancer patients: the Spanish Lung Cancer Group Trial 9901, J Clin Oncol 25 (30), 47364742 (2007).

5. Furuse K, Fukuoka M, Kawahara M, et al., Phase III study of concurrent versus sequential thoracic radiotherapy in combination with mitomycin, vindesine, and cisplatin in unresectable stage III non-small-cell lung cancer, J Clin Oncol 17 (9), 2692-2699 (1999).

6. Zatloukal P, Petruzelka L, Zemanova M, et al., Concurrent versus sequential chemoradiotherapy with cisplatin and vinorelbine in locally advanced non-small cell lung cancer: a randomized study, Lung Cancer 46 (1), 87-98 (2004).

7. Fournel $\mathrm{P}$, Robinet $\mathrm{G}$, Thomas $\mathrm{P}$, et al., Randomized phase III trial of sequential chemoradiotherapy compared with concurrent chemoradiotherapy in locally advanced non-small-cell lung cancer: Groupe Lyon-Saint-Etienne d'Oncologie Thoracique-Groupe Francais de Pneumo-Cancerologie NPC 95-01 Study, J Clin Oncol 23 (25), 5910-5917 (2005).

8. Schaake-Koning C, van den Bogaert W, Dalesio O, et al., Effects of concomitant cisplatin and radiotherapy on inoperable non-small-cell lung cancer, N Engl J Med 326 (8), 524-530 (1992).

9. Bradley J, A review of radiation dose escalation trials for non-small cell lung cancer within the Radiation Therapy Oncology Group, Semin Oncol 32 (2 Suppl 3), S111-113 (2005).

10. Rojas AM, Lyn BE, Wilson EM, et al., Toxicity and outcome of a phase II trial of taxanebased neoadjuvant chemotherapy and 3-dimensional, conformal, accelerated radiotherapy in locally advanced nonsmall cell lung cancer, Cancer 107 (6), 1321-1330 (2006).

11. Hayman JA, Martel MK, Ten Haken RK, et al., Dose escalation in non-small-cell lung cancer using three-dimensional conformal radiation therapy: update of a phase I trial, J Clin Oncol 19 (1), 127-136 (2001).

12. Belderbos JS, Heemsbergen WD, De Jaeger K, et al., Final results of a Phase I/II dose escalation trial in non-small-cell lung cancer using three-dimensional conformal radiotherapy, Int J Radiat Oncol Biol Phys 66 (1), 126-134 (2006).

13. De Ruysscher D, Wanders R, van Haren E, et al., HI-CHART: a phase I/II study on the feasibility of high-dose continuous hyperfractionated accelerated radiotherapy in patients with inoperable non-small-cell lung cancer, Int J Radiat Oncol Biol Phys 71 (1), 132-138 (2008).

14. van Baardwijk A, Bosmans $G$, Boersma L, et al., Individualized radical radiotherapy of nonsmall-cell lung cancer based on normal tissue dose constraints: a feasibility study, Int J Radiat Oncol Biol Phys 71 (5), 1394-1401 (2008). 
15. Fowler JF, Tome WA, Fenwick JD, et al., A challenge to traditional radiation oncology, Int J Radiat Oncol Biol Phys 60 (4), 1241-1256 (2004).

16. Cooper RA, Carrington BM, Loncaster JA, et al., Tumour oxygenation levels correlate with dynamic contrast-enhanced magnetic resonance imaging parameters in carcinoma of the cervix, Radiother Oncol 57 (1), 53-59 (2000).

17. Schutze C, Bergmann R, Yaromina A, et al., Effect of increase of radiation dose on local control relates to pre-treatment FDG uptake in FaDu tumours in nude mice, Radiother Oncol 83 (3), 311-315 (2007).

18. Bentzen SM, Theragnostic imaging for radiation oncology: dose-painting by numbers, Lancet Oncol 6 (2), 112-117 (2005).

19. Vanderstraeten B, Duthoy W, De Gersem W, et al., [18F]fluoro-deoxy-glucose positron emission tomography ([18F]FDG-PET) voxel intensity-based intensity-modulated radiation therapy (IMRT) for head and neck cancer, Radiother Oncol 79 (3), 249-258 (2006).

20. Tanderup K, Olsen DR and Grau C, Dose painting: art or science?,Radiother Oncol 79 (3), 245-248 (2006).

21. Piert M, Machulla HJ, Picchio $M$, et al., Hypoxia-specific tumor imaging with $18 \mathrm{~F}$ fluoroazomycin arabinoside, J Nucl Med 46 (1), 106-113 (2005).

22. Foo SS, Abbott DF, Lawrentschuk N, et al., Functional imaging of intratumoral hypoxia, Mol Imaging Biol 6 (5), 291-305 (2004).

23. Phelps $\mathrm{ME}$, Inaugural article: positron emission tomography provides molecular imaging of biological processes, Proc Natl Acad Sci U S A 97 (16), 9226-9233 (2000).

24. Borst GR, Belderbos JS, Boellaard R, et al., Standardised FDG uptake: a prognostic factor for inoperable non-small cell lung cancer, Eur J Cancer 41 (11), 1533-1541 (2005).

25. Downey RJ, Akhurst T, Gonen M, et al., Preoperative F-18 fluorodeoxyglucose-positron emission tomography maximal standardized uptake value predicts survival after lung cancer resection, J Clin Oncol 22 (16), 3255-3260 (2004).

26. Eschmann SM, Friedel G, Paulsen F, et al., Is standardised (18)F-FDG uptake value an outcome predictor in patients with stage III non-small cell lung cancer?, Eur J Nucl Med Mol Imaging 33 (3), 263-269 (2006).

27. van Baardwijk A, Dooms C, van Suylen RJ, et al., The maximum uptake of (18)Fdeoxyglucose on positron emission tomography scan correlates with survival, hypoxia inducible factor-1alpha and GLUT-1 in non-small cell lung cancer, Eur J Cancer 43 (9), 13921398 (2007).

28. Vansteenkiste JF, Stroobants SG, Dupont PJ, et al., Prognostic importance of the standardized uptake value on (18)F-fluoro-2-deoxy-glucose-positron emission tomography scan in non-small-cell lung cancer: An analysis of 125 cases. Leuven Lung Cancer Group, J Clin Oncol 17 (10), 3201-3206 (1999).

29. Mac Manus MP, Hicks RJ, Matthews JP, et al., Metabolic (FDG-PET) response after radical radiotherapy/chemoradiotherapy for non-small cell lung cancer correlates with patterns of failure, Lung Cancer 49 (1), 95-108 (2005).

30. Aerts HJ, Bosmans G, van Baardwijk AA, et al., Stability of 18F-deoxyglucose uptake locations within tumor during radiotherapy for NSCLC: a prospective study, Int J Radiat Oncol Biol Phys 71 (5), 1402-1407 (2008).

31. ICRU Report 50, Prescribing, Recording, and reporting Photon Beam Therapy. International Commission on Radiation Units and Measurements, 1993. 
32. Nijsten SM, Mijnheer BJ, Dekker AL, et al., Routine individualised patient dosimetry using electronic portal imaging devices, Radiother Oncol 83 (1), 65-75 (2007).

33. A'Hern RP, Sample size tables for exact single-stage phase II designs, Stat Med 20 (6), 859866 (2001).

34. Bentzen SM, Dose painting and theragnostic imaging: towards the prescription, planning and delivery of biologically targeted dose distributions in external beam radiation oncology, Cancer Treat Res 139, 41-62 (2008).

35. Sovik A, Malinen E, Skogmo HK, et al., Radiotherapy adapted to spatial and temporal variability in tumor hypoxia, Int J Radiat Oncol Biol Phys 68 (5), 1496-1504 (2007).

36. Petit SF, Aerts HJ, van Loon JG, et al., Metabolic control probability in tumour subvolumes or how to guide tumour dose redistribution in non-small cell lung cancer (NSCLC): an exploratory clinical study, Radiother Oncol 91 (3), 393-398 (2009).

37. Thorwarth D, Eschmann SM, Paulsen F, et al., Hypoxia dose painting by numbers: a planning study, Int J Radiat Oncol Biol Phys 68 (1), 291-300 (2007). 



\section{CHAPTER}

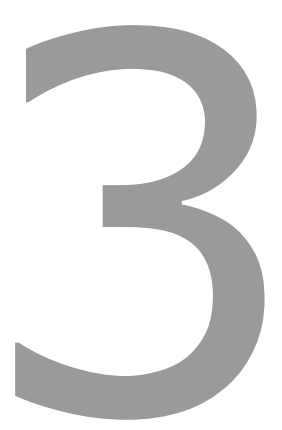

Identification of radioresistance:

Validation of location residual metabolic-active areas 


\section{ABSTRACT}

\section{Purpose}

It was recently described that on the basis of one pre-treatment 18F-deoxyglucose (FDG)-PET-CT scan, radio-resistant areas within lung tumors could be defined. To validate these results, we assessed the overlap-fraction (OF) of the high FDG-uptake areas pre-radiotherapy with the residual metabolic-active areas post-radiotherapy in an independent dataset.

\section{Methods and Materials}

Data from nineteen patients at Princess Margaret Hospital with lung cancer were analyzed. All patients underwent two FDG-PET-CT scans, one pre-radiotherapy (pre-RT) and one approximately three months post-RT. Sixteen patients showed residual FDGuptake after RT in the primary tumor. Residual FDG-uptake was defined as the FDGuptake within the primary tumor higher than the uptake within the aortic-arch. Seven of these 16 patients were excluded due to large deformations between the scans $(n=3)$ and because the residual disease was not clearly distinguishable from the surroundingtissue $(n=4)$. Therefore, 9 patients were included for further analysis. Autodelineations based on several threshold-levels relative to SUVmax were performed on the pre- and post-RT FDG-PET-CT scans within the primary-tumor. The volumes and OF's of these pre- and post-RT delineations were calculated.

\section{Results}

The mean volume of the residual metabolic-active areas was $20 \% \pm 18 \%$ (mean \pm SD) compared to the mean volume of the gross tumor volume (GTV) pre-RT. The residual metabolic-active areas largely corresponded with the pre-RT GTV (OF=88\% $\pm 15 \%)$. The pre-RT-scan threshold delineations of $34 \%, 40 \%$ and $50 \%$ of the SUVmax had a large OF with the residual region, $81 \% \pm 21 \%, 71 \% \pm 21 \%$ and $57 \% \pm 24 \%$, respectively. The mean volumes of the $34 \%, 40 \%$ and $50 \%$ uptake areas pre-RT were $58 \pm 47 \%, 43 \pm 31 \%$ and $26 \pm 20 \%$ of the GTV pre-RT.

\section{Conclusions}

In this independent dataset, we confirmed that the location of residual FDG-uptake areas after radiotherapy corresponds with the high FDG-uptake areas preradiotherapy. Therefore a pre-radiotherapy FDG-PET-CT scan can be used to identify the area that could be selectively boosted with a higher radiotherapy dose. 


\section{INTRODUCTION}

Lung cancer is still one of the most lethal solid tumors with a 5-year survival of about $20 \%$ in stage III non-small cell lung cancer (NSCLC) ${ }^{1,2}$. Although concurrent chemoradiation therapy improves local tumor control and long-term survival compared to sequential chemotherapy and radiotherapy (RT), local tumor failure is still observed in a significant proportion of stage III NSCLC patients ${ }^{2-5}$.

Local tumor control could be improved by radiation dose-escalation ${ }^{6-8}$. However, modeling studies suggest that at least 120 Gy would be required to achieve $90 \%$ local tumor control ${ }^{8}$. Delivering these high doses is presently not feasible because of doselimiting normal tissue toxicity. A possible solution would be to re-distribute the dose within the tumor, thus delivering higher doses to the most radio-resistant areas ${ }^{9-11}$. Recent research has shown that tumors are not homogeneous structures, but display significant intra-tumor variability in biological characteristics such as perfusion, hypoxia, cell density, and proliferation, which may result in areas of radio-resistance ${ }^{12-14}$.

Radiation dose re-distribution within the tumor is only possible when the radioresistant areas within the tumor can be determined. Since it has been shown that high uptake of F-deoxyglucose (FDG) in the primary tumor is associated with decreased survival and local control in NSCLC patients ${ }^{15-17}$, it is logical to hypothesize that the higher the FDG uptake, the more metabolically active and more radio-resistant it may be to radiotherapy. This was indeed shown in a recent study ${ }^{18}$. In the present study, we validated these findings in an independent group of patients that not only included NSCLC, but also small cell lung cancer (SCLC) patients.

In the present study, we assessed the overlap fraction (OF) of the pre-radiotherapy high FDG-uptake areas inside the primary lung tumor with the residual metabolicactive areas 3 months post-radiotherapy found on PET-CT scans. The purpose of this investigation is to validate the finding that high FDG uptake pre-treatment could be a good predictor to localize the resistant areas within the tumor.

\section{MATERIAL AND METHODS}

\section{Subjects}

Nineteen patients treated at Princess Margaret Hospital with lung cancer, 14 with NSCLC and 5 with SCLC with various stages were included. Patients were treated with concurrent chemo-RT ( $n=11)$, RT alone $(n=5)$ or sequential chemo-RT $(n=3)$. The mean age of the patient group was $67.4 \pm 11.6$ years (range: $43-80$ years). Patients included 
in the study were treated from February 2007 until December 2007. For each patient two FDG-PET-CT scans were available: the first scan was acquired before the start of RT, and the last approximately 3 months after the end of RT. Health Canada approved the use of FDG-PET-CT imaging in radiotherapy treatment planning and local Research Ethics Board approval was obtained for this research. The collected data were analyzed for the purpose of this retrospective study.

\section{Radiothrapy simulation}

Patients were simulated on a PET-CT-simulator with both arms above the head in an immobilization device. For the FDG-PET-CT scans a PET/CT Discovery ST-16 scanner (GE Healthcare, Milwaukee, USA) was used. After a minimum fasting time of 6 hours after which the blood glucose level was tested, $5 \mathrm{MBq} / \mathrm{Kg}$, up to a maximum of $500 \mathrm{MBq}$ of ${ }^{18}$ F-Fluoro-Deoxy-Glucose (FDG) was administered intravenously, followed by a $30 \mathrm{ml}$ saline flush. The patients were encouraged to rest during the uptake time of at least 60 minutes (mean pre-RT uptake time is 72 minutes, mean post-RT uptake time is 76 minutes), after which the PET-CT-scans were acquired. All patients also received a free breathing four-dimensional (4D) CT scan (Discovery ST, GE, Waukesha, WI) covering the complete thoracic region for planning purposes.

\section{Radiotherapy planning}

CT images corresponding to the exhale and inhale phases of respiration were transferred to the treatment planning system (Pinnacle, version 7.6c, Philips Radiation Oncology Systems, Milpitas, CA) using the exhale phase as the primary data set. This primary dataset was fused to the CT component of both PET-CT datasets using the rigidbody registration algorithm available in Syntegra. A physicist and physician prior to contouring assessed the quality of the registration.

Both the gross tumor volume (GTV) in the exhale phase and in the inhale phase were contoured on the 4D CT dataset by an experienced radiation oncologist, without using the initial FDG-PET data. These contours were copied onto the datasets containing registered PET-CT data and modified according to FDG uptake visualization. A $5 \mathrm{~mm}$ margin incorporating microscopic disease was added to the inhale and exhale GTV contours to generate the corresponding clinical target volume (CTV) contours. These inhale and exhale CTV contours were then fused to produce the internal target volume (ITV). A $5 \mathrm{~mm}$ uniform margin around the ITV determined the planning target volume (PTV). A 3D conformal plan for radiotherapy treatment was prepared and the individual dose was calculated according to the current departmental standards at Princess Margaret Hospital. 


\section{Image analysis}

The pre-RT and post-RT scans were used for image analysis, and was analyzed using the Pinnacle treatment planning system. The GTV contour, which was delineated on the exhale phase of the pre-RT 4D CT scan, was used for image analysis purposes. Additionally, high FDG uptake areas in the pre-RT scan within the primary tumor were contoured, using the thresholds $34 \%, 40 \%, 50 \%, 60 \%$, and $70 \%$ of the maximal SUV within the primary tumor. These thresholds correspond to the thresholds of the Dutch study ${ }^{18}$, in which the $34 \%$ threshold was found to correspond best to the GTV.

On the post-RT scan, residual disease was defined as an uptake within the location of the primary tumor higher than the Standardized Uptake Value (SUV) of the aortic arch in the same $\operatorname{scan}^{19}$. In addition, the commonly used fixed thresholds of SUV 2.5 and SUV 5.0 were delineated in the post-RT scan. Furthermore, for the post-RT scan, high FDG uptake areas using the thresholds $70 \%, 80 \%$ and $90 \%$ of the maximal SUV within the residual disease were contoured.

Pre-radiotherapy scan :

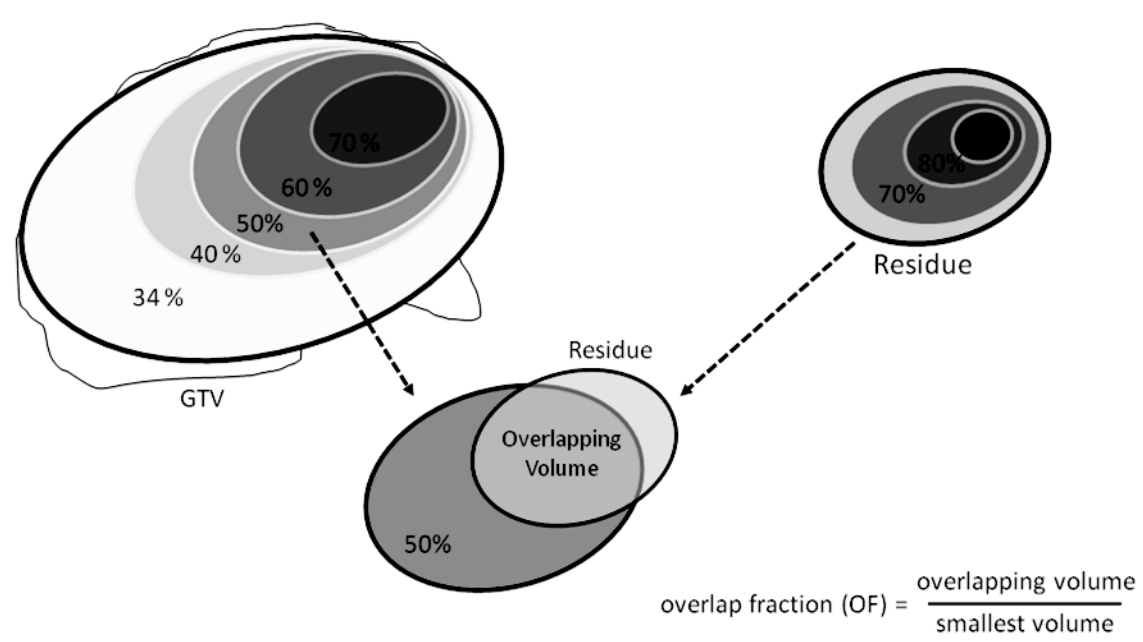

\section{Figure 1}

Schematic representations of delineations within the primary tumor on the pre-radiotherapy scan and within the residual disease on the post-radiotherapy scan. On the pre-scan the gross tumor volume (GTV) and the 34\%, 40\%, 50\%, 60\% and 70\% SUVmax FDG-PET uptake areas were delineated. On the post-scan the residual disease and the 70\%, 80\%, and 90\%SUVmax FDG-PET uptake areas within the residual uptake area were delineated. As an example, the overlapping volume and the Overlap Fraction (OF) of the 50\%SUVmax FDG high-uptake zone preradiotherapy with the residual uptake area are illustrated. 
The images of the post-RT scan were fused with those of the pre-RT scan using an automatic rigid registration algorithm. This automatic fusion was based on the bony anatomy (spine) at the level of the tumor, using a so-called clip box. If necessary, minor manual adjustments based on tissue surrounding the tumor were made to adjust the automatic fusion. After contouring on both scans, the overlap volume of all possible combinations of pre-RT and post-RT contours were determined using the Region Of Interest (ROI) expansion/contraction toolbox in Pinnacle. The volumes of these structures were also computed. Finally, the overlap fraction (OF) was calculated, defined as the volume of overlap divided by the smallest volume of the two delineations 18, 20 . A schematic representation of this method can be found in Figure 1. All data were expressed as mean \pm standard deviation (SD).

\begin{tabular}{|c|c|c|c|c|c|c|c|c|c|c|c|c|c|}
\hline 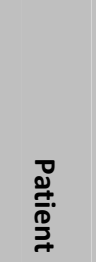 & 製 & 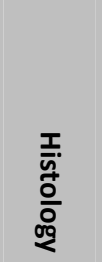 & 哭 & 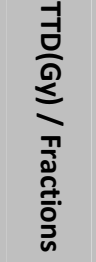 & 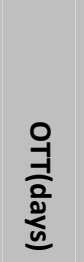 & 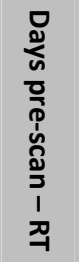 & 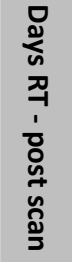 & $\begin{array}{l}\overrightarrow{7} \\
\bar{D} \\
\stackrel{2}{1} \\
\overrightarrow{3} \\
\mathbb{0} \\
2\end{array}$ & 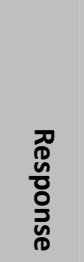 & 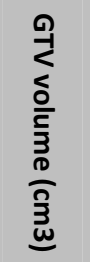 & 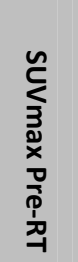 & 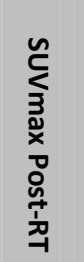 & 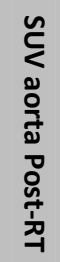 \\
\hline 1 & 78 & NSCLC & $\mathrm{T} 2 \mathrm{~N} 2 \mathrm{M} 0$ & $36 / 12$ & 15 & 18 & 56 & RT & SMD & 26.7 & 14.8 & 3.9 & 2.6 \\
\hline 2 & 62 & NSCLC & $\mathrm{T} 2 \mathrm{~N} 2 \mathrm{M} 0$ & $66 / 33$ & 30 & 35 & 83 & conc CT-RT & PMR & 95.5 & 28.3 & 10.6 & 3.6 \\
\hline 3 & 48 & NSCLC & T1N2M0 & $45 / 25$ & 36 & 7 & 42 & conc CT-RT & PMR & 5.7 & 5.2 & 7.7 & 3.3 \\
\hline 4 & 71 & NSCLC & T3N2M0 & $85 / 33$ & 45 & 19 & 62 & RT & PMR & 191.2 & 15.6 & 5.6 & 2.8 \\
\hline 5 & 79 & NSCLC & T1N2M1 & $36 / 12$ & 26 & 9 & 57 & RT & PMR & 7.5 & 10.9 & 6.8 & 3.2 \\
\hline 6 & 74 & NSCLC & T4NOMO & $66 / 33$ & 20 & 12 & 93 & conc CT-RT & PMR & 131.8 & 30.4 & 11.1 & 3.3 \\
\hline 7 & 59 & SCLC & Limited & $40 / 15$ & 25 & 9 & 114 & conc CT-RT & PMR & 25.9 & 10.9 & 4.3 & 3.2 \\
\hline 8 & 62 & NSCLC & T2NOMO & $36 / 12$ & 18 & 2 & 72 & seq RT-CT & PMR & 32.6 & 10.0 & 4.6 & 2.8 \\
\hline 9 & 56 & NSCLC & T2N2M1 & $36 / 12$ & 16 & 12 & 56 & seq RT-CT & SMD & 155.8 & 14.6 & 8.3 & 2.4 \\
\hline Mean: & 64.4 & & & & 25.6 & 13.6 & 70.6 & & & 74.7 & 15.6 & 7.0 & 3.0 \\
\hline Std: & 10.6 & & & & 10.0 & 9.6 & 22.5 & & & 70.3 & 8.4 & 2.7 & 0.4 \\
\hline $\min :$ & 56 & & & & 15 & 2 & 42 & & & 5.7 & 5.2 & 3.9 & 2.4 \\
\hline $\max :$ & 79 & & & & 45 & 35 & 114 & & & 191.2 & 30.4 & 11.1 & 3.6 \\
\hline
\end{tabular}

$N S C L C=$ non small cell lung cancer. $S C L C=$ small cell lung cancer. $R T=$ radiotherapy alone. Conc $C T-R T=$ Concurrent chemo-radiotherapy. Seq RT-CT = sequential radiotherapy and chemotherapy. $T T D=$ total tumor dose. $O T T=$ overall treatment time (only RT). GTV = Gross Tumor Volume. SUV = Standardized Uptake Value. 

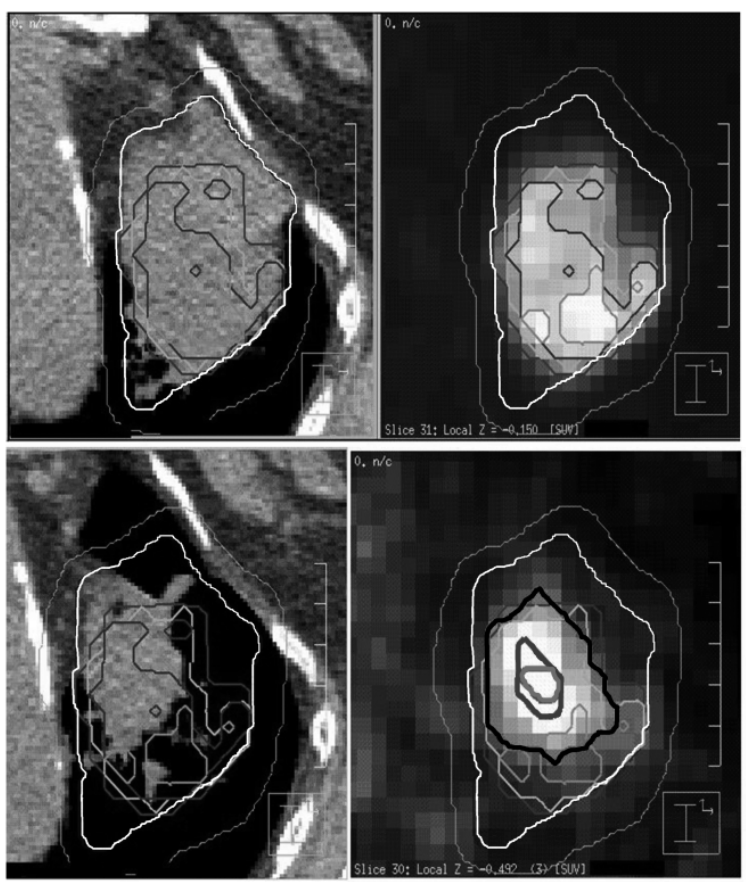

Figure 2

Representative images of a pre-RT FDG-PET-CT scan (upper) on a post-RT FDG-PET-CT scan (lower). The white line corresponds with the GTT. The thin gray lines correspond with the CTV and SUV thresholds in the pre-RT scan of 34\%,50\%,60\% and 70\%, respectively. The bold black line indicates the residual FDG-uptake higher than the aortic arch. The blod gray lines correspond with the SUV thresholds in the post-RT scan of 70 and $80 \%$, respectively.

\section{RESULTS}

\section{Patient Characteristics}

Ten of the nineteen patients were excluded from analysis. Firstly, patients who did not show residual disease in the last scan were excluded. Three patients were excluded for that reason: two of them were excluded because the residual uptake was lower than the SUV of the aortic arch and the third only showed residual uptake in the lymph nodes. Secondly, another four patients were excluded because the tumor was not definable from the surrounding tissue on the post-RT scan. In two cases this was caused by radiation pneumonitis. In the other two cases it was impossible to distinguish between the tumor and the heart or great vessels due to the location of the tumor. Last 


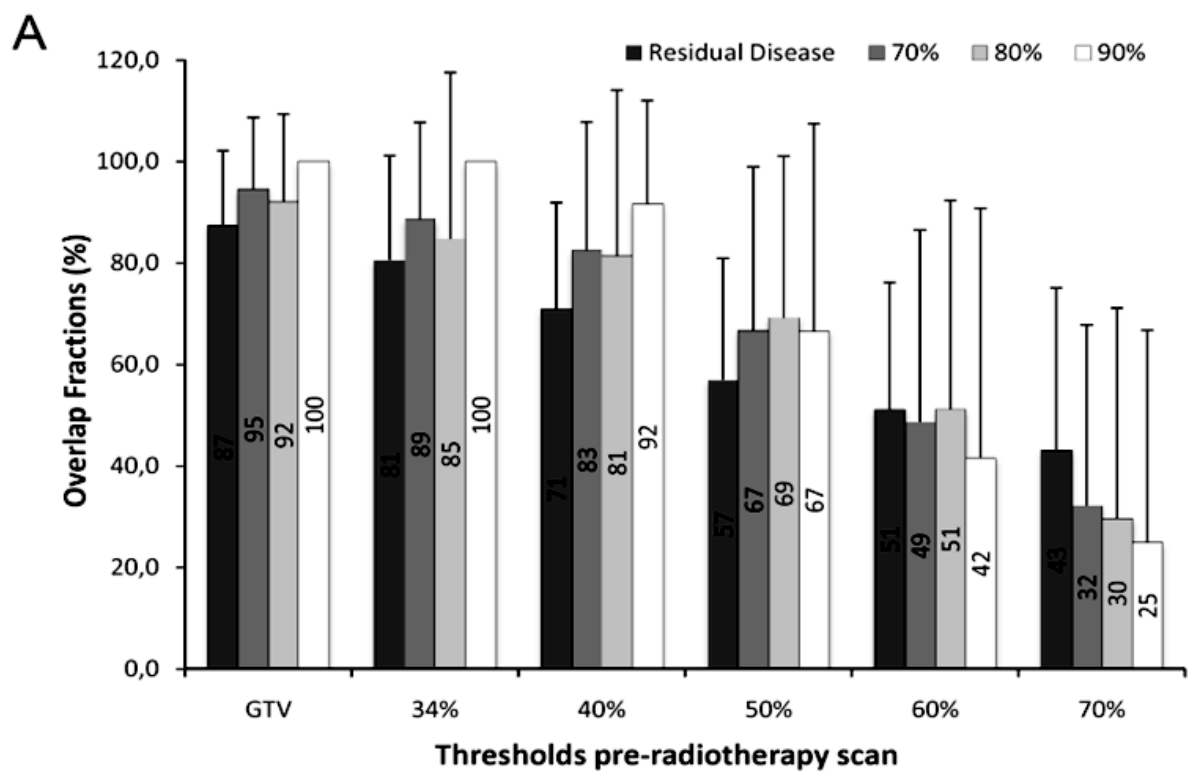

B

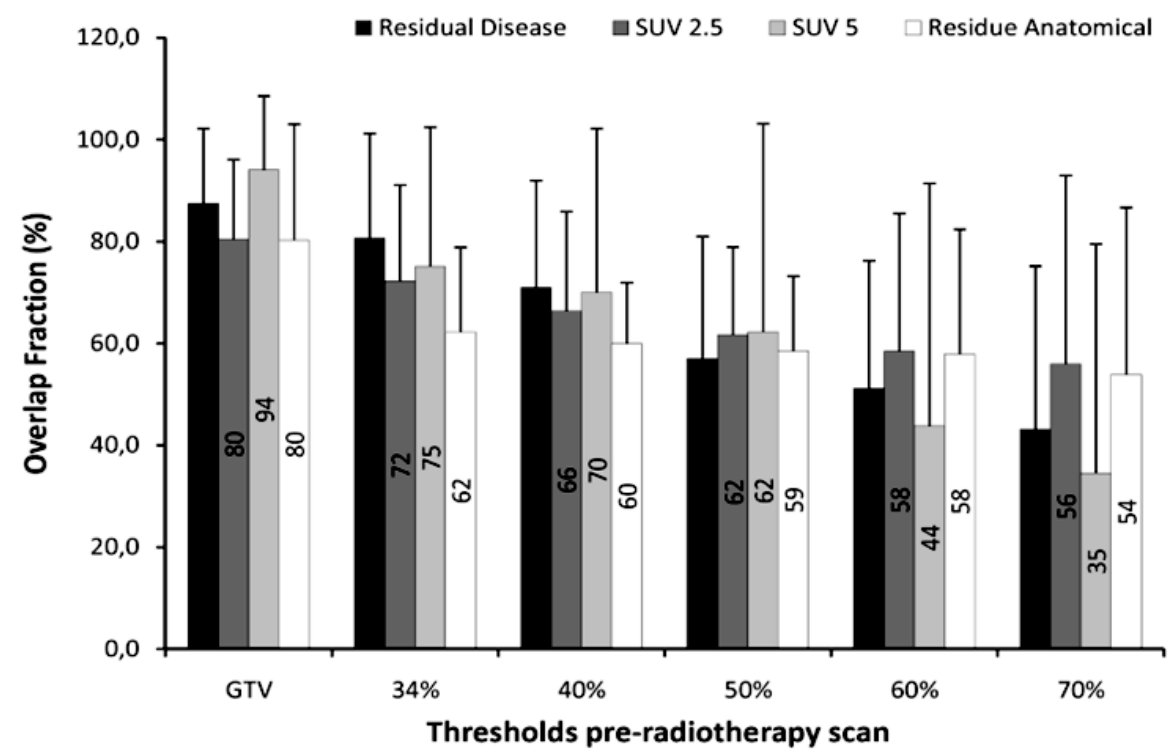

Figure 3

Overlap fractions (OF) of the pre-radiotherapy with the post-radiotherapy relative (A) and absolute (B) SUVmax thresholds are shown. OF's of the post-radiotherapy residual-areas with a higher uptake than the aortic arch are shown in both figures. All data were expressed as mean \pm standard deviation (SD). 
ly, three patients were excluded due to large tumor deformations. Therefore the analysis was performed on nine patients. Additional information about the patients included in this study can be found in Table 1.

\section{Overlap fractions}

Figure 2 shows the pre and post-RT FDG-PET-CT scan of a representative patient with several pre-RT and post-RT delineations. It can be clearly seen, that the residual metabolic area appears at the high FDG uptake zone of the pre-RT scan. The residual areas were mainly located within the original GTV (OF $=87.5 \% \pm 14.7 \%$ ). This was also the case for the $70 \%$ and $80 \%$ SUVmax thresholds. The OF of these thresholds with the GTV were $94.6 \% \pm 14.2 \%$ and $92.2 \% \pm 17.2 \%$, respectively. The $90 \%$ SUVmax was $100 \%$ located within the GTV. The pre-RT-scan threshold delineations of $34 \%, 40 \%$ and $50 \%$ of the SUVmax had a large OF with the residual regions of $81 \% \pm 21 \%, 71 \% \pm 21 \%$ and $57 \% \pm 24 \%$, respectively. The overlap fractions are shown in Figures $3 \mathrm{a}$ and $3 \mathrm{~b}$.

\section{Threhold volumes}

The relative volumes of the delineated areas on the pre-RT scan are shown in Figure 4a. It can be clearly seen that the automatically contoured high FDG uptake areas were small compared to the GTV volume: $58.1 \% \pm 46.6 \%$ for the $34 \%$-threshold; $25.5 \% \pm 19.7 \%$ for the $50 \%$-threshold and only $6.1 \% \pm 7.6 \%$ for the $70 \%$ threshold.

The relative volumes of the delineated areas on the post-RT scan are shown in Figure 4b. Here it can also be clearly seen that the automatically contoured high FDG uptake areas were small compared to the GTV volume: $20.2 \% \pm 17.8 \%$ for the residual disease based on the uptake higher than the aortic arch and $2.5 \% \pm 2.0 \%$ for the threshold of $70 \%$. The areas contoured by the absolute thresholds of SUV 2.5 and 5.0 were also quite small: $33.6 \% \pm 22.2 \%$ resp. $2.6 \% \pm 3.5 \%$. 

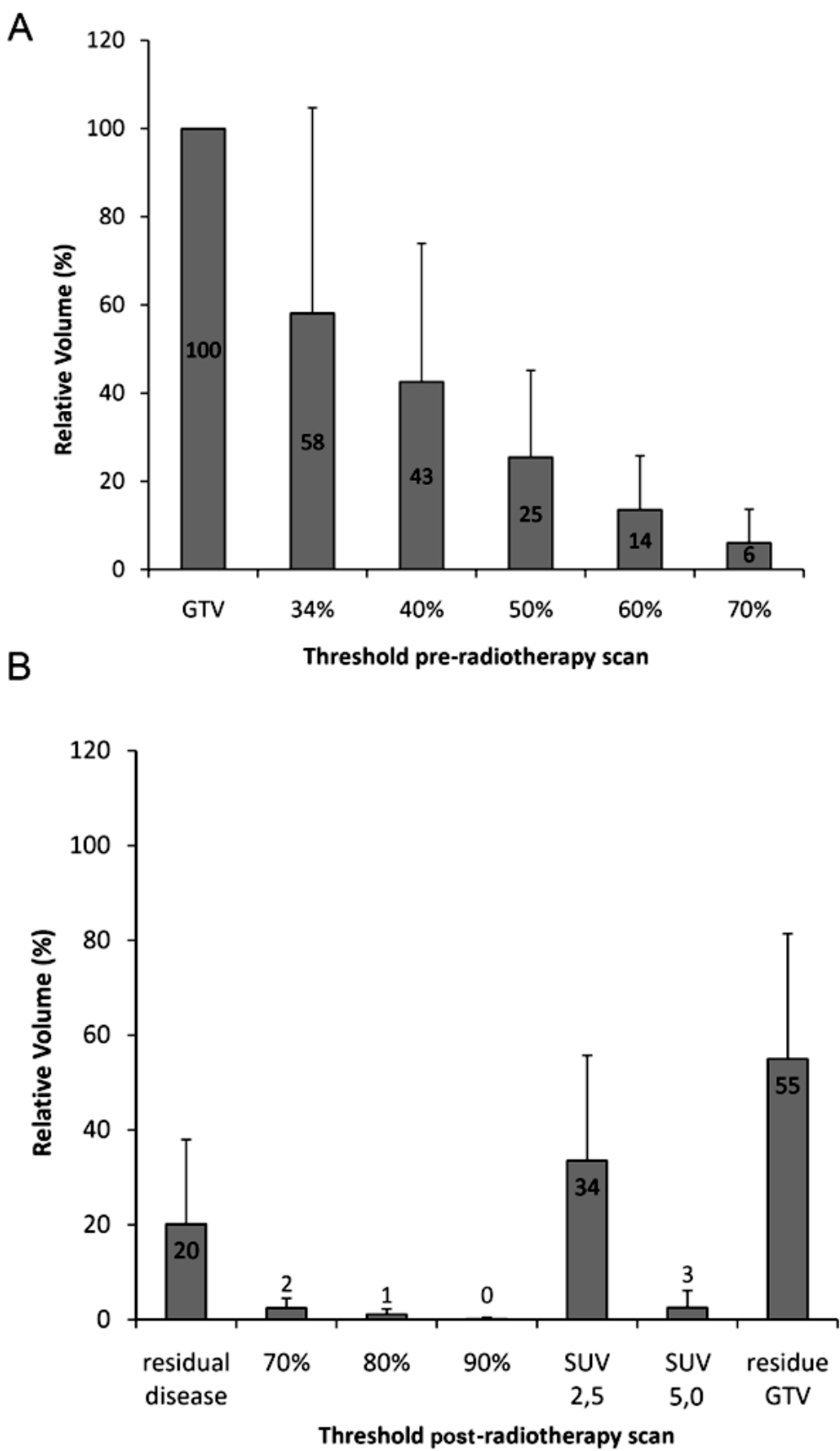

Figure 4

Volumes of the SUV thresholds of the tumor pre-radiotherapy (A) and post-radiotherapy (B). All volumes ( $A$ and $B$ ) are relative to the pre-radiotherapy gross tumor volume (GTV). All data were expressed as mean \pm standard deviation (SD). 


\section{DISCUSSION}

The identification of radio-resistant areas within the tumor would be of great importance for it is a prerequisite for redistribution of the dose in the tumor, which could improve the outcome in lung cancer patients treated with radiotherapy $10,21,22$. Recently, it was found that the high-uptake areas of FDG in the tumor before therapy identify radiation-resistant zones 18 . These areas remain at the same position during treatment and are identifiable on the pre-treatment FDG-PET-CT scan and are thus suitable for selective dose re-distribution within the tumor 9, 20. Moreover, a clear relationship between the SUV of the FDG uptake in the tumor and the tumor control probability has been described 23 . The results were nevertheless never validated in an independent data set.

In the present study, residual metabolic activity after treatment was found in the areas that were most FDG-avid before therapy. The residual FDG uptake areas largely corresponded with the $40 \%$ SUV uptake zone on the pre-RT scan, resulting in an overlap fraction of $71 \%$. The $40 \%$ SUV high uptake area corresponds to $43 \%$ of the volume of the original tumor volume, which is comparable with the results in MAASTRO 18 . The residual areas were located almost completely (OF $=87 \%$ ) within the gross tumor volume, and always within the planning target volume, indicating no geographical miss. Also, the hotspot within the residual areas (90\% SUVmax) was completely within the GTV (OF=100\%). It has to be noticed that the absolute values of the thresholds of the high-uptake areas and the volumes differ in the present series from the Dutch publication ${ }^{18}$. This may be due to the PET protocol that differed substantially, the inclusion of a variety of tumor stages and treatments, the smaller number of patients and the inclusion of both NSCLC and SCLC. This underscores the necessity for thorough standardization before multi-center studies can be launched 24 .

The differences in volumes and standard deviations between the patient groups of MAASTRO and PMH may be caused by the patient groups: In this study both NSCLC and SCLC patients are included, in the Dutch group only NSCLC patients. A limitation of the present study is the large proportion of excluded patients due to deformation and high uptake in surrounding tissues, which made it impossible to delineate the tumor properly. However, to the best of our knowledge, no validated methods have been designed to solve the problem of registration of images with time intervals of weeks.

Nevertheless, this validation study shows again that a single pre-RT FDG-PET-CT scan can identify the areas within the tumor that contain cells that will show persistent FDG uptake post-RT, thus pointing to defined areas in the primary tumor that might be beneficial for boosting. 


\section{REFERENCES}

1. Jemal A, Siegel R, Ward E, et al., Cancer statistics, 2008, CA Cancer J Clin 58 (2), 71-96 (2008).

2. Rowell NP and O'Rourke N P, Concurrent chemoradiotherapy in non-small cell lung cancer, Cochrane Database Syst Rev (4), CD002140 (2004).

3. Garrido $P$, Gonzalez-Larriba JL, Insa A, et al., Long-term survival associated with complete resection after induction chemotherapy in stage IIIA (N2) and IIIB (T4NO-1) non small-cell lung cancer patients: the Spanish Lung Cancer Group Trial 9901, J Clin Oncol 25 (30), 47364742 (2007).

4. Betticher DC, Hsu Schmitz SF, Totsch M, et al., Prognostic factors affecting long-term outcomes in patients with resected stage IIIA pN2 non-small-cell lung cancer: 5-year follow-up of a phase II study, Br J Cancer 94 (8), 1099-1106 (2006).

5. Furuse K, Fukuoka M, Kawahara M, et al., Phase III study of concurrent versus sequential thoracic radiotherapy in combination with mitomycin, vindesine, and cisplatin in unresectable stage III non-small-cell lung cancer, J Clin Oncol 17 (9), 2692-2699 (1999).

6. Bradley J, A review of radiation dose escalation trials for non-small cell lung cancer within the Radiation Therapy Oncology Group, Semin Oncol 32 (2 Suppl 3), S111-113 (2005).

7. Hayman JA, Martel MK, Ten Haken RK, et al., Dose escalation in non-small-cell lung cancer using three-dimensional conformal radiation therapy: update of a phase I trial, J Clin Oncol 19 (1), 127-136 (2001).

8. Fowler JF, Tome WA, Fenwick JD, et al., A challenge to traditional radiation oncology, Int J Radiat Oncol Biol Phys 60 (4), 1241-1256 (2004).

9. Kim $Y$ and Tome WA, Is it beneficial to selectively boost high-risk tumor subvolumes? A comparison of selectively boosting high-risk tumor subvolumes versus homogeneous dose escalation of the entire tumor based on equivalent EUD plans, Acta Oncol 47 (5), 906-916 (2008).

10. Bentzen SM, Theragnostic imaging for radiation oncology: dose-painting by numbers, Lancet Oncol 6 (2), 112-117 (2005).

11. Tanderup K, Olsen DR and Grau C, Dose painting: art or science?, Radiother Oncol 79 (3), 245-248 (2006).

12. Cooper RA, Carrington BM, Loncaster JA, et al., Tumour oxygenation levels correlate with dynamic contrast-enhanced magnetic resonance imaging parameters in carcinoma of the cervix, Radiother Oncol 57 (1), 53-59 (2000).

13. Vanderstraeten B, Duthoy W, De Gersem W, et al., [18F]fluoro-deoxy-glucose positron emission tomography ([18F]FDG-PET) voxel intensity-based intensity-modulated radiation therapy (IMRT) for head and neck cancer, Radiother Oncol 79 (3), 249-258 (2006).

14. Piert $M$, Machulla HJ, Picchio $M$, et al., Hypoxia-specific tumor imaging with $18 \mathrm{~F}$ fluoroazomycin arabinoside, J Nucl Med 46 (1), 106-113 (2005).

15. Eschmann SM, Friedel G, Paulsen F, et al., Is standardised (18)F-FDG uptake value an outcome predictor in patients with stage III non-small cell lung cancer?, Eur J Nucl Med Mol Imaging 33 (3), 263-269 (2006).

16. Borst GR, Belderbos JS, Boellaard R, et al., Standardised FDG uptake: a prognostic factor for inoperable non-small cell lung cancer, Eur J Cancer 41 (11), 1533-1541 (2005). 
17. Downey RJ, Akhurst T, Gonen M, et al., Preoperative F-18 fluorodeoxyglucose-positron emission tomography maximal standardized uptake value predicts survival after lung cancer resection, J Clin Oncol 22 (16), 3255-3260 (2004).

18. Aerts HJ, van Baardwijk AA, Petit SF, et al., Identification of residual metabolic-active areas within individual NSCLC tumours using a pre-radiotherapy (18)Fluorodeoxyglucose-PET-CT scan, Radiother Oncol 91 (3), 386-392 (2009).

19. Mac Manus MP, Hicks RJ, Matthews JP, et al., Metabolic (FDG-PET) response after radical radiotherapy/chemoradiotherapy for non-small cell lung cancer correlates with patterns of failure, Lung Cancer 49 (1), 95-108 (2005).

20. Aerts HJ, Bosmans G, van Baardwijk AA, et al., Stability of 18F-deoxyglucose uptake locations within tumor during radiotherapy for NSCLC: a prospective study, Int J Radiat Oncol Biol Phys 71 (5), 1402-1407 (2008).

21. Bentzen SM, Dose painting and theragnostic imaging: towards the prescription, planning and delivery of biologically targeted dose distributions in external beam radiation oncology, Cancer Treat Res 139, 41-62 (2008).

22. Sovik A, Malinen E, Skogmo HK, et al., Radiotherapy adapted to spatial and temporal variability in tumor hypoxia, Int J Radiat Oncol Biol Phys 68 (5), 1496-1504 (2007).

23. Petit SF, Aerts HJ, van Loon JG, et al., Metabolic control probability in tumour subvolumes or how to guide tumour dose redistribution in non-small cell lung cancer (NSCLC): an exploratory clinical study, Radiother Oncol 91 (3), 393-398 (2009).

24. Boellaard R, Standards for PET image acquisition and quantitative data analysis, J Nucl Med 50 Suppl 1, 11S-20S (2009). 



\section{CHAPTER}

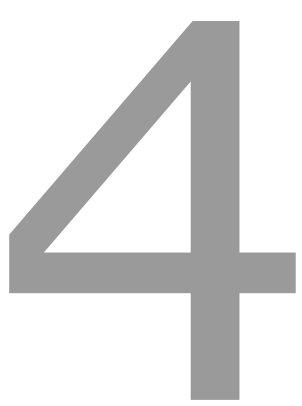

Identification of radioresistance:

Prospective validation of residual activity

Identification of Residual Metabolic-active Areas within NSCLC Tumours using a PreRadiotherapy FDG-PET-CT Scan: A prospective validation

Hugo J.W.L. Aerts, Johan Bussink, Wim J.G. Oyen, Wouter van Elmpt, Annemieke M. Folgering, Daisy Emans, Marije Velders, Philippe Lambin, Dirk De Ruysscher 


\section{SUMMARY}

It was recently described that high FDG-uptake areas pre-radiotherapy largely correspond with residual metabolic-active areas post-radiotherapy. Here, an independent prospective validation confirmed these results using an overlap-fraction calculation of various FDG-uptake based thresholds. These results show that high FDG-uptake preradiotherapy represent a potential target for radiation boosting.

\section{ACKNOWLEDGEMENTS}

The authors acknowledge financial support from the Dutch Cancer Society (KWF: fellowship granted to H.J.W.L.A.), as well as technical and financial support from Siemens MI. 


\section{INTRODUCTION}

Lung cancer is one of the most frequent and lethal solid tumours ${ }^{1}$. Although the prognosis has improved, the 5 -year survival is about $20 \%$ in locally advanced non-small cell lung cancer (NSCLC) ${ }^{2,3}$. While concurrent chemo-radiation improved local control and long-term survival compared to traditional sequential (chemo-) radiotherapy, local tumour failure is still observed in the majority of patients ${ }^{4-6}$. Improvements in local tumour control can be achieved by escalating the radiation dose, where local control rates over $90 \%$ can be reached for tumour doses of $120 \mathrm{~Gy}^{7,8}$. However, the problem is that tumour dose escalation is limited by the radiation toxicity of normal tissues such as the lungs and the spinal cord.

A solution to this problem may be found in the intra-tumour heterogeneity. It has become increasingly clear that tumour tissue is often not homogeneous, but can have large spatial variations with different biological characteristics, like perfusion, hypoxia, cell density, proliferation and subsequent radio-resistance ${ }^{9-14}$. Therefore, a strategy to increase the dose to more radio-resistant areas within the tumour, while reducing the dose to more susceptible zones, can result in better local control rates with the same healthy tissue toxicity ${ }^{15}$.

A strategy to determine the location of the radio-resistant areas within the tumour is the use of non-invasive imaging. Since high pre-treatment ${ }^{18} \mathrm{~F}$-deoxyglucose (FDG) uptake levels in the primary tumour result in lower survival in patients with NSCLC ${ }^{16-18}$, we hypothesized that the regions with high FDG uptake before treatment identify more radio-resistant areas within the tumour. If true, an increased survival could be expected from radiation dose redistribution according to FDG uptake preradiotherapy. In an earlier study, we demonstrated that this hypothesis holds true for a group of NSCLC patients in MAASTRO Clinic (Maastricht, The Netherlands) ${ }^{19}$. Here, we report on an independent prospective dataset to validate that the high FDG uptake areas pre-radiotherapy identify the residual metabolic active areas post-radiotherapy.

\section{MATERIAL AND METHODS}

\section{Patient Characteristics}

Twelve patients of Radboud University Nijmegen Medical Center (Nijmegen, The Netherlands) with inoperable non-small cell lung cancer (NSCLC), UICC stage II-III, eleven treated with sequential chemo-radiotherapy and one with radical radiotherapy (RT) alone. The sequential chemo-radiotherapy schedule consisted of 1 to 3 cycles of cisplatin and gemcitabine before the start of RT. Patients were included from June 2007 
until August 2008. Mean age was 66.2 \pm 9.5 years (range: $52-83$ years).Two FDG-PET-CT scans were available for each patient. The first scan was performed before start of RT while the second scan was performed approximately three months after the end of RT.For the patients receiving sequential chemo-radiotherapy was the pre-RT scan performed after the chemotherapy. No treatment was given to any of the patients between the end of RT and the post-RT scan.

\section{Radiotherapy Simulation and Planning}

Patients were simulated in radiotherapy position on a CT-simulator, multislice spiral CT scanner (Philips AcQsim, Philips, Cleveland, USA) with both arms above the head. For the FDG-PET-CT scans a Siemens Biograph (Siemens/CTI, Knoxville, Tennessee, USA) was used. An intravenous injection of $250 \mathrm{MBq}$ FDG (Covidien, Petten, The Netherlands)was followed by $10 \mathrm{ml}$ normal saline. After a 60 minutes uptake period, during which the patient was encouraged to rest, PET-CT images were acquired.

Radiotherapy planning was performed on a Pinnacle (version 8.0d; Philips Radiation Oncology System, Madison, WI, USA), the radiotherapy planning system routinely used which is based on a convolution algorithm using inhomogeneity corrections. The Gross Tumour Volume (GTV) and the Planning Target Volume (PTV) were defined for all patients, based on PET and CT data. The Clinical Target Volume (CTV) was defined as the GTV with a $5 \mathrm{~mm}$ margin incorporating microscopic disease. Subsequently, this CTV was expanded with $1 \mathrm{~cm}$ to draw the PTV to incorporate the internal respiratory motion and setup errors. A 3D conformal treatment plan was calculated on the PTV for all patients according to ICRU 50 guidelines. Dosimetric values were calculated on the basis of dose-volume histograms and dose distributions on each axial CT plan.

\section{Image Analysis}

The pre- and post-RT scans were analyzed and delineated using the Siemens TrueD system (Version VC-30, Siemens A.G., Darmstad, Germany). On the pre-RT scan, the location and volume of the FDG uptake within the GTV were quantified using the threshold $34,40,50,60$ and $70 \%$ of the maximal SUV $\left(S U V_{\max }\right)$. On the post-RT scan, residual metabolic areas were defined as FDG uptake higher than in the aortic arch $\left(S U V>S U V_{\text {aorta }}\right)^{20}$. Within the residual FDG-positive areas, the high FDG uptake areas were defined using the thresholds 70,80 , and $90 \%$ of the tumour SUV $_{\max }$. Using an automatic rigid-registration algorithm, based on mutual information of the CT scans, the images of pre-RT scan were fused to the post-RT scan on the Siemens TrueD system. If the automatic registration showed a large deviation between the two CT scans, the images were manually registered on the anatomy surrounding the tumour, e.g. the bony anatomy or large vessels. The contours of the FDG-based thresholds on the postRT scan were then transformed to the pre-RT scan using the derived registration matrices. The contours of the pre- and post-RT scan were exported from TrueD as DICOM- 
RT structure sets. Using MATLAB (R2008b, The MathWorks Inc, Natick, MA, USA) the overlap fractions (OF) and volumes of these contours were calculated. The overlap fraction was defined as the volume of overlap divided by the smallest volume ${ }^{19,21}$. By using this methodology it is possible to assess which threshold on the pre-RT scan matches the residual disease on the post-RT scan. All data are expressed as mean \pm standard deviation (SD) and range.

\section{RESULTS}

\section{Patient characteristics}

To assess whether the location of the residual metabolic active areas are located within the initial FDG-high uptake areas of the primary tumour, two ${ }^{18}$ Fluorodeoxyglucose (FDG) PET-CT scans were analyzed for all patients, one before radiotherapy (pre-RT) and one after radiotherapy (post-RT). Of the twelve analyzed patients, four patients showed no residual FDG uptake on the post-RT scan and had a complete metabolic response. The other eight patients showed residual FDG uptake within the primary tumour $\left(S_{U} V_{\max }>S U V_{\text {aorta }}\right)$. One of these patients had a residue that was not clearly distinguishable from the surrounding tissue due to FDG avid inflammation. Therefore, seven patients remained for further analysis.

\section{Overlap of FDG-uptake before RT with residual areas}

In Figure 1, pre- and post-RT CT-PET images are shown of a representative patient. The high FDG uptake areas $\left(50 \%\right.$ SUV $\left._{\text {max }}\right)$ on the pre-RT scan and the location of the residual areas on the post-RT scan are shown. The residual areas are transposed to the preRT scan, to show the overlap with $50 \%$ SUV $_{\max }$ high uptake area pre-RT. Visual evaluation shows that the location of the residual areas largely corresponds with the high FDG uptake areas pre-RT.

\section{Overlap fractions of the FDG based thresholds}

Figure $2 \mathrm{~A}$ depicts the overlap fractions and volumes of the FDG uptake based thresholds within the primary tumour pre-RT and post-RT. Here it is shown that the residual areas were mainly located within the original GTV (OF=93.7 $\pm 7.2 \%$ [range: $82.8-100$ ]). Also, the $70 \%, 80 \%$ and $90 \%$ SUV $_{\max }$ high-uptake areas within the residual areas showed a high overlap with the GTV volume (OF $=93.5 \pm 10.6 \%$ [range: $71.6-100 \%$ ], $94.2 \pm 11.5 \%$ [range: $69.6-100.0 \%$ ], and $96.1 \pm 10.2 \%$ [range: $73.0-100 \%$ ] respectively). 

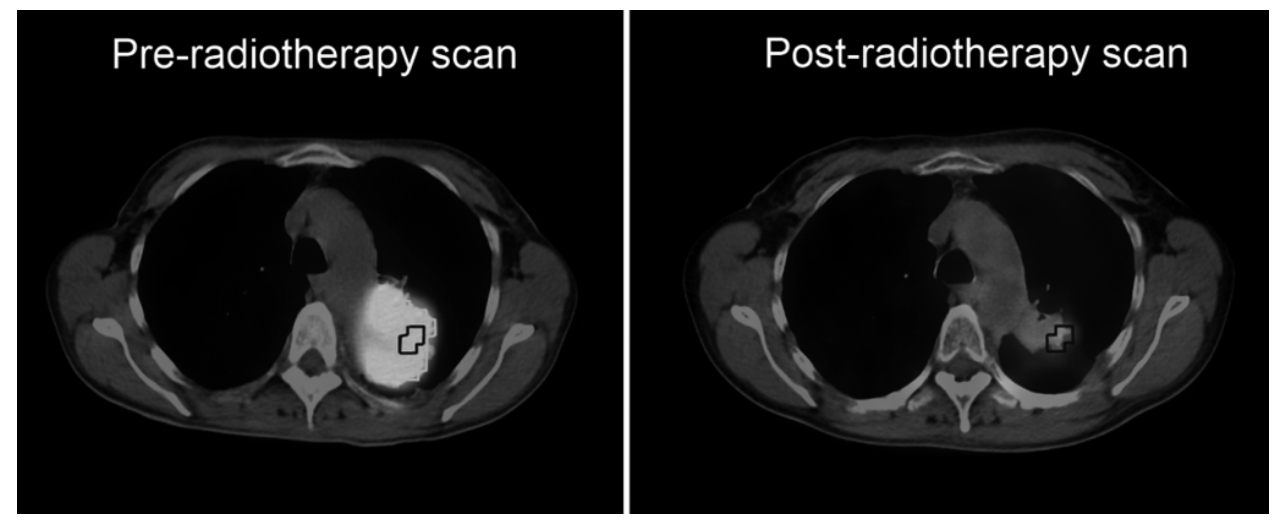

\section{Figure 1}

Representative FDG-PET-CT images of a patient pre- and post-radiotherapy. The light line indicate the 50\%SUV $\max$ FDG high uptake area pre-radiotherapy. The dark lines indicate the residual metabolic active areas post-radiotherapy, also transposed on the pre-radiotherapy scan. Visual evaluation shows a large correspondence between the residual areas post-radiotherapy with the high FDG uptake areas pre-radiotherapy.

Also, the pre-RT FDG uptake thresholds had a large overlap with the residual areas. The 34\% threshold pre-RT had an OF with the residual areas of $86.9 \pm 8.3 \%$ [range: 77.2 $98.2 \%]$ and with the high-uptake areas within the residue. Moreover, the same is true for the pre-RT $40 \%$ and $50 \%$ SUV $_{\max }$ high FDG uptake area (OF $=77.4 \pm 8.1 \%$ [range: $67.7-89.9 \%$ ] and OF $=67.9 \pm 6.8 \%$ [range: $61.5-82.6 \%$ ]). This pre-RT 50\% SUV $\max$ area also largely corresponded with the $70-90 \%$ SUV $_{\max }$ high-uptake areas within the residue.

\section{Volumes of the FDG based thresholds}

The volumes of the FDG based thresholds within the primary tumour pre-RT and the post-RT thresholds are shown in Figure 2B. The FDG uptake areas $\left(40-70 \% \mathrm{SUV}_{\max }\right)$ within the tumour on the pre-RT scan were small compared to the GTV. The $40 \%$ SUVmax encompassed $46.4 \pm 14.4 \%$ range: [23.0-66.5\%] of the original GTV, whereas this was $35.8 \pm 11.8 \%$ [range: $19.6-54.7 \%$ ] for the $50 \%$ SUVmax, $23.9 \pm 7.8 \%$ [range: 13.2 $37.5 \%$ ] for the $60 \%$ SUVmax, and $12.9 \pm 5.1 \%$ [range: $8.2-23.3 \%$ ] for the $70 \%$ SUVmax threshold.

The volume of the residual metabolic active areas on the post-RT scan was $14.6 \pm 10.0 \%$ [range: 4.3-31.2\%] of the pre-RT GTV (Fig. 2B). The relative volumes of the high uptake areas within the residual areas were very small: $11.9 \pm 7.7 \%$ [range: $2.9-15.8 \%$ ] for the $70 \%$ SUV $_{\max }, 5.8 \pm 4.9 \%$ [range: $0.8-15.6 \%$ ] for the $80 \% \mathrm{SUV}_{\max }$, and $1.9 \pm 2.0 \%$ [range: 0.3-6.1\%] for the $90 \%$ SUV $_{\max }$ threshold. 

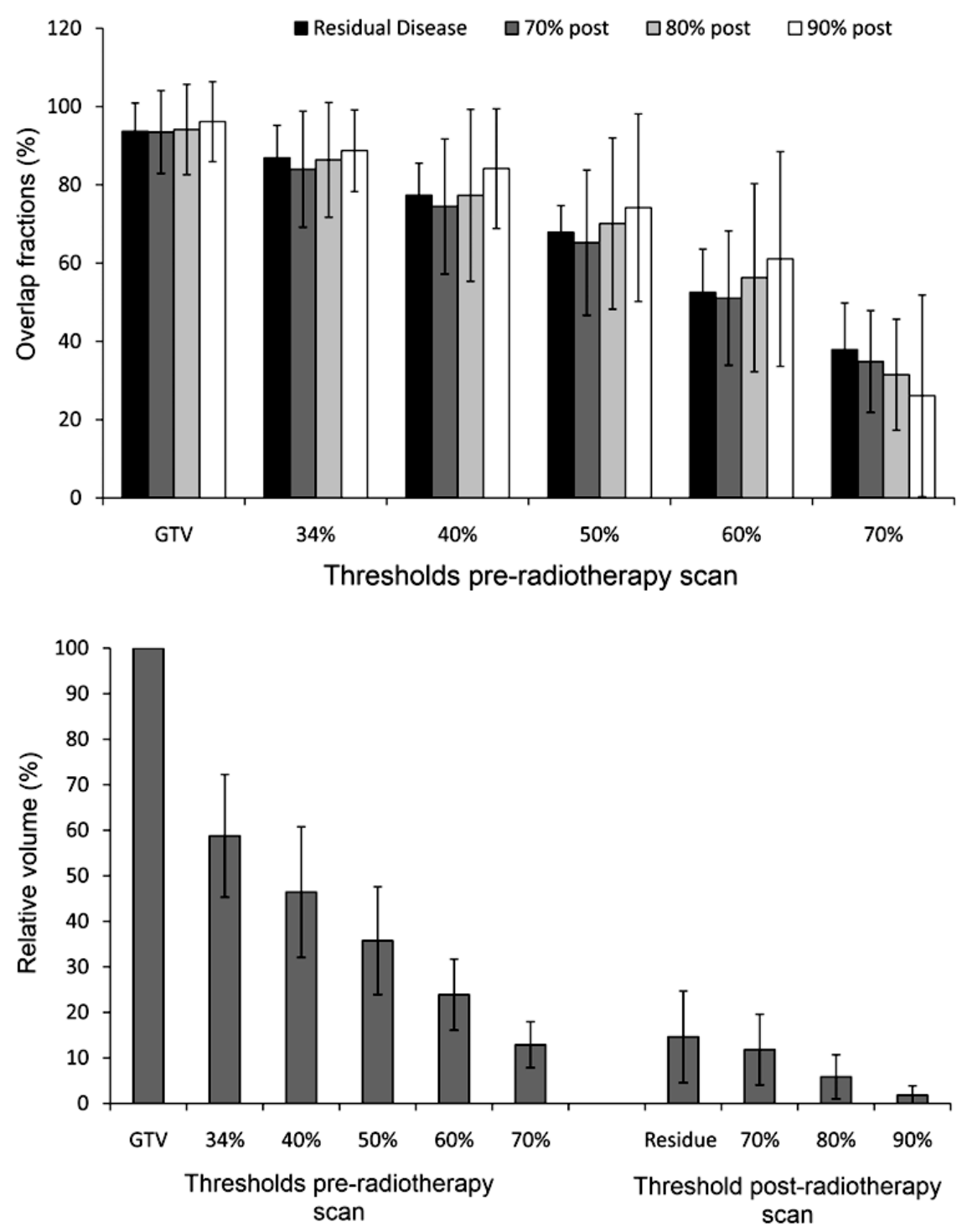

\section{Figure 2}

A: Overlap-fractions (OF) of the pre-radiotherapy thresholds with the post-radiotherapy relative thresholds. The OF of the post-radiotherapy residual-areas are indicated with the black-bars. The other bars indicate the OF with the high FDG-uptake (70-90\%) areas within the residue. The data are expressed as mean \pm standard deviation (error-bars). B: Volumes of the SUV thresholds of the tumour pre- and post-radiotherapy. All volumes are relative to the pre-radiotherapy gross tumour volume (GTV). The data are expressed as mean \pm standard deviation (error-bars). 


\section{DISCUSSION}

In radiation oncology there is an growing interest to increase the radiation dose towards more resistant tumour areas whilst reducing the dose to less resistant areas within the tumour ${ }^{15}$. In the present study, we confirmed in a prospective study the results that the areas of high FDG uptake within the tumour before treatment would allow identification of residual metabolic-areas after therapy ${ }^{19}$. Together with the results of the previous study, where it was demonstrated that the high-uptake areas of FDG within the tumour remained stable during a course of fractionated radiotherapy for NSCLC ${ }^{21}$, radiation boosting of these zones might be beneficial.

Here, we have shown that the residual FDG uptake areas after treatment largely corresponded to the $50 \% \mathrm{SUV}_{\max }$ within the tumour before treatment, resulting in an overlap fraction of $68 \%$. This was similar with the previous obtained results ${ }^{19}$, where the overlap between these contours was $71 \%$. The volume of the residual FDG-positive areas was on average $15 \%$ of the initial GTV volume. The hotspot within the residual area (90\%SUV) was almost completely within the pre-RT GTV (OF=96\%), and had a high overlap with the pre-radiotherapy $50 \%$ SUV threshold (OF>74\%). Based on these results, we conclude that residual metabolic active areas within the tumour can be identified before treatment using a FDG-PET-CT scan.

The pre-radiotherapy $50 \%$ SUV $_{\max }$ threshold had a volume that encompassed $35 \%$ of pre-treatment GTV, similar with previous results (39\%). This makes the $50 \% \mathrm{SUV}_{\max }$ threshold value a suitable threshold for future radiation boosting target to improve local control, not only because it yields good results, but also because it is a simple, reproducible, and robust method between institutions. A lower threshold would result in boosting the entire tumour and not only the most resistant areas, whereas a too high threshold would lead to only a few voxels to be treated, which is often impossible due to organ motion or dose delivery constraints.

A limitation of our study is that for the registration between the pre and post-scans a rigid registration was performed, not incorporating the non-rigid tissue changes possibly induced by radiotherapy. This could be improved by using deformable registration techniques. However, these are difficult to validate and the reproducibility, especially in different institutes, is limited.

In conclusion, our previous results were prospectively validated in an independent dataset in another institute, showing that the residual metabolic-active areas within the tumour after (sequential chemo-) radiotherapy are located in the high FDG uptake areas before therapy and can be delineated. This information is the basis for an ongoing randomized phase II trial to test the hypothesis that high FDG uptake areas reflect 
"radioresistance", by redistribution the dose according to pre-radiotherapy FDG uptake areas.

\section{REFERENCES}

1. Jemal A, Siegel R, Ward E, et al., Cancer statistics, 2008, CA Cancer J Clin 58 (2), 71-96 (2008).

2. Betticher DC, Hsu Schmitz SF, Totsch M, et al., Prognostic factors affecting long-term outcomes in patients with resected stage IIIA pN2 non-small-cell lung cancer: 5-year follow-up of a phase II study, Br J Cancer 94 (8), 1099-1106 (2006).

3. van Meerbeeck JP, Kramer GW, Van Schil PE, et al., Randomized controlled trial of resection versus radiotherapy after induction chemotherapy in stage IIIA-N2 non-small-cell lung cancer, J Natl Cancer Inst 99 (6), 442-450 (2007).

4. Zatloukal $\mathrm{P}$, Petruzelka L, Zemanova $\mathrm{M}$, et al., Concurrent versus sequential chemoradiotherapy with cisplatin and vinorelbine in locally advanced non-small cell lung cancer: a randomized study, Lung Cancer 46 (1), 87-98 (2004).

5. Fournel P, Robinet $G$, Thomas $P$, et al., Randomized phase III trial of sequential chemoradiotherapy compared with concurrent chemoradiotherapy in locally advanced non-smallcell lung cancer: Groupe Lyon-Saint-Etienne d'Oncologie Thoracique-Groupe Francais de Pneumo-Cancerologie NPC 95-01 Study, J Clin Oncol 23 (25), 5910-5917 (2005).

6. Furuse K, Fukuoka M, Kawahara $M$, et al., Phase III study of concurrent versus sequential thoracic radiotherapy in combination with mitomycin, vindesine, and cisplatin in unresectable stage III non-small-cell lung cancer, J Clin Oncol 17 (9), 2692-2699 (1999).

7. Bradley J, A review of radiation dose escalation trials for non-small cell lung cancer within the Radiation Therapy Oncology Group, Semin Oncol 32 (2 Suppl 3), S111-113 (2005).

8. Fowler JF, Tome WA, Fenwick JD, et al., A challenge to traditional radiation oncology, Int J Radiat Oncol Biol Phys 60 (4), 1241-1256 (2004).

9. Cooper RA, Carrington BM, Loncaster JA, et al., Tumour oxygenation levels correlate with dynamic contrast-enhanced magnetic resonance imaging parameters in carcinoma of the cervix, Radiother Oncol 57 (1), 53-59 (2000).

10. Schutze $C$, Bergmann R, Yaromina A, et al., Effect of increase of radiation dose on local control relates to pre-treatment FDG uptake in FaDu tumours in nude mice, Radiother Oncol 83 (3), 311-315 (2007).

11. Piert $M$, Machulla HJ, Picchio $M$, et al., Hypoxia-specific tumor imaging with $18 \mathrm{~F}$ fluoroazomycin arabinoside, J Nucl Med 46 (1), 106-113 (2005).

12. Foo SS, Abbott DF, Lawrentschuk N, et al., Functional imaging of intratumoral hypoxia, Mol Imaging Biol 6 (5), 291-305 (2004).

13. Troost EG, Laverman P, Philippens ME, et al., Correlation of [18F]FMISO autoradiography and pimonidazole [corrected] immunohistochemistry in human head and neck carcinoma xenografts, Eur J Nucl Med Mol Imaging 35 (10), 1803-1811 (2008). 
14. Busk M, Horsman MR, Jakobsen S, et al., Imaging hypoxia in xenografted and murine tumors with 18F-fluoroazomycin arabinoside: a comparative study involving microPET, autoradiography, PO2-polarography, and fluorescence microscopy, Int J Radiat Oncol Biol Phys 70 (4), 1202-1212 (2008).

15. Bentzen SM, Theragnostic imaging for radiation oncology: dose-painting by numbers, Lancet Oncol 6 (2), 112-117 (2005).

16. Borst GR, Belderbos JS, Boellaard R, et al., Standardised FDG uptake: a prognostic factor for inoperable non-small cell lung cancer, Eur J Cancer 41 (11), 1533-1541 (2005).

17. Downey RJ, Akhurst T, Gonen M, et al., Preoperative F-18 fluorodeoxyglucose-positron emission tomography maximal standardized uptake value predicts survival after lung cancer resection, J Clin Oncol 22 (16), 3255-3260 (2004).

18. Vansteenkiste JF, Stroobants SG, Dupont PJ, et al., Prognostic importance of the standardized uptake value on (18)F-fluoro-2-deoxy-glucose-positron emission tomography scan in non-small-cell lung cancer: An analysis of 125 cases. Leuven Lung Cancer Group, J Clin Oncol 17 (10), 3201-3206 (1999).

19. Aerts HJ, van Baardwijk AA, Petit SF, et al., Identification of residual metabolic-active areas within individual NSCLC tumours using a pre-radiotherapy (18)Fluorodeoxyglucose-PET-CT scan, Radiother Oncol 91 (3), 386-392 (2009).

20. Mac Manus MP, Hicks RJ, Matthews JP, et al., Metabolic (FDG-PET) response after radical radiotherapy/chemoradiotherapy for non-small cell lung cancer correlates with patterns of failure, Lung Cancer 49 (1), 95-108 (2005).

21. Aerts HJ, Bosmans G, van Baardwijk AA, et al., Stability of 18F-deoxyglucose uptake locations within tumor during radiotherapy for NSCLC: a prospective study, Int J Radiat Oncol Biol Phys 71 (5), 1402-1407 (2008). 


\section{CHAPTER}

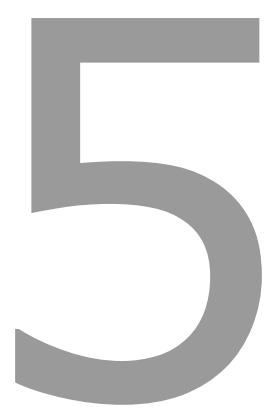

Stability of FDG uptake locations during treatment

Published in: Int J Radiat Oncol Biol Phys. 2008 Aug 1;71(5):1402-7

Stability of 18F-Deoxyglucose uptake locations within the tumor during radiotherapy for NSCLC: a prospective study

Hugo J.W.L. Aerts, Geert Bosmans, Angela A.W. van Baardwijk, Andre L.A.J. Dekker, Michel C. Oellers, Philippe Lambin, Dirk De Ruysscher 


\section{ABSTRACT}

\section{Background}

As individual tumors are heterogeneous, including for 18F-deoxyglucose (FDG) uptake and most likely for radioresistance, selective boosting of high FDG uptake zones within the tumor has been suggested. In order to do this, it is critical to know whether the location of these high FDG uptake patterns within the tumor remain stable during a course of radiotherapy.

\section{Methods}

Twenty-three patients with stage I-III non-small cell lung cancer (NSCLC) underwent repeated FDG-PET-CT scans before radical radiotherapy (day0), and at day7 and day14 of radiation. On all scans, high and low FDG uptake regions were auto-delineated using several standardized uptake value (SUV) thresholds, varying between $34 \%$ and $80 \%$ of the maximal SUV. Volumes and overlap fractions (OF) of these delineations were calculated to demonstrate the stability of the high FDG uptake regions during treatment.

\section{Results}

The mean OF of the $34 \%$ uptake zones at day0 with day7 and day 14 , was $82.8 \pm 8.1 \%$ and $84.3 \pm 7.6 \%$ respectively. The mean OF of the high uptake zones $(60 \%)$ was $72.3 \pm 15.0 \%$ and $71.3 \pm 19.7 \%$ at day 0 with day 7 and day 14 respectively. The volumes of the thresholds varied a lot, e.g. at day 0 the volume of the $60 \%$ zone was $16.8 \pm 20.3 \mathrm{~cm} 3$. Whereas the location of the high FDG uptake patterns within the tumor during treatment remained stable, the delineated volumes varied a lot.

\section{Conclusions}

The location of the low and high FDG uptake areas within the tumor remained stable during radiotherapy. This knowledge may enable selective boosting of high FDG uptake areas within the tumor. 


\section{INTRODUCTION}

Lung cancer remains one of the most frequent and lethal solid tumors ${ }^{1}$. Although the prognosis has improved, in locally advanced disease the 5-year survival is $20 \%$ at best ${ }^{2-5}$. The higher long-term survival observed with concurrent chemo-radiation compared to sequential chemotherapy and radiotherapy was due to improved local tumor control, emphasizing the importance of local tumor control on survival ${ }^{4,5}$. However, local tumor failure is still observed in a large amount of patients. Moreover, the major toxicity of concurrent regimens excludes its use in the majority of lung cancer patients because of their co-morbidity.

An important strategy to improve the effect of radiotherapy is to escalate the radiation dose, because a higher dose has been shown to yield a higher local control rate ${ }^{6}$. However, the total tumor dose is often limited due to constraints of surrounding normal tissue. Therefore innovative strategies are needed to obtain higher local tumor control rates. Until quite recently, tumors were viewed as homogeneous structures, with similar biological characteristics. It has become increasingly clear, however, that a tumor is heterogeneous for varying characteristics ${ }^{7-11}$. Indeed, molecular imaging studies showed significant differences in perfusion, hypoxia, cell density and proliferation within the tumor ${ }^{12}$. As these biologic characteristics are heterogeneously distributed throughout the tumor, the current practice to deliver a uniform dose-distribution seems illogical. It has been suggested to re-distributing dose, i.e. more resistant areas within the tumor could receive higher doses whilst reducing the dose to more susceptible zones, keeping normal tissue exposure similar ${ }^{12,13}$.

Molecular imaging might be useful to identify radioresistant areas. However, the resolution of molecular imaging is in the order of millimeters. This is a large scale compared to the heterogeneity of tumor tissue. For instance, the distance between well oxygenated tissue and hypoxic regions can be well below $100 \mu \mathrm{m}$, which is well below the resolution of Positron Emission Tomography (PET) ${ }^{14}$. The voxel intensity thus represents an average over the voxel and small highly hypoxic regions will not be observed, if they are in close proximity to well oxygenized tissue. At present, it is thus not clear whether the resolution of molecular imaging is sufficient to outline the biological heterogeneity in a meaningful way for dose painting.

Previously, we demonstrated that the maximal ${ }^{18}$ F-deoxyglucose (FDG) uptake does not remain stable during a course of radiotherapy, and a large intra-patient heterogeneity was found ${ }^{15}$. One of the essential remaining questions to be answered before selectively boosting parts of the tumor, is whether the locations within the tumor that show a high tracer uptake remain stable throughout a course of fractionated radiotherapy. As the uptake of FDG in the primary tumor is prognostic for survival of pa- 
tients with non-small cell lung cancer (NSCLC), both treated with surgery or radiotherapy ${ }^{16-20}$, we investigated the stability of the areas of FDG uptake in the primary tumor of NSCLC patients during their treatment with fractionated radiotherapy.

\section{MATERIAL AND METHODS}

\section{Patient characteristics}

Twenty-three patients with medically inoperable (stage I, $\mathrm{n}=2$ ) or locally advanced (stage III, $\mathrm{n}=21$ ) histologically confirmed proven NSCLC, with a WHO performance of status $0-1$ and good lung function (FEV1 $\geq 50 \%$ and DLCO $\geq 50 \%$ ), were included in this study, starting from December 2004 until April 2006. Nineteen patients received induction chemotherapy, consisting of three courses of Gemcitabine in combination with Cisplatin or Carboplatin. In the period this study was performed, induction chemotherapy followed by radiation treatment was the standard schedule. Therefore no concurrent chemotherapy was given. All patients enrolled in this study were treated as part of a feasibility study with High-dose Continuous Hyperfractionated Accelerated Radiotherapy (HI-CHART) ${ }^{21}$. The dose was delivered in three steps from $61.2 \mathrm{~Gy} / 34$ fractions/23 days to $64.8 \mathrm{~Gy} / 36$ fractions/24 days to $68.40 \mathrm{~Gy} / 38$ fractions/25 days (1.8 Gy BID with $8 \mathrm{~h}$ interval), using 3D conformal technique) ${ }^{21}$.The Medical Ethics Committee according to the Dutch law approved the trial. All patients gave written informed consent before entering this study.

\section{Radiotherapy simulation}

A treatment planning PET-CT scan in radiotherapy position was performed for simulation, and a 3D conformal treatment plan, using three to six coplanar $10 \mathrm{MV}$ photon fields, was calculated according to the ICRU guidelines ${ }^{22}$. Patients were positioned with both arms above the head and on an immobilization and patient laser marker system. Radiotherapy technologists trained specifically for this purpose always carried out the simulation. The PET-CT scanner used was a Siemens Biograph (SOMATOM Sensation-16 with an ECAT ACCEL PET scanner). An intravenous injection of ${ }^{18}$ F-FluoroDeoxy-Glucose (FDG) (Tyco Health Care, Amsterdam, The Netherlands) of (weight * $4+$ 20) $\mathrm{MBq}$ was followed by $10 \mathrm{ml}$ physiologic saline. After a 45 minutes uptake period, during which the patient was encouraged to rest, PET and CT images were acquired. A spiral CT with intravenous contrast was performed covering the complete thoracic region. 


\section{Radiotherapy planning}

Radiotherapy planning was performed on an XiO (Computerized Medical Systems, St Louis, Missouri) treatment planning system, using inhomogeneity corrections, based on a convolution algorithm. For all patients, the Gross Tumor Volume (GTV) and the Planning Target Volume (PTV) were defined, based on PET-CT data. Multiple GTV volumes were created in case of nodal involvement. The Clinical Target Volume (CTV) was defined as the GTV with a $5 \mathrm{~mm}$ margin incorporating microscopic disease. The heart and esophagus were excluded from the CTV. This CTV was subsequently expanded with $1 \mathrm{~cm}$ to the PTV to incorporate the internal respiratory motion and setup errors. Contouring of the lungs was carried out automatically by the treatment planning system. For the calculation of the mean lung dose (MLD), the volume of both lungs excluding the gross tumor volume (GTV) was used ${ }^{23}$. The spinal cord was drawn throughout the whole CT scan and was considered to be at the inner margin of the bony spinal canal. Dosimetric values were calculated on the basis of dose-volume histograms and dose distributions on each axial CT slice.

\section{Radiotherapy dose and normal tissue dose constraints}

For each patient, the radiation dose was escalated to a mean lung dose (MLD) of $19 \pm 1$ Gy while respecting a maximum spinal cord dose of $54 \mathrm{~Gy}$, with a maximal allowed total tumor dose (TTD) of $79.2 \mathrm{~Gy}$. There were no esophageal dose constraints. Radiotherapy was delivered twice a day (BID), and with a fraction size of $1.8 \mathrm{~Gy}$.

\section{Study design}

The PET-CT scan for treatment planning, before radiotherapy, was defined as day 0 . There were no scans used prior to induction chemotherapy. During the course of radiotherapy, the patients underwent repeat PET-CT scans, one week (day 7) and two weeks (day 14) after the start of treatment. At all time points, the scans were analyzed and delineated using the Siemens TrueD system (Siemens A.G., Darmstad, Germany). Using a rigid registration based on mutual information the images of day 7 and 14 were fused to day 0 . Using a region of interest (ROI) manager a manual region was selected around the tumor, avoiding high uptake areas like the heart. Within this region automatic contour delineations were generated using the thresholds $34,40,50,60,70$, $80 \%$ of the maximal SUV (SUV $\max$ ). The $34 \%$ threshold is indicative for GTV in most lung tumors, and is correlated with pathology and validated with phantom measurements ${ }^{22,24}$. The contour delineations were exported from TrueD as DICOM-RT structure sets. MATLAB (The MathWorks Inc.) was used to calculate the overlapping fractions (OF) and volumes of the structure sets. The overlap fraction was defined as the overlapping volume of the structure sets, divided by the volume of the smallest structure set: 


$$
O F=\frac{V_{1} \cap V_{2}}{\min \left(V_{1}, V_{2}\right)}
$$

where $V_{1}$ and $V_{2}$ stands for the volumes of the structure sets, $\bigcap$ denotes the union, and $\min \left(V_{1}, V_{2}\right)$ is the smallest of $V_{1}$ and $V_{2}$. The OF was computed for all tumors and thresholds in three dimensions. The OF varies between 0 (no overlap between the structures) and 1 (complete overlap of one structure with the other). A schematic representation of the OF calculation is shown in figure 1 . For all patients the PET-CT datasets were available at day 0 and day 7 . However, due to technical and logistical problems, only for $17 / 23(74 \%)$ patients a complete image dataset at all three time points was available.

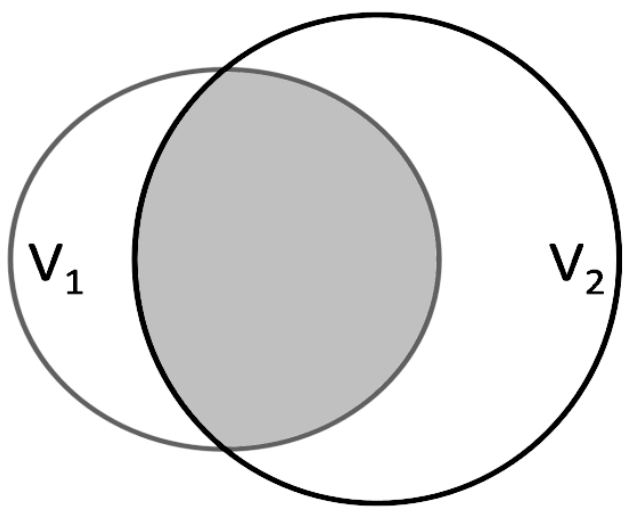

\section{Figure 1}

Schematic figure of the overlap fraction calculation, show the overlap of two volumes $\left(V_{1}\right.$ and $V_{2}$ ). The overlap fraction was defined as the overlap (gray area) of the two volumes, divided by the smallest volume (in this case $V_{1}$ ).

Data are expressed as mean \pm standard deviation (SD). Statistical differences between paired parameters from the three time points were evaluated in SPSS (SPSS 12.0.1 for Windows, Chicago, IL) using the Wilcoxon signed rank test. Differences were considered to be significant when the $p$-value was less than 0.05 . For the triple tests, a Bonferroni correction was applied and $p<0.0167$ was considered significant. It should be noted that the power of the test was limited due to the small number of patients $(n=23)$. However, the power was sufficient to find clinically relevant differences. 

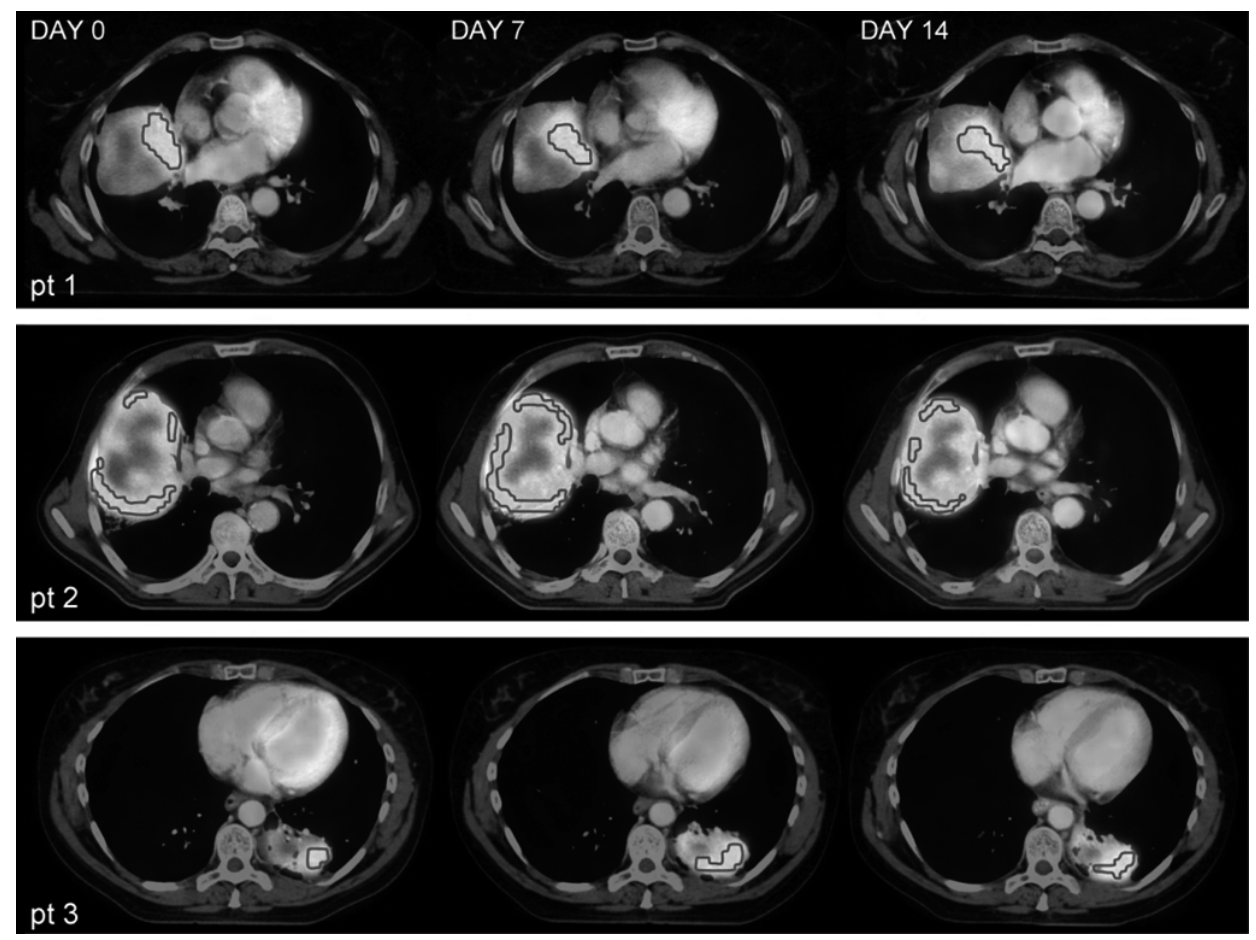

\section{Figure 2}

PET-CT images of three patients before treatment (day 0), and during treatment (day 7 and 14). The dark contours indicate the $60 \%$ of the maximal standardized uptake value $\left(S U V_{\max }\right)$ threshold. Visual inspection shows that the location of the hotspot remains at the same location during treatment, however the volume of the hotspot changes.

\section{RESULTS}

\section{Patient characteristics}

The mean age of the 23 patients (17 males, 6 females) was $66.1 \pm 9.0$ years [range: 52 80 years]. The mean prescribed TTD was $61.7 \pm 9.3 \mathrm{~Gy}$ [range: $46.8-79.2 \mathrm{~Gy}$ ] in $1.8 \mathrm{~Gy}$ fractions twice daily in a mean overall treatment time (OTT) of $24 \pm 4$ days [range: 17 31 days]. The corresponding MLD and maximal spinal cord dose were $16.1 \pm 3.6 \mathrm{~Gy}$ [range: $6.8-20.7 \mathrm{~Gy}$ ] and $46.2 \pm 13.1 \mathrm{~Gy}$ [range: $6.8-57.1 \mathrm{~Gy}$ ], respectively. The GTV of the primary tumor before radiotherapy was $91.9 \pm 146.9 \mathrm{~cm}^{3}$ [range: $0.4-660.2$ $\mathrm{cm}^{3}$ ]. The first repeat scan (day 7) was performed after the patient received on average a dose of $21.3 \pm 5.0 \mathrm{~Gy}$ (about 12 fractions) and the second scan (day 14) after $38.4 \pm$ 4.9 Gy (about 21 fractions). 


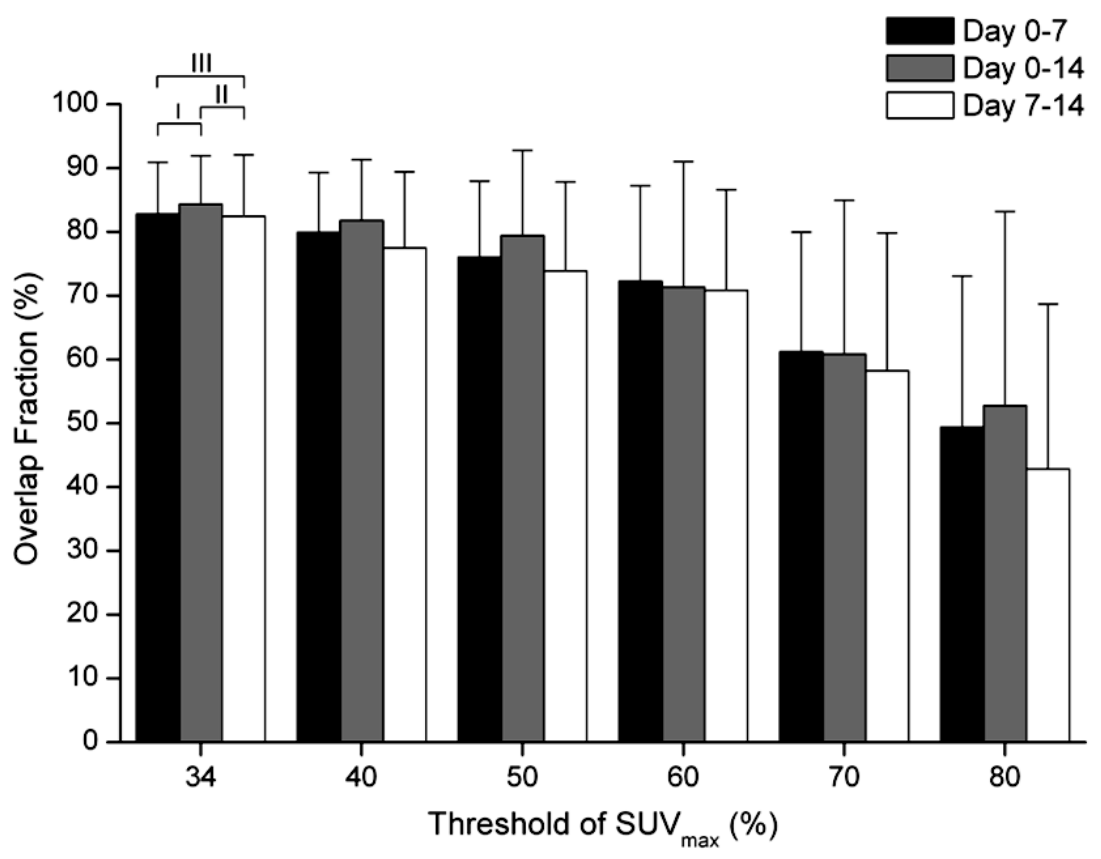

Figure 3

Overlap fractions (in \%) of day 0, 7 and 14 for the maximal standardized uptake value (SUV $V_{\max }$ ) thresholds ranging from 34-80\%. The error-bars indicate the standard deviation. The $34-60 \%$ hotspots show a large overlap (>70\%) for all time points. The I indicates the difference between the overlap of day 0-7 and day 0-14, II between day 0-14 and day 7-14, and III between day 0-7 and $7-14$ of the $34 \%$ threshold.

\section{Repeated PET-CT images}

In figure 2 representative PET-CT images of 3 patients before treatment (day 0), and during treatment (day 7 and 14) are shown. Visual inspection of the $60 \%$ SUV $_{\max }$ zones shows that the location of the hotspot remains in the same region of the tumor during treatment for all three patients. However, the volumes of the $60 \%$ threshold changed considerately during treatment, compared to day 0 .

\section{Overlap Fractions}

The overlap fractions of the different SUV thresholds are shown in figure 3. The 34$60 \%$ thresholds show a large overlap (>70\%) for all time points. The 70 and $80 \%$ thresholds show less overlap, however still the overlap is on average above $50 \%$. The limited OFs applying these thresholds were due to the low volumes of the $70 \%(8.6 \pm$ $9.4 \mathrm{~cm}^{3}$, range: $\left.0.5-36.8 \mathrm{~cm}^{3}\right)$ and $80 \%\left(3.5 \pm 3.9 \mathrm{~cm}^{3}\right.$, range: $\left.0.4-15.2 \mathrm{~cm}^{3}\right)$ thresholds on day 0 , often only a few image voxels. There was no significant difference between 


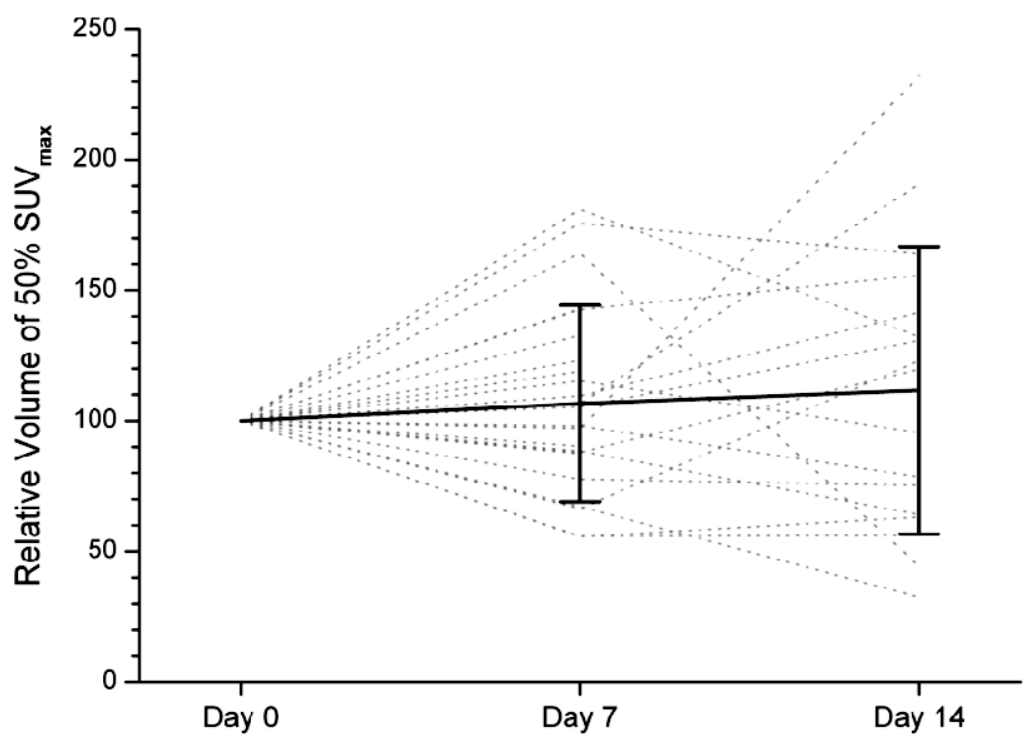

\section{Figure 4}

Volumes of the $50 \%$ maximal standardized uptake value $\left(S U V_{\max }\right)$ threshold, relative to the volume before treatment, of all patients were plotted vs. the number of days during radiotherapy (dotted lines). A large inter-patient variation is visible during radiotherapy. The bold black line is the average relative volume together with the standard deviation. The average varied nonsignificant between the time-points. Due to the normalization to the volumes of day 0 , no errorbars are present at this time-point.

the OFs of all the thresholds (34-90\%) of day 0-7 with the OFs of day 0-14 (Fig 3, example $34 \%$, see I). The same applied to the OFs of the thresholds of day 0-14 and day 7-14 (see II). In line with these results also no significant difference between the OFs of day 0-7 with day 7-14 was found (see III) (results not shown).

\section{Threshold Volumes changes during radiotherapy}

A remarkable heterogeneity of the changes in the volumes of the different thresholds was observed. To illustrate this heterogeneity the volumes of the $50 \%$ SUV $_{\max }$ threshold were plotted versus timepoints before and during radiotherapy (Fig. 4). The results of the $50 \%$ threshold are representative for the other thresholds. A striking interpatient variation is visible between the time-points, showing both a large SUV increases and decreases during time. In figure 5 all volumes of the different thresholds, relative to the volume before treatment (day 0), were plotted for the three timepoints. A large deviation of all volume changes during treatment can be seen.Again no significant difference can be seen between the relative volumes of day0, day 7 and day 14 (see I, II, III) (results not shown). 


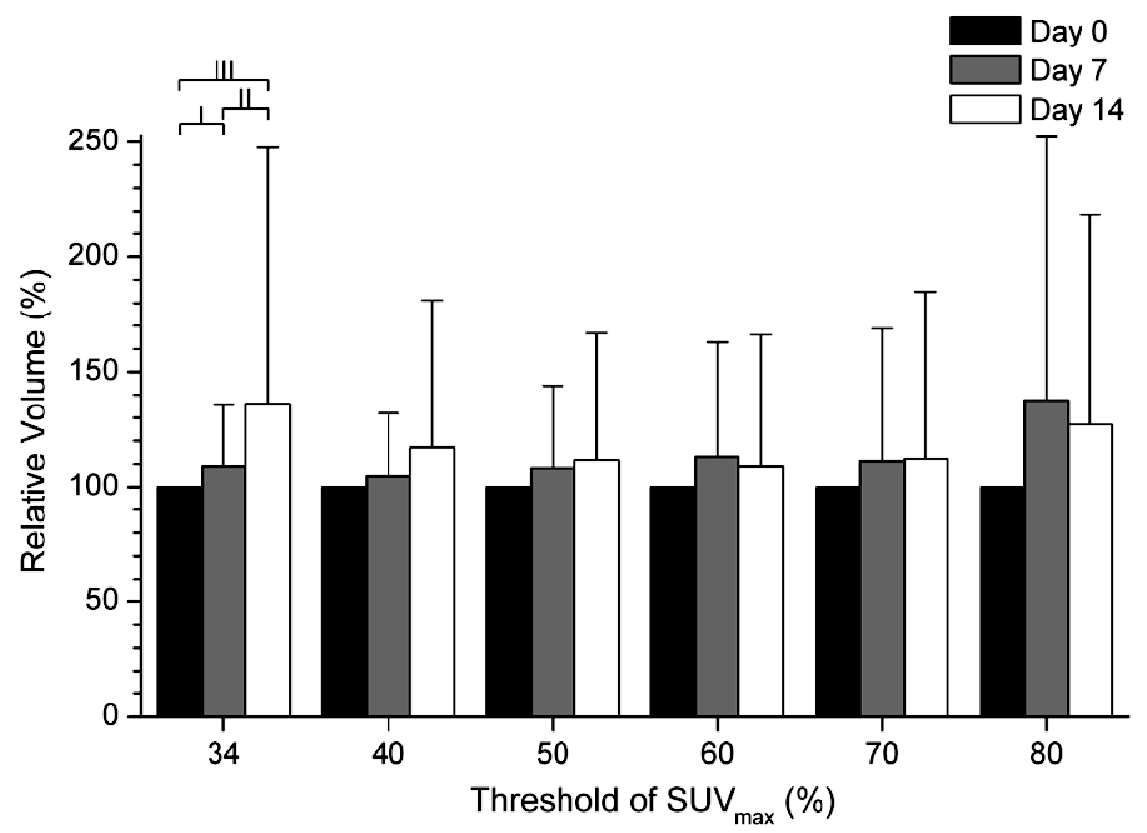

Figure 5

Volumes of thresholds of the maximal standardized uptake value $\left(S U V_{\max }\right)$, relative to the volume before treatment (day 0), were plotted vs. the number of days during treatment and for the SUV thresholds. The error-bars indicate the standard deviation. Due to the normalization to the volumes of day 0 , no error-bars are present at this time-point. A large deviation of all volumes during radiotherapy can be seen. The average varied non-significantly between all the time-points. I, II, and III indicate the differences between the relative volumes of the $34 \%$ SUV threshold. The I indicates the difference between the volume of day 0 and day 7 , II between day 7 and 14, and III between day 0 and 14 of the $34 \%$ threshold.

\section{DISCUSSION}

There is a growing interest in Radiation Oncology to selectively target radio-resistant areas within the tumor. This research is to a large extent driven by the availability of molecular imaging techniques that enable to visualize and quantify areas with different characteristics within the tumor ${ }^{7-10,12}$. The aim is to move away from the current paradigm of delivering a homogeneous physical dose distribution to the tumor to biological conformality by creating a heterogeneous dose pattern reflecting intra-tumor variation in biological factors of importance for response to radiation ${ }^{25-27}$. Before launching clinical trials, one main issue to be resolved is the question whether the areas within the tumor associated with a high FDG uptake remain stable during a course of radiotherapy. 
We decided to investigate the time-trends of the intra-tumor uptake of ${ }^{18} \mathrm{~F}$ deoxyglucose (FDG) in the primary tumor of patients with NSCLC treated with radical radiotherapy, as the maximal uptake of this tracer is prognostic for survival of patients with NSCLC, both treated with surgery or radiotherapy ${ }^{16-20}$. Moreover, results of our group suggest that the evolution of the maximum FDG uptake during radical radiotherapy for NSCLC may be prognostic for survival ${ }^{15}$.

Our results show that the mean overlap fractions (OF), which were used to demonstrate the stability in localization of FDG uptake regions during radiotherapy, of the low uptake zones before radiotherapy and at day 7 and 14 during therapy remained stable at about $83 \%$. Also the mean OF of the high uptake areas within the tumor remained stable throughout a course of radiation at about $71 \%$. However, the volumes varied considerably, e.g. the $60 \%$ threshold had a volume of $16.8 \pm 20.3 \mathrm{~cm} 3$. Bosmans et al also reported a large heterogeneity of tumor volume changes, where the delineation was based on CT images 28. We therefore concluded that the location of the high FDG uptake regions within the tumor during treatment remained stable, although the volumes of the thresholds can vary a lot between different time points.

To the best of our knowledge, these are the first data showing the stability of FDG uptake during radiotherapy for NSCLC. Indeed, although several papers have been published on theoretical radiation delivery to inhomogeneous parts of the tumor, none have investigated in real patients whether the areas remain stable during fractionated radiotherapy ${ }^{13,29-33}$. Our results show that although the volumes of the tumor and hence the high and low FDG uptake areas within the tumor may vary considerably during a course of radiotherapy, the location of the hot and cold spots within the tumor remain stable. This is not obvious, as a tumor is a complex, dynamic tissue that responds to injury. Our results nevertheless support the hypothesis that on a more macroscopic, i.e. voxel level, the areas with a high FDG uptake reflect intrinsic differences of parts of the tumor that are not easily disturbed by radiotherapy. Therefore, dose painting may be feasible, as repeated PET imaging during radiotherapy is not necessary to identify changes in the high uptake areas. Repeated CT scans taken at different time points to measure changes in tumor volume may be sufficient for this purpose 28 .

One may argue the choice of FDG as a tracer for radio-resistance. Indeed, FDG uptake in the tumor does not reflect a single biological characteristic of the tumor, but is influenced by many pathways that are related to therapy-resistance ${ }^{19}$. However, as FDG uptake in the primary tumor has repeatedly shown to affect the prognosis of patients with NSCLC ${ }^{16-20}$, and together with its wide availability, we believe that FDG is still a rational choice in this respect. However, this study may not be representative for other PET tracers, like FMISO or FLT, and comparable studies have to be repeated. 
The choice of the thresholds to define high and low uptake areas was based on several standardized uptake value (SUV) threshold based auto-delineations varying between $34 \%$ and $80 \%$ of the maximal SUV. Evidence on which threshold to choose is sparse 22 and the threshold value will depend on target to background ratio, reconstruction algorithm and type of scanner ${ }^{34}$. Nevertheless, there are studies showing a good correlation between pathology and PET-based auto-contouring for NSCLC ${ }^{22,24}$.For future boosting studies, by redistributing the dose within the tumor, the selected high dose region may be identified with FDG PET scans. However, the actual region to boost is still not completely clear. The region can be defined as a SUV above an absolute value (e.g. SUV > 5.0), however the region can also be defined as a threshold of SUV $\mathrm{mean}_{\text {or }}$

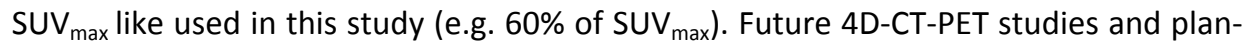
ning studies have to answer if CTV margins have to be applied to these regions, to correct for tumor motion and setup errors.

In conclusion, our results show that the high FDG uptake areas within the tumor remain stable during a course of radiotherapy, although the volumes may change considerably. This knowledge may further enable the design of clinical studies investigating the possibility of selective boosting of therapy-resistant areas within the tumor.

\section{ACKNOWLEDGEMENTS}

The TrueD system was kindly provided by Siemens. The authors would like to thank Wouter van Elmpt and Lucas Persoon for their contribution to the overlap fraction calculation. Also would we like to thank Ruud Houben for his statistical assistance.

\section{REFERENCES}

1. Jemal A, Siegel R, Ward E, et al. Cancer statistics, 2007. CA Cancer J Clin 2007;57:43-66.

2. Curran WJ, Scott CB, Langer CJ. Long-term benefit is observed in a phase III comparison of sequential vs. concurrent chemoradiation for patients with unresected stage III NSCLC. Proc Am Soc Clinic Oncol A; 2003.pp. 621 (Abstract A-2499).

3. Fournel $P$, Robinet $G$, Thomas $P$, et al. Randomized phase III trial of sequential chemoradiotherapy compared with concurrent chemoradiotherapy in locally advanced non-small-cell lung cancer: Groupe Lyon-Saint-Etienne d'Oncologie Thoracique-Groupe Francais de Pneumo-Cancerologie NPC 95-01 Study. J Clin Oncol 2005;23:5910-5917.

4. Furuse K, Fukuoka M, Kawahara M, et al. Phase III study of concurrent versus sequential thoracic radiotherapy in combination with mitomycin, vindesine, and cisplatin in unresectable stage III non-small-cell lung cancer. J Clin Oncol 1999;17:2692-2699. 
5. Zatloukal P, Petruzelka L, Zemanova M, et al. Concurrent versus sequential chemoradiotherapy with cisplatin and vinorelbine in locally advanced non-small cell lung cancer: a randomized study. Lung Cancer 2004;46:87-98.

6. Bradley J. A review of radiation dose escalation trials for non-small cell lung cancer within the Radiation Therapy Oncology Group.Semin Oncol 2005;32:S111-113.

7. Cooper RA, Carrington BM, Loncaster JA, et al. Tumour oxygenation levels correlate with dynamic contrast-enhanced magnetic resonance imaging parameters in carcinoma of the cervix. Radiother Oncol 2000;57:53-59.

8. Foo SS, Abbott DF, Lawrentschuk N, et al. Functional imaging of intratumoral hypoxia. Mol Imaging Biol 2004;6:291-305.

9. Garcia-Segura JM, Sanchez-Chapado M, Ibarburen C, et al. In vivo proton magnetic resonance spectroscopy of diseased prostate: spectroscopic features of malignant versus benign pathology. Magn Reson Imaging 1999;17:755-765.

10. Piert $M$, Machulla HJ, Picchio $M$, et al. Hypoxia-specific tumor imaging with 18Ffluoroazomycin arabinoside. J Nucl Med 2005;46:106-113.

11. Zhao S, Kuge $\mathrm{Y}$, Mochizuki $\mathrm{T}$, et al. Biologic correlates of intratumoral heterogeneity in $18 \mathrm{~F}$ FDG distribution with regional expression of glucose transporters and hexokinase-II in experimental tumor. J Nucl Med 2005;46:675-682.

12. Bentzen SM. Theragnostic imaging for radiation oncology: dose-painting by numbers. Lancet Oncol 2005;6:112-117.

13. Das SK, Miften MM, Zhou S, et al. Feasibility of optimizing the dose distribution in lung tumors using fluorine-18-fluorodeoxyglucose positron emission tomography and single photon emission computed tomography guided dose prescriptions. Med Phys 2004;31:1452-1461.

14. Dubois L, Landuyt W, Haustermans $\mathrm{K}$, et al. Evaluation of hypoxia in an experimental rat tumour model by [(18)F]fluoromisonidazole PET and immunohistochemistry. Br J Cancer 2004;91:1947-1954.

15. van Baardwijk A, Bosmans G, Dekker A, et al.Time trends in the maximal uptake of FDG on PET scan during thoracic radiotherapy.A prospective study in locally advanced non-small cell lung cancer (NSCLC) patients.Radiother Oncol 2007;82:145-152.

16. Borst GR, Belderbos JS, Boellaard R, et al. Standardised FDG uptake: a prognostic factor for inoperable non-small cell lung cancer. Eur J Cancer 2005;41:1533-1541.

17. Downey RJ, Akhurst T, Gonen M, et al. Preoperative F-18 fluorodeoxyglucose-positron emission tomography maximal standardized uptake value predicts survival after lung cancer resection. J Clin Oncol 2004;22:3255-3260.

18. Eschmann SM, Friedel G, Paulsen F, et al. Is standardised (18)F-FDG uptake value an outcome predictor in patients with stage III non-small cell lung cancer? Eur J Nucl Med Mol Imaging 2006;33:263-269.

19. van Baardwijk A, Dooms C, van Suylen RJ, et al. The maximum uptake of (18)Fdeoxyglucose on positron emission tomography scan correlates with survival, hypoxia inducible factor-1alpha and GLUT-1 in non-small cell lung cancer. Eur J Cancer 2007;43:13921398. 
20. Vansteenkiste JF, Stroobants SG, Dupont PJ, et al. Prognostic importance of the standardized uptake value on (18)F-fluoro-2-deoxy-glucose-positron emission tomography scan in non-small-cell lung cancer: An analysis of 125 cases. Leuven Lung Cancer Group. J Clin Oncol 1999;17:3201-3206.

21. De Ruysscher D, Wanders R, van Haren E, et al. HI-CHART: A phase I/II study on the feasibility of High-dose Continuous Hyperfractionated Accelerated Radiotherapy in patients with inoperable non-small cell lung cancer. Accepted for publication in Int J Rad Oncol Biol.

22. Daisne JF, Duprez $T$, Weynand $B$, et al. Tumor volume in pharyngolaryngeal squamous cell carcinoma: comparison at CT, MR imaging, and FDG PET and validation with surgical specimen. Radiology 2004;233:93-100.

23. Senan S, De Ruysscher D, Giraud P, et al.Literature-based recommendations for treatment planning and execution in high-dose radiotherapy for lung cancer.Radiother Oncol 2004;71:139-146.

24. van Baardwijk A, Bosmans G, Boersma L, et al. PET-CT-based auto-contouring in non-smallcell lung cancer correlates with pathology and reduces interobserver variability in the delineation of the primary tumor and involved nodal volumes. Int J Radiat Oncol Biol Phys 2007;68:771-778.

25. Apisarnthanarax $\mathrm{S}$, Chao KS. Current imaging paradigms in radiation oncology.Radiat Res 2005;163:1-25.

26. Bentzen SM. Radiation therapy: intensity modulated, image guided, biologically optimized and evidence based. Radiother Oncol 2005;77:227-230.

27. Ling CC, Humm J, Larson S, et al. Towards multidimensional radiotherapy (MD-CRT): biological imaging and biological conformality. Int J Radiat Oncol Biol Phys 2000;47:551-560.

28. Bosmans $G$, van Baardwijk A, Dekker $A$, et al. Intra-patient variability of tumor volume and tumor motion during conventionally fractionated radiotherapy for locally advanced nonsmall-cell lung cancer: a prospective clinical study. Int J Radiat Oncol Biol Phys 2006;66:748-753.

29. Alber M, Paulsen F, Eschmann SM, et al.On biologically conformal boost dose optimization.Phys Med Biol 2003;48:N31-35.

30. Chao KS, Bosch WR, Mutic S, et al. A novel approach to overcome hypoxic tumor resistance: Cu-ATSM-guided intensity-modulated radiation therapy. Int J Radiat Oncol Biol Phys 2001;49:1171-1182.

31. De Meerleer G, Villeirs G, Bral S, et al. The magnetic resonance detected intraprostatic lesion in prostate cancer: planning and delivery of intensity-modulated radiotherapy. Radiother Oncol 2005;75:325-333.

32. DiBiase SJ, Hosseinzadeh K, Gullapalli RP, et al. Magnetic resonance spectroscopic imagingguided brachytherapy for localized prostate cancer. Int J Radiat Oncol Biol Phys 2002;52:429-438.

33. Xing L, Cotrutz C, Hunjan S, et al. Inverse planning for functional image-guided intensitymodulated radiation therapy. Phys Med Biol 2002;47:3567-3578.

34. Daisne JF, Sibomana M, Bol A, et al. Tri-dimensional automatic segmentation of PET volumes based on measured source-to-background ratios: influence of reconstruction algorithms. Radiother Oncol 2003;69:247-250. 


\section{PART 2}

Non invasive imaging of Drug uptake:

From the bench to the clinic 



\section{CHAPTER}

\section{Imaging of drug uptake: In vitro imaging of Cetuximab}

Published in: Radiotherapy and Oncology 83 (2007) 326-332

Development and Evaluation of a Cetuximab-based Imaging Probe to target EGFR and EGFRvIII Hugo J.W.L. Aerts, Ludwig Dubois, Tilman M. Hackeng, Roel Straathof, Roland K. Chiu, Natasja G. Lieuwes, Barry Jutten, Sherry A. Weppler, Guido Lammering, Bradly G. Wouters, Philippe Lambin 


\section{ABSTRACT}

\section{Background and purpose}

The epidermal growth factor receptor (EGFR) is overexpressed in a significant percentage of human malignancies and its expression is associated with tumour aggressiveness and treatment resistance. The monoclonal antibody cetuximab (IMC-C225) blocks the ligand binding domain of EGFR with high affinity, preventing downstream signalling resulting in tumour growth inhibition. We developed and characterized a novel imaging probe using Oregon Green 488 labelled cetuximab to evaluate its usage as an imaging agent to target EGFR.

\section{Materials and Methods}

Cells with varying expression levels of EGFR or a mutant form of EGFR, called EGFRvIII, were used for in vitro validation. Thein vivo binding of labelled cetuximab to EGFR was also assessed ex vivo on tumour material.

\section{Results}

The development of Oregon Green 488 labelled cetuximab was successful, demonstrating binding to both EGFR and EGFRvIll in vitro. Accumulation was also found in vivo, which was confirmed by histopathology using anti-EGFR antibodies. However, significant mismatch highlights differences between drug delivery in vivo, and cell expression levels of EGFR.

\section{Conclusions}

The monoclonal antibody cetuximab represents a promising probe to evaluate the biologic and pharmacokinetic effects of in vivo cetuximab binding to EGFR. It not only visualizes the presence of the wild type EGFR, but also the presence of the mutant EGFRvIII. 


\section{INTRODUCTION}

The epidermal growth factor receptor (EGFR) plays an important role in tumour cell proliferation, differentiation and survival ${ }^{1,2}$. EGFR is highly expressed in a significant percentage of human malignancies and its expression is associated with tumour aggressiveness and treatment resistance ${ }^{3-6}$. Increasing evidence suggest that alterations within theEGFR gene may be as important as EGFR-overexpression to induce oncogenic effects. The most common variation is an in-frame deletion of exons 2-7 of the coding sequence resulting in a truncated mutant (EGFRvIII) which lacks a portion of the extracellular ligand-binding domain. Even though no ligand binding occurs, the tyrosine kinase in the intracellular portion is constitutively activated, leading to a receptor tyrosine autophosphorylation. EGFRvIII is expressed in certain tumours and its expression promotes tumour growth and therapy resistance ${ }^{7-9}$.

Blockade of EGFR function provides a promising approach for cancer treatment ${ }^{10-12}$. It has been shown that the human chimeric monoclonal antibody cetuximab (IMC-C225) blocks the ligand-binding domain of EGFR by binding to EGFR with high affinity, preventing downstream signalling which results in tumour growth inhibition ${ }^{13-16}$. Consequently, cetuximab has evolved as a promising new targeting agent in oncology and is increasingly used in combination with chemo-/radiotherapy as standard treatment of solid tumours and/or metastases ${ }^{14,17-19}$. Due to the heterogeneity of EGFR expression and its modulation during treatment, an imaging tool to monitor and visualize the binding of cetuximab non-invasively in cancer patients would be of great advantage, resulting in better patient selection for anti-EGFR targeted therapy. In addition, it could dramatically increase our knowledge of the clinical use of cetuximab and would help to optimize and individualize treatment.

A few studies have already reported on attempts to monitor cetuximab binding by using radioisotopes, where EGFR-positive tumours have been visualized both in animal models and in patients using ${ }^{99 \mathrm{~m}} \mathrm{Tc}{ }^{20,21}$ or ${ }^{64} \mathrm{Cu}{ }^{22}$ labelled cetuximab. However, limitations exist in using radioisotopes in the laboratory in a daily practice. Therefore, we developed an Oregon Green 488 labelled cetuximab imaging probe (cetuximab-OG) to assess the biological properties of cetuximab as an imaging and targeting agent. Here, we report the development and an in vitro and in vivo evaluation of a cetuximab based imaging probe to target EGFR and/or EGFRvIII. 


\section{MATERIAL AND METHODS}

\section{Oregon Green 488 labelling of cetuximab}

Cetuximab (2 mg/ml; Merck, Germany) was dialyzed against Hepes-buffered saline (HBS: $25 \mathrm{mM}$ Hepes $\mathrm{pH} 7.4,175 \mathrm{mM} \mathrm{NaCl}$ ) after which the protein concentration was determined by A280 measurement $\left(\varepsilon^{1 \%}{ }_{280 \mathrm{~nm}}=14.2\right)$. Cetuximab (12.1 $\mu \mathrm{M} ; 155 \mathrm{kDa} ; 25$ $\mathrm{ml}$ ) was incubated with succinimidyl-acetylthioacetate (SATA) at $25 \mu \mathrm{M}$ (final concentration). Cetuximab-(Lys)-ATA was de-acetylated by treatment with $50 \mathrm{mM}$ hydroxylamine, $2.5 \mathrm{mM}$ EDTA at $\mathrm{pH} 7.4$ for $1 \mathrm{~h}$ yielding cetuximab-(Lys)-SH. Incubation with 1 eq (mol/mol) of Oregon Green 488 (OG488)-maleimide resulted on average in a 1:1 labelling of cetuximab with OG488. Every reaction step was followed by analysis of 6 pmolessamples of the intermediate and final reaction product by MALDI-TOF mass spectrometry (Applied-Biosystems MALDI TOF/TOF 4800) in linear high mass mode using sinapinic acid as a matrix.

\section{In vitro cell model}

Human epidermoid carcinoma (A431), breast carcinoma (T47D), glioblastoma (U373, U87), cervix adenocarcinoma (HeLa), colorectal carcinoma (HT29, LS174T, HCT116) and prostate cancer (DU145) cells were obtained from the American Type Culture Collection (ATCC) and maintained under standard conditions in the appropriate medium supplemented with $10 \%$ Fetal Bovine Serum (Hyclone, Logan, UT). U373 cells were transfected with phßAc.EGFRvIII using Lipofectamine Plus (Invitrogen) according the manufacturers procedures. $48 \mathrm{~h}$ post transfection, geneticin selection was performed to obtain cells stably expressing EGFRvIII. The clone with the highest EGFRvIII expression, denoted U373-vIII(+), as assessed by immunoblotting, was selected for further experiments. A clone with no EGFRvIII expression was selected as control, denoted U373-vIII(-). For all experiments, cells were plated at a density of $0.5 \times 10^{6}$ per $6 \mathrm{~cm}$ dish the day before.

\section{Immunoblotting}

Cells were extracted in RIPA lysis buffer for $30 \mathrm{~min}$ on ice and protein concentrations were determined by a Bradford assay (Bio-Rad). Proteins were separated on a $10 \%$ SDS-polyacrylamide gel and transferred $\left(100 \mathrm{~V}, 1 \mathrm{~h}, 4^{\circ} \mathrm{C}\right)$ to nitrocellulose membranes (Amersham Corp.). Membranes were blocked (ON, $4^{\circ} \mathrm{C}$ ) with $5 \%$ Blotting Grade Blocker non-fat dry milk (BioRad) and subsequently incubated (2h, RT), with a 1:1000 dilution of the anti-EGFR rabbit polyclonal antibody (sc-03, Santa Cruz Biotechnology, Santa Cruz, CA). Proteins were visualized by a horseradish peroxidase method using Enhanced Chemiluminescence (Amersham Corp.). Mouse monoclonal $\beta$-Actin (Sigma) was used as loading control. 


\section{Immunofluorescence}

Immunocytochemistry was performed on cells grown on plastic $10 \mathrm{~cm}$ dishes until $80 \%$ confluency and incubated with cetuximab-OG $\left(60 \mathrm{nM}, 24 \mathrm{~h}, 37^{\circ} \mathrm{C}\right)$. Cells were washed 3 times using phosphate-buffered saline (PBS), fixed in $3 \mathrm{~mL}$ methanol on ice (10 min). Propidium iodide containing $1 \mu \mathrm{l} / \mathrm{ml}$ RNase (15min, RT) was used as nuclear marker. Immunohistochemistry was done on 7- $\mu \mathrm{m}$ frozen sections cut using a cryotome (Leica $\mathrm{CM} 3050 \mathrm{~S})$. After thawing, the sections were fixed in cold $\left(10 \mathrm{~min}, 4^{\circ} \mathrm{C}\right)$ acetone and rehydrated in PBS. In between the consecutive steps of the staining procedure, sections were rinsed with PBS. Sections were incubated overnight $\left(4^{\circ} \mathrm{C}\right)$ with the primary EGFR antibody (sc-03, Santa Cruz), followed by incubation ( $30 \mathrm{~min}, \mathrm{RT}$ ) with goat antirabbit Alexa fluor 594 conjugated IgG antibody (Molecular Probes). Cells and sections were mounted with Fluorescent Mounting Medium (DAKO) and analyzed using a Leica DM5000B fluorescence microscope (Leica Microsystems). The EGFR epitope binding sites of cetuximab (310-514) and sc-03 (1005-1016) are different ${ }^{23}$.

Quantitative analysis of the overlap between the cetuximab-OG and the EGFR antibody was assessed using the difference in fluorescence intensity. The images were normalized using a multiplication of the median intensity. The overlap area of cetuximab-OG uptake and EGFR expression was calculated using a difference less then $20 \%$ of the maximum.

\section{Flow cytometric analysis}

After three hours of incubation with cetuximab-OG at $37{ }^{\circ} \mathrm{C}$ (concentration ranging from 5.2fM - 5.2 $\mu \mathrm{M}$ ), cells were put on ice, washed with PBS and harvested. After resuspension in PBS, the cetuximab-OG labelled cells were fixed with fresh $2 \%$ paraformaldehyde ( $30 \mathrm{~min}, \mathrm{RT}$ ). Single cell suspensions were obtained by passing cells through $70 \mu \mathrm{m}$ nylon cell strainers (BD Biosciences). Mean fluorescence intensity was obtained using a FACSaria ${ }^{\circledR}$ flow cytometer (BD Biosciences) using FIT-C filter settings.

\section{Animal and tumour model}

Colorectal HT29 carcinoma cells were subcutaneously injected in NMRI-nu (nu/nu) female mice (28-32 gram) under anaesthesia. When tumours reached a volume of 500 $\mathrm{mm}^{3}$, animals were injected with $100 \mu \mathrm{g}$ cetuximab-OG via a lateral tail vein. After 5 days, to ensure good uptake of cetuximab-OG into the tumour tissue, the animals were sacrificed and the tumours were excised. All animal experiments were in agreement with national guidelines, approved by the Animal Ethics Committee of the University 'KU Leuven', Belgium. Isoflurane inhalation anaesthesia (induction $4 \%$ and maintenance $1-2 \%$ ) was used during all animal experiments. 


\section{RESULTS}

\section{Oregon Green 488 labelling of cetuximab}

Cetuximab was successfully labelled with OG488 at an average 1:1 stoichiometry (Fig. 1A). After incubation of cetuximab with succinimidyl-acetylthioacetate (SATA), MALDITOF mass spectrometry showed an approximate 100 Da increase of molecular weight (theoretical: $116 \mathrm{Da}$ ), showing that on average a 1:1 SATA-cetuximab reaction had occurred (data not shown). Deacetylation by hydroxylamine resulted in a mass decrease of approximately $50 \mathrm{Da}$ (theoretical: $42 \mathrm{Da}$ ) corresponding to the loss of one acetyl group and generation of a free sulfhydryl moiety on a lysine side-chain (data not shown). Incubation with 1 eq. OG488-maleimide for $2 \mathrm{~h}$ yielded cetuximab-OG in an average 1:1 stoichiometry according to MALDI-TOF mass spectral analysis (Fig. 1B). A total mass increase of 500 Da was observed, in good agreement with the mass increase of one OG488 (theoretical mass: $436 \mathrm{Da}$ ) coupled through one thioacetate linker (theoretical mass: $74 \mathrm{Da})$.

By using the described random labelling procedure it is possible that lysine side-chain modification might affect protein function. When analyzing the crystal structure of the Fab fragment of cetuximab, 23 lysine residues are visible in the light (blue) and heavy chain (grey) that can be modified ${ }^{23}$. However, only 1 lysine residue becomes apparent in the receptor-binding area of cetuximab (Fig. 1C) thereby minimizing chances on functional impairment of cetuximab by the labelling procedure. In addition, no significant differences $(P=0.408)$ between non-labelled and labelled cetuximab (79 $\pm 3.7 \%$ and $72 \pm 5 \%$ ) were seen in a clonogenic survival assay using a therapeutic dose of 60 nM on HT29 cells (Fig. 1D).

\section{In vitro analysis}

The cetuximab-OG binding to EGFR was first investigated in A431 cells using immunofluorescence (Fig. 2A). A clear membranous binding localization of cetuximab-OG was observed. In addition, we investigated if cetuximab-OG binds to EGFRvIII, using the U373-vIII(+) transfected cells. Also in these cells, membranous staining after incubation with cetuximab-OG was seen (Fig. 2B), indicating that cetuximab-OG binds not only EGFR, but also EGFRvIII. The EGFR fraction of the U373-vIII(+) transfected cells was limited, as assessed with immunofluorescence on the U373-vIII(-) cell line (data not shown). 
A
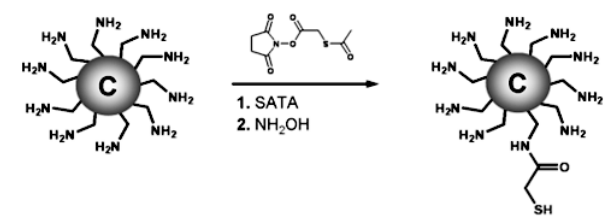

C

light chain

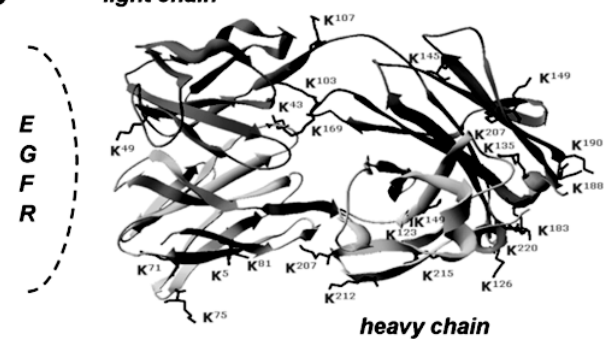

D

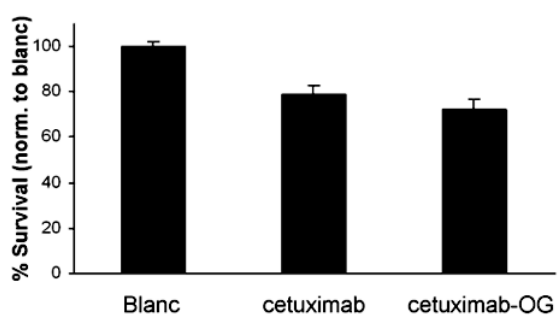

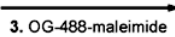

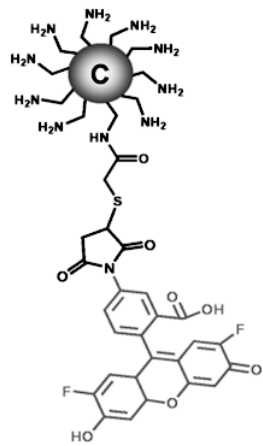

B
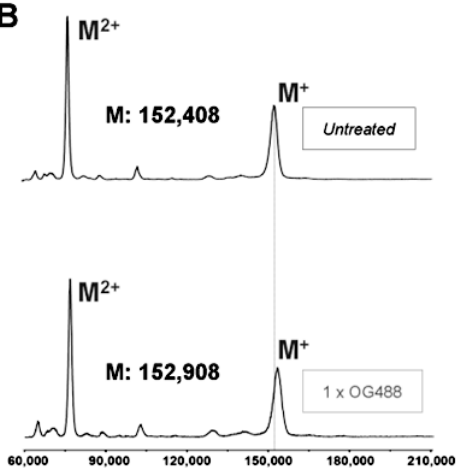

Figure 1

Labelling of cetuximab with Oregon Green-488. (A) Synthetic scheme of cetuximab (sphere C) labelling through its lysine side-chains $\left(-\mathrm{NH}_{2}\right)$ by coupling succinimidyl-acetylthioacetate (SATA) followed by deacetylation by hydroxylamine $\left(\mathrm{NH}_{2} \mathrm{OH}\right)$, and subsequent reaction with Oregon Green 488 (OG488)-maleimide. (B) Linear MALDI-TOF mass spectrum of unlabelled and OG488labelled cetuximab showing the single and double charged state of the protein. (C) Crystal structure of the Fab fragment of cetuximab, adapted from ${ }^{23}$, showing the 23 lysine residues in the light (dark) and heavy chain (light) that can be potentially modified. The binding site for EGFR is indicated. (D) Clonogenic survival of HT29 cells treated with non-labelled or OG-labelled cetuximab (60 nM), normalized to non treatment (blanc).

Quantitative binding of cetuximab to EGFR was determined using a flow cytometry saturation experiment. Expression of EGFR and EGFRvIII was assessed with a panel of cell lines using Western blotting (Fig. 3A). As expected A431 cells demonstrated high expression of EGFR, while T47D cells showed limited expression. The U373-vllI(+) cells showed high expression of the EGFRvIII, while the U373-vIII(-) cells only demonstrated EGFR expression in comparable levels as the U373 cells. The other investigated cell 


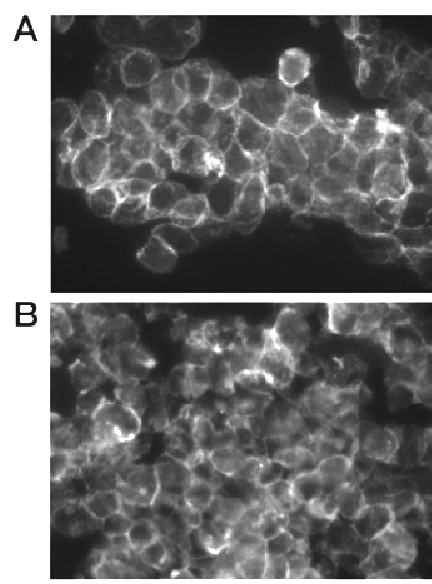

cetuximab-OG
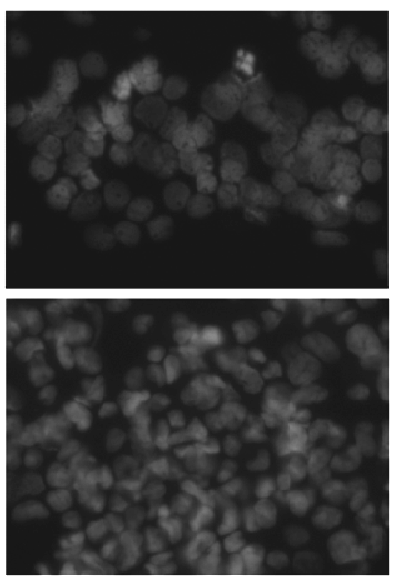

nuclear staining

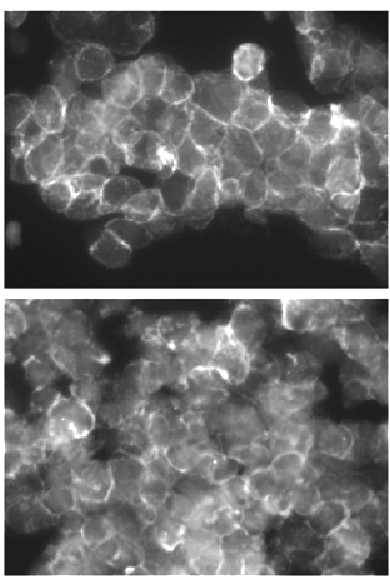

Merged

Figure 2

Immunofluorescence analysis of (A) A431 and (B) U373-vIII(+) cells incubated for $24 \mathrm{~h}$ with cetuximab-OG. Propidium iodide was used for nuclear staining.

lines showed different levels of intermediate EGFR expression. In order to find the optimal experimental binding conditions a saturation experiment with the high (A431) and low (T47D) EGFR expressing cells was performed (Fig. 3B). Both cell lines were incubated for 3 hours with cetuximab-OG, using a range of concentrations $(5.2 \mathrm{fM}$ to $5.2 \mu \mathrm{M})$. Clear cetuximab-OG binding was observed in A431 cells, while the binding to T47D cells was comparable to background levels. The cetuximab-OG saturation point for A431 cells was $5.2 \mathrm{nM}$. All other cell lines were analysed at this concentration to assure sufficient cetuximab-OG presence (Fig. 3C). T47D signals were used as negative control in all experiments. As shown previously, the cetuximab-OG binding to A431 cells was high and on average 50-fold higher compared with the negative control. Also binding to U373-vIII(+) was high (on average 40-fold), showing primarily binding of cetuximab-OG to EGFRvIII. The other clinically relevant cells investigated showed intermediate binding of cetuximab-OG. Comparison between the binding profile of cetuximab-OG and the respective EGFR expression levels as determined by a densitometric analysis, demonstrated a significant relationship $\left(r^{2}=0.842, p=0.001\right.$; data not shown).

\section{Ex vivo analysis}

To validate the use of the imaging agent in vivo, cetuximab-OG was injected in intermediate EGFR-expressing colorectal HT29 tumour bearing mice. 120h later, the animals were sacrificed, tumours were excised and prepared for immunohistochemistry. 
A

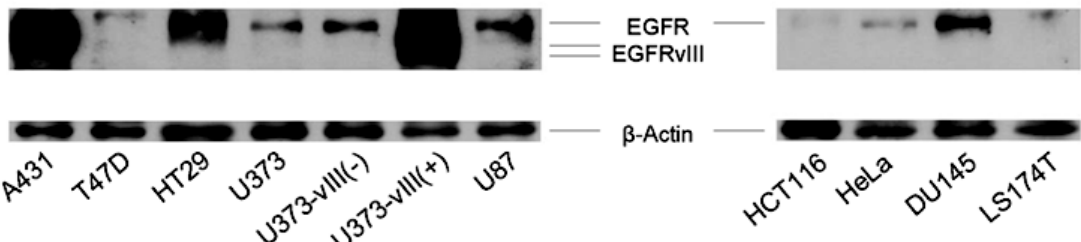

B

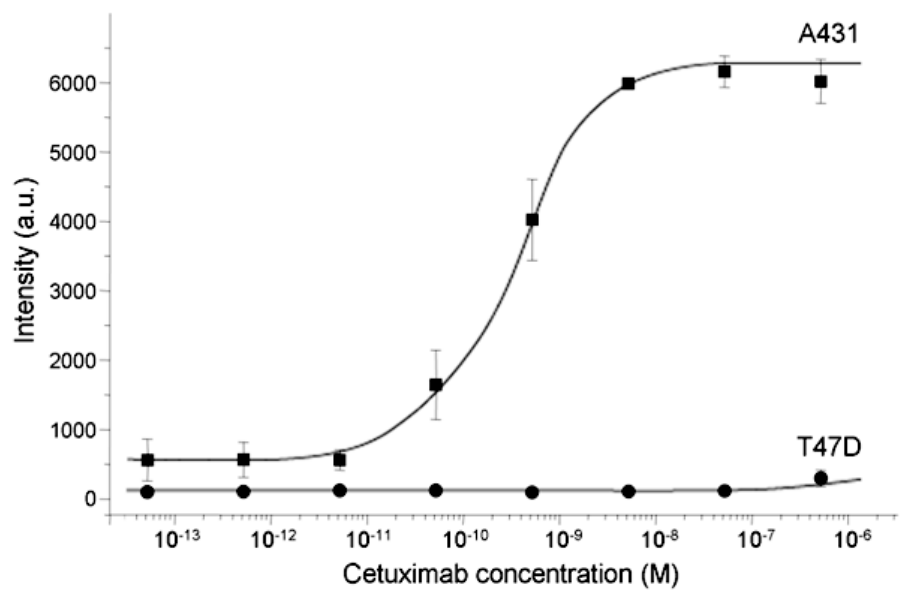

C

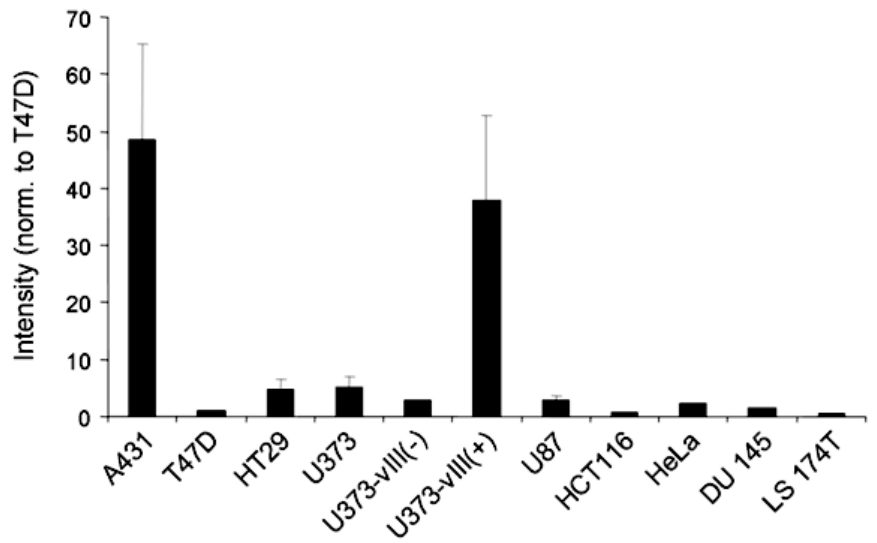

Figure 3

(A) Western blot analysis of EGFR and EGFRvIII expression in cells with varying EGFR expression levels. $40 \mu \mathrm{g}$ of protein was loaded on the gel. B-Actin was used as loading control. (B) FACS saturation experiment of cetuximab-OG binding to EGFR using A431 and T47D, showing a saturation concentration at $5.2 \mathrm{nM}$. Data points are shown as mean \pm SEM. (C) FACS analysis of cetuximabOG binding at saturation concentration to cell lines showing varying EGFR expression levels. Intensity levels of all cell lines were normalized to the intensity of the T47D cell line. With multiple measurements, the data points are shown as mean \pm SEM. 
Heterogeneous cetuximab-OG localization is shown in two representative tumour sections (Fig. 4).EGFR expression was assessed using the polyclonal EGFR antibody, which also showed a heterogeneous staining pattern. Merging both images demonstrated a partial mismatch between EGFR expression and cetuximab-OG tracer accumulation. The degree of match (and mismatch) was calculated by a difference map of representative sections of six tumours, where the intensity within $20 \%$ of the maximum was classified as overlap (a match), and larger as a mismatch. This resulted in an overlapping area of $83.4 \pm 5.9 \%$, a mismatch of cetuximab-OG positive / EGFR negative of $7.9 \pm$ $5.3 \%$, and a mismatch of cetuximab-OG negative / EGFR positive of $8.7 \pm 2.1 \%$.

\section{DISCUSSION}

We report the successful development of an Oregon Green 488 labelled cetuximab fluorescent imaging probe. Anticipating on future conjugation strategies of DTPAmaleimide synthons to couple cetuximab or more general to antibodies ${ }^{24}$ for use in MRI, PET, and SPECT imaging, we chose a coupling strategy, in which lysine side-chains were first converted to sulfhydryl moieties followed by reaction with sulfhydrylreactive maleimide-labels. No significant differences were found between labelled and non-labelled cetuximab preparations, indicating that cetuximab binding to EGFR is unaffected by the labelling procedure. To evaluate the properties of cetuximab as an imaging probe, we assessed the in vitro binding of cetuximab-OG to EGFR. Cell lines expressing varying levels of EGFR, showed a corresponding accumulation of cetuximabOG. We also demonstrated binding of cetuximab-OG to U373 cells transfected for EGFRvIll. Mamot et al showed binding of cetuximab fragments to EGFRvIll expressing tumour cells in vitro ${ }^{25}$. However, there is no conclusive evidence that the whole cetuximab complex binds to EGFRvill.

Upon injection of cetuximab-OG in tumour bearing mice, we showed successful fluorescent imaging of EGFR in the tumour specimen. However, the distribution of the cetuximab-OG binding was found to be very heterogeneous throughout the tumour. Ex vivo immunohistochemistry also showed heterogeneous staining patterns for EGFR. Using difference mapping, quantitative analysis of the overlap between EGFR expression and cetuximab-OG uptake level was performed, showing a mismatch of both high cetuximab-OG uptake with low EGFR expression, as visa versa. Possible explanations for this in vivo mismatch are multifactorial: poor drug delivery due to insufficient vasculature and perfusion of the tumour, dynamic tumour growth, and necrosis may limit the uptake of cetuximab. Also differences in internalization rates of the cetuximabEGFR complex may be cell type specific. However, the majority of cetuximab binding 
A
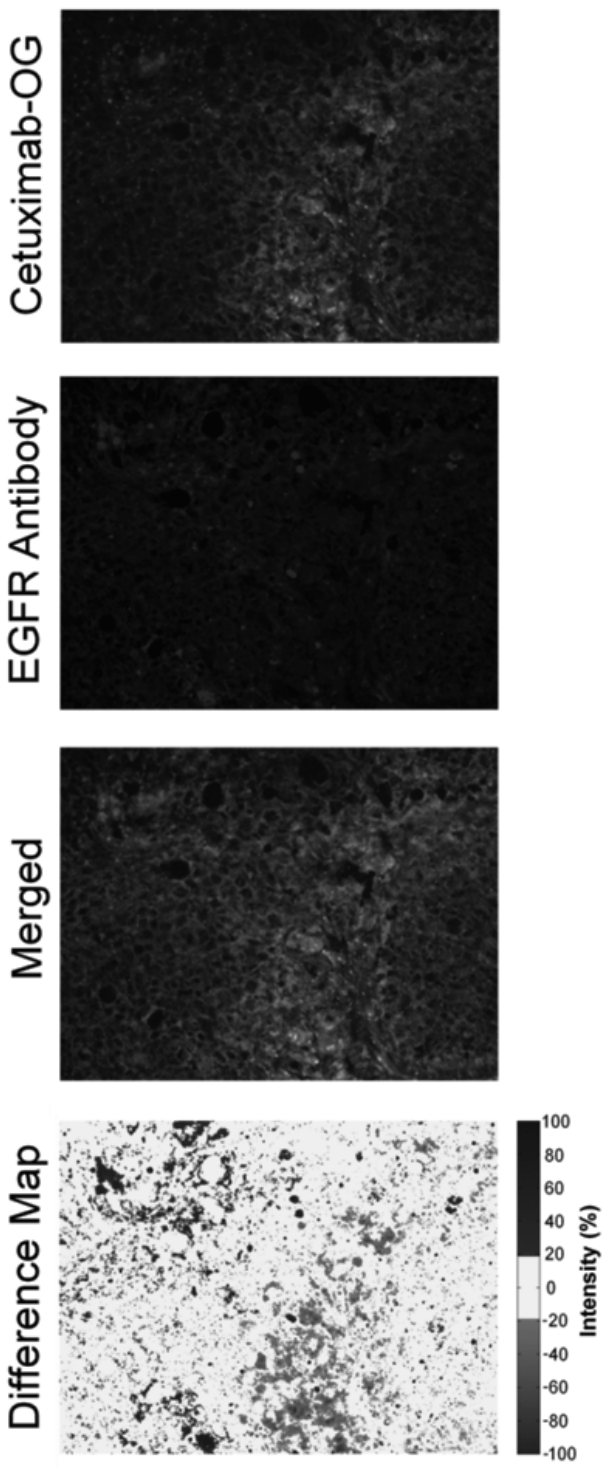

B
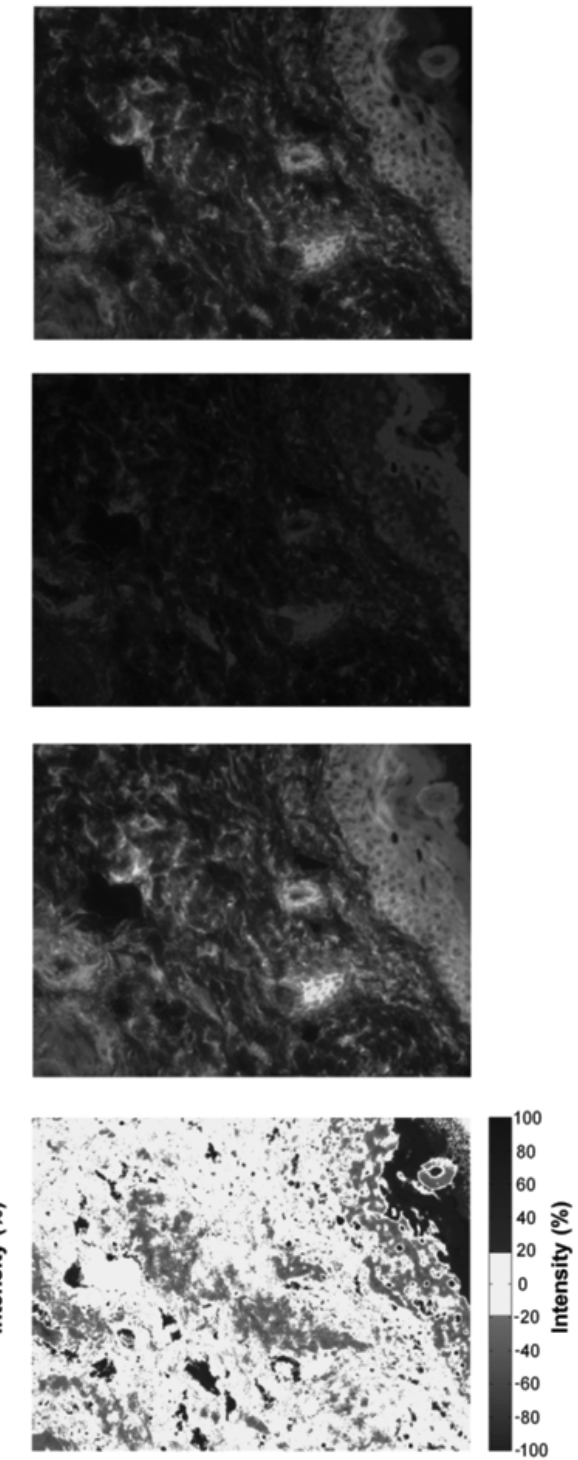

\section{Figure 4}

In vivo cetuximab-OG binding and ex vivo EGFR expression in HT29 tumours is shown in two representative tumour sections ( $A$ and $B$ ). A merged image shows the mismatch between cetuximabOG and EGFR expression. Quantitative mismatch was assessed using an intensity difference map, showing overlap in yellow, mismatch of cetuximab-OG positive / EGFR negative in green, and mismatch of cetuximab-OG negative / EGFR positive in red. 
was found at the cell membrane even at this time point. Importantly, these data indicate that cetuximab uptake is dependent upon tumour factors in addition to EGFR expression levels. This may help to explain the poor correlation often reported between cetuximab efficacy and EGFR expression ${ }^{26}$.

An increasing number of studies report on the use of cetuximab for imaging purposes, most often using SPECT and PET radioisotopes in experimental tumour models ${ }^{21,22}$. These studies however are limited by the short half life of the used isotopes ( ${ }^{99 \mathrm{~m}} \mathrm{Tc}: 6$ hours and ${ }^{64} \mathrm{Cu}: 12$ hours). This short half life might be sufficient to perform imaging studies in mice, but for imaging studies in humans, longer durations are required to reach optimal tumour to blood ratios in humans as the biologic half life of cetuximab is longer in humans compared to mice ${ }^{27}$. Based on the findings of Verel et $a l^{28}$, the positron emitter Zirconium-89 $\left({ }^{89} \mathrm{Zr}\right)$ exhibits several advantages for the use with cetuximab. One advantage is the long physical half life of ${ }^{89} \mathrm{Zr}$ (78 hour), sufficient for the long biologic half life of cetuximab in the blood pool. Another advantage is the labelling efficiency of cetuximab with ${ }^{89} \mathrm{Zr}$, because the immunoreactive fraction of the labelled complex stays above $95 \%{ }^{29}$, compared with $63 \%$ for ${ }^{64} \mathrm{Cu}^{22}$. Although the use of radioisotopes shows advantages when performing in vivo imaging, there is an urgent need for non-radioactive labelled probes for in vitro studies, to better understand the biological consequences of EGFR directed therapies.

\section{CONCLUSION}

We successfully developed an Oregon Green 488 labelled cetuximab imaging probe, which is able to bind EGFR and EGFRvIII in vitro, where quantitative binding of the cetuximab is correlated with EGFR expression levels. Furthermore, we demonstrated in vivo EGFR labelling after injection of cetuximab-OG. Thus, cetuximab-OG represents a promising imaging probe to further evaluate the binding properties of cetuximab and it can be used to visualize the presence of both EGFR and EGFRvIII. However,significant mismatch differences between cell EGFR expression levels and cetuximab uptake can be expected in vivo.

\section{ACKNOWLEDGEMENTS}

This work was supported by the Euroxy $6^{\text {th }}$ framework grant LSCH-CT-2003-502932 (LD, $\mathrm{PL})$. 


\section{REFERENCES}

1. Herbst RS, Review of epidermal growth factor receptor biology, Int J Radiat Oncol Biol Phys 59 (2 Suppl), 21-26 (2004).

2. Mendelsohn J, Targeting the epidermal growth factor receptor for cancer therapy, J Clin Oncol 20 (18 Suppl), 1S-13S (2002).

3. Akimoto $T$, Hunter NR, Buchmiller $L$, et al., Inverse relationship between epidermal growth factor receptor expression and radiocurability of murine carcinomas, Clin Cancer Res 5 (10), 2884-2890 (1999).

4. Lammering G, Molecular predictor and promising target: will EGFR now become a star in radiotherapy?,Radiother Oncol 74 (2), 89-91 (2005).

5. Eicheler W, Krause M, Hessel F, et al., Kinetics of EGFR expression during fractionated irradiation varies between different human squamous cell carcinoma lines in nude mice, Radiother Oncol 76 (2), 151-156 (2005).

6. Eriksen JG, Steiniche T and Overgaard J, The influence of epidermal growth factor receptor and tumor differentiation on the response to accelerated radiotherapy of squamous cell carcinomas of the head and neck in the randomized DAHANCA 6 and 7 study, Radiother Oncol 74 (2), 93-100 (2005).

7. Pedersen MW, Meltorn M, Damstrup L, et al., The type III epidermal growth factor receptor mutation. Biological significance and potential target for anti-cancer therapy, Ann Oncol 12 (6), 745-760 (2001).

8. Moscatello DK, Holgado-Madruga M, Godwin AK, et al., (1995), Vol. 55, pp. 5536-5539.

9. Sok JC, Coppelli FM, Thomas SM, et al., Mutant epidermal growth factor receptor (EGFRvIII) contributes to head and neck cancer growth and resistance to EGFR targeting, Clin Cancer Res 12 (17), 5064-5073 (2006).

10. Baselga $\mathrm{J}$ and Arteaga $\mathrm{CL}$, Critical update and emerging trends in epidermal growth factor receptor targeting in cancer, J Clin Oncol 23 (11), 2445-2459 (2005).

11. Herbst RS and Shin DM, Monoclonal antibodies to target epidermal growth factor receptor-positive tumors: a new paradigm for cancer therapy, Cancer 94 (5), 1593-1611 (2002).

12. Imai $\mathrm{K}$ and Takaoka A, Comparing antibody and small-molecule therapies for cancer, Nat Rev Cancer 6 (9), 714-727 (2006).

13. Huang SM, Bock JM and Harari PM, Epidermal growth factor receptor blockade with C225 modulates proliferation, apoptosis, and radiosensitivity in squamous cell carcinomas of the head and neck, Cancer Res 59 (8), 1935-1940 (1999).

14. Xiong $\mathrm{HQ}$, Rosenberg A, LoBuglio $\mathrm{A}$, et al., Cetuximab, a monoclonal antibody targeting the epidermal growth factor receptor, in combination with gemcitabine for advanced pancreatic cancer: a multicenter phase II Trial, J Clin Oncol 22 (13), 2610-2616 (2004).

15. Dittmann K, Mayer C and Rodemann HP, Inhibition of radiation-induced EGFR nuclear import by C225 (Cetuximab) suppresses DNA-PK activity, Radiother Oncol 76 (2), 157-161 (2005).

16. Krause M, Ostermann G, Petersen C, et al., Decreased repopulation as well as increased reoxygenation contribute to the improvement in local control after targeting of the EGFR by C225 during fractionated irradiation, Radiother Oncol 76 (2), 162-167 (2005).

17. Bonner JA, Harari PM, Giralt J, et al., Radiotherapy plus Cetuximab for Squamous-Cell Carcinoma of the Head and Neck, N Engl J Med 354 (6), 567-578 (2006). 
18. Huang SM and Harari PM, Modulation of radiation response after epidermal growth factor receptor blockade in squamous cell carcinomas: inhibition of damage repair, cell cycle kinetics, and tumor angiogenesis, Clin Cancer Res 6 (6), 2166-2174 (2000).

19. Milas L, Fan Z, Andratschke NH, et al., Epidermal growth factor receptor and tumor response to radiation: in vivo preclinical studies, Int J Radiat Oncol Biol Phys 58 (3), 966-971 (2004).

20. Schechter NR, Wendt RE, 3rd, Yang DJ, et al., Radiation dosimetry of 99mTc-labeled C225 in patients with squamous cell carcinoma of the head and neck, J Nucl Med 45 (10), 16831687 (2004).

21. Schechter NR, Yang DJ, Azhdarinia A, et al., Assessment of epidermal growth factor receptor with 99mTc-ethylenedicysteine-C225 monoclonal antibody, Anticancer Drugs 14 (1), 49-56 (2003).

22. Cai W, Chen $\mathrm{K}, \mathrm{He} \mathrm{L}$, et al., Quantitative PET of EGFR expression in xenograft-bearing mice using (64)Cu-labeled cetuximab, a chimeric anti-EGFR monoclonal antibody, Eur J Nucl Med Mol Imaging (2007).

23. Li S, Schmitz KR, Jeffrey PD, et al., Structural basis for inhibition of the epidermal growth factor receptor by cetuximab, Cancer Cell 7 (4), 301-311 (2005).

24. Dirksen A, Meijer EW, Adriaens W, et al., Strategy for the synthesis of multivalent peptidebased nonsymmetric dendrimers by native chemical ligation, Chem Commun (Camb) (15), 1667-1669 (2006).

25. Mamot C, Drummond DC, Greiser U, et al., Epidermal growth factor receptor (EGFR)targeted immunoliposomes mediate specific and efficient drug delivery to EGFR- and EGFRvIll-overexpressing tumor cells, Cancer Res 63 (12), 3154-3161 (2003).

26. Hebbar M, Wacrenier A, Desauw C, et al., Lack of usefulness of epidermal growth factor receptor expression determination for cetuximab therapy in patients with colorectal cancer, Anticancer Drugs 17 (7), 855-857 (2006).

27. Frieze DA and McCune JS, Current status of cetuximab for the treatment of patients with solid tumors, Ann Pharmacother 40 (2), 241-250 (2006).

28. Verel I, Visser GW, Boellaard R, et al., 89Zr immuno-PET: comprehensive procedures for the production of 89Zr-labeled monoclonal antibodies, J Nucl Med 44 (8), 1271-1281 (2003).

29. Perk LR, Visser GW, Vosjan MJ, et al., (89)Zr as a PET surrogate radioisotope for scouting biodistribution of the therapeutic radiometals (90)Y and (177)Lu in tumor-bearing nude mice after coupling to the internalizing antibody cetuximab, J Nucl Med 46 (11), 1898-1906 (2005). 


\section{CHAPTER}

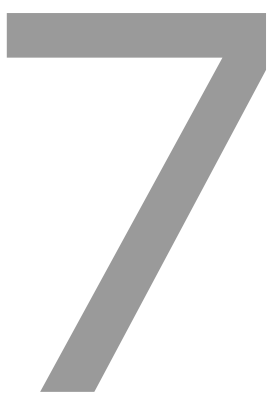

\section{Imaging of drug uptake: \\ Pre-clinical in vivo imaging of Cetuximab}

Published in: The Journal of Nuclear Medicine 2009; 50:123-131

Disparity between in vivo EGFR expression and Zirconium-89-labeled Cetuximab uptake assessed with PET

Hugo J.W.L. Aerts, Ludwig Dubois, Lars Perk, Peter Vermaelen, Guus A.M.S. van Dongen, Bradly G. Wouters, Philippe Lambin 


\section{ABSTRACT}

\section{Purpose}

The epidermal growth factor receptor (EGFR) is highly expressed in a significant number of human malignancies, and its expression is associated with tumor aggressiveness and overall treatment resistance. The monoclonal antibody cetuximab (IMC-C225) is increasingly used in clinical settings as a treatment modality in combination with more conventional therapies, such as radio- and chemotherapy. Currently, little is known about tumor-specific uptake and overall pharmacokinetics. Non-invasive quantification of cetuximab uptake could provide important diagnostic information for patient selection and therapy evaluation. To address this we have developed and validated a novel probe using cetuximab labeled with the long-lived positron emitter Zirconium-89 $\left({ }^{89} \mathrm{Zr}\right)$ for positron emission tomography (PET) imaging.

\section{Methods}

Tumor cell lines with varying EGFR expression levels were used for in vivo tumor imaging experiments. PET imaging with ${ }^{89} \mathrm{Zr}$-labeled cetuximab $(3.75 \pm 0.14 \mathrm{MBq})$ was performed on tumor-bearing NMRI-nu mice at multiple time-points post injection (ranging from 1 to 120 hours) and quantified by drawing regions of interest on selected tissues. Uptake was compared by biodistribution gamma-counting and ex vivo EGFR expression levels were quantified using Western blot analysis.

\section{Results}

Uptake of ${ }^{89} \mathrm{Zr}$-labeled cetuximab was demonstrated in the EGFR-positive tumors. However, the EGFR levels measured in vivo did not correlate with the relative signal obtained by PET. Tumor-to-blood ratios were significantly higher in the intermediate (compared with the high) EGFR-expressing cell lines starting from 24 hours post injection. Normal tissue uptake was unaffected by the different tumor types. Ex vivo gamma-counting experiments confirmed the observed in vivo PET imaging results. A similar disparity was found between ${ }^{89} \mathrm{Zr}$-labeled cetuximab tumor uptake and in vivo EGFR expression levels as demonstrated by Western blotting.

\section{Conclusions}

The ${ }^{89} \mathrm{Zr}$-labeled cetuximab imagingprobe is a promising tool for non-invasive evaluation of cetuximab uptake. Our results demonstrate a disparity between in vivo EGFR expression levels and cetuximab uptake. In a general sense, the results indicate a disparity between antibody uptake and expression levels of a biologic target in a tumor, suggesting that additional pharmacokinetic/pharmacodynamic mechanisms influence tumor delivery of this therapy. These additional mechanisms may explain why receptor expression levels alone are not sufficient to predict patient response. 


\section{INTRODUCTION}

Over the past few decades monoclonal antibodies (mAbs) have increasingly been used as targeting agents in oncology and are currently the subject of many important clinical trials ${ }^{1,2}$. One key biologic target for these mAbs is the epidermal growth factor receptor (EGFR), a member of the ErbB family. EGFR has been shown to play an essential role in the upregulation of tumor cell proliferation, differentiation and survival ${ }^{3,4}$. Activation of EGFR occurs when a ligand (e.g. epidermal growth factor or transforming growth factor alpha) binds to the extracellular ligand-binding domain, resulting in EGFR dimerization, internalization of the ligand-receptor complex and activation of the downstream signaling pathway. High EGFR expression is associated with tumor aggressiveness and overall treatment resistance, and is commonly found in a significant number of human malignancies ${ }^{5,6}$. As a result, EGFR targeting has been explored as a possible anti-tumor strategy, where the extracellular ligand-binding domain or the intracellular domain of the receptor is targeted with mAbs or small-molecule EGFR tyrosine kinase inhibitors (EGFR-TKIs) respectively ${ }^{7,8}$. Both these therapies disrupt the EGFR signaling pathway, resulting in inhibition of cell cycle progression, decreased angiogenesis, and a reduced metastatic invasion ${ }^{9}$.

Cetuximab (IMC-225, Erbitux) is an example of a mAbs which blocks the ligand-binding domain of EGFR with high affinity, thereby preventing downstream signaling ${ }^{9,10}$. Consequently, cetuximab has evolved as a promising new therapy agent in oncology and is increasingly used in clinical trials, mainly in combination with chemo- or radiotherapy 11,12 . The overall pharmacokinetics of cetuximab are slow (elimination rate ranging from 66 to 97 hours), partly due to the large size ( $\pm 152 \mathrm{kDa}$ ) of the antibody ${ }^{13}$. Despite the extensive use of cetuximab in clinical settings, much is still unknown about the patient-specific pharmacokinetics and pharmacodynamics of its tumor uptake. This results in a lack of knowledge about the relationship between dosage and therapeutic effect. Also, a disparity between EGFR expression levels and treatment outcome has been reported ${ }^{14-16}$, where even tumors negative for EGFR on biopsy responded to cetuximab treatment ${ }^{17}$. Furthermore, it has been shown in patients that mAbs which inhibit EGFR are associated in a dose-dependent manner with several dermatological side effects. In general, cutaneous side effects were most common, and patients experienced a mild to moderate eruption which did not require withdrawal of treatment ${ }^{18}$. On the other hand, an adequate cetuximab dose must be used to obtain sustained tumor uptake, since the liver has the capacity to absorb infused antibody up to $40 \mathrm{mg}$. Higher doses result in liver saturation and consequently in sufficient tumor uptake ${ }^{19}$. Therefore, the ability to monitor the in vivo cetuximab uptake in a non-invasive manner could significantly increase knowledge of cetuximab's clinical use as a targeting agent. We believe that this will eventually lead to the selective enrollment of patients and monitoring of cetuximab uptake before and during treatment. 
Using cetuximab itself as an imaging agent has several advantages for visualizing its distribution in normal and tumor tissues. Several groups have therefore investigated cetuximab-based non-invasiveimaging probes, the majority with SPECT and PET isotopes ${ }^{19-25}$. Some studies were limited by the use of relatively short half-life $\left(\mathrm{t}_{1 / 2}\right)$ isotopes $\left({ }^{99 \mathrm{~m}} \mathrm{Tc}\right.$ : $6 \mathrm{~h}$ and ${ }^{64} \mathrm{Cu}: 12 \mathrm{~h}$ ) which are incompatible with the long biologic half-life of cetuximab in the blood pool, and the relatively low immunoreactive fraction of the cetuximab radioimmunoconjugate ${ }^{22}$. Another PET isotope often suggested for antibody imaging is lodine-124, because its long half-life ( $t_{1 / 2}=100.3$ hours) allows imaging at later time points to acquire optimal information. However, an important disadvantage of lodine-124 is the rapid degradation of the radioiodinated mAb upon internalization, resulting in the rapid clearance of iodine from the target cells. This leads to PET images with less tumor contrast and does not reflect the actual tumor uptake of the antibody ${ }^{20}$. Based on the findings of Verel et al. ${ }^{26,27}$, the positron emitter Zirconium-89 $\left({ }^{89} \mathrm{Zr}\right)$ has ideal characteristics for usage with antibodies ${ }^{28} .{ }^{89} \mathrm{Zrdemonstrates}$ a long physical half-life ( $t_{1 / 2}=78$ hour) and, upon internalization of the antibody, is trapped intracellularly in lysosomes (phenomenon of residualization) ${ }^{29,30}$. Moreover, radiolabeling of cetuximab with ${ }^{89} \mathrm{Zr}$ does not influence its binding potential to EGFR, resulting in an immunoreactive fraction of about $95 \%{ }^{28}$. For these reasons, ${ }^{89} \mathrm{Zris}$ an ideal radionuclide for the labeling of slow kinetic intact antibodies with high quality standards, and is successfully used in pre-clinical ${ }^{31}$ and clinical settings ${ }^{20,32,33}$.

Here we report on the development and testing of the imaging probe ${ }^{89} \mathrm{Zr}$-labeled cetuximab, which was validated pre-clinically in mice bearing tumors with varying EGFR expression. Using PET imaging we assessed the in vivo cetuximab uptake levels and correlated those with the in vivo EGFR expression levels as determined by Western blotting.

\section{MATERIAL AND METHODS}

\section{Synthesis of $89 \mathrm{Zr}$-labeled Cetuximab}

${ }^{89} \mathrm{Zr}$ was produced by a $(\mathrm{p}, \mathrm{n})$ reaction on natural yttrium-89 $\left({ }^{89} \mathrm{Y}\right)$ as described by Verel et al. $^{27} .{ }^{89} \mathrm{Zr}$ labeling of the monoclonal antibody (mAb) cetuximab has been achieved as previously reported ${ }^{28}$. Briefly, desferrioxamine B (Df) was succinylated ( $N$-sucDf), temporarily filled with iron $\left(\mathrm{Fe}^{3+}\right)$, and coupled to cetuximab using a tetrafluorophenol$N$-sucDf ester. The premodified mAb was purified on a PD10 column after removal of $\mathrm{Fe}^{3+}$ by transchelation to ethylenediaminetetraacetic acid (EDTA). Approximately one $N$-sucDf moiety was coupled per cetuximab, assessed by using ${ }^{59} \mathrm{Fe}$. Next, $N$-sucDfcetuximab was labeled with ${ }^{89} \mathrm{Zr}$ in HEPES buffer at $\mathrm{pH} 7.0$ and again purified on a PD10 column to remove unbound ${ }^{89} \mathrm{Zr}$. The HPLC-isolated fractions demonstrated a radio- 
chemical purity in the order of $98 \%$. Specific activity was typically in the range of 78 $\mathrm{MBq} / \mathrm{mg}$.

\section{Animal and Tumor Model}

Exponentially growing human epidermoid (A-431; ATCC CRL-1555), breast (T-47D; ATCC HTB-133), glioblastoma (U-373 MG; ATCC HTB-17) and colorectal (HT-29; HTB-38) carcinoma cells were cultivated in the appropriate medium supplemented with $10 \%$ fetal bovine serum and incubated in a humidified $5 \% \mathrm{CO}_{2}$ chamber at $37^{\circ} \mathrm{C}$. Adult NMRI-nu (nu/nu) female mice (28-32 g) were inoculated subcutaneously in the lateral flanks with $3 \times 10^{6}$ cells resuspended in $100 \mu$ growth medium. Tumors were measured using a Vernier Caliper in three orthogonal tumor diameters ( $A, B$ and $C$ ), each corrected for the thickness of the skin, and tumor volumes were calculated based on the formula $A \times B \times C \times \pi / 6$. Once tumor volumes reached an average of $400 \mathrm{~mm}^{3}$, animals were injected with ${ }^{89} \mathrm{Zr}$-cetuximab (3.75 $\left.\pm 0.14 \mathrm{MBq}\right)$ via the lateral tail vein. All procedures were performed according to the guidelines defined in "Principles of laboratory animal care" (NIH publication no. 86-23, revised 1985). All animal experiments were in agreement with national guidelines, approved by the Animal Ethics Committee of the KU Leuven University, Belgium. Animals were given food and water ad libitum for the duration of the experiments. Isoflurane inhalation anesthesia ( $2 \%$ isoflurane in $100 \% \mathrm{O}_{2}$ at a flow rate of $1.5 \mathrm{~L} / \mathrm{min}$ ) was used during all animal experiments.

\section{PET Imaging and Analysis}

PET imaging was performed on a Focus 220 MicroPET (Concorde Microsystems Inc., Knoxville, TN) with an axial field of view of $7.6 \mathrm{~cm}$ and a resolution of approximately $1.4 \mathrm{~mm}$. For each animal, a custom-built alginate mold was made to obtain reproducible positioning in the opening of the camera between the imaging time points. Wholebody scanning was performed with 95 axial slices each of $0.796 \mathrm{~mm}$ in a single field of view, yielding radioactivity concentration measurements in voxels of 0.633 by 0.633 by $0.796 \mathrm{~mm}$. After injection of $3.75 \pm 0.14 \mathrm{MBq}{ }^{89} \mathrm{Zr}$-cetuximab (average dose of $100 \mu \mathrm{g}$ ) into the lateral tail vein for each animal a $25-\mathrm{min}$ emission scan was performed (5 frames each of $5 \mathrm{~min}$ ) at 1, 4, 24, 48, 72 and 96 hours post injection (p.i.) with correction for random counts, dead time and decay. For the HT-29 and U-373 MG an additional scan at $120 \mathrm{~h}$ p.i. was also available. Each emission scan was preceded by a $10-$ min transmission scan using external rods of ${ }^{57} \mathrm{Co}$ for subsequent attenuation correction. Three-dimensional list-mode data were acquired using an energy window of 350$750 \mathrm{keV}$ and a coincidence timing window of $6 \mathrm{~ns}$. The data were then sinogrammed and reconstructed iteratively in a $128 \times 128 \times 95$ matrix using attenuation weighted 2DOSEM (Fourier rebinning, 16 subsets, 10 iterations and 5 EM iterations). The images were visualized with ASIPro VM software (version 6.3.3.0; Concorde Microsystems Inc., Knoxville, TN) and analyzed using MATLAB (Version 7.3, MathWorks Inc., Natick, NA). 
For each data set, three-dimensional regions of interest (3D-ROIs) were manually drawn over the blood (heart outflow area), lungs, liver, kidneys, muscle, and tumors. Mean and maximum activity data (in $\mathrm{Bq} / \mathrm{ml}$ ) of the voxels within the ROls were obtained. All data were corrected for ${ }^{89} \mathrm{Zr}$ decay towards injection and the injected dose (ID), and are therefore expressed as \%ID/ml. Tumor-to-blood uptake ratios were calculated by dividing the activity data within the tumor ROI by the activity value within the blood ROI drawn on the outflow area of the heart.

\section{Immunoblotting}

Minced tumor pieces and cell pellets obtained from a $6 \mathrm{~cm}$ culture dish were extracted in RIPA lysis buffer (150 mM NaCl, 1\% NP-40, 0.5\% sodium deoxycholate, $0.1 \%$ SDS, 50 $\mathrm{mM}$ Tris- $\mathrm{HCl} \mathrm{pH} \mathrm{7.5,} 1 \mathrm{X}$ protease inhibitor cocktail) for 30 min on ice, and protein concentrations were determined by a Bradford assay (Bio-Rad). Proteins were separated on a $10 \%$ SDS-polyacrylamide gel and blotted onto a nitrocellulose membrane (Amersham Corp.) by electrotransfer. The membrane was blocked with $5 \%$ Blotting-Grade Blocker non-fat dry milk (Bio-Rad). Blots were probed with antibodies directed against EGFR (Santa Cruz Biotechnology) or $\beta$-Actin (Sigma). Bound antibodies were visualized using HRP-linked secondary antibodies (anti-rabbit [Cell Signaling Technologies] or anti-mouse [Sigma]) and ECL luminescence (Amersham Corp.).

\section{Biodistribution}

Following the last PET acquisitions, animals were sacrificed under anesthesia and dissected. Blood, tumors, skin, sternum, heart, thymus, salivary glands, thyroid, trachea, lung, esophagus, liver, gal bladder, stomach, pancreas, spleen, ileum, colon, bladder, kidney, muscle, thighbone, brown fat, eyes, ears, cerebrum, cerebellum, medulla oblongata, fat and tail were washed and flushed with saline, weighed, and the amount of radioactivity in each tissue was assessed in a $\mathrm{Y}$-well counter (1480 Wallac Wizard 3" Automatic Gamma Counter, PerkinElmer, USA). Tumors were cut into halves, one for quantification of ${ }^{89} \mathrm{Zr}$-cetuximab uptake and one rapidly frozen for protein isolation. Disintegrations for each sample were collected over one minute using an energy window between 48 and $1354 \mathrm{keV}$. To correct for decay, injection standards were counted simultaneously. Radioactivity uptake was calculated as the percentage of the injected dose per gram of tissue (\%ID/g).

\section{Statistics}

All statistical analyses were performed with SPSS 12.0.1 for Windows (SPSS Inc., 2003, Chicago, USA). Graphs were drawn using Origin 6.0 (Microcal Software Inc., 1999, Northampton, USA). Correlations among the groups were assessed using a nonparametric independent samples test (Kruskal-Wallis test). Further, to determine the 
statistical significance of differences between two independent groups of variables, a non-parametric Mann-Whitney U-test for small groups was used. For all tests, $p<0.05$ was considered significant.

\section{RESULTS}

\section{In Vivo Uptake of 89Zr-Cetuximab}

To evaluate the potential of ${ }^{89} \mathrm{Zr}$-cetuximab as an imaging agent, four cell lines with variable levels of EGFR expression were injected into NMRI-nu mice to form xenografts. A-431 was selected for high EGFR expression, U-373 MG and HT29 for intermediate, and T-47D for low EGFR expression, as previously described ${ }^{16}$. The in vivo binding of ${ }^{89} \mathrm{Zr}$-cetuximab was assessed by performing PET imaging at 72 hours post injection (72 h p.i.) on tumor-bearing mice after injection of $100 \mu \mathrm{g}{ }^{89} \mathrm{Zr}$-cetuximab (figure 1). A clear accumulation of ${ }^{89} \mathrm{Zr}$-cetuximab was found in the EGFR-positive tumors (U373 MG, HT-29, and A-431) compared with the low EGFR-expressing tumors (T-47D). Remarkably, a higher ${ }^{89} \mathrm{Zr}$-cetuximab uptake was demonstrated in the intermediate EGFR-expressing U-373 MG and HT-29 tumors compared with the high EGFRexpressing A-431 tumor. The uptake in the low EGFR-expressing tumor T-47D was comparable with that in the surrounding tissues. A clear accumulation was seen in normal tissues like the liver, due to the hepatobiliar clearance of the antibody. Notable uptake was also found in the kidney.

Imaging at several time points post injection was performed to assess the time-activity relationship of ${ }^{89} \mathrm{Zr}$-cetuximab uptake in the tumor. Figure 2 shows a representative time series of PET images of an HT-29 tumor-bearing mouse. From $24 \mathrm{~h}$ p.i., a clear localization of the tumors was seen above background uptake. The ${ }^{89} \mathrm{Zr}$-cetuximab uptake accumulated with time and the highest tumor uptake was reached around 72-96 h p.i. Furthermore, uptake of ${ }^{89} \mathrm{Zr}$-cetuximab was heterogeneously distributed within the tumor. Uptake in the outflow area of the heart decreased with time. Uptake in the liver also decreased, but more gradually so than that in the heart. 


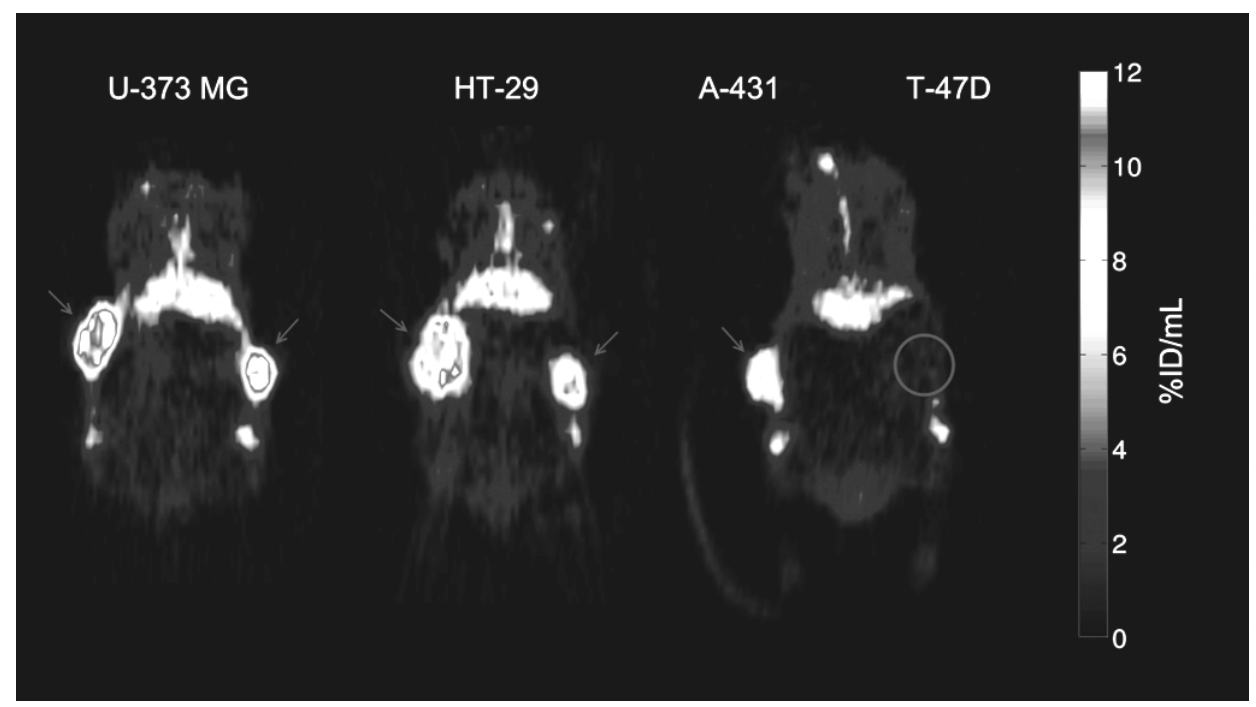

\section{Figure 1}

Representative microPET images 72 hours after injectionof ${ }^{89} \mathrm{Zr}$-cetuximab. Mouse bearing U-373 MG (left) or in HT-29 (center) tumors in both flanks. Mouse (right) bearing an A-431 and a T-47D (circled) tumor in the right and left flank respectively. The arrows indicate the tumors. Images at the mid-plane cross-section through the tumor are shown. The images are corrected for injected dose and decay, represented as \%ID/ml. High uptake is found in the EGFR-expressing tumors (HT29, U-373 MG, A-431), while the uptake of the low EGFR-expressing tumor (T-47D) is comparable with the overall uptake in surrounding normal tissue. ${ }^{89} \mathrm{Zr}$-cetuximab uptake was also found in the catabolic organs: in the liver and kidneys (below tumors).

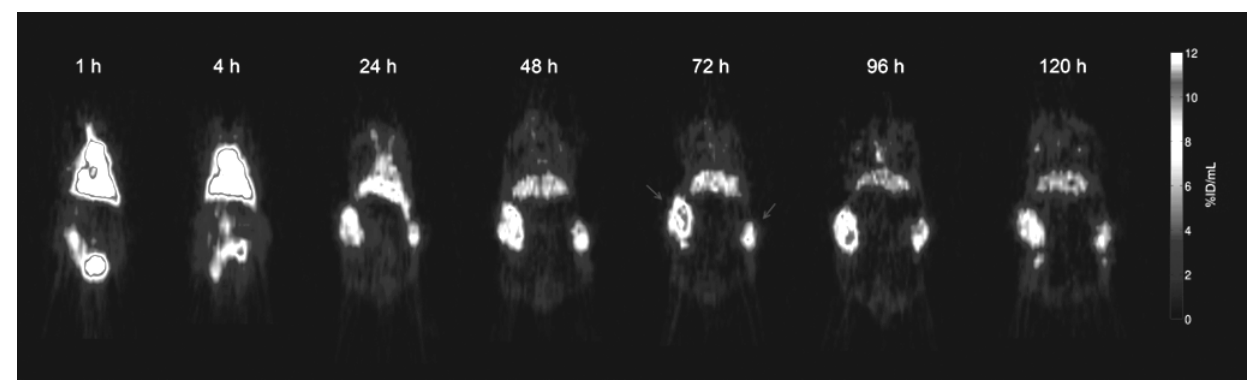

\section{Figure 2}

Time series of representative microPET images at indicated time points post injection of ${ }^{89} \mathrm{Zr}$ cetuximab of a HT-29 tumor-bearing mouse. The red arrows indicate the tumors. Uptake levels plateaued from 72 hours post injection onwards. To allow comparison between the different time points, images are shown at the mid-plane cross-section through the tumor. The images are corrected for injected dose and decay, and represented as $\% 1 D / m I$. 


\section{Quantification of 89Zr-Cetuximab Uptake}

To quantify the uptake of ${ }^{89} \mathrm{Zr}$-cetuximab in the different tumor types, threedimensional regions of interest (3D-ROIs) were manually drawn over the heart (outflow area), lungs, liver, kidneys, muscle and tumors. Figure $3 \mathrm{~A}$ shows the ${ }^{89} \mathrm{Zr}$ cetuximab uptake in the different tumors at all investigated time points. The accumulation in the intermediate EGFR-expressing tumors (U-373 MG and HT-29) was significantly higher at all time points compared with the high (A-431) and low (T-47D) EGFRexpressing tumors. Starting from $48 \mathrm{~h}$ p.i. the uptake levels differed significantly between all tumor types (i.e. uptake U-373 MG > HT-29 > A-431 > T-47D). Uptake in the $\mathrm{U}-373 \mathrm{MG}$ and HT-29 rose significantly until 48 and $24 \mathrm{~h}$ p.i. respectively, and demonstrated a plateau at about $9 \% \mathrm{ID} / \mathrm{ml}$ and $7 \% \mathrm{ID} / \mathrm{ml}$ respectively. For both tumor types, a $120 \mathrm{~h}$ p.i.time point was available, but tracer accumulation did not differ significantly from the $96 \mathrm{~h}$ p.i. time point. A similar uptake pattern was found for the A-431 tumors, although stable accumulation around $4 \% \mathrm{ID} / \mathrm{ml}$ was seen from $24 \mathrm{~h}$ p.i. onwards. The low EGFR-expressing T-47D tumors showed no significant increase of ${ }^{89} \mathrm{Zr}$-cetuximab uptake with time (around $1 \% \mathrm{ID} / \mathrm{ml}$ ).

Blood activity was measured using an ROI drawn on the outflow area of the heart. The

${ }^{89} \mathrm{Zr}$-cetuximab uptake in the blood showed a clearance pattern as depicted in figure $3 \mathrm{~B}$, starting from about $18 \% \mathrm{ID} / \mathrm{ml}$ (at $1 \mathrm{~h}$ p.i.) to $3 \% \mathrm{ID} / \mathrm{ml}(72-120 \mathrm{~h} \mathrm{p.i.).} \mathrm{This} \mathrm{uptake}$ was not influenced by the different tumor types expressing varying EGFR expression levels. We also investigated whether ${ }^{89} \mathrm{Zr}$-cetuximab uptake in normal tissues was dependent on tumor type; to this end, we quantified the uptake of ${ }^{89} \mathrm{Zr}$-cetuximab in several normal tissue regions using the $96 \mathrm{~h}$ p.i. PET images (figure 4), since stable tumor uptake was found at that time point. Uptake in normal tissues was relatively low compared with the tumor accumulation. Of the investigated tissues, liver and kidney uptake was highest, since those are the antibody clearance organs (especially the liver). Small, but not significant, pharmacokinetic differences in normal tissue uptake between animals bearing different tumor types were found, indicating that the normal tissue uptake was not affected by differences in tumor uptake and vice versa. 
A

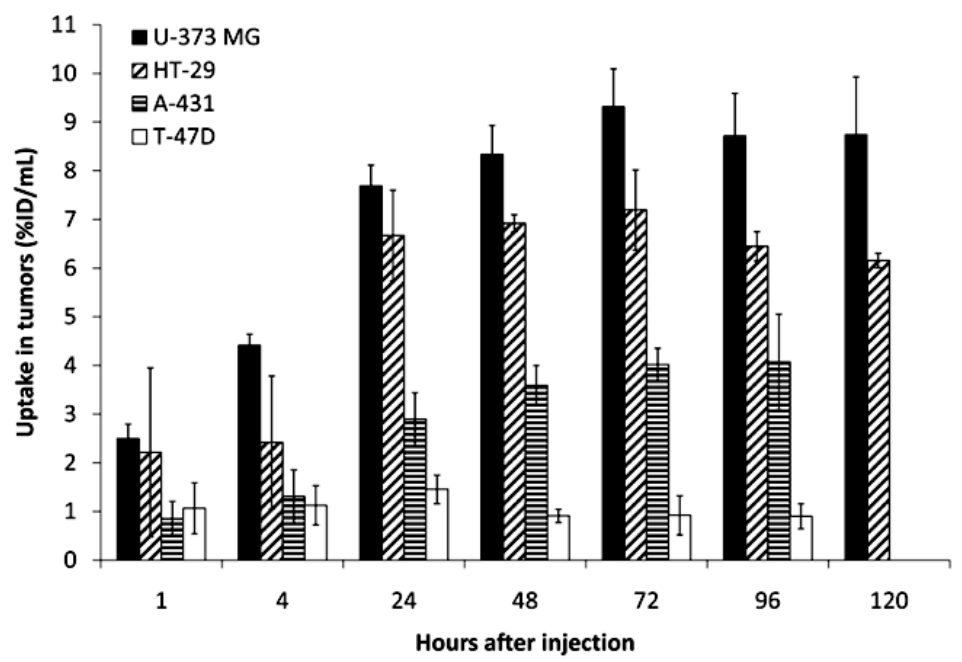

B

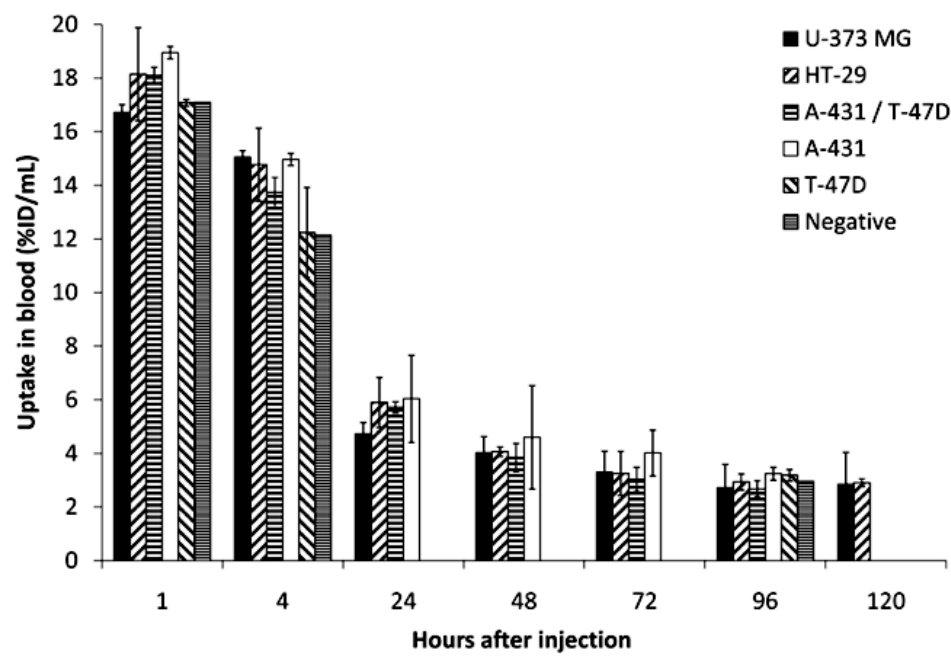

Figure 3

Quantification of ${ }^{89}$ Zr-cetuximab uptake from 1 to 120 hours post injection (p.i.). (A) Mean tumor uptake data for U-373 MG $(n=4), H T-29(n=4), A-431(n=6)$ and T-47D $(n=5)$ are shown and data are expressed as mean \pm standard deviation. For the U-373 MG and HT-29 tumors, images were also available at 120 hours p.i. All data were corrected for decay and injected dose of ${ }^{89} \mathrm{Zr}$ and are expressed as $\% I D / m I$. (B) Mean blood uptake as measured in the outflow area of the heart. Data are represented as mean \pm standard deviation for at least two independent mice. For comparison reasons, a non tumor-bearing mouse was included in the analysis. 


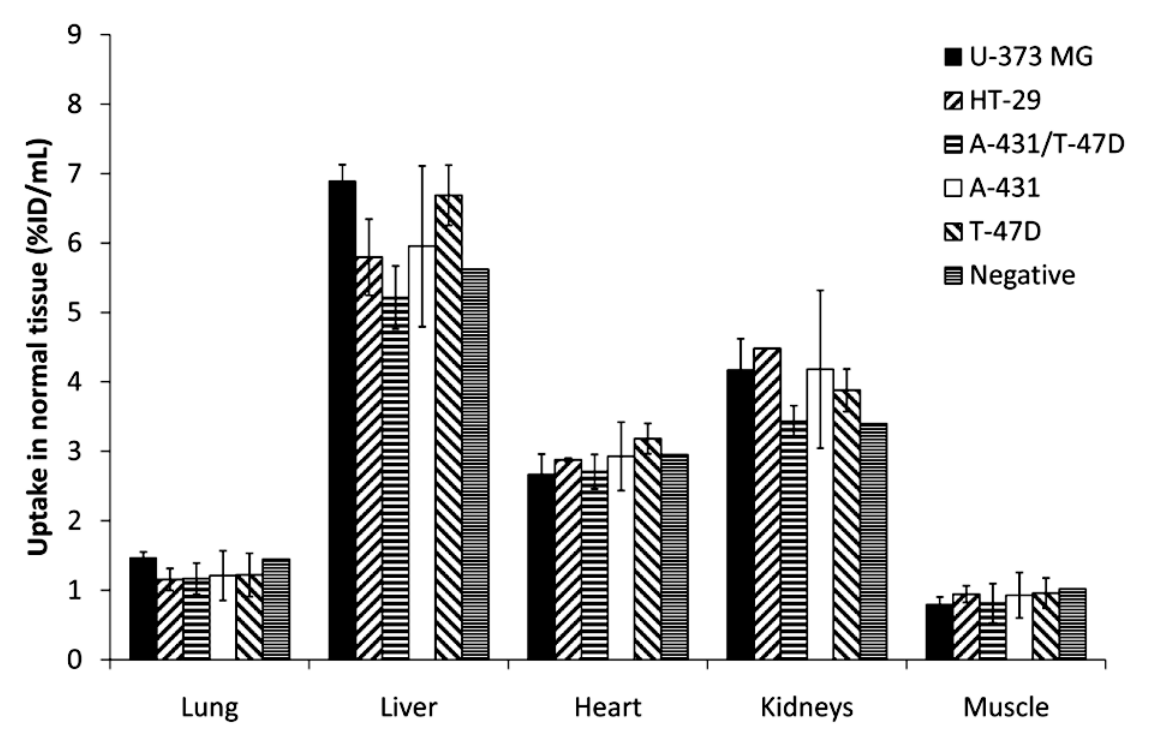

\section{Figure 4}

Normal tissue quantification of ${ }^{89} \mathrm{Zr}$-cetuximab uptake at 96 hours post injection from animals bearing U-373 MG ( $n=2), H T-29(n=2), A-431 / T-47 D(n=3), A-431(n=3), T-47 D(n=2)$ and negative $(n=1)$ tumor types. The data for the tumor-bearing mice are represented as mean uptake \pm standard deviation. All data were corrected for decay and injected dose of ${ }^{89} \mathrm{Zr}$, and are expressed as $\% I D / m l$.

\section{Tumor-to-Blood Ratios}

To assess the imaging potential of ${ }^{89} \mathrm{Zr}$-cetuximab, the tumor-to-blood activity ratios of the tracer were assessed. As shown in figure 3, an increasing tumor and a decreasing heart uptake was found, resulting in accumulating T/B ratios with time for the EGFRpositive tumors (figure 5). The mean tumor-to-mean blood (mean T/B) ratios are shown in figure $5 \mathrm{~A}$. After $24 \mathrm{~h}$ p.i., all mean T/B ratios were significantly different between all the investigated tumor types (i.e. ratio U-373 MG > HT-29 > A-431 > T-47D). The intermediate EGFR-expressing tumors (U-373 MG and HT-29) showed a significantly higher mean T/B ratio compared with the other tumors, reaching a plateau at 2 (HT-29) to 3 (U-373 MG) from $72 \mathrm{~h}$ p.i. on. In addition, the $120 \mathrm{~h}$ p.i.time point showed no increase in mean T/B ratio for those tumor types. The mean T/B ratios for the A-431 tumors increased until $48 \mathrm{~h}$ p.i. and demonstrated a plateau at 1.5. At no point did the mean T/B ratio of the T-47D tumors reach above unity. 


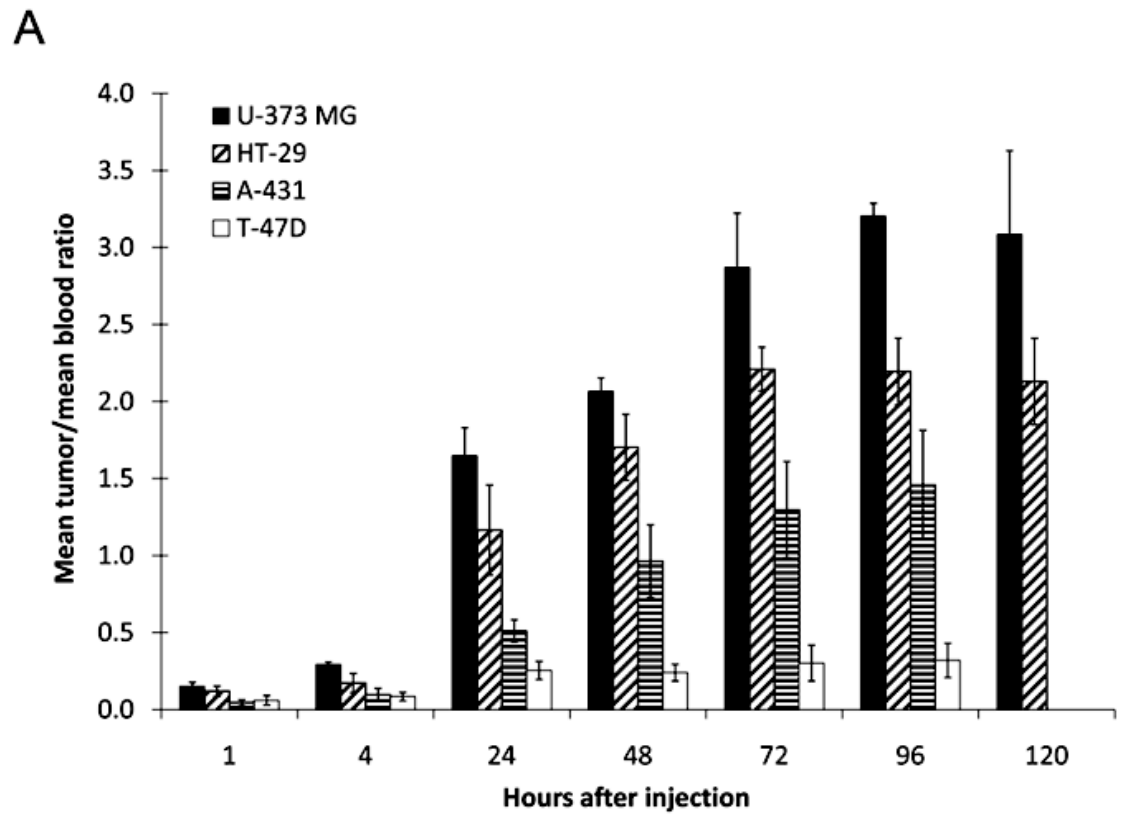

B

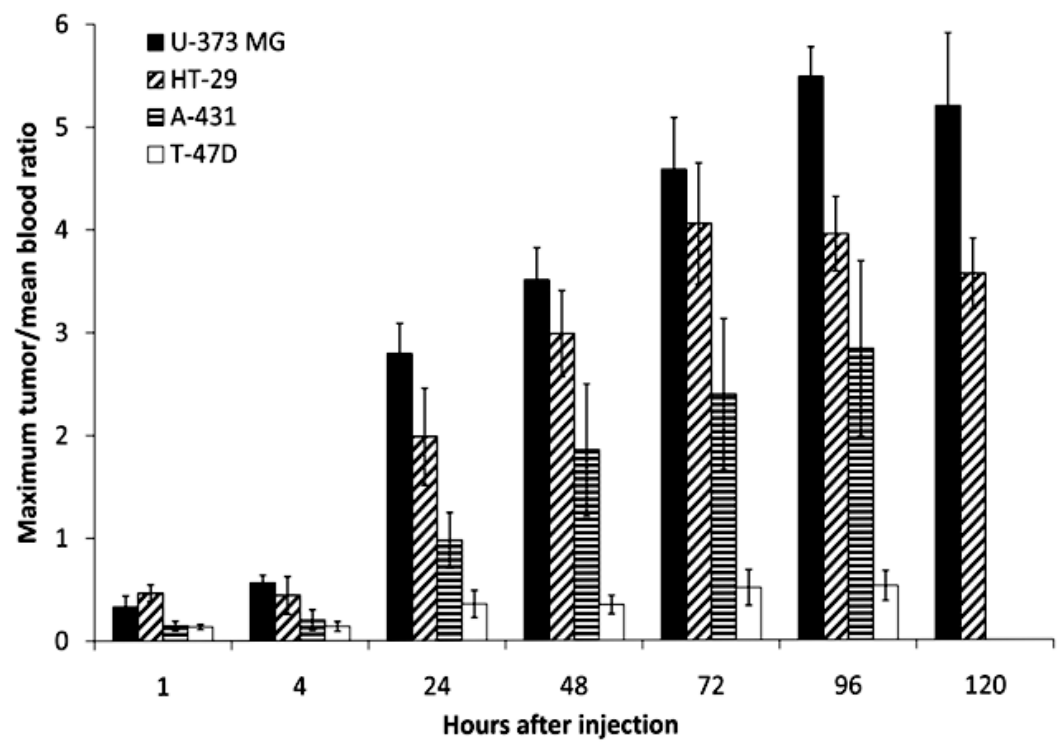

Figure 5

Tumor-to-blood ratios of ${ }^{89} \mathrm{Zr}$-cetuximab uptake from 1 to 120 hours post injection. The ratios represent the mean (A) or maximum (B) tumor uptake divided by the mean heart uptake. The data for U-373 MG (n=4), HT-29 (n=4), A-431 (n=6) and T-47D (n=5) are expressed as mean \pm standard deviation. 
Since tumors are known to be heterogeneous and the levels of heterogeneity between tumor types can differ, the mean and maximum uptake (i.e. the image voxel with the highest uptake) can vary considerably between different tumor types. Therefore, the maximum tumor uptake to mean blood $(\max T / B)$ ratios were also quantified (figure $5 \mathrm{~B})$. However, a similar pattern of ${ }^{89} \mathrm{Zr}$-cetuximab uptake was found with the mean tumor-to-mean blood uptake ratios, although the max T/B ratios were higher for the EGFR-positive tumors. The max T/B ratio of the T-47D tumors also never reached above unity. Differences in tumor growth during the experiment between the tumors with varying EGFR expression could influence the mean and max T/B ratios. However, the growth rate of the different tumor types during the experiment was comparable, indicating that the T/B ratios were largely independent of the tumor growth. The volume at the end of the experiment relative to day 0 for $U-373$ MG was: $127 \pm 33 \%$, A431: $141 \pm 22 \%$, HT-29: $142 \pm 17 \%$ and T-47D: $132 \pm 24 \%$.

\section{Gamma-counting Validation}

To validate the PET images and pharmacokinetics of ${ }^{89} \mathrm{Zr}$-cetuximab uptake, we performed gamma-counting on ex vivo normal and tumor tissue. Our results demonstrated that the U-373 MG tumors had the highest average uptake, followed by the HT-29, A-431; the lowest uptake was found in the T-47D tumors (figure 6A). Uptake in several selected normal tissues was available for A-431, T-47D, A-431/T-47D mixed and non tumor-bearing mice (figure $6 \mathrm{~B}$ ). No significant differences were found in normal tissue uptake between the mice bearing different tumor types. The uptake patterns, assessed using gamma-counting, were similar to the uptake obtained on the PET images. A full biodistribution was performed on the HT-29 tumor bearing mice (figure 6C). Uptake of all selected normal tissues was clearly lower compared with the HT-29 tumor uptake. The highest uptake (i.e. uptake $>3 \%$ ID/g) was found in the skin, salivary glands, liver, spleen and bladder, while other normal tissues including stomach, pancreas, ileum, colon, muscles, cerebrum, cerebellum and medulla oblongata showed low uptake $(<1 \%$ ID/g).

\section{Quantification of In vivo EGFR Expression}

Several micro-environmental parameters can influence in vivo EGFR expression, resulting in a difference between the in vivo and the in vitro tissue culture EGFR expression. We therefore assessed both the in vivo and in vitro EGFR expression using Western blotting. Figure 7A (upper panel) depicts representative Western blots of the different tumors, while in vitro samples are shown in the lower panel. Densitometric quantification of the in vivo Western blots is shown in figure 7B. The in vivo and in vitro EGFR expression was comparable, showing the A-431 tumors as high, U-373 MG and HT-29 as intermediate and T-47D as low EGFR-expressing cell lines. The A-431 expresses in vivo EGFR significantly higher compared to the other tumor types (U373-MG: $p=$ 
0.0002, HT-29: $p=0.0040$, T-47D: $p=0.0002)$. No significant differences were found between the U-373 MG and HT-29 tumor types ( $p=0.0902)$, while the T-47D was significantly lower compared to the other tumor types (U373-MG: $p=0.0024$, HT-29: $p=$ 0.0015, A-431: $p=0.002$ ).

\section{DISCUSSION}

Little is known about patient-specific tumor uptake and the relationship between dosage and efficacy of the mAb cetuximab, for which a disparity between EGFR expression levels and cetuximab treatment efficiency has often been reported ${ }^{14,15,17}$. To increase knowledge of the clinical use of cetuximab as a targeting agent, it is necessary to be able to monitor in vivo cetuximab uptake in a non-invasive manner. To address this, we developed a cetuximab-based probe for PET imaging. Here we report the in vivo validationof the ${ }^{89} \mathrm{Zr}$-labeled cetuximab imaging probe. The positron emitter ${ }^{89} \mathrm{Zr}$ was chosen given its ideal characteristics for usage with antibodies ${ }^{27,28}$. Like ${ }^{68} \mathrm{Ga}$ and ${ }^{64} \mathrm{Cu}$, ${ }^{89} \mathrm{Zrdemonstrates}$ residualization, meaning that these radionuclidesbecome trapped in lysosomes when the antibody internalizes and degrades ${ }^{27-29}$. Furthermore, ${ }^{89} \mathrm{Zr}$ shows a much longer physical half-life ( $t_{1 / 2}=78$ hour) than other residualizing radionuclides. Antibodies show generally slow pharmacokinetics and need several days to plateau in the target organ. Therefore, ${ }^{89} \mathrm{Zr}$ is the better-suited radionuclide for combination with intact antibodies (like cetuximab). To assess the pharmacokinetics of cetuximab noninvasively in order to increase current knowledge in clinical practice, using cetuximab itself as an imaging probe is the obvious solution.

To assess the pharmacokinetics of ${ }^{89} \mathrm{Zr}$-cetuximab, we performed PET imaging at several time points post injection and found a gradual increase in uptake in the high and intermediate EGFR-expressing tumors (figures 2 and $3 \mathrm{~A}$ ). The corresponding blood uptake decreased with time (figure 2 and $3 \mathrm{~B}$ ), resulting in high tumor-to-blood ratios (figure 5 ) at the plateau phase of cetuximab uptake ( 72 to 96 hours post injection). On the other hand, normal tissue uptake at similar time points was significantly lower (figure 4). Therefore, the tumor-to-blood ratios (maximum T/B from 3 to 5 ) attained are sufficient for imaging purposes. Our PET results were confirmed by ex vivo biodistribution studies assessed with gamma-counting. Again, uptake in the intermediate and high EGFR-expressing tumors was significantly higher compared with the surrounding normal tissues (figure 6). Although the gamma-counting data (in \%ID/g) demonstrated a similar trend to the quantified microPET data (in \%ID/ml), the absolute values of the gamma-counting were higher. This can be explained by the partial volume effects of the microPET scanner. 
To be able to predict which patients might benefit from cetuximab treatment, in vivo EGFR expression levels must be assessed. Therefore, we investigated whether the in vivo cetuximab uptake is associated with the EGFR expression levels. Other investigators reported the use of ${ }^{64} \mathrm{CU}$-DOTA-cetuximab for PET imaging, showing prominent uptake in EGFR-expressing tumors but low accumulation in EGFR-negative tumors ${ }^{22,24}$. A linear correlation between the \%ID/g values (measured by ${ }^{64} \mathrm{Cu}$ labeled cetuximab PET) and EGFR expression levels (assessed with Western blotting) was found.However, due to the limiting half-life of the isotope ${ }^{64} \mathrm{Cu}$, imaging was only possible until 48 hours p.i.. Also, the immunoreactive fraction of ${ }^{64} \mathrm{Cu}$-labeled cetuximab was significantly lower (about $63 \%{ }^{22}$ ) compared to ${ }^{89} \mathrm{Zr}$-labeled cetuximab (about $95 \%{ }^{28}$ ). Another study, with the murine counterpart of cetuximab (mC225) labeled with Indium111 , reported a correlation between mAb uptake and EGFR expression ${ }^{25}$. However, the aim of their study was not to evaluate this correlation, and the authors themselves state that the observed results should be compared cautiously, due to large differences in injected mAb concentration and image time points. Another explanation for these contradictory results can be the differences in tumor volume. Previous studies of De Bree et $a l^{34}$ and others showed that tumor volume largely determines antibody uptake, most likely related to blood supply, interstitial pressure and transport distance from the interstitium. To mimic a clinical situation more closely we used larger tumors $\left(\approx 400 \mathrm{~mm}^{3}\right)$ that displayed a relatively lower uptake of ${ }^{89} \mathrm{Zr}$-cetuximab compared with other studies with smaller tumors ${ }^{22,25}$. However, as shown in figure $5 B$ the maximum uptake voxel in the tumors ismuch higher $(\approx 20 \% \mathrm{ID} / \mathrm{g})$ and comparable with the uptake in the other studies.

Our results demonstrated a large disparity between the uptake of the antibody and the in vivo EGFR expression. Quantification of the PET images indicated that the uptake of the intermediate EGFR-expressing cell lines (U-373 MG \& HT-29) was approximately two times higher than the uptake in the high-expressing cells (A-431) (figure 3). However, Western blot quantification on ex vivo tumor material using anti-EGFR mAbs confirmed that EGFR expression of the A-431 tumors was higher than the U-373 MG and HT-29 tumors (figure 7). We confirmed the tumor EGFR expression by comparison with in vitro EGFR expression in cells and found similar results, indicating that the EGFR expression levels did not change in the tumor. We considered the possibility that differences in in vivo tumor growth could have affected the tumor uptake of the various xenograft lines; however, the growth was comparable between the various xenograft lines.

Tumors originating from different cell lines have (besides EGFR expression) other physiologic effects, like inadequate vasculature, differences in capillary permeability perfusion, and differences in necrosis. All these factors can play an important role in in vivo drug delivery ${ }^{35,36}$. Also, cancer cells can bypass the dependency of EGFR for cell 
growth and survival by altering EGFR levels through promoting EGFR ubiquitination and degradation, resulting in overall cetuximab resistance ${ }^{36}$. Therefore, it should be noted that if cetuximab is not able to reach the complete tumor, no therapeutic effect can be expected in these regions. However, if cetuximab is able to reach the complete tumor, a therapeutic effect may not necessarilyoccur given the redundancy in the EGFR pathway.

Taken together, the relationship between EGFR expression levels, cetuximab binding and, ultimately, clinical outcome is complex. In vitro there is a strong relationship between cetuximab binding and EGFR expression levels ${ }^{16}$. Also, high levels of EGFR expression are correlated with poor clinical outcomes ${ }^{5}$. However, cetuximab's treatment efficacy is not correlated with EGFR expression ${ }^{14,15,17}$. Our results show that cetuximab uptake is not only dependent on EGFR expression levels, revealing additional mechanisms influencing tumor delivery of cetuximab. These mechanisms may partially explain why expression alone may not be sufficient to explain therapy effects. Possible explanations for the mismatch may include inadequate vasculature and perfusion, resulting in a heterogeneous and limited cetuximab uptake, even upon treatment at high $m A b$ dose. A future quantification of the results, assessing differences in vasculature, perfusion, hypoxia and proliferation, could explain why the 'reachability' in the xenografts is disparate.

The chelate labeling of ${ }^{89} \mathrm{Zr}$ with cetuximab is very stable: indeed, it can be produced under good manufacturing procedure (GMP) settings, making it an ideal candidate for a fast translation to the clinic ${ }^{27}$. In future clinical trials, ${ }^{89} \mathrm{Zr}$-labeled cetuximab may be used to non-invasively examine the uptake of cetuximab into the tumor, selecting patients who can potentially benefit from cetuximab treatment. Sequential imaging during treatment can also reveal microenvironmental changes, influencing cetuximab perfusion and uptake. Based on these images, treatment can be monitored and dosage adjusted, making individualized treatment of cetuximab a possibility.

\section{CONCLUSION}

This study has demonstrated that cetuximab uptake in tumors can be assessed by PET imaging using ${ }^{89} \mathrm{Zr}$-labeled cetuximab. This probe can thus potentially be used to evaluate the biologic and pharmacokinetic clinical effects of EGFR inhibitors, both cetuximab and others. A disparity was found between cetuximab uptake and in vivo EGFR expression; the imaging therefore represents cetuximab uptake instead of a quantification of EGFR expression levels. More generally, the results reveal additional mechanisms that 
influence antibody uptake in tumors besides the expression levels of a biologic target in a tumor alone.

\section{ACKNOWLEDGEMENTS}

The authors would like to thank Nicole Bitsch for her technical assistance with the animal experiments. We acknowledge financial support from the Dutch Cancer Society (KWF Kankerbestrijding, Grant: UM2008-4210).

\section{REFERENCES}

1. Imai $\mathrm{K}$ and Takaoka $\mathrm{A}$, Comparing antibody and small-molecule therapies for cancer, Nat Rev Cancer 6 (9), 714-727 (2006).

2. Sebolt-Leopold JS and English JM, Mechanisms of drug inhibition of signalling molecules, Nature 441 (7092), 457-462 (2006).

3. Herbst RS, Review of epidermal growth factor receptor biology, Int J Radiat Oncol Biol Phys 59 (2 Suppl), 21-26 (2004).

4. Mendelsohn J, Targeting the epidermal growth factor receptor for cancer therapy, J Clin Oncol 20 (18 Suppl), 1S-13S (2002).

5. Akimoto T, Hunter NR, Buchmiller L, et al., Inverse relationship between epidermal growth factor receptor expression and radiocurability of murine carcinomas, Clin Cancer Res 5 (10), 2884-2890 (1999).

6. Eriksen JG, Steiniche T and Overgaard J, The influence of epidermal growth factor receptor and tumor differentiation on the response to accelerated radiotherapy of squamous cell carcinomas of the head and neck in the randomized DAHANCA 6 and 7 study, Radiother Oncol 74 (2), 93-100 (2005).

7. Herbst RS and Shin DM, Monoclonal antibodies to target epidermal growth factor receptor-positive tumors: a new paradigm for cancer therapy, Cancer 94 (5), 1593-1611 (2002).

8. Baselga J and Arteaga $\mathrm{CL}$, Critical update and emerging trends in epidermal growth factor receptor targeting in cancer, J Clin Oncol 23 (11), 2445-2459 (2005).

9. Huang SM, Bock JM and Harari PM, Epidermal growth factor receptor blockade with C225 modulates proliferation, apoptosis, and radiosensitivity in squamous cell carcinomas of the head and neck, Cancer Res 59 (8), 1935-1940 (1999).

10. Huang SM and Harari PM, Modulation of radiation response after epidermal growth factor receptor blockade in squamous cell carcinomas: inhibition of damage repair, cell cycle kinetics, and tumor angiogenesis, Clin Cancer Res 6 (6), 2166-2174 (2000).

11. Bonner JA, Harari PM, Giralt J, et al., Radiotherapy plus Cetuximab for Squamous-Cell Carcinoma of the Head and Neck, N Engl J Med 354 (6), 567-578 (2006).

12. Lammering $\mathrm{G}$, Molecular predictor and promising target: will EGFR now become a star in radiotherapy?,Radiother Oncol 74 (2), 89-91 (2005). 
13. Erbitux (Cetuximab) in the treatment of metastatic colorectal cancer. Company Report Merck KGaA.

14. Hebbar M, Wacrenier A, Desauw $C$, et al., Lack of usefulness of epidermal growth factor receptor expression determination for cetuximab therapy in patients with colorectal cancer, Anticancer Drugs 17 (7), 855-857 (2006).

15. Lenz HJ, Van Cutsem E, Khambata-Ford S, et al., Multicenter phase II and translational study of cetuximab in metastatic colorectal carcinoma refractory to irinotecan, oxaliplatin, and fluoropyrimidines, J Clin Oncol 24 (30), 4914-4921 (2006).

16. Aerts HJ, Dubois L, Hackeng TM, et al., Development and evaluation of a cetuximab-based imaging probe to target EGFR and EGFRvIII, Radiother Oncol 83 (3), 326-332 (2007).

17. Chung KY, Shia J, Kemeny NE, et al., Cetuximab shows activity in colorectal cancer patients with tumors that do not express the epidermal growth factor receptor by immunohistochemistry, J Clin Oncol 23 (9), 1803-1810 (2005).

18. Heidary N, Naik H and Burgin S, Chemotherapeutic agents and the skin: An update, J Am Acad Dermatol 58 (4), 545-570 (2008).

19. Divgi $C R$, Welt $S$, Kris $M$, et al., Phase I and imaging trial of indium 111-labeled antiepidermal growth factor receptor monoclonal antibody 225 in patients with squamous cell lung carcinoma, J Natl Cancer Inst 83 (2), 97-104 (1991).

20. van Dongen GA, Visser GW, Lub-de Hooge MN, et al., Immuno-PET: A Navigator in Monoclonal Antibody Development and Applications, Oncologist 12 (12), 1379-1389 (2007).

21. Schechter NR, Yang DJ, Azhdarinia A, et al., Assessment of epidermal growth factor receptor with 99mTc-ethylenedicysteine-C225 monoclonal antibody, Anticancer Drugs 14 (1), 49-56 (2003).

22. Cai W, Chen K, He L, et al., Quantitative PET of EGFR expression in xenograft-bearing mice using (64)Cu-labeled cetuximab, a chimeric anti-EGFR monoclonal antibody, Eur J Nucl Med Mol Imaging (2007).

23. Schechter NR, Wendt RE, 3rd, Yang DJ, et al., Radiation dosimetry of 99mTc-labeled C225 in patients with squamous cell carcinoma of the head and neck, J Nucl Med 45 (10), 16831687 (2004).

24. Ping Li W, Meyer LA, Capretto DA, et al., Receptor-Binding, Biodistribution, and Metabolism Studies of (64)Cu-DOTA-Cetuximab, a PET-Imaging Agent for Epidermal Growth-Factor Receptor-Positive Tumors, Cancer Biother Radiopharm 23 (2), 158-171 (2008).

25. Goldenberg A, Masui H, Divgi C, et al., Imaging of human tumor xenografts with an indium111-labeled anti-epidermal growth factor receptor monoclonal antibody, J Natl Cancer Inst 81 (21), 1616-1625 (1989).

26. Verel I, Visser GW, Boellaard R, et al., Quantitative 89Zr immuno-PET for in vivo scouting of 90Y-labeled monoclonal antibodies in xenograft-bearing nude mice, J Nucl Med 44 (10), 1663-1670 (2003).

27. Verel I, Visser GW, Boellaard R, et al., 89Zr immuno-PET: comprehensive procedures for the production of $89 \mathrm{Zr}$-labeled monoclonal antibodies, J Nucl Med 44 (8), 1271-1281 (2003).

28. Perk LR, Visser GW, Vosjan MJ, et al., (89)Zr as a PET surrogate radioisotope for scouting biodistribution of the therapeutic radiometals (90)Y and (177)Lu in tumor-bearing nude mice after coupling to the internalizing antibody cetuximab, J Nucl Med 46 (11), 1898-1906 (2005). 
29. Verel I, Visser GW, Boerman OC, et al., Long-lived positron emitters zirconium-89 and iodine-124 for scouting of therapeutic radioimmunoconjugates with PET, Cancer Biother Radiopharm 18 (4), 655-661 (2003).

30. Verel I, Visser GW, Vosjan MJ, et al., High-quality 124I-labelled monoclonal antibodies for use as PET scouting agents prior to 131l-radioimmunotherapy, Eur J Nucl Med Mol Imaging 31 (12), 1645-1652 (2004).

31. Nagengast WB, de Vries EG, Hospers GA, et al., In vivo VEGF imaging with radiolabeled bevacizumab in a human ovarian tumor xenograft, J Nucl Med 48 (8), 1313-1319 (2007).

32. Borjesson PK, Jauw YW, Boellaard R, et al., Performance of immuno-positron emission tomography with zirconium-89-labeled chimeric monoclonal antibody U36 in the detection of lymph node metastases in head and neck cancer patients, Clin Cancer Res 12 (7 Pt 1), 2133-2140 (2006).

33. Perk LR, Visser OJ, Stigter-van Walsum M, et al., Preparation and evaluation of (89)ZrZevalin for monitoring of (90)Y-Zevalin biodistribution with positron emission tomography, Eur J Nucl Med Mol Imaging 33 (11), 1337-1345 (2006).

34. de Bree R, Kuik DJ, Quak JJ, et al., The impact of tumour volume and other characteristics on uptake of radiolabelled monoclonal antibodies in tumour tissue of head and neck cancer patients, Eur J Nucl Med 25 (11), 1562-1565 (1998).

35. Viloria-Petit A, Crombet $T$, Jothy $S$, et al., Acquired resistance to the antitumor effect of epidermal growth factor receptor-blocking antibodies in vivo: a role for altered tumor angiogenesis, Cancer Res 61 (13), 5090-5101 (2001).

36. Lu Y, Li X, Liang K, et al., Epidermal growth factor receptor (EGFR) ubiquitination as a mechanism of acquired resistance escaping treatment by the anti-EGFR monoclonal antibody cetuximab, Cancer Res 67 (17), 8240-8247 (2007). 



\section{CHAPTER}

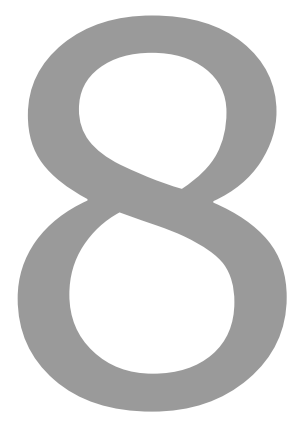

\section{Imaging of drug uptake: \\ Clinical in vivo imaging of Cetuximab}

Manuscript in preparation

Non invasive PET imaging of Cetuximab-Zirconium-89 uptake: A phase I trial Judith van Loon, Hugo J.W.L. Aerts, Marco H.M. Janssen, Michel Öllers, Ludwig Dubois, AnneMarie C. Dingemans, A. van Belle, M. Hochstenbag, R. Lalisang, Erik Vegt, Ronald Boellaard, Guus A. van Dongen, Dirk De Ruysscher, Philippe Lambin 


\section{ABSTRACT}

\section{Background and Purpose}

Non invasive PET imaging of cetuximab uptake could help to select patients with the highest chance to benefit from this treatment. Cetuximab labelled with Zirconium-89 is a promising new conjugate to determine tumour uptake of cetuximab, which has been tested in pre-clinical animal models with excellent results (Aerts et al, J Nucl Med 2009). In this phase I trial (NCT00691548), we aimed to determine the toxicity of ${ }^{89} \mathrm{Zr}$ cetuximab in incurable cancer patients, with tumour visualization as a secondary aim.

\section{Material and Methods}

The safety of two different dose schedules was tested. In step 1, the toxicity of two consecutive low radioactivity doses of ${ }^{89} \mathrm{Zr}$-cetuximab (60 MBq) was investigated. In step 2, the toxicity of a single larger dose (120 MBq) was investigated. Labelled cetuximab administration was preceded by a standard loading dose of unlabelled cetuximab. Toxicity was scored twice weekly up to 14 days after injection, according to the CTCAE 3.0 scoring system and with blood tests for haematological and liver/kidney function. Total body PET-CT scans were acquired on day 4, 5 and 6 (step 1) and 5, 6, 7 (step 2) post injection. Images were analyzed using dedicated software.

\section{Results}

Seven patients with a histology proven solid tumour without curative treatment options were included. One patient was excluded before administration of the studied drug because of hypertension during the administration of unlabelled cetuximab. Six patients had NSCLC, whereas 1 patient had a tonsillar carcinoma. Five of six patients who completed the study experienced skin toxicity. No additional toxicity was associated with the administration of ${ }^{89} \mathrm{Zr}$-cetuximab. The maximal tumour to blood ratio (TBR) observed was 1.6. In general, the most optimal TBR was found at later imaging time points (day 6 and day 7).

\section{Conclusions}

No additional toxicity was observed for ${ }^{89} \mathrm{Zr}$-cetuximab administration. ${ }^{89} \mathrm{Zr}$-cetuximab is thus safe to be implemented in ummuno-PET studies guiding cetuximab therapy. 


\section{INTRODUCTION}

Monoclonal antibodies are increasingly used in oncology as means to specifically target receptors at the surface of tumour cells that are associated with tumour aggressiveness, such as proliferation, hypoxia and angiogenesis. Cetuximab is a monoclonal antibody that blocks the Epidermal Growth Factor Receptor (EGFR). The EGFR is a transmembrane glycoprotein which is commonly expressed in many normal human tissues. It is a member of the tyrosine kinase family of growth factor receptors and is overexpressed in many human malignancies ${ }^{1,2}$. EGFR is activated upon ligand binding to the extracellular domain, which results in the induction of a signal transduction pathway that cascades many cellular operations concerning cell growth and division. EGFR activation and overexpression appear to be important tumour cell mechanisms in the development of resistance to radiation and chemotherapy in epithelial cancers, with decreased rates of local tumour control and survival ${ }^{3-7}$.

Cetuximab specifically binds to the EGFR with high affinity, internalising the receptor and thus effectively blocking ligand-induced EGFR phosphorylation. It is a promising molecule to combine with radiotherapy alone or with concurrent chemo-radiation, as it has shown to improve local tumour control without enhancing the toxicity of radiotherapy or chemotherapy ${ }^{8,9}$. It plays currently a role as part of the standard treatment for a variety of cancers, including colorectal cancer and squamous cell carcinoma of the head and neck. The selection of patients with the highest chance of benefit from cetuximab treatment is of obvious relevance, also in view of its high costs. As no direct relationship has been observed between EGFR expression determined with immunohistochemistry and response to cetuximab treatment, EGFR status of the primary tumour cannot be used as a selection criterion. As the expression of EGFR can be induced by radiation and chemotherapy, it is possible that an initially EGFR negative tumour may become positive early during therapy, thereby still benefiting from cetuximab treatment ${ }^{10-12}$. On the other hand, EGFR expressing tumours will not respond to cetuximab when cetuximab does not reach therapeutic concentrations in the tumour because of e.g. interstitial pressure and vascular perfusion changes in the microenvironment of the tumour or the surrounding tissues. In this regard, non-invasive visualization and quantification of tumour uptake of cetuximab with positron emission tomography (PET) before and during therapy could contribute significantly to patient selection and determination of the needed dosage ${ }^{13}$.

As the biologic half-life of cetuximab in the blood is $70-100$ hours, a positron emitter with a long half-life is needed to visualize its uptake. Zirconium-89 $\left({ }^{89} \mathrm{Zr}\right)$, a positron emitter with a half life of approximately 78 hours, can successfully be labelled to cetuximab and has shown excellent results in preclinical animal models ${ }^{14}$. In the preclinical study, tumour cell lines with varying EGFR expression levels were used for in 
vivo imaging experiments. PET with ${ }^{89} \mathrm{Zr}$-labeled cetuximab was performed on tumourbearing NMRI-nu mice at multiple time points after injection and uptake was quantified within regions of interest on selected tissues. Uptake of ${ }^{89} \mathrm{Zr}$-labeled cetuximab was demonstrated in the EGFR-positive tumours. Furthermore, it was shown that indeed, levels of EGFR expression did not correlate with ${ }^{89} \mathrm{Zr}$-cetuximab uptake, implying that pharmacokinetic and -dynamic factors strongly influence the cetuximab distribution in the tumour ${ }^{14}$.

Here, we report the results of a phase I study to determine toxicity of Zirconium-89 labelled cetuximab in incurable cancer patients, with tumour visualisation as a secondary end point.

\section{METHODS}

\section{Patient selection}

Patients with a histologically or cytologically confirmed solid cancer at any site without curative treatment options were eligible. Inclusion criteria were as follows: WHO performance status 0 to 2; adequate bone marrow, hepatic and renal function (calculated creatinin clearance at least $60 \mathrm{ml} / \mathrm{min}$ ); life expectancy $>3$ months and written informed consent to the study. Patients with a recent ( $<3$ months) myocardial infarction, uncontrolled infectious disease, pregnancy, previous administration of cetuximab or concurrent treatment with anti-cancer agents or radiotherapy were excluded. EGFR expression and mutation status as well as k-ras mutation were assessed for the primary tumour. The study protocol (NCT00691548) was approved by the medical ethical committee and the radiation safety committee (MECazM 083039.7 and 2008-8, respectively)

\section{Study design}

A two dose-step study design was used, graphically represented in Figure 1 . The study design anticipated on the future aim, which is to determine the tumour uptake of ${ }^{89} \mathrm{Zr}$ cetuximab before and during therapy. Therefore, in step 1, the toxicity of two consecutive low doses of ${ }^{89} \mathrm{Zr}$-cetuximab was investigated. A standard loading dose of 400 $\mathrm{mg} / \mathrm{m} 2$ of cetuximab was administered, followed by administration of cetuximab labelled ${ }^{89} \mathrm{Zr}(60 \mathrm{MBq})$ on day 0 . The second injection on day 14 followed consisted of a dose of $250 \mathrm{mg} / \mathrm{m} 2$ of cetuximab, followed by cetuximab labelled with $60 \mathrm{MBq}{ }^{89} \mathrm{Zr}$. 
Step 1

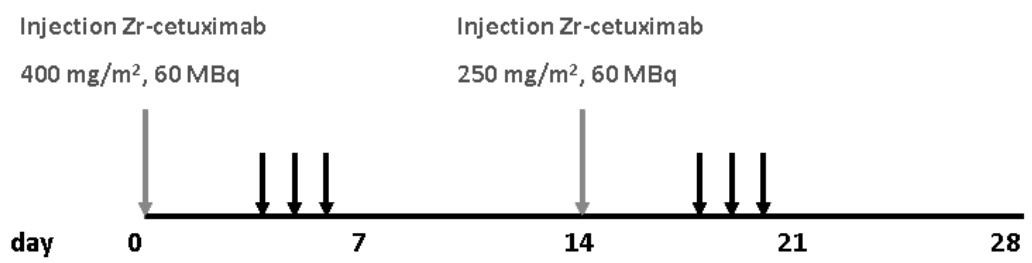

Step 2

Injection Zr-cetuximab

$400 \mathrm{mg} / \mathrm{m}^{2}, 120 \mathrm{MBq}$

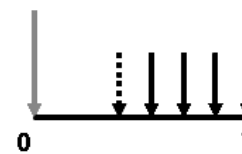

14

21

28

* days of scanning in step 2 depend on imaging results in step 1

\section{Figure 1 Timeline of the study}

A two dose-step study design was used. In step 1, the toxicity of two lower doses of ${ }^{89} \mathrm{Zr}$ cetuximab was investigated. In step 2, the toxicity of a single larger dose of cetuximab was tested. PET-CT imaging was performed at three consecutive days after administration, starting at day 4 in dose step $1 .{ }^{*}$ In step 2, PET-CT scans on day 3 and 7 after injection were optional and depended on the imaging results in step 1: If in step 1 two or more patients had a higher tumour to blood ratio (TBR) on day 4 then on day 5, an additional scan on day 3 would be performed. If in step 1 two or more patients had a higher TBR on day 6 then on day 5, an additional scan on day 7 would be performed

As a larger radioactive dose of ${ }^{89} \mathrm{Zr}$ is possibly needed to obtain the best image quality, the toxicity of a single larger dose was investigated in step 2. A loading dose of 400 $\mathrm{mg} / \mathrm{m}^{2}$ of cetuximab was administered in 3 patients, followed by administration of the cetuximab labelled with $120 \mathrm{MBq}{ }^{89} \mathrm{Zr}$.

In both steps, the ${ }^{89} \mathrm{Zr}$-labelled cetuximab was administered within one hour after the administration of the loading dose. Six patients were included, three in each dose step. If grade 2 or higher toxicity was observed related to the administration of the studied drug $\left({ }^{89} \mathrm{Zr}\right.$-labelled cetuximab) in any patient, 3 more patients were included in this dose step. When at maximum $1 / 6$ patients experienced grade 2 toxicity, the step was considered safe. 


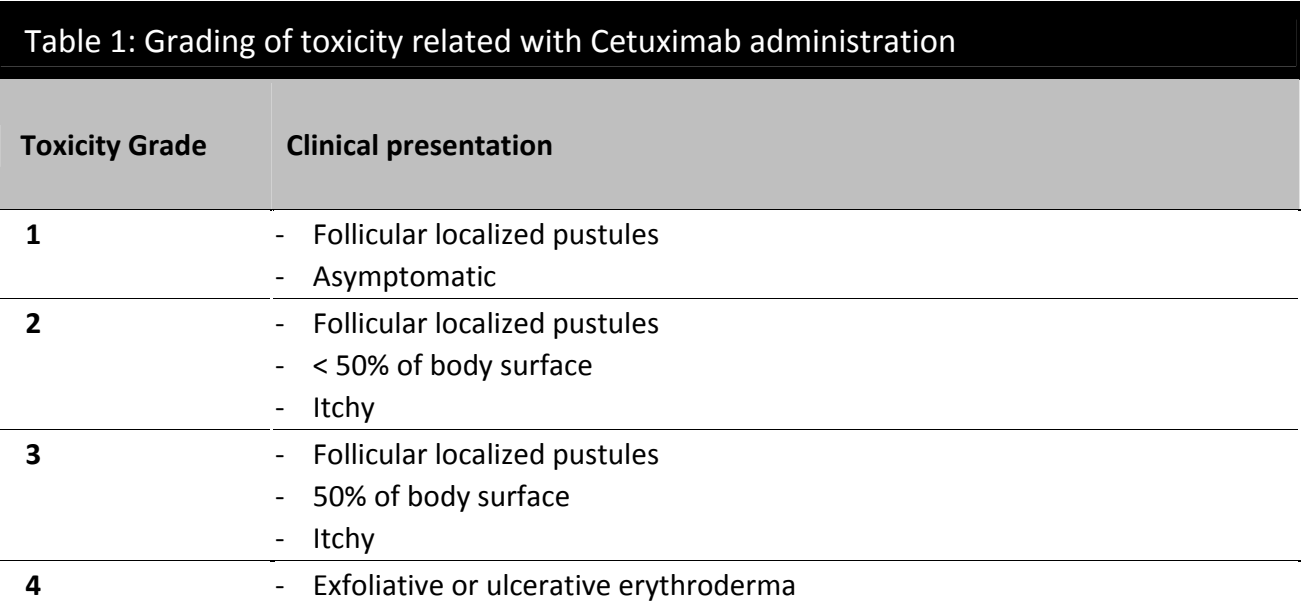

\section{Toxicity scoring}

Toxicity was assessed during treatment, and twice weekly thereafter until 14 days after the last injection, according to the CTCAE 3.0 scoring system. At baseline and on days 7 and 14 after injection, blood testing was performed for haematology (haemoglobin, hematocrite, mean cell volume, reticulocytes, white blood cell count and differentiation, platelets), kidney function (creatinin) and liver function (aspartate aminotransferase, alanine aminotransferase, alkalic phosphatase, $y$-GT, bilirubin). The most common side effect of cetuximab administration that was anticipated for was skin toxicity (acne form rash). The classification of the degree of skin toxicity according to the CTCAE is provided in Table $1^{15}$.

\section{Synthesis of ${ }^{89} \mathrm{Zr}$ labelled cetuximab}

${ }^{89} \mathrm{Zr}$ was produced by a $(p, n)$ reaction on natural ${ }^{89} \mathrm{Y}$ as described by Verel et al. ${ }^{16}$. Subsequently, labelling of ${ }^{89} \mathrm{Zr}$ to the mAb cetuximab was performed as previously reported ${ }^{17}$. Briefly, desferrioxamine B was succinylated (N-sucDf), temporarily filled with iron $\left(\mathrm{Fe}^{3+}\right)$, and coupled to cetuximab using a tetrafluorophenol-N-sucDf ester. The premodified mAb was purified on a PD10 column after removal of $\mathrm{Fe}^{3+}$ by transchelation to ethylenediaminetetraacetic acid. Approximately $1 \mathrm{~N}$-sucDf moiety was coupled per cetuximab, assessed by using ${ }^{59} \mathrm{Fe}$. Next, N-sucDf-cetuximab was labelled with ${ }^{89} \mathrm{Zr}$ in $0.5 \mathrm{M} \mathrm{N}$-(2-hydroxyethyl)piperazine-N9-(2-ethanesulfonic acid) (HEPES) buffer at $\mathrm{pH}$ 7.0 and again purified on a PD10 column to remove unbound ${ }^{89} \mathrm{Zr}$. ${ }^{89} \mathrm{Zr}$-cetuximab preparations contained $10 \mathrm{mg}{ }^{89} \mathrm{Zr}$-cetuximab in $5 \mathrm{mg} / \mathrm{ml}$ gentisic acid in $0.9 \% \mathrm{NaCl}$, containing 60 or $120 \mathrm{MBq}{ }^{89} \mathrm{Zr} .{ }^{89} \mathrm{Zr}$-cetuximab release criteria were: radiochemical pu- 
rity > 90\%; immunoreactivity $>70 \%$; $\mathrm{pH}=4.7-5.3$; sterile and free of endotoxins (European Pharmacopeia).

\section{PET-CT imaging}

Whole body PET-CT imaging was performed at day 4, 5 and 6 after injection in dose step 1. Imaging time points in step 2 were adapted based on the tumour to blood ratios at subsequent days in step 1. A list mode acquisition of the largest tumour site was carried out in addition to the whole body PET-CT. Furthermore, an optional ${ }^{18} \mathrm{~F}$ fluorodeoxyglucose (FDG-)PET scan was performed within the study period, at least 24 hours before or 10 days after the ${ }^{89} \mathrm{Zr}$-cetuximab administration. Images were acquired with the patient in supine position. CT and PET images were automatically registered based on mutual information and were subsequently fused using dedicated software (TrueD, version VC50, Siemens Medical, Erlangen, Germany). The tumour was delineated on the CT scan by the same observer (JVL). If available, FDG-PET information was used in addition to the CT. The maximal standardized uptake value (SUV $\left.\mathrm{V}_{\max }\right)$ and the location of the ${ }^{89} \mathrm{Zr}$-cetuximab uptake within the delineated tumour were determined using dedicated software (TrueD VC50, Siemens Medical, Erlangen, Germany). Tumour to blood ratios (TBRs) for ${ }^{89} \mathrm{Zr}$-cetuximab uptake were calculated at all time points by dividing the tumour SUV max $_{\text {m }}$ by the SUV $\mathrm{Smax}_{\max }$ in the aortic arch. For patients in whom an additional ${ }^{18} \mathrm{~F}$-FDG-PET scan was performed within one week before or after the ${ }^{89} \mathrm{Zr}$ cetuximab scan, the ${ }^{18} \mathrm{FDG}$ and ${ }^{89} \mathrm{Zr}$-cetuximab uptake was visually compared. The location of the FDG uptake was determined using automated SUV-thresholding with the threshold depending on the tumour-to-background signal ratio (SBR-method) ${ }^{18,19}$.

\section{RESULTS}

\section{Patient characteristics}

Seven patients, with a median age of 63 years (range: $53-75)$, were included. The third patient was excluded from the study before injection of the ${ }^{89} \mathrm{Zr}$-labelled cetuximab, because of malignant hypertension during the administration of the unlabelled cetuximab. The tension normalized within two hours of observation without further sequelae. Patient and tumour characteristics are shown in Table 2. Six patients had a non-small cell lung carcinoma (NSCLC) and 1 had a tonsillar carcinoma. All patients had previously undergone anti-cancer therapy. In one patient, EGFR expression and mutation of k-ras mutation status of the primary tumour could not be assessed due to insufficient quantity of the histological material. In the other patients, none of the primary tumours showed overexpression of EGFR or a mutation in de exons 18-21 of the EGFR gene. In one patient, the tumour showed a mutation in the k-ras gene. 
Table 2: Patient characteristics

\begin{tabular}{|c|c|c|c|c|c|c|c|c|c|c|c|}
\hline 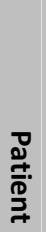 & $\underset{\substack{\text { Od } \\
0}}{D}$ & 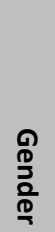 & 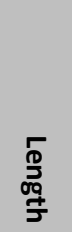 & 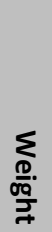 & 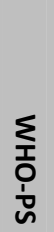 & 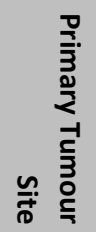 & $\begin{array}{l}\text { I } \\
\overline{5} \\
\frac{0}{0} \\
\frac{0}{0} \\
\text { gू }\end{array}$ & 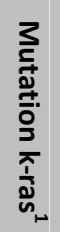 & 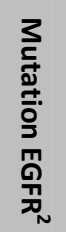 & 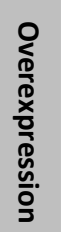 & 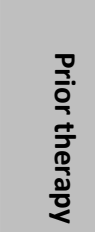 \\
\hline 1 & 72 & $M$ & 1.85 & 75 & 0 & Lung & SCC & NA & NA & NA & CTX \\
\hline 2 & 61 & $M$ & 1.76 & 82 & 1 & Lung & LCC & + & - & No & CTx, RT \\
\hline $3^{3}$ & 55 & $\mathrm{~F}$ & 1.56 & 61 & 0 & Lung & LCC & NA & NA & NA & CTX \\
\hline 4 & 75 & $M$ & 1.79 & 74 & 2 & Lung & $A C$ & - & - & No & CTX \\
\hline 5 & 53 & $M$ & 1.80 & 91 & 1 & Lung & SCC & - & - & No & CTX \\
\hline 6 & 63 & $M$ & 1.78 & 86 & 0 & Lung & $A C$ & - & - & No & CTX \\
\hline 7 & 63 & $M$ & 1.86 & 95 & 1 & Tonsil & SCC & - & - & No & $\mathrm{RT}, \mathrm{CT} \mathrm{x}$ \\
\hline
\end{tabular}

${ }^{1}$ k-ras: codons 12 and $13 ;^{2}$ EGFR: exons 18-21; ${ }^{3}$ Excluded patient: 55 year old female, NSCLC WHO-PS: World Health Organization Performance Status; $M=$ male, $F=$ female, SCC: squamous cell carcinoma; LCC: large cell carcinoma; AC: adenocarcinoma; NA = not assessable; CTx: Chemotherapy; RT: radiotherapy

Each patient received ${ }^{89} \mathrm{Zr}$-labelled cetuximab administration as planned. In two patients (patient 2 and 4) enrolled in the first dose step, however, the second series of PET-CT scans could not be completed due to pain and dyspnea attributable to disease progression.

\section{Toxicity}

An overview of the toxicity per patient is presented in Table 3. As mentioned before, patient 3 was prematurely excluded from the study due to malignant hypertension, unrelated to the study drug. For this reason, an extra patient was included in dose step 1. No toxicity other than skin rash was observed according to the CTCAE 3.0 criteria in the other three patients included in dose step 1 . The same held true for the three patients included in step 2, with again no toxicity other than skin toxicity. Of the six patients that received the full cetuximab administration, two experienced grade 1 , and 3 experienced grade 2 acne-form rash, which was treated with local (metronidazol cream) and systemic antibiotics (doxycyclin) ${ }^{15}$. No change in the baseline grade according to the CTCAE version 3.0 was observed for haematological, kidney and liver function. 


\section{Table 3: Toxicity scoring}

\begin{tabular}{|c|c|c|c|c|c|c|c|c|}
\hline 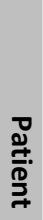 & 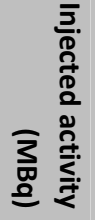 & 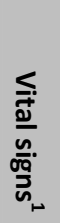 & 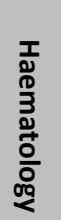 & 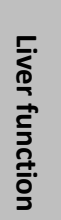 & 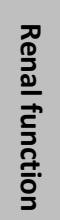 & 彦 & \begin{tabular}{l} 
n \\
\multirow{3}{3}{} \\
$\frac{0}{0}$ \\
$\frac{0}{3}$ \\
3
\end{tabular} & $\begin{array}{l}3 \\
\frac{3}{0} \\
\frac{0}{3} \\
\stackrel{3}{0} \\
\frac{0}{3}\end{array}$ \\
\hline 1 & $2 * 60$ & 0 & 0 & 0 & 0 & 2 & 0 & Yes $^{2}$ \\
\hline 2 & $2 * 60$ & 0 & 0 & 0 & 0 & 1 & 0 & No \\
\hline 3 & none & NA & NA & NA & NA & NA & NA & NA \\
\hline 4 & $2 * 60$ & 0 & 0 & 0 & 0 & 0 & 0 & No \\
\hline 5 & 120 & 0 & 0 & 0 & 0 & 2 & 0 & Yes $^{2}$ \\
\hline 6 & 120 & 0 & 0 & 0 & 0 & 2 & 0 & Yes $^{2}$ \\
\hline 7 & 120 & 0 & 0 & 0 & 0 & 1 & 0 & No \\
\hline
\end{tabular}

Toxicity scored before study entry (baseline) and at days 7 and 14 after cetuximab. NA: not assessed because of premature exclusion from the study. ${ }^{1}$ Every change in vital signs or medication compared to the baseline values was scored, with " $O$ " indicating no change. ${ }^{2}$ Doxycycline 100 $\mathrm{mg}$, Hydroxyzine $25 \mathrm{mg}$, Metronidazolcream $10 \mathrm{mg} / \mathrm{g}$

\section{Image analysis}

Images of ${ }^{89} \mathrm{Zr}$-cetuximab uptake in the tumour of 2 patients are presented in Figure 2. For these patients, an ${ }^{18} \mathrm{~F}$-FDG-PET scan was performed within 1 week after the ${ }^{89} \mathrm{Zr}$ cetuximab scan. A heterogeneous uptake of ${ }^{89} \mathrm{Zr}$-cetuximab was observed throughout the tumourand the rest of the body. In patient 1 , a remarkable mismatch was observed between FDG- and ${ }^{89} \mathrm{Zr}$-cetuximab uptake (Figure 3 ). For the patients in dose step 1, the average maximum TBR at day 4, 5 and 6 after injection was 0.89 (range: $0.83-0.94$ ), 0.98 (range: 0.88-1.05) and 1.09 (range: 0.76-1.60), respectively. As the most optimal TBR was found at day 6 after injection, imaging in dose step 2 was performed at days 5, 6 and 7. For patients in dose step 2, the average maximum TBR at day 5, 6 and 7 after injection was 0.88 (range: 0.57-1.05), 0.86 (range: 0.54-1.02) and 0.91 (range: 0.42-1.20). Three patients had a maximum TBR $\geq 1$ at any of the imaging time points: patient 4 had a maximal TBR of 1.60, patient 6 had a maximal TBR of 1.20 and the maximal TBR of patient 7 was 1.11. 

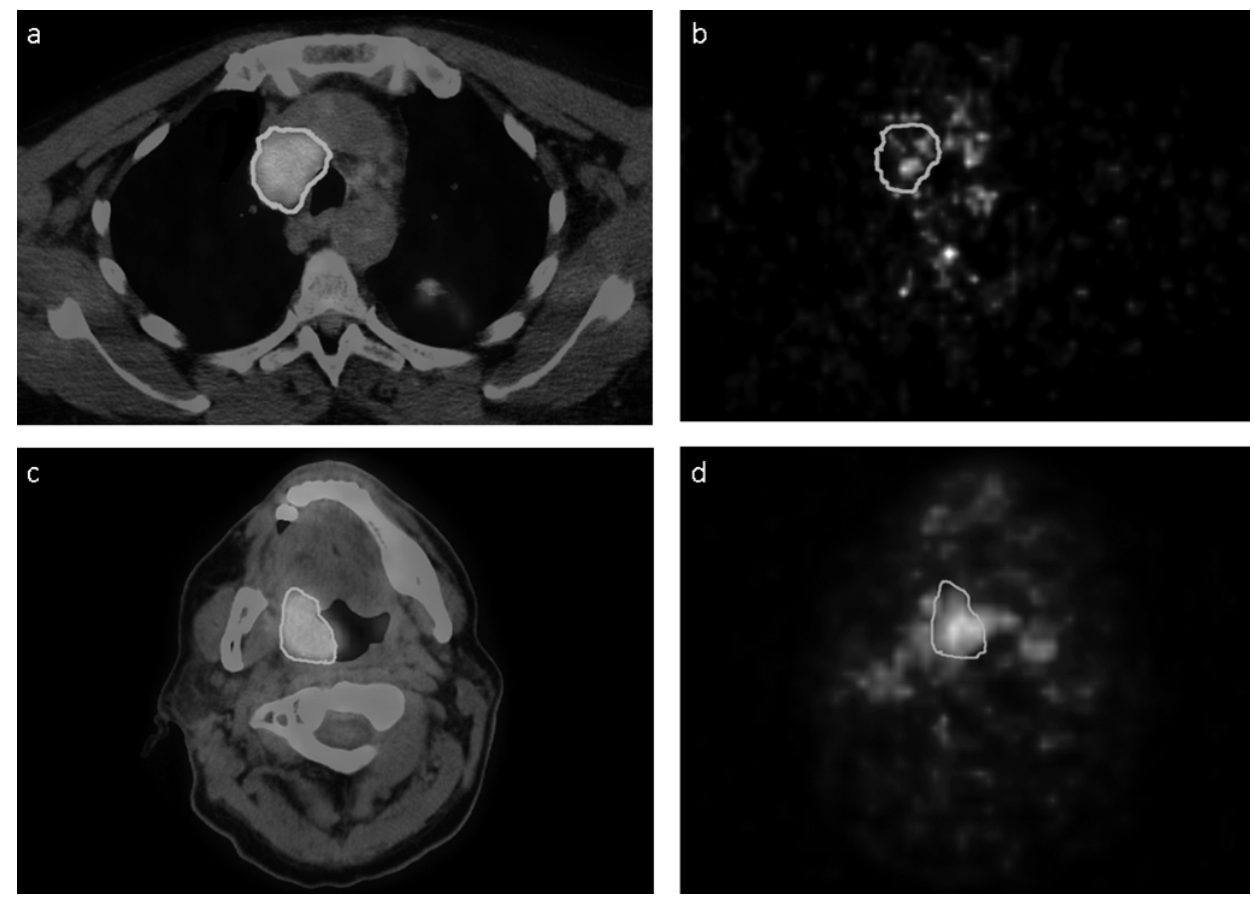

Figure 2.

${ }^{18}$ FDG (left) and ${ }^{89}$ Zr-cetuximab (right) images of two representative patients:

- Patient 6: a) FDG-PET-CT, b) ${ }^{89} \mathrm{Zr}$-cetuximab-PET with TBR=1.20

- Patient 7: c) FDG-PET-CT, d) ${ }^{89}$ Zr-cetuximab-PET with TBR=1.11

${ }^{89} \mathrm{Zr}$-cetuximab images at day 7 after injection are shown. ${ }^{18}$ FDG images were acquired at day 14 after ${ }^{89} \mathrm{Zr}$-cetuximab administration. Note, the heterogeneous uptake of ${ }^{89} \mathrm{Zr}$-cetuximab in the tumours.
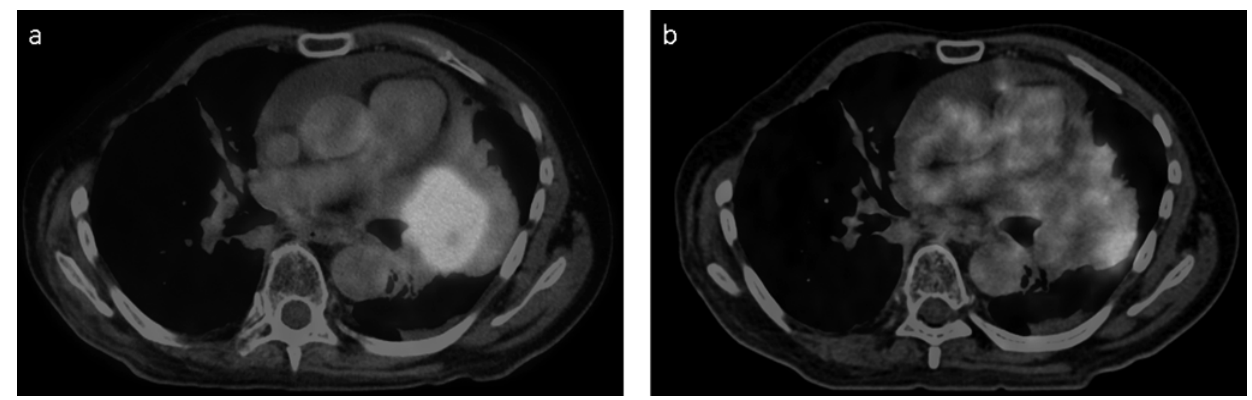

Figure 3.

Correlation between ${ }^{89} \mathrm{Zr}$-cetuximab and ${ }^{18} \mathrm{FDG}$ uptake in patient 1

a) FDG-PET-CT b) ${ }^{89}$ Zr-cetuximab-PET-CT (day 6 after injection) 


\section{DISCUSSION}

Although Zirconium-89 labelled with other monoclonal antibodies has been evaluated in patients before without additional toxicity ${ }^{20}$, the current phase I trial is the first in human study evaluating ${ }^{89} \mathrm{Zr}$ labelled with cetuximab. No additional toxicity was associated with the ${ }^{89} \mathrm{Zr}$ administration. Acne form eruption was observed in $83 \%$ of patients, a rate comparable to that found with the therapeutic administration of cetuxi$\operatorname{mab}^{8,15,21}$. One patient developed malignant hypertension during the administration of unlabelled cetuximab. Although rare, this side-effect has been described previously ${ }^{22}$.

Many preclinical data show additive anti-tumour effects for cetuximab with radiotherapy and chemoradiotherapy in experimental models ${ }^{9,23,24}$. The mechanisms underlying clinical response or resistance to treatment against EGFR with cetuximab are unknown. Until now, the only factors found to be associated with tumour-response to cetuximab are wild-type K-RAS and an increased EGFR gene copy number. EGFR status was not associated with significant differences in response rates or survival. Also, time-frames of EGFR-expression during the course of (chemo)-radiation are largely unknown. EGFR tyrosine kinase activity can be initiated by a single dose of radiation in experimental tumour models ${ }^{25}$. Repeated doses of daily radiation, similar to standard clinical therapy, stimulate increased EGFR expression in experimental models ${ }^{26}$. Visualization and quantification of cetuximab uptake in the tumour with PET before and during (chemo-) radiotherapy would have major benefit in the appropriate selection of patients for cetuximab treatment ${ }^{13}$.

The maximal TBR of ${ }^{89} \mathrm{Zr}$-cetuximab uptake in the patients included in this phase I study was 1.6. Correlation of the ${ }^{89} \mathrm{Zr}$-cetuximab and FDG uptake showed a remarkable mismatch between the two tracers in one patient. Although a large divergence was observed between patients in the time curves of the TBR, overall, the most optimal TBR was observed at the latest time point of imaging, being day 6 for dose step 1 and day 7 for dose step 2. Therefore, we would suggest the time point of 6 to 7 days after therapy to perform Zr-cetuximab imaging.

Several possibilities may explain the low TBRs observed in this study. First, the results may represent true negative findings in this selective patient group. Biopsies of the primary tumours of the patients enrolled in this study did not show overexpression of EGFR or a mutation in the EGFR gene. All patients, however, had undergone extensive treatment with chemotherapy or radiotherapy, of which the long term effect on EGFR expression is largely unknown. Five of the 6 included patients had NSCLC. Although 40$80 \%$ of NSCLC tumours show EGFR expression ${ }^{1}$, response rates to cetuximab monotherapy in pre-treated patients with EGFR expressing primary tumours of only $5 \%$ are reported ${ }^{21}$. Second, the loading dose administered in this study $\left(400 \mathrm{mg} / \mathrm{m}^{2}\right)$, which is 
the standard in therapeutic regimens might be either too low or too high to enable adequate tumour visualization. The rationale of the loading dose of cetuximab is that cetuximab first binds irreversibly to EGFR expressing liver cells, and that tumour cells are only targeted after the liver has been saturated ${ }^{27}$. This rationale is supported by animal studies as well as by the positive relation between skin toxicity and tumour response to cetuximab, implying that skin saturation only occurs after the liver has been saturated ${ }^{28-30}$. On the other hand, it cannot be excluded that the unlabelled cetuximab is already sufficient to saturate the EGF receptors on the tumour cells. Future studies should address these critical questions. Finally, it is possible that cetuximab might not reach its target receptor at tumour cells due to limited blood supply and interstitial pressure of the tumour ${ }^{31}$. Hereby, a disparity would result between the in vivo EGFR expression and the uptake of cetuximab, a finding that has been confirmed in our preclinical study with ${ }^{89} \mathrm{Zr}$-cetuximab ${ }^{14}$.

Future studies are planned addressing the role of each of these contributing factors. Patients will be studied at primary diagnosis before the start of any antitumour therapy, to allow correlation of EGFR expression at histology with imaging results. As radiotherapy induces upregulation of EGFR expression, visualization of ${ }^{89} \mathrm{Zr}$-cetuximab uptake before and after a single fraction of RT will be evaluated. ${ }^{89} \mathrm{Zr}$-cetuximab administration with different loading doses of unlabelled cetuximab will be evaluated to address its influence on tumour visualization. Finally, kinetic imaging enables the measurement of tumour perfusion, and this should thus be incorporated in future clinical trials.

Concluding, the administration of ${ }^{89} \mathrm{Zr}$ labelled cetuximab to image EGFR expression in vivo is not associated with any additional toxicity. Based on the tumour to background ratios, a time delay of 6-7 days after injection is advised for further research. 


\section{REFERENCES}

1. Rowinsky EK. The erbB family: targets for therapeutic development against cancer and therapeutic strategies using monoclonal antibodies and tyrosine kinase inhibitors. Annu Rev Med 2004;55:433-57.

2. Herbst RS. Review of epidermal growth factor receptor biology.Int J Radiat Oncol Biol Phys 2004;59:21-6.

3. Akimoto T, Hunter NR, Buchmiller L, Mason K, Ang KK, Milas L. Inverse relationship between epidermal growth factor receptor expression and radiocurability of murine carcinomas. Clin Cancer Res 1999;5:2884-90.

4. Ang KK, Berkey BA, Tu X, et al. Impact of epidermal growth factor receptor expression on survival and pattern of relapse in patients with advanced head and neck carcinoma. Cancer Res 2002;62:7350-6.

5. Baumann M, Krause M, Zips D, et al. Selective inhibition of the epidermal growth factor receptor tyrosine kinase by BIBX1382BS and the improvement of growth delay, but not local control, after fractionated irradiation in human FaDu squamous cell carcinoma in the nude mouse. Int J Radiat Biol 2003;79:547-59.

6. Gibson MK, Abraham SC, Wu TT, et al. Epidermal growth factor receptor, p53 mutation, and pathological response predict survival in patients with locally advanced esophageal cancer treated with preoperative chemoradiotherapy. Clin Cancer Res 2003;9:6461-8.

7. Giralt J, de las Heras M, Cerezo L, et al. The expression of epidermal growth factor receptor results in a worse prognosis for patients with rectal cancer treated with preoperative radiotherapy: a multicenter, retrospective analysis. Radiother Oncol 2005;74:101-8.

8. Bonner JA, Harari PM, Giralt J, et al. Radiotherapy plus cetuximab for squamous-cell carcinoma of the head and neck.N Engl J Med 2006;354:567-78.

9. Raben D, Helfrich B, Chan DC, et al. The effects of cetuximab alone and in combination with radiation and/or chemotherapy in lung cancer.Clin Cancer Res 2005;11:795-805.

10. Rasbridge SA, Gillett CE, Seymour AM, et al. The effects of chemotherapy on morphology, cellular proliferation, apoptosis and oncoprotein expression in primary breast carcinoma. $\mathrm{Br}$ J Cancer 1994;70:335-41.

11. Contessa JN, Reardon DB, Todd D, et al. The inducible expression of dominant-negative epidermal growth factor receptor-CD533 results in radiosensitization of human mammary carcinoma cells. Clin Cancer Res 1999;5:405-11.

12. Schmidt-Ullrich RK, Contessa JN, Dent $P$, et al. Molecular mechanisms of radiation-induced accelerated repopulation. Radiat Oncol Investig 1999;7:321-30.

13. van Dongen GA, Visser GW, Lub-de Hooge MN, de Vries EG, Perk LR. Immuno-PET: a navigator in monoclonal antibody development and applications. Oncologist 2007;12:1379-89.

14. Aerts HJ, Dubois L, Perk L, et al. Disparity between in vivo EGFR expression and 897rlabeled cetuximab uptake assessed with PET. J Nucl Med 2009;50:123-31.

15. Galimont-Collen AF, Vos LE, Lavrijsen AP, Ouwerkerk J, Gelderblom H. Classification and management of skin, hair, nail and mucosal side-effects of epidermal growth factor receptor (EGFR) inhibitors. Eur J Cancer 2007;43:845-51.

16. Verel I, Visser GW, Boellaard R, Stigter-van Walsum M, Snow GB, van Dongen GA. 89Zr immuno-PET: comprehensive procedures for the production of $89 \mathrm{Zr}$-labeled monoclonal antibodies. J Nucl Med 2003;44:1271-81. 
17. Perk LR, Visser GW, Vosjan MJ, et al. (89)Zr as a PET surrogate radioisotope for scouting biodistribution of the therapeutic radiometals (90)Y and (177)Lu in tumor-bearing nude mice after coupling to the internalizing antibody cetuximab. J Nucl Med 2005;46:1898-906.

18. Daisne JF, Sibomana M, Bol A, Doumont T, Lonneux M, Gregoire V. Tri-dimensional automatic segmentation of PET volumes based on measured source-to-background ratios: influence of reconstruction algorithms. Radiother Oncol 2003;69:247-50.

19. Ollers $M$, Bosmans $G$, van Baardwijk $A$, et al. The integration of PET-CT scans from different hospitals into radiotherapy treatment planning. Radiother Oncol 2008;87:142-6.

20. Borjesson PK, Jauw YW, Boellaard R, et al. Performance of immuno-positron emission tomography with zirconium-89-labeled chimeric monoclonal antibody U36 in the detection of lymph node metastases in head and neck cancer patients. Clin Cancer Res 2006;12:2133-40.

21. Hanna N, Lilenbaum R, Ansari R, et al. Phase II trial of cetuximab in patients with previously treated non-small-cell lung cancer. J Clin Oncol 2006;24:5253-8.

22. European Medicines Agency.

23. Huang SM, Bock JM, Harari PM. Epidermal growth factor receptor blockade with C225 modulates proliferation, apoptosis, and radiosensitivity in squamous cell carcinomas of the head and neck. Cancer Res 1999;59:1935-40.

24. Milas L, Mason K, Hunter N, et al. In vivo enhancement of tumor radioresponse by C225 antiepidermal growth factor receptor antibody.Clin Cancer Res 2000;6:701-8.

25. Schmidt-Ullrich RK, Valerie K, Fogleman PB, Walters J. Radiation-induced autophosphorylation of epidermal growth factor receptor in human malignant mammary and squamous epithelial cells. Radiat Res 1996;145:81-5.

26. Schmidt-Ullrich RK, Valerie KC, Chan W, McWilliams D. Altered expression of epidermal growth factor receptor and estrogen receptor in MCF-7 cells after single and repeated radiation exposures. Int J Radiat Oncol Biol Phys 1994;29:813-9.

27. Divgi $C R$, Welt $S$, Kris $M$, et al. Phase I and imaging trial of indium 111-labeled antiepidermal growth factor receptor monoclonal antibody 225 in patients with squamous cell lung carcinoma. J Natl Cancer Inst 1991;83:97-104.

28. Burtness B, Goldwasser MA, Flood W, Mattar B, Forastiere AA. Phase III randomized trial of cisplatin plus placebo compared with cisplatin plus cetuximab in metastatic/recurrent head and neck cancer: an Eastern Cooperative Oncology Group study. J Clin Oncol 2005;23:864654.

29. Cunningham D, Humblet $Y$, Siena $S$, et al. Cetuximab monotherapy and cetuximab plus irinotecan in irinotecan-refractory metastatic colorectal cancer. N Engl J Med 2004;351:337-45.

30. Tolmachev $\mathrm{V}$, Rosik $\mathrm{D}$, Wallberg $\mathrm{H}$, et al. Imaging of EGFR expression in murine xenografts using site-specifically labelled anti-EGFR 111In-DOTA-Z EGFR:2377 Affibody molecule: aspect of the injected tracer amount. Eur J Nucl Med Mol Imaging;37:613-22.

31. de Bree R, Kuik DJ, Quak JJ, et al. The impact of tumour volume and other characteristics on uptake of radiolabelled monoclonal antibodies in tumour tissue of head and neck cancer patients. Eur J Nucl Med 1998;25:1562-5. 


\section{CHAPTER}

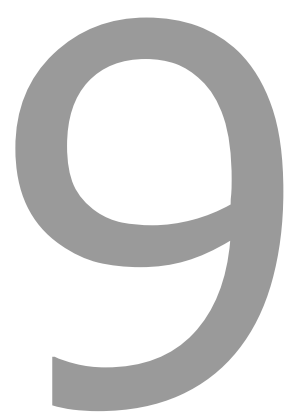

General discussion and future perspectives 


\section{GENERAL DISCUSSION}

Due to disappointing outcomes in the treatment of cancer, poor local tumour control, and the heterogeneous response of patients with the same diagnosis to a specific treatment, it has become increasingly clear that tailored treatments are potentially beneficial. This problem is addressed by "personalized medicine" were the treatment is individualized towards the specific characteristics of a patient, instead of the currently common "one size fits all" treatment modalities. In personalized medicine there is a growing interest to individualize treatment by the combination of systemic therapies and dose painting of radiation, as high precision radiotherapy is capable of directing a therapeutic effect in the three dimensions of space towards areas were other treatments are failing. It is expected that the implementation of these techniques in clinical settings will lead to improved local control of the tumour without an increase of the side effects, and consequently improved survival and quality of life. The concept of dose painting takes advantage of intratumour differences in the sensitivity for radiotherapy, chemotherapy, targeted agents, and their combinations, as a tumour can be composed of large spacial differences in biologic characteristics related with treatment resistance. A precondition for dose painting is that the areas where it is likely that treatment will fail can be identified, i.e. areas with biologic resistance to treatment (investigated in part 1 of this thesis) and areas with limited drug uptake (investigated in part 2). Here, molecular imaging modalities able to quantify these intra tumour areas with differences in biologic characteristics and drug uptake before and even during treatment make this a feasible strategy. Currently, many investigators remained sceptic about the implementation of dose painting in clinical settings, mainly because the scientific basis for these studies was missing.

However, the work presented in this thesis showed evidence of both the pretreatment identification of intra tumour areas with a high probability of treatment failure, as well as the non-invasive visualisation of heterogeneous drug uptake in tumours. Based on the results of this thesis and previous knowledge of FDG, we postulate that there is a scientific basis to test dose painting in clinic settings, which can lead to new paradigms in radiotherapy combined with systemic treatment.

\section{Intra-tumour heterogeneity}

A prerequisite for intra-tumour dose painting is that a tumour is heterogeneous in regions of treatment sensitivity. Indeed, several studies have shown that there are large intra-tumour spatial variations in biologic characteristics, like proliferation, hypoxia, cell density, perfusion, vasculature, gene expression, but also drug uptake. Therefore, it is likely that there are large spatial differences in the probability to eradicate distinguishable tumour sub-volumes by radiotherapy, chemotherapy, targeted agents, and 
or their combinations. The results in this thesis confirmed these findings and showed a heterogeneous pattern of the glucose analogue ${ }^{18} \mathrm{~F}$-deoxyglucose (FDG) for positron emission tomography (PET) imaging before (Chapter 2-4) and during treatment (Chapter 5), where the high FDG uptake areas was often not in the centre of the tumour. Also, a heterogeneous pattern was observed for Cetuximab uptake in pre-clinical settings. Ex vivo immunohistochemistryanalysis showed a very heterogeneous uptake of in vivo Cetuximab, but also a very heterogeneous EGFR expression throughout the tumour (Chapter 6). Also, in in vivo PET images with ${ }^{89} \mathrm{Zr}$-Cetuximab large intra-tumour heterogeneity was observed in mice (Chapter 7) and in patients (Chapter 8).

\section{The numbers of cells are critical for resistance}

On might wonder if the volumes of treatment-resistance are sufficiently large to be identified with imaging and for current dose painting techniques. Several biologic characteristics have been associated with treatment-resistance, like hypoxia, stem cells, proliferation, metabolic activity, and cell density. In the case of hypoxia for instance, a larger hypoxic volume results in a worse survival ${ }^{1-4}$. This and other examples such as the metabolic volume based on FDG underline that factors related to resistance are continuous and not dichotomous parameters ${ }^{5}$. Therefore, it can be agrued that overt treatment-resistance will mostly occur when sub-volumes within the tumour reach a critical volume, which may be detectable by current imaging modalities. Therefore, we argue that the numbers of cells are critical for resistance, i.e. for treatment resistance there should be a critical volume that is measurable by imaging. Indeed, hypoxic volumes from approximately $2-3 \mathrm{~cm}^{3}$ or more are problematic to control, not some sparse hypoxic cells spread out throughout of the tumour ${ }^{1}$. This is in agreement with direct $\mathrm{pO}_{2}$ measurements with Eppendorf electrodes, which are not capable to measure microscopic hypoxia, but still can distinguish prognostic subgroups ${ }^{1,6,7}$. As the minimal clinical relevant resistant volume is probably $1-2 \mathrm{~cm}^{3}$ and the technical possibility for radiotherapy to deliver doses is restricted to the same volume, even with these limitations, relevant radiation dose re-distribution may be feasible.

\section{FDG for the identification of radioresistance}

Probably the most investigated methodology for visualizing intra tumour radioresistance are hypoxia tracers, as hypoxia is known to be strongly correlated with radiation sensitivity ${ }^{8,9}$. However, the currently available hypoxia tracers show limitations for its applicability, e.g. low test re-test performance and low tumour to reference activity ratios ${ }^{10-12}$. Also, as hypoxia is only one of several characteristics linked with radioresistance, it is not well known how hypoxia tracers directly relate to radioresistance. Furthermore, it is unknown if intra tumour areas with high tracer uptake have the highest probability to relapse. Due to these reasons, we believe more evi- 
dence is desirable to use hypoxia tracers for dose painting in the clinic. Another tracer suggested for dose painting is FDG. The underlying biology for FDG uptake is complex because it measures the uptake of glucose in cells by ATP independent glucose transporters. This makes FDG uptake specific to the metabolic pathway, although this pathway is known to be influenced by numerous other biological processes related to radio-resistance, like proliferation ${ }^{13}$, cell density ${ }^{14}$, and hypoxia ${ }^{15,16}$. Also, the maximal uptake of FDG in the tumour is prognostic for survival of patients with NSCLC, both treated with surgery or radiotherapy ${ }^{16-20}$. Furthermore, FDG is widely available at almost all oncology centres in developed countries. Therefore, we also consider FDG as a rational choice for dose painting studies. However, it should be stated that there are also limitations with FDG, such as aspecific uptake in inflammatory tissue. Also, as metabolism is only one tumour characteristic of importance, it is expected that multiple tracers will yield better estimations of treatment sensitivity.

\section{Identification of residual metabolic active areas}

We hypothesized that the location of the residual metabolic-areas following treatment are probably the areas that are the most radio-resistant and can be identified before treatment starts using a single FDG-PET-CT scan. Therefore, we investigated, using an overlap fraction (OF) calculation, if the areas of residual metabolic activity after treatment had a large overlap with the pre-treatment high FDG-uptake within the primary tumour. An analysis was performed in a dataset of patients treated at MAASTRO consisting about high-dose radiotherapy, mostly preceded by chemotherapy (Chapter 2). The results showed that the pre-treatment high FDG-uptake areas largely corresponded with the metabolic active areas after treatment. Also, the residual areas were located almost completely within the pre-treatment GTV volume, indicating no geographic miss of radiotherapy. Validations of these results were performed in an independent dataset from the Princess Margaret Hospital in Toronto (Chapter 3) and in an independent prospectively gathered dataset from the Radboud University Medical Centre in Nijmegen (Chapter 4). The Nijmegen dataset yielded similar results with the same thresholds as the MAASTRO dataset. Also, the Toronto dataset yielded similar results, however with the pre-treatment $40 \% \mathrm{SUV}_{\max }$ high uptake threshold. That the $40 \%$ SUV threshold was used instead of the $50 \%$ threshold was due to differences of the absolute values and volumes of the thresholds between the Toronto dataset and the Dutch datasets. This may be due to the PET protocol that differed substantially, as MAASTRO and Nijmegen used the standardization NEDPAS protocol ${ }^{21,22}$ and Toronto did not. This underscores the necessity for thorough standardization before multicentre studies can be launched. An important limitation of the used analysis, is that for the registration between the pre- and post scan a rigid registration was used, not incorporating deformable tissue changes, possible induced by therapy. This can be improved by using non-rigid registration methods, incorporating these deformations. 
However, these techniques are difficult to validate and the reproducibility between institutes is limited. Therefore, in every dataset patients were excluded from analysis that showed large deformations of the normal tissues near the tumour.

Although our surrogate endpoint, persistent FDG uptake in the tumour, should not be regarded as equivalent to tumour persistence, we argued that residual metabolic active areas indicate areas of treatment resistance. In agreement with literature ${ }^{5}$, patients with persistent FDG uptake showed a significantly worse survival than those without, underlining its clinical validity (Chapter 2). Based on these results, we conclude that residual metabolic active areas within the tumour are probably more radioresistant. Another study from our group investigated the relationship between FDG uptake in a tumour subvolume (what we call a voxel) and the probability that voxels contained no residual metabolic disease ${ }^{23}$. We referred to this probability as voxel control probability (VCP). Apart from FDG-uptake, the effect of VCP on radiation dose, gross tumour volume, the $S_{U} V_{\max }$ and the administration of chemotherapy before irradiation was investigated. It was shown that VCP decreased with increasing FDG uptake $(p<0.001)$ and increasing GTV $(p<0.001)$. No statistically significant dose effect relationship was found. However, according to Martel et al. a dose of $84 \mathrm{~Gy}$ is needed for a $50 \%$ tumour control ${ }^{24}$. The patients in this dataset received doses ranging from 50.4 Gy (given in 28 twice-daily fractions of $1.8 \mathrm{~Gy}$ ) to $79.2 \mathrm{~Gy}$ (44 twice-daily fractions). Therefore it is likely that we are looking at the shallow part of the slope of the TCP curve in our patients, making it difficult to detect a relationship between dose and VCP. Although no dose effect relationship was found, these findings confirm that high FDG uptake voxels are more likely to contain residual metabolic disease after therapy and that more dose is needed to control them.

\section{Pre-clinical and clinical in vivo imaging of Cetuximab}

The visualisation of a drug like Cetuximab is of importance, as a lot is unknown about patient-specific tumour uptake and overall pharmacokinetics of Cetuximab ${ }^{25-27}$. Moreover, only a small sub-population of patients benefits from Cetuximab treatment, were often discrepancies between Cetuximab uptake and EGFR expression levels have been reported ${ }^{28-30}$. For the visualisation, we choose the long-lived positron emitter Zirconium-89 $\left({ }^{89} \mathrm{Zr}\right)$ for the labelling with Cetuximab. ${ }^{89} \mathrm{Zr}$ has ideal characteristics for in vivo imaging, e.g. the long half life (78 hours) that enables imaging up to a week after injection, sufficient for the biologic half life of Cetuximab in the blood pool, but also the very high labelling efficiency of ${ }^{89} \mathrm{Zr}$-Cetuximab (immunoreactive fraction $>95 \%$ ). Also, ${ }^{89} \mathrm{Zr}$-Cetuximab can be produced under good manufacturing procedure (GMP) settings, making it an ideal candidate for a fast translation to the clinic ${ }^{31}$. In vivo PET images in mice showed successful imaging that revealed a clear uptake of ${ }^{89} \mathrm{Zr}$-Cetuximab in 
EGFR positive tumours with high quality images (tumour-to-blood ratios from 3-5) (Chapter 7).

\section{Disparity between EGFR expression and Cetuximab uptake}

To assess the binding of Cetuximab in vitro, an image probe using Cetuximab labelled with the fluorescent marker Oregon-Green 488 was developed (Chapter 6). This probe showed that in vitro the binding of Cetuximab was strongly related with EGFR expression levels. However, ex vivo immunohistochemistryanalysis showed a very heterogeneous uptake of in vivo Cetuximab, but also a very heterogeneous EGFR expression throughout the tumour. Besides being heterogeneous, a disparity was reported were areas with low EGFR expression had a high Cetuximab uptake, and vice versa. In a preclinical study in mice, again a large disparity was found between ${ }^{89} \mathrm{Zr}$-Cetuximab uptake and in vivo EGFR expression levels (Chapter 7). The uptake in a high EGFR expressing cell line was two times lower than in intermediate EGFR expressing cell lines. These results were confirmed with ex vivo $\mathrm{Y}$-counting biodistribution studies. A phase I imaging trial using ${ }^{89} \mathrm{Zr}$-Cetuximab in cancer patients showed no associated toxicity and selective targeting of ${ }^{89} \mathrm{Zr}$-Cetuximab in the tumour, although with very low tumour-toblood ratios (Chapter 8 ). This was expected as Cetuximab is only efficient in a small number of patients, and only six patients successfully finished the study, and all of these patients where EGFR negative.

Importantly, the disparity described in this thesis may help to explain the poor correlation reported between Cetuximab efficacy and EGFR expression, as the results reveal additional mechanisms that can explain why EGFR expression levels alone may not be sufficient to explain therapy effects of Cetuximab in the clinic. A possible explanation for this disparity can be a poor drug delivery of the relatively large antibody ( $\pm 152 \mathrm{kDa}$ ) into the tumour tissue due to inadequate vasculature, differences in capillary permeability perfusion, and differences in necrosis. Also, the internalization rate of the Cetuximab-EGFR complex may be different per cell type. However, the biologic reason for this disparity needs further investigation.

\section{Treatment efficacy of drugs}

We hypothesize that for effective treatment of a drug like Cetuximab there are two prerequisites:

1 the drug has to be able to reach all areas within the tumour.

2 the cells have to be sensitive for the drug.

Therefore, if Cetuximab is not able to reach the all tumour regions, no therapeutic effect can be expected in these regions. Here, Cetuximab imaging can be used for exclu- 
sion of this expensive treatment of patients with no (or limited) uptake. However, if Cetuximab is able to reach a tumour region, this does not guarantee there will be a therapeutic effect given a possible Cetuximab resistance of the cells. This resistance and sensitivity for Cetuximab treatment may be associated with EGFR expression levels, however, can also be more complex due to overall redundancy in the EGFR pathway. It is known that cancer cells can bypass the dependency of EGFR for cell growth and survival by altering EGFR levels through promoting EGFR ubiquitination and degradation, resulting in Cetuximab resistance ${ }^{32}$. Overall, the relationship between EGFR expression levels, Cetuximab binding and, ultimately, clinical outcome is complex. In vitro a strong relationship between Cetuximab binding and EGFR expression levels was found (Chapter 6). Also, high EGFR expression is correlated with poor clinical outcome 27. However, no correlation is found between the efficacy of Cetuximab treatment and EGFR expression ${ }^{28-30}$. Therefore, in future clinical trials ${ }^{89} \mathrm{Zr}$-Cetuximab may be used to: I) omit non-beneficial patients with no or limited uptake, and II) optimize Cetuximab dosage in patients with tumour targeting, possibly complemented with dose painting of radiation.

\section{Adaptation of treatment based on imaging}

In general, the results of this thesis show that non-invasive imaging of biologic characteristics like metabolism can be used to identify the regions of radioresistance. Therefore, a heterogeneous dose distribution, matching the profile of intra tumour differences of radio-curability, seems to be a rational choice. Imaging of drugs can, besides selecting beneficial subpopulation for specific therapies and optimizing dosage, be used for identifying regions with limited drug uptake that can be compensated with dose painting.For the introduction of dose painting in the clinic intensity modulation radiotherapy (IMRT) has often been suggested. IMRT is an advanced technique that uses computer controlled linear accelerators to deliver a precise three dimensional radiation dose distribution to the tumour by controlling the radiation beam in multiple small volumes. Therefore, the total dose to the tumour can be maintained while reducing the dose to the surrounding normal tissues, resulting in less toxicity. Currently, IMRT is often used in clinical settings for patients with difficult treatment plans, e.g. due to tumours that are closely to other organs. For the utility of dose painting using Image-Guided Radiotherapy, IMRT can be used to deliver a higher dose to intra tumour sub-volumes that are more resistant, while reducing the dose towards the more sensitive tumour sub-volumes. However, this can result in treatment plans with large differences of dose between adjacent voxels. These are considered unrealistic because of movement of tumour and organs during the treatment, shrinkage of the tumour due to the treatment, day to day differences in patient positioning, and the limited accuracy of imaging and voxel-wise dose delivery techniques. Therefore, larger tumour subvolumes are more realistic for dose painting as the dose gradient per voxel can be less 
steep. Therefore, with current Image-Guided Radiotherapy techniques, adaptive therapy has now become challenging, but realistic.

\section{FUTURE PERSPECTIVES}

The results of this thesis form a solid basis for future research in both clinical and preclinical settings. The results of part 1 show 'evidence' to test personalized medicine using dose painting in clinical settings, by individualizing treatment based on the high FDG uptake areas before treatment. Based on these results, a phase II dose painting trial is currently ongoing at MAASTRO Clinic together with the Dutch Cancer Institute (NKI/AvL) in Amsterdam. In this trial patients with inoperable stage II or III NSCLC are randomized to receive an integrated boost to the primary tumour as a whole ( $\operatorname{arm} A)$ or with an integrated boost to the $50 \%$ SUV $_{\max }$ area of the primary tumour (arm B). After one year local tumour control is assessed and overall survival is monitored of all patients. It is expected that these efforts will increase local tumour control and thereby improve survival and quality of life. If successful, this trial can set an example of dose painting trials. In general, the methodology presented in part 1 of this thesis can be used in future studies to identify the intra tumour location where a treatment modality fails. This can be used in evaluation of radiotherapy in combination with systemic therapies, as well as targeted therapies. Also other tracers could be used, like hypoxia tracers. However, first further investigations have to be performed on standardisation and assessment of the location of relapse. Therefore, for the time being FDG represents a rational choice to be used in this context.

Besides imaging the biologic characteristics of a tumour, non-invasive imaging of drugs can play an increasing role in the future for treatment individualization. Imaging of drug delivery has the potential to identify patients that show no uptake of the drug in the tumour and thus are expected not to benefit from it, and to optimize the dosage in patients with selective tumour targeting. Our results show that Cetuximab uptake is not only dependent on EGFR expression levels, but also on additional mechanisms. A possible explanation for this disparity can be a poor drug delivery into the tumour tissue due to inadequate vasculature, differences in capillary permeability perfusion, and differences in necrosis. In a pre-clinical setting it is possible to assess EGFR expression and tumour perfusion in a panel of tumours, and relate this to Cetuximab uptake. Also, it is possible to change the vasculature of xenographs using other drugs and assess if the uptake of Cetuximab is altered. Furthermore, the relationship between Cetuximab dose, ${ }^{89} \mathrm{Zr}$-Cetuximab uptake, and treatment efficacy can be investigated. In clinical settings, it is interesting to assess if tumour uptake of ${ }^{89} \mathrm{Zr}$-Cetuximab is related with Cetuximab dose and treatment efficacy as we believe that the response rate of 
Cetuximab treatment is largely determined by the accessibility of the antibody in the tumour tissue. Possibly, in a phase II trial patient specific distribution and sensitivity of Cetuximab can be assessed using ${ }^{89} \mathrm{Zr}$-Cetuximab, to select and monitor patients that could benefit from Cetuximab treatment. Using multiple image time points during treatment the uptake can be monitored before and during treatment and the outcome can be assessed using differences in metabolic response rate after treatment between patients with a high uptake vs. low uptake on the ${ }^{89} \mathrm{Zr}$-Cetuximab scans.

Antibody imaging addresses a frequent debate that is taking place within the international scientific community, about the affordability of new expensive drugs (e.g. Cetuximab, Trastuzumab, Bevacizumab, Retuximab), because patient selection is currently very difficult. Consequently, the scientific and medical community has shown a lot of interest in the results of studies like this. Both pre-clinical and clinical investigations presented in this thesis show a methodology to non-invasively image the biodistribution of therapeutics, allowing faster and especially more effective product development and clinical trials, possibly leading to better and cheaper medicines. We believe that the development of new therapeutic agents should be accompanied with visualisation of drug delivery and the tumour characteristics which it targets. At the end, this will allow better selection of patients whom can benefit from therapeutic drugs in combination with conventional treatment modalities, resulting in individualized patient treatment. Since the total revenue of drugs like Cetuximab is very high, even a method for excluding a relatively small fraction of all patients can already save a considerable amount of money, which would have otherwise been spent on unnecessary administrations with possible toxicities. Consequently, this can result in a loss of market share of existing drugs and can have large financial consequences. However, the benefit of more effective drug development will benefit the pharmaceutical industry as well.

In the future, both imaging of biologic characteristics and imaging of drug uptake can be combined to tailor the treatment to a specific patient. Both the biologic treatment resistant areas and the areas with limited drug uptake should receive an increased radiation dose, while decreasing the dose to sensitive regions with drug uptake. The exact dose that these resistant and low drug uptake areas must receive, should be tested in carefully designed clinical trials. In general, these studies demonstrate how advances in molecular imaging, even using conventional tracers as FDG, directly facilitate individualized treatment in Radiation Oncology. 


\section{REFERENCES}

1. Nordsmark M, Bentzen SM, Rudat V, et al., Prognostic value of tumor oxygenation in 397 head and neck tumors after primary radiation therapy. An international multi-center study, Radiother Oncol 77 (1), 18-24 (2005).

2. Fyles $A$, Milosevic $M$, Pintilie $M$, et al., Long-term performance of interstial fluid pressure and hypoxia as prognostic factors in cervix cancer, Radiother Oncol 80 (2), 132-137 (2006).

3. Brizel DM, Sibley GS, Prosnitz LR, et al., Tumor hypoxia adversely affects the prognosis of carcinoma of the head and neck, Int J Radiat Oncol Biol Phys 38 (2), 285-289 (1997).

4. Kaanders JH, Wijffels KI, Marres HA, et al., Pimonidazole binding and tumor vascularity predict for treatment outcome in head and neck cancer, Cancer Res 62 (23), 7066-7074 (2002).

5. Mac Manus MP, Hicks RJ, Matthews JP, et al., Metabolic (FDG-PET) response after radical radiotherapy/chemoradiotherapy for non-small cell lung cancer correlates with patterns of failure, Lung Cancer 49 (1), 95-108 (2005).

6. Nordsmark M, Loncaster J, Aquino-Parsons C, et al., The prognostic value of pimonidazole and tumour $\mathrm{pO} 2$ in human cervix carcinomas after radiation therapy: a prospective international multi-center study, Radiother Oncol 80 (2), 123-131 (2006).

7. Nordsmark M and Overgaard J, A confirmatory prognostic study on oxygenation status and loco-regional control in advanced head and neck squamous cell carcinoma treated by radiation therapy, Radiother Oncol 57 (1), 39-43 (2000).

8. Kim $\mathrm{Y}$, Lin $\mathrm{Q}$, Glazer PM, et al., Hypoxic tumor microenvironment and cancer cell differentiation, Curr Mol Med 9 (4), 425-434 (2009).

9. Thorwarth $D$, Eschmann SM, Paulsen F, et al., Hypoxia dose painting by numbers: a planning study, Int J Radiat Oncol Biol Phys 68 (1), 291-300 (2007).

10. Dubois L, Landuyt W, Cloetens L, et al., [18F]EF3 is not superior to [18F]FMISO for PETbased hypoxia evaluation as measured in a rat rhabdomyosarcoma tumour model, Eur J Nucl Med Mol Imaging 36 (2), 209-218 (2009).

11. Krohn KA, Link JM and Mason RP, Molecular imaging of hypoxia, J Nucl Med 49 Suppl 2, 129S-148S (2008).

12. Bentzen L, Keiding S, Horsman MR, et al., Assessment of hypoxia in experimental mice tumours by [18F]fluoromisonidazole PET and pO2 electrode measurements. Influence of tumour volume and carbogen breathing, Acta Oncol 41 (3), 304-312 (2002).

13. Vesselle $H$, Schmidt RA, Pugsley JM, et al., Lung cancer proliferation correlates with [F18]fluorodeoxyglucose uptake by positron emission tomography, Clin Cancer Res 6 (10), 3837-3844 (2000).

14. Dooms C, van Baardwijk A, Verbeken E, et al., Association between 18F-fluoro-2-deoxy-Dglucose uptake values and tumor vitality: prognostic value of positron emission tomography in early-stage non-small cell lung cancer, J Thorac Oncol 4 (7), 822-828 (2009).

15. Airley R, Loncaster J, Davidson S, et al., Glucose transporter glut-1 expression correlates with tumor hypoxia and predicts metastasis-free survival in advanced carcinoma of the cervix, Clin Cancer Res 7 (4), 928-934 (2001). 
16. van Baardwijk A, Dooms C, van Suylen RJ, et al., The maximum uptake of (18)Fdeoxyglucose on positron emission tomography scan correlates with survival, hypoxia inducible factor-1alpha and GLUT-1 in non-small cell lung cancer, Eur J Cancer 43 (9), 13921398 (2007).

17. Vansteenkiste JF, Stroobants SG, Dupont PJ, et al., Prognostic importance of the standardized uptake value on (18)F-fluoro-2-deoxy-glucose-positron emission tomography scan in non-small-cell lung cancer: An analysis of 125 cases. Leuven Lung Cancer Group, J Clin Oncol 17 (10), 3201-3206 (1999).

18. Eschmann SM, Friedel G, Paulsen F, et al., Is standardised (18)F-FDG uptake value an outcome predictor in patients with stage III non-small cell lung cancer?, Eur J Nucl Med Mol Imaging 33 (3), 263-269 (2006).

19. Borst GR, Belderbos JS, Boellaard R, et al., Standardised FDG uptake: a prognostic factor for inoperable non-small cell lung cancer, Eur J Cancer 41 (11), 1533-1541 (2005).

20. Downey RJ, Akhurst T, Gonen M, et al., Preoperative F-18 fluorodeoxyglucose-positron emission tomography maximal standardized uptake value predicts survival after lung cancer resection, J Clin Oncol 22 (16), 3255-3260 (2004).

21. Boellaard R, Standards for PET image acquisition and quantitative data analysis, J Nucl Med 50 Suppl 1, 11S-20S (2009).

22. Boellaard R, Oyen WJ, Hoekstra CJ, et al., The Netherlands protocol for standardisation and quantification of FDG whole body PET studies in multi-centre trials, Eur J Nucl Med Mol Imaging 35 (12), 2320-2333 (2008).

23. Petit SF, Aerts HJ, van Loon JG, et al., Metabolic control probability in tumour subvolumes or how to guide tumour dose redistribution in non-small cell lung cancer (NSCLC): an exploratory clinical study, Radiother Oncol 91 (3), 393-398 (2009).

24. Martel MK, Ten Haken RK, Hazuka MB, et al., Estimation of tumor control probability model parameters from 3-D dose distributions of non-small cell lung cancer patients, Lung Cancer 24 (1), 31-37 (1999).

25. Eriksen JG, Steiniche T and Overgaard J, The influence of epidermal growth factor receptor and tumor differentiation on the response to accelerated radiotherapy of squamous cell carcinomas of the head and neck in the randomized DAHANCA 6 and 7 study, Radiother Oncol 74 (2), 93-100 (2005).

26. Bonner JA, Harari PM, Giralt J, et al., Radiotherapy plus Cetuximab for Squamous-Cell Carcinoma of the Head and Neck, N Engl J Med 354 (6), 567-578 (2006).

27. Akimoto $T$, Hunter NR, Buchmiller $L$, et al., Inverse relationship between epidermal growth factor receptor expression and radiocurability of murine carcinomas, Clin Cancer Res 5 (10), 2884-2890 (1999).

28. Hebbar M, Wacrenier A, Desauw C, et al., Lack of usefulness of epidermal growth factor receptor expression determination for cetuximab therapy in patients with colorectal cancer, Anticancer Drugs 17 (7), 855-857 (2006).

29. Lenz HJ, Van Cutsem E, Khambata-Ford S, et al., Multicenter phase II and translational study of cetuximab in metastatic colorectal carcinoma refractory to irinotecan, oxaliplatin, and fluoropyrimidines, J Clin Oncol 24 (30), 4914-4921 (2006).

30. Chung KY, Shia J, Kemeny NE, et al., Cetuximab shows activity in colorectal cancer patients with tumors that do not express the epidermal growth factor receptor by immunohistochemistry, J Clin Oncol 23 (9), 1803-1810 (2005). 
31. Verel I, Visser GW, Boerman OC, et al., Long-lived positron emitters zirconium-89 and iodine-124 for scouting of therapeutic radioimmunoconjugates with PET, Cancer Biother Radiopharm 18 (4), 655-661 (2003).

32. Lu Y, Li X, Liang K, et al., Epidermal growth factor receptor (EGFR) ubiquitination as a mechanism of acquired resistance escaping treatment by the anti-EGFR monoclonal antibody cetuximab, Cancer Res 67 (17), 8240-8247 (2007).

33. van Baardwijk A, Bosmans G, Dekker A, et al., Time trends in the maximal uptake of FDG on PET scan during thoracic radiotherapy. A prospective study in locally advanced non-small cell lung cancer (NSCLC) patients, Radiother Oncol 82 (2), 145-152 (2007). 
Summary 


\section{SUMMARY}

In personalized medicine there is a growing interest to individualize treatment by the combination of systemic therapies and dose painting of radiation. It is expected that the implementation of these techniques will lead to improved local control of the tumour and consequently improved survival. In this thesis the usability of molecular imaging modalities for dose painting was assessed, i.e. part 1 describes the pre-treatment identification of areas with radioresistance to treatment, and part 2 describes the visualisation of drug uptake.

In Chapter 1, a general introduction is presented on the background of scientific research in the field of oncology, and the increasing need for personalize medicine in the future. Also the role of dose painting in personalize medicine is introduced, i.e. the concept of dose paining of radiation as a complementary approach to systemic therapies is described more in detail. More specifically, the rational behind the scientific research presented in this thesis is explained, why it is crucial to identify regions of biologic resistance (further described in part 1) and limited drug uptake (described in part 2). The non-invasive visualisation of drug uptake can be used to select beneficial drugs for patients and use the intra tumour distribution of drug uptake for dose painting, as regions with low (or limited) uptake can be compensated by higher radiotherapy doses.

\section{Part 1: Identification of radioresistance: Rational for dose painting}

In part 1 the identification of areas of biologic resistance before treatment is investigated. A tracer usable for dose painting is the glucose analogue 18F-deoxyglucose (FDG) for positron emission tomography (PET) imaging. In Chapter 2, it was investigated if the location of the residual metabolic-active areas after treatment could be identified before treatment starts. Overlap fractions (OF) were calculated between areas of residual metabolic activity with the pre-treatment FDG-uptake locations within the primary tumour. Visual interpretation in a dataset of patients treated at MAASTRO clinic showed that the pre-treatment high-FDG uptake areas largely corresponded with the metabolic active areas after treatment. Further quantification of these results showed that the residual areas largely corresponded with the pretreatment $50 \%$ SUV high uptake area (OF $=70 \%$ ). The average volume of this $50 \%$ SUV threshold was $39 \%$ of the original GTV volume. Also, the residual areas were located almost completely within the pre-treatment GTV (OF $=91 \%)$, indicating no geographic miss of radiotherapy. Validations of these results were performed in independent datasets from the Princess Margaret Hospital in Toronto (Chapter 3) and in a prospectively dataset from the Radboud University Medical Centre in Nijmegen (Chapter 4). The Nijmegen dataset yielded similar results, i.e. the residual areas corresponded 
largely with the $50 \%$ SUV threshold before treatment (OF $=68 \%$ ) and were located almost completely within the GTV (OF $=94 \%$ ). The volume of the pre-treatment $50 \%$ SUV threshold encompassed in this dataset $35 \%$ of pre-treatment GTV. The Toronto dataset yielded similar results with the pre-treatment $40 \%$ SUV threshold, i.e. the overlap of the residual areas was $71 \%$ with this threshold and encompassed $43 \%$ of the original GTV volume. That the $40 \%$ SUV threshold was used instead of the $50 \%$ threshold was due to differences of the absolute values and volumes of the thresholds between the Toronto dataset and the Dutch datasets.

Another important question to be answered before clinical trials can be launched is whether these high FDG uptake areas remain stable during a course of radiotherapy (Chapter 5). Therefore, the stability of the high FDG-uptake areas during radiotherapy was assessed in repeated FDG-PET-CT images before (day 0) and during treatment (day 7 and 14). Visual interpretation showed that the FDG uptake patrons remained stable during treatment. This was further quantified using an OF calculation, showing a large overlap (>70\%) for the 34-60\% SUV FDG high uptake areas on the day 0 scan with the scans on day 7 and 14 .

Therefore we concluded that FDG is an attractive tracer for dose painting, as FDG corresponds with several biologic characteristics important for radioresistance, due to the confirmed relationship with the location of residue, the stability of the uptake patterns during treatment, and the wide availability with standardized imaging protocols. Based on the results described in this thesis, we postulate that there is sufficient scientific basis to embark dose painting in carefully designed clinic trials, to test the assumption if FDG uptake reflects "treatment-resistance", potentially feasible using only a single FDG-PET scan before therapy.

\section{Part 2: Non invasive imaging of Drug uptake: From the bench to the clinic}

The second part of the thesis investigates the visualisation of the drug Cetuximab noninvasively. Cetuximab is a drug that targets the epidermal growth factor receptor (EGFR) that is related with tumour aggressiveness and overall treatment resistance. However, it is known that only a small sub-population of patients benefits from Cetuximab treatment, were often discrepancies between Cetuximab uptake and EGFR expression levels have been reported. Therefore, the non-invasive quantification of in vivo Cetuximab uptake tumours and healthy tissue could provide crucial prognostic information, i.e. selecting patients suitable for Cetuximab treatment and optimizing dosage, but also identifying intra tumour areas for dose painting.

To assess the binding of Cetuximab in vitro, an image probe using Cetuximab labelled with the fluorescent marker Oregon-Green 488 was successfully developed (Chapter 
6). Here, it was shown that binding of Cetuximab is strongly related with in vitro EGFR expression levels and that Cetuximab binds to the EGFR mutant EGFRvIll. Ex vivo immunohistochemistry analysis showed a very heterogeneous uptake of in vivo Cetuximab, but also a very heterogeneous EGFR expression throughout the tumour. Besides being heterogeneous, a disparity was reported were areas with low EGFR expression had a high Cetuximab uptake, and vice versa.

Contributing to this knowledge, another probe was developed to enable non-invasively imaging of Cetuximab uptake in pre-clinical and clinical situations. The results are described in Chapter 7. The long-lived positron emitter Zirconium-89 $\left({ }^{89} \mathrm{Zr}\right)$ for PET imaging was selected for the labelling with Cetuximab. As ${ }^{89} \mathrm{Zr}$ displays ideal characteristics for in vivo imaging, e.g. a long half life (78 hours) and the very high labelling efficiency, is was selected for imaging Cetuximab uptake. In vivo PET images in mice showed successful imaging that revealed a clear uptake of ${ }^{89} \mathrm{Zr}$-Cetuximab in EGFR positive tumours with high quality images (tumour-to-blood ratios from 3-5). However, again a large disparity was found between ${ }^{89} \mathrm{Zr}$-Cetuximab uptake and in vivo EGFR expression levels. The uptake in a high EGFR expressing cell line was two times lower than in intermediate EGFR expressing cell lines. These results were confirmed with ex vivo $\gamma$ counting biodistribution studies. Because of these successful pre-clinical results, a phase I imaging trial using ${ }^{89} \mathrm{Zr}$-Cetuximab was performed at MAASTRO clinic. This trial showed no additional toxicity associated with ${ }^{89} \mathrm{Zr}$-Cetuximab administration (Chapter 8). There was also selective targeting of ${ }^{89} \mathrm{Zr}$-Cetuximab in the tumour, although with very low tumour-to-blood ratios. This was expected as Cetuximab is only efficient in a small number of patients, and only six patients successfully finished the trial, and all patients had no EGFR mutation or overexpression.

Concluding, the disparity reported in these studies may help to explain the poor correlation often reported between Cetuximab efficacy and EGFR expression, as the results reveal additional mechanisms that can explain why EGFR expression levels alone may not be sufficient to explain therapy effects of Cetuximab in the clinic. 
Samenvatting 


\section{SAMENVATTING}

Er is een sterke behoefte om de behandeling verder te individualiseren door gebruik te maken van systemische behandelingen in combinatie met radiotherapie. Het wordt verwacht dat de implementatie van deze technieken zal leiden tot betere controle van de tumor en daardoor een beter overleving. In dit proefschrift worden moleculaire imaging technieken bekeken voor het sturen van de radiotherapie dosis, zogenaamd dose painting. In het eerste gedeelte van de thesis wordt onderzocht of imaging technieken voor behandeling de resistente zones kunnen aangeven voor behandeling. In het tweede gedeelte de visualisatie van medicijnen is geëvalueerd voor dose painting.

Hoofdstuk 1 beschrijft een generieke introductie over de achtergrond van het wetenschappelijk veld in de oncologie en de daaruit voortvloeiende noodzaak voor gepersonaliseerde behandelingen in de toekomst. Ook de rol van dose painting voor het individualiseren van de behandeling wordt geïntroduceerd, namelijk de rol van dose painting als een aanvullende techniek t.o.v. systemische behandelingen. Meer specifiek de achtergrond voor het wetenschappelijke werk van deze thesis is beschreven, waarom het belangrijk is om de resistente zones te identificeren (part 1 van de thesis), en waarom het belangrijk is om de opname van medicijnen te visualiseren (part 2 van de thesis).

\section{Part 1: Identificatie van radioresistentie voor dose painting}

De identificatie van de zones van biologische therapie resistentie door beeldvormende technieken is van belang voor dose painting. Een tracer geschikt voor deze taak, is de glucose variant ${ }^{18} \mathrm{~F}$-deoxyglucose (FDG) voor positron emission tomography (PET). In Hoofdstuk 2 is onderzocht of de locatie van overblijvende (residuele) metabolisch actieve zones kunnen worden geïdentificeerd voordat de behandeling start. Hiervoor zijn overlap fracties (OF) berekend tussen de residuele metabolisch actieve locaties met de primaire tumor. Visuele interpretatie van de resultaten, in een dataset van patiënten behandeld in MAASTRO Clinic, lieten zien dat hoge opname zones van FDG voor behandeling overeen komen met de metabolisch actieve locaties na behandeling. Verdere kwantificatie van deze resultaten lieten zien dat de residuele zones na behandeling grotendeels overeenkomen met de $50 \%$ SUV hoge opname zone (OF=70\%) voor behandeling. Het gemiddelde volume van deze $50 \%$ SUV threshold was $39 \%$ van het originele GTV volume. Ook bevonden deze residuele zones zich bijna volledig binnen het GTV volume voor behandeling (OF=91\%), wat laat zien dat er geen geografische misser is van de radiotherapie. Als validatie zijn studies gedaan in onafhankelijke dataset van het Princess Margaret Hospital (PMH) (Hoofdstuk 3) in Toronto en in een prospectieve dataset van de Radboud Universiteit in Nijmegen (Hoofdstuk 4). De dataset van Nijmegen liet vergelijkbare resultaten zijn, namelijk dat de residuele gebieden grotendeels 
overeen komen met de 50\% SUV hoge opname zone voor behandeling (OF=68\%). Ook bevonden deze residuele zones zich bijna volledig binnen het GTV volume voor behandeling (OF=94\%). Het volume van deze 50\% SUV threshold omvat gemiddeld genomen $35 \%$ van het totale GTV volume voor behandeling. Ook de Toronto dataset liet een vergelijkbaar resultaat zien met de $40 \%$ SUV threshold voor behandeling. De overlap van deze residuele zones was $71 \%$ met deze $40 \%$ SUV threshold en bevatte $43 \%$ van het originele GTV volume. Dat de $40 \%$ SUV threshold was gebruikt in tegenstelling tot de $50 \%$ threshold, kwam door verschillen in de absolute waardes van de thresholds gebruikt in de Totonto datasets en de Nederlandse datasets.

Een ander belangrijk vraagstuk voordat klinische studies gestart kunnen worden, is of deze hoge FDG opname zones stabiel blijven gedurende behandeling (Hoofdstuk 5). Daarom is de stabiliteit onderzocht van deze FDG opname thresholds gedurende behandeling in een herhaalde FDG-PET-CT dataset met images voor (dag 0) en gedurende behandeling (dag 7 en 14). Een visuele interpretatie van de beelden liet zien dat deze FDG opname patronen stabiel waren gedurende behandeling. Deze resultaten zijn verder gekwantificeerd een overlap fractie calculatie. De 34-60\% SUV opname thresholds lieten een hoge OF (>70\%) heeft tussen de scan op dag 0 en dag 7 en 14.

Concluderend, FDG is een attractieve tracer voor het gebruik van dose painting, omdat FDG gecorreleerd is met verschillende biologische kenmerken belangrijk voor resistentie, de bevestigde relatie met de locatie van het residu na behandeling, de stabiliteit gedurende behandeling, de goede beschikbaarheid en de gestandaardiseerde imaging protocollen. Gebaseerd op de resultaten beschreven in dit proefschrift, stellen we dat er voldoende wetenschappelijk bewijs is om te starten met dose painting in zorgvuldig opgezette klinische studies. Hier is de assumptie dat FDG opname resistentie aangeeft en dat deze resistentie kan worden geïdentificeerd met behulp van een enkele FDGPET scan voor behandeling.

\section{Part 2: Imaging van de opname medicijnen: Van de bench naar de kliniek}

Het tweede gedeelte van dit proefschrift onderzoekt de visualisatie van het medicijn Cetuximab. Cetuximab is een medicijn welke werkt tegen de epidermal growth factor receptor (EGFR), welke is gerelateerd met de agressiviteit van een tumor en algehele behandeling resistentie. Ondanks dat het veelvuldig wordt gebruikt in de kliniek, is het alleen werkzaam in een kleine subpopulatie van de patiënten, waar zelfs discrepanties tussen Cetuximab opname en EGFR expressie zijn vermeld. Daarom kan de kwantificatie van in vivo opname van Cetuximab in individuele tumoren belangrijke prognostische informatie bevatten. Hiermee kunnen patiënten die geschikt zijn voor een Cetuximab behandeling geselecteerd worden, alsook de optimale patiënt specifieke dosis 
bepaald worden. Ook kan deze 3-dimensionale Cetuximab opname gebruikt worden voor dose painting.

Om de in vitro binding van Cetuximab te onderzoeken is een imaging tracer ontwikkeld gebruikmakende van Cetuximab gelabelde met de fluorescente marker Oregon-Green 488 (Hoofdstuk 6). De resultaten lieten zien dat de binding van Cetuximab sterk gerelateerd is met de in vitro EGFR expressie. Ook is er een binding waargenomen van Cetuximab met de EGFR mutant EGFRvIII. Een ex vivo immuno-histochemie analyse liet een heterogene opname van in vivo Cetuximab zien, alsook een heterogene expressie van EGFR. Buiten deze heterogeniteit, is er zelfs een discrepantie gevonden tussen gebieden met lage EGFR expressie en hoge Cetuximab opname en visa versa.

Aanvullend hebben we een andere image tracer ontwikkeld voor de imaging van Cetuximab opname in pre-klinische en klinische situaties. De resultaten zijn beschreven in Hoofdstuk 7. Voor de labeling met Cetuximab is de positron emmiter Zirconium-89 $\left({ }^{89} \mathrm{Zr}\right)$ voor PET imaging geselecteerd. Dit omdat ${ }^{89} \mathrm{Zr}$ ideale karakteristieken heeft voor de in vivo beeldvorming, namelijk een lange halfwaarde tijd van (78 uur) en de hoge labeling effectiviteit met Cetuximab. Een studie met in vivo PET images was succesvol met duidelijke opname of ${ }^{89} \mathrm{Zr}$-Cetuximab in EGFR positieve tumors (tumor-to-blood ratios tussen de 3-5). Maar opnieuw was een discrepantie gevonden tussen ${ }^{89} \mathrm{Zr}$ Cetuximab opname en in vivo EGFR expressie. De opname in de hoge EGFR expressie cellijnen was twee keer lager dan de opname in de intermediaire EGFR expressie cellijnen. Deze resultaten zijn bevestigd met ex vivo gamma counting biodistributie studies. Gebaseerd op deze veelbelovende pre-klinische resultaten was een fase I klinische studie met ${ }^{89} \mathrm{Zr}$-Cetuximab gestart bij Maastro. Deze klinische studie liet zien dat de image tracer niet toxische was (Hoofdstuk 8). Er was selectieve opname van ${ }^{89} \mathrm{Zr}$ Cetuximab in de tumor, maar met lage tumor-to-blood ratios. Dit was verwacht omdat Cetuximab een therapeutische werking heeft in een kleine groep patiënten, en alleen zes patiënten geëvalueerd waren. Ook hadden deze patienten allemaal geen EGFR overexpressie in de tumour.

Concluderend, de discrepantie welke deze studies hebben laten zien, kan de slechte correlatie is tussen Cetuximab effectiviteit en EGFR expressie verklaren. De resultaten laten zien dat additionele mechanismen verantwoordelijk zijn voor Cetuximab opname in de kliniek. 
Dankwoord 


\section{DANKWOORD}

$\mathrm{Na}$ vier jaar hard werken is mijn proefschrift een feit. Ik heb de afgelopen jaren veel geleerd in de dynamische omgeving bij Maastro en hier is mijn interesse in de wetenschap pas echt tot stand gekomen. In dit dankwoord wil ik bij een aantal mensen in het bijzonder stilstaan die mij ondersteund hebben bij dit proefschrift:

Prof. Dr. Philippe Lambin: Promotor en directeur Maastro. Beste Philippe, dank dat je me het vertrouwen hebt gegeven om bij Maastro mijn promotieopdracht te vervullen. Je bent vanaf het eerste moment een geweldige inspiratie voor me geweest door je creativiteit in de wetenschap. Het is zeer bijzonder hoe je mensen kunt motiveren door je overweldigende enthousiasme. Ondanks je ontzettend drukke functie stond de deur altijd voor me open, zelfs als ik naar je toekwam met 'stomme' vragen. Je hebt me echt laten zien dat het denken in mogelijkheden veel beter werkt dan het denken in problemen. Daarbij kreeg ik echt het gevoel van "the sky is the limit". Bedankt voor alle mogelijkheden die je me hebt geboden!

Prof. Dr. Dirk De Ruysscher: Promotor. Beste Dirk, ook dankzij jou begeleiding is dit proefschrift tot stand gekomen. Je bent een geweldige bron van ideeën. Het is verbazingwekkend hoeveel kennis je hebt van (zowel binnen als buiten) het medisch domein en hoe je de translatie kan maken tussen vraagstukken in de fysica (of zelfs geschiedenis) en de vraagstukken waarmee wij geconfronteerd worden. Dank voor je grenzenloze enthousiasme, ik ken maar weinig mensen met zoveel passie voor de wetenschap. Dit resulteert dat je een inspiratie bent voor velen, alsook voor mij. Mede dankzij jou heb ik voor het wetenschappelijke pad gekozen.

Dr. Ir. Andre Dekker: Co-promotor. Beste Andre, bedankt voor je begeleidende rol in de beginjaren van mijn promotie. Je gaf me veel vrijheid, maar was altijd beschikbaar voor me als ik je wetenschappelijke oog nodig had. Zelfs na al die jaren, verbaas je me tegenwoordig nog steeds over het geweldige snelle inzicht dat je hebt. Je kan de lastigste vraagstukken doodsimpel laten lijken.

Prof. Dr. Bradly Wouters. Dear Brad, thank you for all the help with the lab-work of my thesis. You inspired me in all our talks about being a physicist in a biology environment. Thank you for making my visit to Canada possible, I enjoyed Toronto a lot.

Steven, paranimf. Bedankt voor alle mooie momenten tijdens onze reizen! Op een bepaalde manier blijven onze levens zich kruisen, eerst op de TU en later bij de UM. Ik ben blij dat we gedurende onze promoties elkaar zo goed hebben leren kennen en hoop je als vriend te mogen behouden. Veel succes met je opleiding tot klinisch fysicus en succes in het 'prachtige' Rotterdam. 
Geert: paranimf. Ondanks je immer aanwezige (Belgische) bescheidenheid, ben je iemand die veel in zijn mars heeft. Bedankt voor al die mooie verhalen die we gewisseld hebben de afgelopen jaren. Ik hoop dat ik je in de toekomst weer meer voor de wetenschap weet te prikkelen!

Ludwig: Bedankt voor al je hulp in het lab. Zonder die hulp was dit proefschrift nooit gelukt. Ik heb altijd veel waarde gehecht aan de kruisbestuiving van een bioloog en een fysicus. Al heb ik wel het idee dat je meer werk aan mij hebt gehad dan omgedraaid. Je hebt echt een ongekende werkmentaliteit, waarbij je, ondanks de gigantische hoeveelheid werk die je verzet, nooit de kwaliteit uit het oog verliest.

Mijn (ex)kamergenoten: Erik, Miguel, Wouter, Guillaume, Steven, Ruud, Marco, Esther, Enrica, Lucas, Davy en Tezontl, bedankt voor de gezellige tijd! Hopelijk kunnen we nog vaak tapas gaan eten en speciaal-biertjes gaan drinken!

Claudia: Je bent een hele grote hulp geweest bij het verzamelen van alle data. Zonder jouw inbreng waren de projecten nooit gelukt. Michel: bedankt voor je cruciale hulp bij alle PET vragen. Verder wil ik de klinisch fysici bedanken van onze afdeling: Geert, Lars, Bas, Peter, Brigitte, Erik, Michel, bedankt voor jullie hulp. Cary en Ruud, bedankt voor het aanvullen van mijn (beperkte) statistische kennis. Angela en Judith, bedankt voor jullie hulp bij al mijn stomme medische vragen.

Alle mensen van Maastro lab: Brad, Jan, Kasper, Barry, Natasja, Maud, Marianne, Roel, Nejla, Sherry, Younan, Ronald, Hilda, Kim, Kim, Tom, Michael en Guido. Bedankt dat jullie mij als 'die fysicus' thuis lieten voelen in het lab! Ik zal de teambuilding uitjes nooit vergeten. Vooral dat mooie moment toen ik als eerste over de streep ging bij het karten. Wel moet ik nog wat oefenen met aardappelen tussen mijn benen en Guitar Hero! Ook wil ik Twan en Chantal bedanken voor een geweldig leuke tijd in Toronto. I hope I can return the favour in Boston!

Ook buiten onze universiteit ben ik veel mensen dankbaar. Beste Guus en Lars, bedankt voor de labeling van het Zirconium in het VU medisch centrum. I also want to thank the people of the Princess Margaret Hospital in Toronto, especially Alexander Sun for making a validation of the work presented in this thesis possible. Ook ben ik de mensen van de Radboud Universiteit in Nijmegen zeer dankbaar voor hun hulp bij de prospectieve validatie: Johan Bussink, Wim Oyen, Annemieke Folgering. Ook wil ik Peter Vermaelen van de Universiteit Leuven bedanken voor de hulp bij de Zirconium projecten. Tilman Hackeng, altijd goed om een echte scheikundige bij onze projecten te betrekken. 
A special gratitude goes to Bob Gillies. Although our collaboration work was on the Radiomics projects and outside of this thesis, I want to thank you for the very nice time I had during my visits in Florida. You made me feel very welcome, and you and your group are an example for the scientific community.

Ook wil ik de studenten Marco, Ans, Roel, Emmanuel, Ralph en Katrin bedanken die ik met veel plezier heb begeleid tijdens hun stages en afstuderen van de TU. Ik hoop dat jullie net zoveel van mij geleerd hebben als ik van jullie. Also Emmanuel, Ralph and Katrin, thanks for strengthening the Radiomics team.

Verder zou ik ook de leden van de beoordelingscommissie willen bedanken. Prof. Dr. Wildberger, Prof. Dr. Gillies, Prof. Dr. Ir. Ter Haar Romeny en Prof. Dr. Verhaegen, bedankt voor het grondig bestuderen van mijn proefschrift en het voorzien van waardevolle opmerkingen. Verder wil ik ook de andere co-auteurs bedanken, welke ik nog niet genoemd heb.

Als laatste wil ik al mijn familie en vrienden bedanken. In het bijzonder mijn ouders. Lieve mama en papa: bedankt voor het aanhoren van al mijn verhalen de afgelopen jaren. Jullie hebben gezien hoe mijn leven in een stroomversnelling is gekomen na de start van mijn promotie. Daarbij hebben jullie me altijd ondersteund en van advies voorzien en zijn jullie altijd in mij blijven geloven. Mede door jullie hulp is dit proefschrift tot een succesvol einde gekomen. Pap, bedankt voor de hulp bij de kaft.

Tenslotte Desiree, mijn allerliefste. Bedankt voor al het geduld en vertrouwen dat je de afgelopen jaren in me hebt getoond. Al die dagen dat ik tot laat moest werken, of dat ik weer weg moest voor het werk. Je bent altijd begripvol geweest en hebt me altijd ondersteunt.

Laat nu ons avontuur samen beginnen, waar dan ook ter wereld!

Hugo 
Curriculum Vitae 


\section{CURRICULUM VITAE}

Hugo Aerts was born on July 31st 1979 in Valkenburg in the Netherlands. After high school at the Eurocollege in Maastricht, he studied Electrical Engineering and obtained his bachelor degree in 2002. Afterwards, he continued to study Electrical Engineering at the Eindhoven Institute of Technology (TU/e), with special interest in the fields of Biomedical Engineering and Artificial Intelligence. He combined these interests in his graduation work, consisting about pharmacokinetic modelling used in dynamic contrast enhanced MRI imaging, a joint project of the System Identification group of the TU/e and the Radiology Department at the Maastricht University. After completion of his graduation work, he obtained his Master of Science degree in 2006. Because he was very intrigued by the beauty of science, he continued his scientific education at Department of Radiation Oncology (MAASTRO) at Maastricht University as a PhD student under the supervision of Prof. Dr. Ph Lambin. During his PhD training he went to Canada in 2009 as a visiting scientist at the University of Toronto. He completed his $\mathrm{PhD}$ thesis in $\mathbf{2 0 1 0}$ in the field of image analysis and dose painting of radiation. In 2010 he was awarded a personal fellowship of the Dutch Cancer Society (KWF) for the duration of four years. With this grant he can continue his scientific interest and will work at the Harvard University in Boston, United States.

\section{Grants}

- Fellowship grant of the Dutch Cancer Society (KWF), 4 years of funding from 2010 to 2014. (Personal fellowship grant)

- Radiomics Grant, National Institute of Health (NIH). Main applicant: Moffitt Cancer Centre, 5 years of funding: 2010-2015 (co-applicant)

- Personal Travel Grant of the Dutch Cancer Society (KWF). Princess Margaret Hospital (PMH), University of Toronto, Canada, April-July 2009

- Personal Travel Grant Rene Vogels Stichting. Princess Margaret Hospital (PMH), University of Toronto, Canada April-July 2009

- Grant of Dutch Cancer Society (KWF), Non-invasive imaging of EGFR expression with Cetuximab-Zirconium, a new PET marker, 2008-2010 (co-applicant)

\section{Awards}

- ESTRO Travel Award 2006, Molecular Oncology for the Radiation Oncologist, Granada, Spain; ESTRO Travel Award 2007, Physics for Clinical Radiotherapy, Limassol, Cyprus; ESTRO Travel Award 2008, Imaging for Target Volume Determination, Prague, Czech Republic

- Alavi-Mandell award of the Society of Nuclear Medicine (SNM), SNM annual meeting in Salt Lake City, Utah, June 5-9 2010. 


\section{SCIENTIFIC PUBLICATIONS}

1. H.J.W.L. Aerts, L. Dubois, T.M. Hackeng, R. Straathof, R.K. Chiu, N.G. Lieuwes, B. Jutten, S.A. Weppler, G. Lammering, B.G. Wouters, P. Lambin, "Development and evaluation of a cetuximab-based imaging probe to target EGFR and EGFRvIII", Radiother Oncol. 2007 Jun;83(3):326-32, IF = 4.3

2. H.J.W.L. Aerts, G. Bosmans, A.A. van Baardwijk, A.L. Dekker, M. Öllers, P. Lambin, D. de Ruysscher, "Stability of 18F-Deoxyglucose uptake locations within the tumor during radiotherapy for non-small cell lung cancer: a prospective study", Int J Radiat Oncol Biol Phys. 2008 Aug 1;71(5):1402-7, IF = 4.6

3. H.J.W.L. Aerts, N.A.W. van Riel, W.H. Backes, "System identification theory in pharmacokinetic modeling of dynamic contrast enhanced magnetic resonance imaging: influence of contrast injection", Magn Reson Med. 2008 May;59(5):1111-9, IF = 3.2

4. G. Janssens, J. Orban de Xivry, H.J.W.L. Aerts, G. Bosmans, A.L.A.J. Dekker, B. Macq, "Improving physical behavior in image registration", ICIP 2008, 15th IEEE International Conference on Image Processing, 12-15 October 2008, 2952-2955

5. H.J.W.L. Aerts, L. Dubois, L. Perk, P. Vermaelen, G.A.M.S. van Dongen, B.G. Wouters, P. Lambin, "Disparity between in vivo Zirconium-89-labeled Cetuximab uptake and EGFR expression", J Nucl Med. 2009 Jan;50(1):123-31, IF = 6.4

6. M.H.M. Janssen, H.J.W.L. Aerts, M.C. Öllers, G. Bosmans, J.A. Lee, J. Buijsen, D. De Ruysscher, P. Lambin, G. Lammering, A.L.A.J. Dekker, "Tumor delineation based on time-activity curve differences assessed with dynamic fluorodeoxyglucose positron emission tomography-computed tomography in rectal cancer patients", Int J Radiat Oncol Biol Phys. 2009 Feb $1 ; 73(2): 456-65, \mathrm{IF}=4.6$

7. H.J.W.L. Aerts, A.A. van Baardwijk, S.F. Petit, C. Offermann, J.V. van Loon, R. Houben, A.M. Dingemans, R. Wanders, L. Boersma, J. Borger, G. Bootsma, W. Geraedts, C. Pitz, J. Simons, B.G. Wouters, M. Oellers, P. Lambin, G. Bosmans, A.L. Dekker, D. De Ruysscher, "Identification of residual metabolic-active areas within individual NSCLC tumours using a preradiotherapy (18)Fluorodeoxyglucose-PET-CT scan", Radiother Oncol. 2009 Jun;91(3):386$92, \mathrm{IF}=4.3$

8. S.F. Petit, H.J.W.L. Aerts, J.G. van Loon, C. Offermann, R. Houben, B. Winkens, M.C. Ollers, P. Lambin, D. De Ruysscher, A.L. Dekker, "Metabolic control probability in tumour subvolumes or how to guide tumour dose redistribution in non-small cell lung cancer (NSCLC): an exploratory clinical study", Radiother Oncol. 2009 Jun;91(3):393-8, IF = 4.3 
9. D. De Ruysscher, A. Houben, H.J.W.L. Aerts, C. Dehing, R. Wanders, M. Ollers, A.M. Dingemans, M. Hochstenbag, L. Boersma, J. Borger, A. Dekker, P. Lambin, "Increased (18)Fdeoxyglucose uptake in the lung during the first weeks of radiotherapy is correlated with subsequent Radiation-Induced Lung Toxicity (RILT): A prospective pilot study", Radiother Oncol. 2009 Jun;91(3):415-20, IF = 4.3

10. B. Jutten, L. Dubois, Y. Li, H.J.W.L. Aerts, B.G. Wouters, P. Lambin, J. Theys, G. Lammering, "Binding of cetuximab to the EGFRvIII deletion mutant and its biological consequences in malignant glioma cells", Radiother Oncol. 2009 Sep;92(3):393-8, IF = 4.3

11. K. Jaspers, H.J.W.L. Aerts, T. Leiner, N.A. van Riel, M.J. Post, and W.H. Backes, "Reliability of pharmacokinetic parameters: small vs. medium-sized contrast agents", Magn Reson Med. 2009 Sep;62(3):779-87, IF = 3.2

12. M.H.M. Janssen, M.C. Öllers, R.G. Riedl, J. van den Bogaard, J. Buijsen, H.J.W.L. Aerts, P. Lambin, Guido Lammering, "Accurate Prediction of Pathological Rectal Tumor Response after Two Weeks of Preoperative Radiochemotherapy Using (18)F-FluorodeoxyglucosePositron Emission Tomography-Computed Tomography Imaging", Int J Radiat Oncol Biol Phys. 2010 Jun 1;77(2):392-9, IF = 4.6

13. R.J. Kierkels, W.H. Backes, M. Janssen, J. Buijsen, R.G. Beets-Tan, P. Lambin, G. Lammering, M. Oellers, H.J.W.L. Aerts, "Comparison between Perfusion-CT and Dynamic ContrastEnhanced MRI in Rectal Cancer", Int J Radiat Oncol Biol Phys. 2010 Jun 1;77(2):400-8, IF = 4.6

14. M.H.M. Janssen, H.J.W.L. Aerts, R.G.J. Kierkels, W.H. Backes, M.C. Öllers, J. Buijsen, P. Lambin, G. Lammering, "Tumor perfusion increases during hypofractionated short-course radiotherapy in rectal cancer: sequential perfusion-CT findings", Radiother Oncol. 2010 Feb;94(2):156-60, IF = 4.3

15. J. van Loon, M.H.M. Janssen, D. De Ruysscher, M. Oellers, H.J.W.L. Aerts, L. Dubois, M. Hochstenbag, A.C. Dingemans, R. Lalisang, B. Brans, A.D. Windhorst, G.A. van Dongen, H. Kolb, J. Zhang, P. Lambin, "PET imaging of hypoxia with the new tracer 18F-HX4: A phase I trial", Eur J Nucl Med Mol Imaging. 2010 Aug;37(9):1663-8, IF = 4.5

16. H.J.W.L. Aerts, P. Lambin, D. De Ruysscher, "Editorial: FDG for dose painting: A rational choice", in press Radiother Oncol, IF $=4.3$

17. E. Rios-Velazquez, H.J.W.L. Aerts, C. Oberije, D. De Ruysscher, P. Lambin, "Prediction of residual metabolic activity after treatment in NSCLC patients", Acta Oncol. 2010 Oct;49(7):1033-9., IF = 2.3 
18. C. Oberije, H.J.W.L. Aerts, S. Yu, D. De Ruysscher, P. Menheere, M. Hilvo, H. van der Weide, B. Rao, P. Lambin, "Development and validation of a prognostic model using blood biomarker information for prediction of survival of non-small cell lung cancer patients treated with combined chemotherapy and radiation or radiotherapy alone (NCT00181519, NCT00573040, NCTO0572325)", in press Int J Radiat Oncol Biol Phys, IF = 4.6

19. P. Lambin P, S.F. Petit, H.J.W.L. Aerts, W.J. van Elmpt, C.J. Oberije, M.H. Starmans, R.G. van Stiphout, G.A. van Dongen, K. Muylle, P. Flamen, A.L. Dekker, D. De Ruysscher, "The ESTRO Breur Lecture 2009. From population to voxel-based radiotherapy: exploiting intra-tumour and intra-organ heterogeneity for advanced treatment of non-small cell lung cancer", Radiother Oncol. 2010 Aug;96(2):145-52, IF = 4.3

20. M.F. Jimenez, A. van Baardwijk, H.J.W.L. Aerts, D. De Ruysscher, N.M. Novoa, G. Varela, P. Lambin, "Effectiveness of surgery and individualized high-dose hyperfractionated accelerated radiotherapy on survival in clinical stage I non-small cell lung cancer. A propensity score matched analysis", in press Radiother Oncol, IF $=4.3$

21 A.J. Buckler, H.J.W.L. Aerts, B. Bendriem, C. Bendtsen, R. Boellaard, J.M. Boone, L. Bresolin, D. Burstein, P.E. Cole, J.J. Conklin, G.S. Dorfman, P.S. Douglas, R. Dunnick, W. Eidsaunet, C. Elsinger, R.A. Frank, C. Gatsonis, M.L. Giger, D. Gustafson, S.N. Gupta, O.S. Hoekstra, E.F. Jackson, L. Karam, G.J. Kelloff, P.E. Kinahan, G. McLennan, C.G. Miller, P.D. Mozley, K.E. Muller, K. O'Donnell, R. Patt, D. Raunig, M. Rosen, H. Rupani, L.H. Schwartz, B.A. Siegel, A.G. Sorensen, R.L. Wahl, J.C. Waterton, W. Wolf, G. Zahlmann, B. Zimmerman, D.C. Sullivan, "A Collaborative Enterprise for Multi-Stakeholder Participation in the Advancement of Quantitative Imaging", accepted Radiology, IF = 6.3

22 A.J. Buckler, H.J.W.L. Aerts, B. Bendriem, C. Bendtsen, R. Boellaard, J.M. Boone, L. Bresolin, D. Burstein, P.E. Cole, J.J. Conklin, G.S. Dorfman, P.S. Douglas, R. Dunnick, W. Eidsaunet, C. Elsinger, R.A. Frank, C. Gatsonis, M.L. Giger, D. Gustafson, S.N. Gupta, O.S. Hoekstra, E.F. Jackson, L. Karam, G.J. Kelloff, P.E. Kinahan, G. McLennan, C.G. Miller, P.D. Mozley, K.E. Muller, K. O'Donnell, R. Patt, D. Raunig, M. Rosen, H. Rupani, L.H. Schwartz, B.A. Siegel, A.G. Sorensen, R.L. Wahl, J.C. Waterton, W. Wolf, G. Zahlmann, B. Zimmerman, D.C. Sullivan, "Quantitative Imaging Test Approval and Biomarker Qualification: Inter-related but Distinct Activities", accepted Radiology, IF $=6.3$

23 M.H.M. Janssen, H.J.W.L. Aerts, J. Buijsen, P. Lambin, G. Lammering, M.C. Öllers, "FDG uptake corresponds with tumor perfusion as assessed with sequential combined PET-CT and perfusion-CT imaging in rectal cancer", accepted Int J Radiat Oncol Biol Phys., IF = 4.6 